\title{
Universal Coefficient Theorems in Equivariant KK-theory
}

\author{
Dissertation \\ zur Erlangung des mathematisch-naturwissenschaftlichen \\ Doktorgrades \\ "Doctor rerum naturalium" \\ der Georg-August-Universität Göttingen \\ vorgelegt
}

von

Manuel Köhler

aus Marburg

Göttingen 2010 
1. Gutachter: Ralf Meyer

2. Gutachter: Thomas Schick

Tag der mündlichen Prüfung: 


\section{Contents}

$\begin{array}{ll}\text { Acknowledgements } & 3\end{array}$

Introduction $\mathbf{5}$

1 The Action of a Cyclic Group of Prime Order . . . . . . . . . . . . . . . 6

2 The Action of a Finite Cyclic Group . . . . . . . . . . . . . . . 7

$3 \quad C^{*}$-algebras over Finite Topological Spaces . . . . . . . . . . . . . 8

4 Homological Algebra in Triangulated Categories . . . . . . . . . . . . . 9

$\begin{array}{ll}\text { Background } & \mathbf{1 1}\end{array}$

5 Triangulated Categories and the Construction of Invariants . . . . . . . . 11

6 The Bootstrap Class . . . . . . . . . . . . . . . . . . . . . . . 14

7 A General UCT Criterion . . . . . . . . . . . . . . . . . . . 16

Equivariant KK-theory and the UCT for the Action of a Cyclic Group of $\begin{array}{lr}\text { Prime Order } & 17\end{array}$

8 Equivariant KK-theory as a Triangulated Category . . . . . . . . . . . . 17

8.1 The Categories $\mathfrak{K}_{\mathfrak{K}}^{G}$ and $\mathfrak{K}_{\mathfrak{K}_{*}}^{G} \ldots \ldots \ldots \ldots \ldots$

8.2 Properties of the Hom-like Invariants in the $G$-equivariant Case . . 18

9 A close Look at the Bootstrap Class . . . . . . . . . . . . . . . . . 20

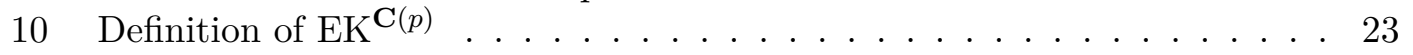

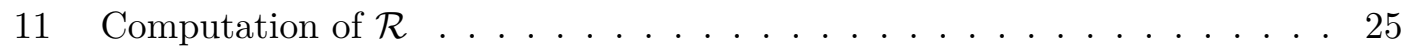

11.1 Preparation . . . . . . . . . . . . . . . . 25

11.2 Baaj-Skandalis Duality . . . . . . . . . . . . . 26

11.3 The Green-Julg Theorem . . . . . . . . . . . . . . . . . 28

$11.4 \quad$ A Subring of $\mathcal{R} \ldots \ldots \ldots \ldots \ldots \ldots$

11.5 The First Exact Triangle . . . . . . . . . . . . . . . . . 30

11.6 The Second Exact Triangle . . . . . . . . . . . . . . . . 30

11.7 Morphisms from and into $C_{u} \ldots \ldots \ldots \ldots$. . . . . . . 32

11.8 The Subring $\mathrm{KK}_{0}^{\mathbf{C}(p)}\left(C_{u}, C_{u}\right) \ldots \ldots \ldots \ldots \ldots$

11.9 Summary . . . . . . . . . . . . . . . . 37

12 Projective Resolutions of $\mathcal{R}$-Modules . . . . . . . . . . . . . . . 38

12.1 Conventions and Generalities on Rings and Modules . . . . . . . . 40

12.2 Preliminaries on Dedekind Domains . . . . . . . . . . . . . . 41

$12.3 \quad$ Lattices over $\mathbb{Z} \mathbf{C}(p) \ldots \ldots \ldots$

12.4 Lattices over ${ }_{2} \mathcal{R}_{2} \ldots \ldots \ldots \ldots \ldots \ldots$

12.5 Lattices over $\mathcal{R}$ and the Proof of Theorem $12.1 \ldots \ldots$. . . . . 52 
A UCT for Actions of Finite Cyclic Groups $\quad 57$

13 Introduction . . . . . . . . . . . . . . . . . . . . 57

14 The Framework . . . . . . . . . . . . . . . . . . . 58

14.1 The Invariant . . . . . . . . . . . . . . . . . . . 58

14.2 The Bootstrap Class . . . . . . . . . . . . . . . . 59

15 Basic Calculations in $\mathfrak{C}^{G} \ldots \ldots \ldots \ldots \ldots$. . . . . . . . . . 60

15.1 Duality . . . . . . . . . . . . . . . . . 60

15.2 Generators of $\mathfrak{C}^{G} \ldots \ldots \ldots \ldots 6 . \ldots \ldots 6$

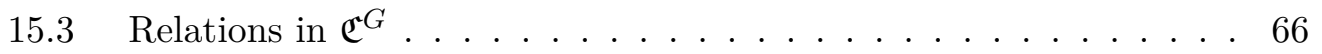

16 Projective Resolutions of Length $1 \ldots \ldots$. . . . . . . . . . . . 68

16.1 Split Categories and Tensor products . . . . . . . . . . . 69

$16.2 \quad$ A Special Case . . . . . . . . . . . . . . . . . . 72

16.3 The General Case . . . . . . . . . . . . . . . . 78

Universal Coefficient Theorems for $C^{*}$-algebras over Topological Spaces 81

$17 C^{*}$-algebras over Finite Topological Spaces . . . . . . . . . . . . . . . . . . 81

17.1 Basic Notions . . . . . . . . . . . . . . . . . 81

17.2 Functoriality . . . . . . . . . . . . . . . . 82

17.3 Specialization Order . . . . . . . . . . . . . . . 82

17.4 Representation of Finite Topological Spaces as Directed Graphs . . 83

$18 \mathfrak{K} \mathfrak{K}(X)$ and Filtrated K-theory . . . . . . . . . . . . . . . . . 84

$18.1 X$-equivariant KK-theory $\ldots \ldots \ldots \ldots \ldots$

18.2 Filtrated K-theory . . . . . . . . . . . . . . . 85

18.3 The Representability Theorem . . . . . . . . . . . . . . 86

18.4 Functoriality . . . . . . . . . . . . . . 86

19 The Bootstrap Class and the UCT Criterion . . . . . . . . . . . 88

19.1 Comparing Bootstrap Classes . . . . . . . . . . . . . . 88

19.2 The UCT Criterion and Embedding Results . . . . . . . . . . . . 88

20 Positive Results and Counterexamples . . . . . . . . . . . . . . . . . . . 90

21 The Complete Description . . . . . . . . . . . . . . . . . . . . . . . . . . 91

$\begin{array}{ll}\text { Appendix } & 95\end{array}$

22 Facts about Cyclotomic Polynomials . . . . . . . . . . . . . 95

23 Miscellaneous Results . . . . . . . . . . . . . . . . . . . . 100 


\section{Acknowledgements}

There are a number of people without whom this thesis might not have been written, and to whom I am greatly indebted.

To Sofia, who accepted my occasional absentmindedness, supported me when I reached a dead end and gave me the freedom I needed when I found a way out. To my parents, whose faith in me has always been a source of encouragement and inspiration throughout my life. A very special thank you for providing a "writing space" and for nurturing me through the final weeks of writing. To my friends who provided me with hours of delightful distractions and made sure that I would not get lost in the world of homological algebra and KK-theory.

To my advisor Ralf Meyer, who always had time to answer my questions and from whose farsightedness in discussing interesting research problems I benefited a lot. To Rasmus Bentmann with whom I really enjoyed working. A special thank you to Rasmus and Oliver Fritzen for reading the final version of the thesis. To Lukasz Grabowski, with whom I spend afternoons to discuss my results and whose dedication to mathematics has been a valuable source of motivation to me. To everyone in and around our work group, who actively participated in the many informal student seminars and thereby contributed to the great atmosphere in Göttingen that I enjoyed during the last two years. 



\section{Introduction}

Non-commutative topology studies $C^{*}$-algebras by regarding them as generalizations of topological spaces. This often means trying to use ideas from algebraic topology, in particular, by constructing invariants, which associate simpler objects such as abelian groups, rings or modules with a given $C^{*}$-algebra. Probably the most prominent example of a generalization of a topological invariant is $\mathrm{K}$-theory [44]. It associates a $\mathbb{Z} / 2$-graded abelian group $\mathrm{K}_{*}(A)$ with a $C^{*}$-algebra $A$.

A great advancement in non-commutative topology was the classification of $A F$ algebras via K-theory by Elliott in 1976 [14]. Today, K-theory is still one of the most important tools for the classification of $C^{*}$-algebras. Another subject was the study of extensions of $C^{*}$-algebras, which led to the development of the Ext-functor, which is known to agree with K-homology in the commutative (unital) case. Both K-theory and its dual, K-homology, are closely connected to index theory of elliptic pseudo-differential operators.

A big step up the ladder was Kasparov's construction of bivariant K-theory in 1980 [22], which contains both K-theory and K-homology as special cases. It associates a $\mathbb{Z} / 2$ graded abelian group $\mathrm{KK}_{*}(A, B)$ with separable $C^{*}$-algebras $A$ and $B$. A remarkable feature of KK-theory is that it comes with the so called Kasparov product

$$
\mathrm{KK}_{*}(A, B) \otimes \mathrm{KK}_{*}(B, C) \rightarrow \mathrm{KK}_{*}(A, C) .
$$

Besides its importance as a very flexible and conceptually satisfactory invariant of $C^{*}$ algebras, KK-theory also yields results outside pure non-commutative topology. The most prominent example is probably the verification of the Novikov conjecture by applications of KK-theory to the Baum-Connes conjecture for a large class of groups ([2] and $[17])$.

K- and KK-theory are related by the Universal Coefficient Theorem (UCT) of Rosenberg and Schochet [41], which states that for separable $C^{*}$-algebras $A$ and $B$ with $A$ belonging to a certain bootstrap class, there is a short exact sequence

$$
\operatorname{Ext}^{1}\left(\mathrm{~K}_{*}(S A), \mathrm{K}_{*}(B)\right) \longmapsto \mathrm{KK}_{*}(A, B) \rightarrow \operatorname{Hom}\left(K_{*}(A), K_{*}(B)\right) .
$$

Here $S B:=\mathcal{C}_{0}(\mathbb{R}, B)$ denotes the suspension of $B$. This short exact sequence splits in a non-natural manner. Since K-theory, due to its more explicit definition in terms of projections and unitaries, is easier to calculate than KK-theory, the UCT proves to be very useful in order to calculate the KK-groups. Apart from that, it plays an important role in the classification of $C^{*}$-algebras by $\mathrm{K}$-theoretic invariants: The corresponding sequence for $A=B$ is an extension of rings with the product in $\operatorname{Ext}^{1}\left(\mathrm{~K}_{*}(A), \mathrm{K}_{*}(A)\right)$ being zero, therefore, $\operatorname{KK}_{*}(A, A)$ is a nilpotent extension of $\operatorname{Hom}\left(\mathrm{K}_{*}(A), \mathrm{K}_{*}(A)\right)$-this 


\section{Introduction}

shows that isomorphisms in K-theory lift to isomorphisms in KK-theory. Further results by Kirchberg and Phillips then show that every KK-equivalence between $A$ and $B$, which sends $\left[1_{A}\right]$ in $\mathrm{K}_{0}(A)$ to $\left[1_{B}\right] \in \mathrm{K}_{0}(B)$, actually comes from an actual isomorphism of $C^{*}$-algebras, provided that $A$ and $B$ are unital, nuclear, separable, purely infinite, in the bootstrap class and simple [34]. Both facts together give the following strong classification result: $C^{*}$-algebras $A$ with the above mentioned properties are completely classified by $\left(\mathrm{K}_{*}(A),\left[1_{A}\right]\right)$.

$C^{*}$-algebras may carry additional structures such as a group action, which encodes a certain symmetry or the structure of a $C^{*}$-algebra over a space, which encodes a given ideal structure. In these cases there are so called equivariant versions of KKtheory, generalizations of usual KK-theory, which capture these additional pieces of information. It is very interesting to ask for a UCT in these generalized contexts: It serves as a valuable tool for computing equivariant KK-groups. Moreover, a UCT in a generalized context is closely connected to further classification results. The task is to find an invariant $\mathrm{F}$, which takes values in an abelian category and is relatively easy to understand, and then to derive a short exact sequence, which computes equivariant KK-theory in terms of F.

Take for instance the classification result of Kirchberg and Phillips: Trying to remove the condition of $A$ being simple means having to deal with a $C^{*}$-algebra $A$ over its primitive ideal space $\operatorname{Prim}(A)$. Similarly, one way to classify group actions on $C^{*}$ algebras would be to derive a universal coefficient theorem for equivariant KK-theory and then to prove a theorem similar to the result of Kirchberg and Phillips, which allows for lifting equivariant KK-equivalences to equivariant isomorphisms of $C^{*}$-algebras. The aim of this thesis is to examine in which contexts a universal coefficient theorem can be derived.

There are three main results, which we will explain in more detail in the following.

\section{The Action of a Cyclic Group of Prime Order}

We will prove an equivariant Universal Coefficient Theorem for $C^{*}$-algebras equipped with an action of a cyclic group of prime order $p$, in the following denoted by $\mathbf{C}(p)$. A naïve generalization of the usual UCT, where one replaces K- and KK-theory by their $\mathbf{C}(p)$-equivariant versions, has no chance to hold. This is due to the fact that $\mathbf{C}(p)$ has infinite homological dimension. In [40], an article concerning $C^{*}$-algebras with an action of a compact Lie group $G$ satisfying the Hodgkin condition, Rosenberg and Schochet obtain merely a spectral sequence which relates $\mathrm{KK}^{G}$ and $\mathrm{K}^{G}$. This relation is not as strong as in the non-equivariant case, for example isomorphisms in $\mathrm{K}^{G}$ can in general not be lifted to $\mathrm{KK}^{G}$-equivalences.

Therefore, we choose a different approach and replace $\mathbf{C}(p)$-equivariant K-theory by an invariant, which carries more information. Let us denote this invariant by $\mathrm{EK}^{\mathbf{C}(p)}$ for extended K-theory. It consists of the usual K-theory, the K-theory of the crossed product and $\mathrm{KK}^{\mathrm{C}(p)}\left(C_{u}, \ldots\right)$ - here, $C_{u}$ is the mapping cone of the unital embedding of $\mathbb{C}$ into $\mathcal{C}(\mathbf{C}(p))$. It has a canonical module structure over a ring $\mathcal{R}$, which consists of all 
$\mathrm{KK}^{\mathbf{C}(p)}$-morphisms between $\mathbb{C}, \mathcal{C}(\mathbf{C}(p))$ and $C_{u}$. We also define a bootstrap class $\mathfrak{B} \mathbf{C}(p)$ analogous to the bootstrap class of Rosenberg and Schochet in [40]. The main theorem reads as follows:

Theorem 1.1. Let $A$ be a separable $\mathbf{C}(p)$ - $C^{*}$-algebra in $\mathfrak{B}^{\mathbf{C}(p)}$. Then for every separable $\mathbf{C}(p)-C^{*}$-algebra $B$, there is a natural short exact sequence

$$
\operatorname{Ext}_{\mathcal{R}}^{1}\left(\operatorname{EK}^{\mathbf{C}(p)}(S A), \operatorname{EK}^{\mathbf{C}(p)}(B)\right) \longmapsto \operatorname{KK}_{*}^{\mathbf{C}(p)}(A, B) \rightarrow \operatorname{Hom}_{\mathcal{R}}\left(\operatorname{EK}^{\mathbf{C}(p)}(A), \operatorname{EK}^{\mathbf{C}(p)}(B)\right) .
$$

We will prove the theorem by using a certain exactness property of $\mathcal{R}$-modules of the form $\operatorname{EK}^{\mathbf{C}(p)}(A)$ to show that these modules have a projective resolution of length 1 . At a crucial point, a classification result for lattices over the integral group ring of $\mathbf{C}(p)$ will be used in order to understand modules over the more complicated ring $\mathcal{R}$. To the author's knowledge, there are no classification results of this kind for, say, general finite groups. This seems to be the main obstacle to derive a UCT short exact sequence for more general groups.

\section{The Action of a Finite Cyclic Group}

We will prove a Universal Coefficient Theorem for $C^{*}$-algebras equipped with an action of a finite cyclic group $G$, which allows us to compute equivariant KK-groups in terms of a K-theoretic invariant after inverting the group order. Let $G$ be a finite cyclic group of order o. We will define an invariant $\mathrm{LK}^{G}$ taking values in an abelian category. Furthermore we will define a bootstrap class $\mathfrak{B}^{G}\left[\mathbf{o}^{-1}\right]$ as a subcategory of all separable $G$ - $C^{*}$-algebras. The main theorem reads as follows

Theorem 2.1. Let $G$ be a finite cyclic group of order $\mathbf{o}$ and $A$ and $B$ separable $G-C^{*}$ algebras with $A$ in $\mathfrak{B}^{G}\left[\mathbf{o}^{-1}\right]$. Then there is a natural short exact sequence

$$
\operatorname{Ext}_{\mathfrak{A}}^{1}\left(\operatorname{LK}^{G}(S A), \operatorname{LK}^{G}(B)\right) \longmapsto \operatorname{KK}_{*}^{G}(A, B)\left[\mathbf{o}^{-1}\right] \rightarrow \operatorname{Hom}_{\mathfrak{A}}\left(\operatorname{LK}^{G}(A), \operatorname{LK}^{G}(B)\right) .
$$

Hom and Ext are taken in the abelian category $\mathfrak{A}=\mathfrak{M o d}\left(\mathfrak{C}^{G}\left[\mathbf{o}^{-1}\right]\right)_{\mathrm{c}}^{\mathbb{Z} / 2}$.

Here, $\mathfrak{C}^{G}\left[\mathbf{o}^{-1}\right]$ denotes $\mathfrak{K} \mathfrak{K}^{G}\left[\mathbf{o}^{-1}\right]$ restricted to $\left\{\mathcal{C}(G)^{H} \mid H \leq G\right\}$ and $\mathfrak{M o d}\left(\mathfrak{C}^{G}\left[\mathbf{o}^{-1}\right]\right)_{\mathrm{c}}^{\mathbb{Z} / 2}$ is the category of countable $\mathbb{Z} / 2$-graded modules over $\mathfrak{C}^{G}\left[\mathbf{o}^{-1}\right]$. The reason for inverting the group order is mainly of technical nature: As mentioned before, the nonexistence of classification results for lattices over the rings $\mathbb{Z} G$ and $\operatorname{Rep}(G)$ for a general finite group $G$ appears to be the main obstacle to a UCT of the form

$$
\operatorname{Ext}_{\mathfrak{A}}^{1}(F(S A), F(B)) \longmapsto \operatorname{KK}_{*}^{G}(A, B) \rightarrow \operatorname{Hom}_{\mathfrak{A}}(F(A), F(B))
$$

for some homological invariant $F$ taking values in an abelian category $\mathfrak{A}$. We can overcome this difficulty by inverting the group order $\mathbf{o}$ since $\mathbb{Z}\left[\mathbf{o}^{-1}\right] G$ is isomorphic to a direct sum of Dedekind domains of the form $\mathbb{Z}\left[\mathbf{o}^{-1}, \theta_{m}\right]$ for $m \mid \mathbf{o}$ and $\theta_{m}$ a primitive $m$-th root of unity (see Theorem 22.8 in the appendix). Hence modules over $\mathbb{Z}\left[\mathbf{o}^{-1}\right] G$ are easier to understand. Although the result is meant to generalize the UCT for actions of a cyclic 


\section{Introduction}

group of prime order (up to the inversion of the group order), the means by which we prove it are quite different. No exactness properties of modules over $\mathfrak{C}^{G}\left[\mathbf{o}^{-1}\right]$ will be used, we will rather show that the category of modules over $\mathfrak{C}^{G}\left[\mathbf{o}^{-1}\right]$ is equivalent to the category of modules over a certain direct sum of Dedekind domains. This will show that every module over $\mathfrak{C}^{G}\left[\mathbf{o}^{-1}\right]$ has a projective resolution of length 1.

\section{$3 C^{*}$-algebras over Finite Topological Spaces}

As indicated above, in order to proceed in the $C^{*}$-algebra classification programme, one is strongly interested in removing the simplicity condition in the classification result of Kirchberg and Phillips. The aim here is to compute $\mathrm{KK}_{*}(X ; A, B)$ by a Universal Coefficient Theorem for a topological space $X$ and $C^{*}$-algebras $A$ and $B$ over $X$ for $A$ being in a certain bootstrap class $\mathcal{B}(X)$. In [28], Ralf Meyer and Ryszard Nest tried to derive a UCT short exact sequence, which computes $\operatorname{KK}(X ; A, B)$ for a finite $T_{0}$ space $X$ by filtrated K-theory (in the following denoted by FK). They derive the desired short exact sequence in the case of the totally ordered space $O_{n}$ with $n$ points, i.e.

$$
O_{n}=\{1,2, \ldots, n\}, \quad \tau_{O_{n}}=\{\{1\},\{1,2\}, \ldots, X\} .
$$

A $C^{*}$-algebra $A$ over this space is essentially the same as a $C^{*}$-algebra $A$ together with a finite increasing chain of ideals

$$
\{0\}=I_{0} \triangleleft I_{1} \triangleleft I_{2} \triangleleft I_{3} \triangleleft \cdots \triangleleft I_{n-1} \triangleleft I_{n}=A .
$$

On the other hand they give an example of a finite $T_{0}$ space $Y$, for which the following strong non-UCT statement holds: There are $A$ and $B$ in $\mathcal{B}(Y)$ with isomorphic filtrated $\mathrm{K}$-theory which are not $\mathrm{KK}_{Y}$ equivalent.

In his diploma thesis [4], Rasmus Bentmann showed that there is a more general type of spaces, for which there is a UCT short exact sequence, which computes $\mathrm{KK}(X ; A, B)$ by filtrated K-theory. Let us say that these spaces are of type $A$ (for a definition see 20.1).

We will complete the picture by showing the converse, i.e. that spaces of type $A$ are indeed the most general type of spaces, for which there is a UCT short exact sequence, which computes $\operatorname{KK}(X ; A, B)$ by filtrated $\mathrm{K}$-theory. The resulting theorem reads as follows:

Theorem 3.1. Let $X$ be a finite $T_{0}$ space. The following statements are equivalent:

(1) Let $A \in \mathcal{B}(X)$ and $B$ be a separable $C^{*}$-algebra over $X$. Then there is a short exact UCT sequence

$$
\operatorname{Ext}_{\mathcal{N} \mathcal{T}_{X}}(\mathrm{FK}(S A), \mathrm{FK}(B)) \longmapsto \mathrm{KK}_{*}(X ; A, B) \rightarrow \operatorname{Hom}_{\mathcal{N} \mathcal{T}_{X}}(\mathrm{FK}(A), \operatorname{FK}(B)) .
$$

Here, the subscript $\mathcal{N} \mathcal{T}_{X}$ denotes that Ext and Hom are taken in $\mathfrak{M o d}\left(\mathcal{N} \mathcal{T}_{X}\right)_{\mathrm{c}}^{\mathbb{Z} / 2}$, the target category of FK. 
(2) Let $A, B \in \mathcal{B}(X)$. Then $\mathrm{FK}(A) \cong \mathrm{FK}(B)$ implies that $A$ is $\mathrm{KK}_{X}$-equivalent to $B$.

(3) $X$ is of type $A$.

We will use that the negation of statement (2) in the theorem above is an obstruction to the existence of a UCT, which computes equivariant KK-theory in terms of filtrated K-theory. We prove that only spaces of type $A$ allow for a UCT by using functoriality of $\mathrm{KK}(X ; \ldots, \ldots)$ in the space variable to derive embedding results, which basically state that if a space $X$ has a subspace (or a retract) $Y$, for which there is no UCT then the same holds for $X$ itself. This shows that it is sufficient to check that there is no UCT for a small number of "bad apple" spaces. For these, it is known that the negation of statement (3) in the theorem above holds.

\section{Homological Algebra in Triangulated Categories}

The classical UCT of Rosenberg and Schochet is derived by using so called geometric resolutions of $C^{*}$-algebras. This technique of constructing ad hoc resolutions can be put in a more conceptual framework by regarding KK-theory as a triangulated category, where morphisms are given by KK-elements and the composition is given by the Kasparov product. This point of view has been introduced by R. Meyer and R. Nest in [27] to give an alternative description of the Baum-Connes conjecture. In [29], R. Meyer and R. Nest describe how homological algebra in triangulated categories can be approximated by homological algebra in usual abelian categories. In particular, they show that every "good" invariant has a unique modification that takes values in an abelian categorywith a little more work this allows for reducing the question whether there is a UCT short exact sequence for a given invariant to a simpler question: Do all objects arising as values of the modified invariant have projective dimension 1 ?

It seems to be more clear (at least to the author) how to construct the right resolutions in the algebraic world of abelian categories than in the $C^{*}$-algebraic world itself. This is the advantage of this approach as one moves on to more complicated versions of KKtheory for $C^{*}$-algebras with additional structure. The crux of the matter is to choose an invariant, which captures enough information but whose corresponding abelian category is still sufficiently tractable to construct the desired projective resolutions of length 1 .

Since this approach is the basis of all four results explained above, we will first work in the quite general context of triangulated categories with countable coproducts and (essential) idempotent suspension automorphisms. We will show for a large class of representable invariants how to reduce the question whether there is a UCT to a question about resolutions in a certain abelian category. We will then move on to the individual results, show how they fit in the general framework and give detailed explanations and proofs. 



\section{Background}

\section{Triangulated Categories and the Construction of Invariants}

Originally, triangulated categories were introduced as a concept to formalize structures which appear in stable homotopy theory and derived categories of abelian categories in homological algebra. A good general reference for triangulated categories is [31]. To the author's knowledge, their first explicit applications in non-commutative topology appeared in Andreas Thom's Thesis [45]. Having applications to KK-theory in mind, R. Meyer and R. Nest formulated the theory of homological algebra in triangulated categories building on work of D. Christensen [10], A. Beligiannis [3] and on earlier work in the context of relative homological algebra by S. Eilenberg and J. C. Moore [13].

We will assume the reader to be familiar with the definition and basic properties of triangulated categories. Let us just state that a triangulated category $\mathfrak{T}$ is a preadditive category, which is equipped with two pieces of extra data: A suspension automorphism $S$ and a class of so called exact triangles.

Let us now explain the prototypical example of a triangulated category as it will be used in this thesis: non-equivariant KK-theory. Here, the suspension automorphism is given by $S A:=\mathcal{C}_{0}(\mathbb{R}, A)$ for a separable $C^{*}$-algebra $A$. Bott periodicity shows that $S$ is an equivalence of categories, the small defect that $S$ is not an automorphism in the strict sense can be repaired by passing to a thickened category $\widetilde{\mathfrak{K} \mathfrak{K}}$, in which $\mathfrak{K} \mathfrak{K}$ sits as a full essential subcategory (see [27] 2.1 for details). For a $*$-homomorphism $\phi: A \rightarrow B$, one may form the mapping cone

$$
C_{\phi}:=\left\{(f, a) \in \mathcal{C}_{0}((0,1], B) \oplus A \mid f(1)=\phi(a)\right\}
$$

and the corresponding mapping cone triangle

$$
S B \stackrel{\iota_{\phi}}{\longrightarrow} C_{\phi} \stackrel{\epsilon_{\phi}}{\longrightarrow} A \stackrel{\phi}{\rightarrow} B, \quad \iota_{\phi}(g)=(g, 0), \epsilon_{\phi}(f, a)=f(1) .
$$

A diagram $S B^{\prime} \rightarrow C^{\prime} \rightarrow A^{\prime} \rightarrow B^{\prime}$ in $\mathfrak{K} \mathfrak{K}$ is an exact triangle if and only if it is isomorphic (in $\mathfrak{K} \mathfrak{K}$ ) to a mapping cone triangle. This is equivalent to the statement that $C^{\prime} \rightarrow A^{\prime} \rightarrow$ $B^{\prime}$ is isomorphic to a c.c.p. split extension of $C^{*}$-algebras (see[27] 2.2).

Also in the case of a general triangulated category $\mathfrak{T}$, we will write exact triangles in the form $S B \rightarrow C \rightarrow A \rightarrow B$. Note that this differs only in notation from the standard notation in triangulated categories: $S$ is the inverse of the suspension automorphism, which is used for instance in [31]. We introduce and follow this convention since exact triangles in this form show up naturally when working with KK-theory. 


\section{Background}

As mentioned in the introduction our approach of deriving a UCT short exact sequence relies on the machinery of homological algebra in triangulated categories. At this point we could spend a few pages to introduce the theory. But since there are already articles, such as [29], where this theory is nicely explained, we decided to outsource the explanation of the machinery. All statements in this section should be understandable without any knowledge about homological algebra in triangulated categories. For some proofs, however, we assume familiarity with the notions of [29].

We will write $C \in \in \mathfrak{C}$, if $\mathfrak{C}$ is a category and $C$ is an object in $\mathfrak{C}$. For $a \in \mathfrak{C}(A, B)$ and $b \in \mathfrak{C}(B, C)$, the composition $b \circ a$ will sometimes also be denoted by $a \cdot b \in \mathfrak{C}(A, C)$.

Let us assume that $\mathfrak{T}$ has countable coproducts. We would like to study $\mathfrak{T}$ by invariants, which take values in an abelian category $\mathfrak{A}$, i.e. by functors $F: \mathfrak{T} \rightarrow \mathfrak{A}$. A functor $F$ is called stable if $\mathfrak{A}$ is equipped with a suspension automorphism $\Sigma$ such that $F S=\Sigma F$ holds. A covariant functor $F$ is called homological if it maps an exact triangle $S B \rightarrow C \rightarrow A \rightarrow B$ to a long exact sequence

$$
\ldots \rightarrow \Sigma F(A) \rightarrow \Sigma F(B) \rightarrow F(C) \rightarrow F(A) \rightarrow F(B) \rightarrow \Sigma^{-1} F(C) \rightarrow \ldots
$$

Similarly, a contravariant functor $F$ is called cohomological if it maps an exact triangle $S B \rightarrow C \rightarrow A \rightarrow B$ to a long exact sequence

$$
\ldots \leftarrow \Sigma F(A) \leftarrow \Sigma F(B) \leftarrow F(C) \leftarrow F(A) \leftarrow F(B) \leftarrow \Sigma^{-1} F(C) \leftarrow \ldots
$$

Our aim in this chapter is to clarify what conditions on $F$ we really need in order to obtain a UCT short exact sequence, which computes the morphism groups of $\mathfrak{T}$ in terms of $F$, i.e. under which condition we can expect a short exact sequence of the form

$$
\operatorname{Ext}_{\mathfrak{A} \mathfrak{b}}^{1}(F(A), \Sigma F(B)) \longmapsto \mathfrak{T}(A, B) \rightarrow \operatorname{Hom}_{\mathfrak{A} \mathfrak{b}}(F(A), F(B)) .
$$

An obvious example will be given by Hom-functors. First, we need a definition.

Definition 5.1. Let $\mathfrak{T}_{*}$ be the category with the same objects as $\mathfrak{T}, \mathbb{Z}$-graded morphism groups $\mathfrak{T}_{*}(A, B)$, where $\mathfrak{T}_{n}(A, B), n \in \mathbb{N}$ is given by $\mathfrak{T}_{n}(A, B):=\mathfrak{T}\left(S^{n} A, B\right)$ and composition given by

$$
a \cdot b:=S^{m} a \cdot b
$$

for $a \in \mathfrak{T}_{n}(A, B)$ and $b \in \mathfrak{T}_{m}(B, C)$.

Definition 5.2. $A \in \in \mathfrak{T}$ is called $\omega$-compact, if $\mathfrak{T}(A, \ldots)$ commutes with direct sums. A countable family $\mathcal{A}$ of $\omega$-compact objects in $\mathfrak{T}$ such that for all $A \in \mathcal{A}, \mathfrak{T}(A, B)$ is countable for all $B \in \in \mathfrak{T}$, is called a c-family in $\mathfrak{T}$.

Let $\mathcal{A}$ be a c-family in $\mathfrak{T}$. We want to construct a functor $F_{\mathcal{A}}$, which takes values in an abelian category. Let us first describe the target category. Let $\mathfrak{T}_{*}^{\mathcal{A}}$ be the full subcategory of $\mathfrak{T}_{*}$ with objects $\mathcal{A}$. $\mathfrak{T}_{*}^{\mathcal{A}}$ is a small, preadditive category.

Definition 5.3. Let $\mathfrak{A} \mathfrak{b}_{c}^{\mathbb{Z}}$ denote the category of countable $\mathbb{Z}$-graded abelian groups. Let $\mathfrak{M o d}\left(\mathfrak{T}_{*}^{\mathcal{A}}\right)_{\mathrm{c}}^{\mathbb{Z}}$ be the category of countable $\mathbb{Z}$-graded $\mathfrak{T}_{*}^{\mathcal{A}}$-modules, i.e., the category whose 
objects consist of contravariant additive functors $M: \mathfrak{T}_{*}^{\mathcal{A}} \rightarrow \mathfrak{A b}_{c}^{\mathbb{Z}}$, which are grading preserving in the sense that for $a \in \mathfrak{T}_{n}(A, B), M(a): M(B) \rightarrow M(A)$ sends $M(B)[m]$ to $M(A)[m+n]$. Morphisms in $\mathfrak{M o d}\left(\mathfrak{T}_{*}^{\mathcal{A}}\right)_{\mathrm{c}}^{\mathbb{Z}}$ are given by grading preserving natural transformations $M \Rightarrow M^{\prime}$.

Performing pointwise constructions, it is not hard to check that $\mathfrak{M o d}\left(\mathfrak{T}_{*}^{\mathcal{A}}\right)_{\mathrm{c}}^{\mathbb{Z}}$ is abelian.

Remark 5.4. Let $\mathcal{R}_{\mathcal{A}}$ be the graded category ring of $\mathfrak{T}_{*}^{\mathcal{A}}$. $\mathcal{R}_{\mathcal{A}}$ is unital if and only if $\mathcal{A}$ is finite. In this case, the category of modules over $\mathfrak{T}_{*}^{\mathcal{A}}$ is equivalent to the category of $\mathcal{R}_{\mathcal{A}}$-modules.

Definition 5.5. Define $F_{\mathcal{A}}: \mathfrak{T} \rightarrow \mathfrak{M o d}\left(\mathfrak{T}_{*}^{\mathcal{A}}\right)_{\mathrm{c}}^{\mathbb{Z}}$ by $F_{\mathcal{A}}(B)(A)[n]:=\mathfrak{T}_{n}(A, B)$.

Composition on the left equips $F_{\mathcal{A}}(B)$ with the structure of a module over $\mathfrak{T}_{*}^{\mathcal{A}}$. Composition on the right shows that $F_{\mathcal{A}}$ is a covariant functor. $\mathfrak{M o d}\left(\mathfrak{T}_{*}^{\mathcal{A}}\right)_{\mathrm{c}}^{\mathbb{Z}}$ is equipped with a suspension automorphism $\Sigma$, which is given by $\Sigma M(A)[n]:=M(A)[n-1]$. It is clear that $F_{\mathcal{A}}$ is stable, Lemma 1.1.10 in [31] shows that $F_{\mathcal{A}}$ is homological. Thus for every c-family $\mathcal{A}$ in $\mathfrak{T}$, we have constructed an additive stable homological functor

$$
F_{\mathcal{A}}: \mathfrak{T} \rightarrow \mathfrak{M o d}\left(\mathfrak{T}_{*}^{\mathcal{A}}\right)_{\mathrm{c}}^{\mathbb{Z}}
$$

Lemma 5.6. Let $\mathcal{A}$ be a c-family in $\mathfrak{T}, n \in \mathbb{N}$ and $A \in \mathcal{A}$. Then $F_{\mathcal{A}}\left(S^{n} A\right) \in \in \mathfrak{M o d}\left(\mathfrak{T}_{*}^{\mathcal{A}}\right)_{\mathrm{c}}^{\mathbb{Z}}$ is projective. $\mathfrak{M o d}\left(\mathfrak{T}_{*}^{\mathcal{A}}\right)_{\mathrm{c}}^{\mathbb{Z}}$ has enough projective objects.

Proof. Let $M, N \in \in \mathfrak{M} \mathfrak{o d}\left(\mathfrak{T}_{*}^{\mathcal{A}}\right)_{\mathrm{c}}^{\mathbb{Z}}$, then by Yoneda's Lemma

$$
\mathfrak{M o d}\left(\mathfrak{T}_{*}^{\mathcal{A}}\right)_{\mathrm{c}}^{\mathbb{Z}}\left(F_{\mathcal{A}}\left(S^{n} A\right), M\right) \rightarrow M(A)[n], \quad \Phi \mapsto \Phi\left(\operatorname{id}_{S^{n} A}\right),
$$

is an isomorphism. Since $\Psi \in \mathfrak{M o d}\left(\mathfrak{T}_{*}^{\mathcal{A}}\right)_{\mathrm{c}}^{\mathbb{Z}}(N, M)$ is an epimorphism if and only if $\Psi(A): N(A) \rightarrow M(A)$ is onto for all $A \in \mathcal{A}$, this shows that $F_{\mathcal{A}}\left(S^{n} A\right)$ is projective. Furthermore, for every $A \in \mathcal{A}, n \in \mathbb{N}$ and $m \in M(A)[n]$, there is $\Phi_{m}: F_{\mathcal{A}}\left(S^{n} A\right) \rightarrow M$ such that $\Phi_{m}\left(\operatorname{id}_{S^{n} A}\right)=m$. Therefore

$$
\bigoplus_{A \in \mathcal{A}, n \in \mathbb{N}, m \in M(A)[n]} \Phi_{m}: \bigoplus_{A \in \mathcal{A}, n \in \mathbb{N}, m \in M(A)[n]} F_{\mathcal{A}}\left(S^{n} A\right) \rightarrow M
$$

is an epimorphism. This shows that $\mathfrak{M o d}\left(\mathfrak{T}_{*}^{\mathcal{A}}\right)_{\mathrm{c}}^{\mathbb{Z}}$ has enough projective objects.

As mentioned in the introduction, our aim is to translate homological algebra in the triangulated category $\mathfrak{T}$ to homological algebra in a suitable abelian category. Homological algebra in a triangulated category is always relative, that is we have to specify an ideal - a subclass $\mathfrak{J}$ of all morphisms in $\mathfrak{T}$, which is closed under composition [29]. We will only be concerned with ideals of the form $\mathfrak{J}:=\operatorname{ker} F$, where $\operatorname{ker} F(A, B):=\{f \in \mathfrak{T}(A, B) \mid F(f)=0\}$ for some stable homological functor $F$. In good cases, homological algebra in $\mathfrak{T}$ with respect to $\mathfrak{J}$ can be completely translated to homological algebra in an abelian category by means of a so called universal stable homological $\mathfrak{J}$-exact functor. 


\section{Background}

Definition 5.7. Let $\mathfrak{A}$ be an abelian category, $\mathfrak{J}$ an ideal in $\mathfrak{T}$ and $F: \mathfrak{T} \rightarrow \mathfrak{A}$ a stable homological functor. $F$ is the universal stable homological $\mathfrak{J}$-exact functor if for any other stable homological functor $G: \mathfrak{T} \rightarrow \mathfrak{A}^{\prime}$ with $G(f)=0 \forall f \in \in \mathfrak{J}$, there is a unique additive functor $\widetilde{G}: \mathfrak{A} \rightarrow \mathfrak{A}^{\prime}$ such that $G=\widetilde{G} \circ F$.

Lemma 5.8. Let $\mathcal{A}$ be a c-family in $\mathfrak{T}$. Then $F_{\mathcal{A}}$ is the universal stable homological

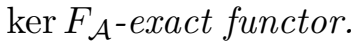

Proof. By [29] Theorem 3.39 and Remark 3.40 and our last Lemma (5.6), we only have to check that for every $A \in \mathcal{A}$ and every $n \in \mathbb{Z}$ there is an object $F_{\mathcal{A}}^{\perp}\left(F_{\mathcal{A}}\left(S^{n} A\right)\right) \in \in \mathfrak{T}$ such that

1. For every $B \in \in \mathfrak{T}$, there is an isomorphism

$$
\left.\left.\Theta_{B}: \mathfrak{M o d}^{\mathcal{A}} \mathfrak{T}_{*}^{\mathcal{Z}}\right)_{\mathrm{c}}^{\mathbb{Z}}\left(F_{\mathcal{A}}\left(S^{n} A\right)\right), F_{\mathcal{A}}(B)\right) \cong \mathfrak{T}\left(\left(F_{\mathcal{A}}^{\perp}\left(F_{\mathcal{A}}\left(S^{n} A\right)\right), B\right) .\right.
$$

2. $F_{\mathcal{A}}\left(\Theta_{S^{n} A}\left(\operatorname{id}_{F_{\mathcal{A}}\left(S^{n} A\right)}\right)\right)$ induces an isomorphism $F_{\mathcal{A}}\left(F_{\mathcal{A}}^{\perp}\left(F_{\mathcal{A}}\left(S^{n} A\right)\right)\right) \cong F_{\mathcal{A}}\left(S^{n} A\right)$.

Set $F_{\mathcal{A}}^{\perp}\left(F_{\mathcal{A}}\left(S^{n} A\right)\right):=S^{n} A$.

(1): Recall that $\left.\mathfrak{M o d}^{\prime} \mathfrak{T}_{*}^{\mathcal{A}}\right)_{\mathrm{C}}^{\mathbb{Z}}\left(F_{\mathcal{A}}\left(S^{n} A\right), M\right) \rightarrow M(A)[n], \Phi \mapsto \Phi\left(\operatorname{id}_{S^{n} A}\right)$ is an isomorphism, therefore, $\Theta_{B}$ is given by

$$
\left.\mathfrak{M o d}\left(\mathfrak{T}_{*}^{\mathcal{A}}\right)_{\mathrm{c}}^{\mathbb{Z}}\left(F_{\mathcal{A}}\left(S^{n} A\right)\right), F_{\mathcal{A}}(B)\right) \cong F_{\mathcal{A}}(B)(A)[n]=\mathfrak{T}\left(S^{n} A, B\right), \quad \Phi \mapsto \Phi\left(\operatorname{id}_{S^{n} A}\right)
$$

(2): We have $F_{\mathcal{A}}\left(F_{\mathcal{A}}^{\perp}\left(F_{\mathcal{A}}\left(S^{n} A\right)\right)\right)=F_{\mathcal{A}}\left(S^{n} A\right)$ and $F_{\mathcal{A}}\left(\Theta_{S^{n} A}\left(\operatorname{id}_{F_{\mathcal{A}}\left(S^{n} A\right)}\right)\right)=F_{\mathcal{A}}\left(\operatorname{id}_{S^{n} A}\right)$. This proves the lemma.

Corollary 5.9. Let $\mathcal{A}$ be a c-family in $\mathfrak{T}$ and $P \in \in \mathfrak{M o d}\left(\mathfrak{T}_{*}^{\mathcal{A}}\right)_{\mathrm{c}}^{\mathbb{Z}}$ projective, then there is $B \in \in \mathfrak{T}$ such that $F_{\mathcal{A}}(B) \cong P$.

Proof. This follows from Theorem 3.41 in [29], since $F_{\mathcal{A}}$ is the universal stable homological ker $F_{\mathcal{A}}$-exact functor.

\section{The Bootstrap Class}

Recall that we are looking for the general conditions, under which we can expect the existence of a UCT short exact sequence, i.e. of a short exact sequence of the form

$$
\operatorname{Ext}_{\mathfrak{A} \mathfrak{b}}^{1}(F(A), \Sigma F(B)) \succ \mathfrak{T}(A, B) \rightarrow \operatorname{Hom}_{\mathfrak{A} \mathfrak{b}}(F(A), F(B)) .
$$

As in the case of the usual UCT, we can expect this to hold only for objects $A$ in a certain bootstrap class.

Definition 6.1. Let $\mathcal{A}$ be a family of objects in $\mathfrak{T}$. The Bootstrap class $\mathfrak{B}_{\mathcal{A}}$ is the localizing subcategory generated by $\mathcal{A}$, i.e., the smallest subclass $\mathcal{C}$ of objects of $\mathfrak{T}$ which is closed under the following operations: 
1. (suspension) If $A$ is in $\mathcal{C}$, then $S A$ and $S^{-1} A$ are in $\mathcal{C}$.

2. (exact triangles) If $S B \rightarrow C \rightarrow A \rightarrow B$ is an exact triangle and two of the three objects $A, B$ and $C$ are in $\mathcal{C}$, then so is the third.

3. ( direct sums) If $\left(A_{i}\right)_{i \in I}$ is a countable family of objects in $\mathcal{C}$, then so is $\bigoplus_{I} A_{i}$.

4. (retracts) If $B$ is in $\mathcal{C}$ and there are $f: A \rightarrow B$ and $g: B \rightarrow A$ such that $f \cdot g=\operatorname{id}_{A}$, then $A$ is in $\mathcal{C}$.

If $\mathcal{A}$ consists only of one object $A$, we will also write $\mathfrak{B}_{A}$ instead of $\mathfrak{B}_{\mathcal{A}}$.

Lemma 6.2. Let $\mathcal{A}$ be a c-family of objects in $\mathfrak{T}$. Then $\mathfrak{B}_{\bigoplus_{\in \mathcal{A}} A}=\mathfrak{B}_{\mathcal{A}}$.

Proof. We obviously have that $\mathfrak{B}_{\bigoplus_{A \in \mathcal{A}} A}$ is a subclass of $\mathfrak{B}_{\mathcal{A}}$. The other direction follows by the fact that $\mathfrak{B}_{\mathcal{A}}$ as a localizing subcategory is automatically thick [31], i.e. that $B \oplus C \in \in \mathfrak{B}_{\mathcal{A}}$ implies $B \in \in \mathfrak{B}_{\mathcal{A}}$.

The next lemma tells us that the objects in the bootstrap class $\mathfrak{B}_{\mathcal{A}}$ are "orthogonal" to the class of objects on which $F_{\mathcal{A}}$ vanishes.

Lemma 6.3. Let $\mathcal{A}$ be a countable family of objects in $\mathfrak{T}$ and $B$ in $\mathfrak{B}_{A}$. Then $B$ has the following property: If $C \in \in \mathfrak{T}$ such that $F_{\mathcal{A}}(C)=0$ then $\mathfrak{T}_{*}(B, C)=0$.

Proof. Let $\mathfrak{B}^{\prime}$ be the class of of objects $B \in \in \mathfrak{T}$ such that $F_{\mathcal{A}}(C)=0$ implies $\mathfrak{T}(B, C)=$ $0 . \mathfrak{B}^{\prime}$ is closed under taking suspensions, exact triangles (five lemma), retracts and countable direct sums and obviously contains $\mathcal{A}$, hence it contains $\mathfrak{B}_{A}$.

Under a certain regularity condition on the objects in $\mathcal{A}$, the property of the last lemma completely characterizes the bootstrap class.

Note that the five lemma implies that the full subcategory of $\omega$-compact objects is thick, i.e. is closed under suspensions, exact triangles and countable coproducts.

Proposition 6.4. Let $\mathcal{A}$ be a c-family in $\mathfrak{T}$ and $B \in \in \mathfrak{T}$. The following statements are equivalent:

(1) $B$ is in $\mathfrak{B}_{\mathcal{A}}$.

(2) If $C \in \in \mathfrak{T}$ such that $F_{\mathcal{A}}(C)=0$ then $\mathfrak{T}(B, C)=0$.

Proof. We only have to show $(2) \Rightarrow(1)$. [27] Theorem 6.1 , shows that $\mathfrak{T}(\ldots, B)$ restricted to the triangulated subcategory $\mathfrak{B}_{\mathcal{A}}$ is representable, i.e. there is a $\widetilde{B}$ in $\mathfrak{B}_{\mathcal{A}}$ and an isomorphism of (restricted) functors $T: \mathfrak{T}(\ldots, B)_{\mid \mathfrak{B}_{\mathcal{A}}} \cong \mathfrak{T}(\ldots, \widetilde{B})_{\mid \mathfrak{B}_{A}}$. Set $\phi:=T_{B}\left(\operatorname{id}_{B}\right) \in \mathfrak{T}(B, \widetilde{B})$ and let $C_{\phi}$ be the mapping cone of $\phi$. Since $\phi$ is an $F_{\mathcal{A}^{-}}$ equivalence by construction, $F_{\mathcal{A}}\left(C_{\phi}\right)=0$. Therefore, $\mathfrak{T}_{*}\left(B, C_{\phi}\right)=0$. We also have $\mathfrak{T}_{*}\left(\widetilde{B}, C_{\phi}\right)=0$. Since $\mathfrak{T}_{*}\left(\ldots, C_{\phi}\right)$ is cohomological, this shows that $\mathfrak{T}_{*}\left(C_{\phi}, C_{\phi}\right)=0$, i.e. that $C_{\phi} \cong 0$ in $\mathfrak{T}$. Therefore, $\phi$ is an isomorphism.

In applications to KK-theory, it is often desirable to have a more explicit characterization of the objects in the corresponding bootstrap class. This can be done in most cases of interest as the reader will see once we are dealing with applications. 


\section{A General UCT Criterion}

We present a general criterion for the existence of a UCT short exact sequence for invariants of the form $F_{\mathcal{A}}$, which only involves the bootstrap class $\mathfrak{B}_{\mathcal{A}}$ and projective resolutions in the target category of $F_{\mathcal{A}}$.

Theorem 7.1. Let $\mathcal{A}$ be a c-family of objects in $\mathfrak{T}$ and $B \in \in \mathfrak{T}$. Assume that

(1) $B \in \in \mathfrak{B}_{\mathcal{A}}$;

(2) $F_{\mathcal{A}}(B)$ has a projective resolution of length 1 in $\mathfrak{M o d}\left(\mathfrak{T}_{*}^{\mathcal{A}}\right)_{\mathrm{c}}^{\mathbb{Z}}$.

Then, for every $C \in \in \mathfrak{T}$, there is a grading preserving short exact UCT sequence:

$$
\operatorname{Ext}_{\mathfrak{M} \mathfrak{o d}\left(\mathfrak{T}_{*}^{\mathcal{A}}\right)_{\mathrm{c}}^{\mathbb{Z}}}^{1}\left(\Sigma F_{\mathcal{A}}(B), F_{\mathcal{A}}(C)\right) \longmapsto \mathfrak{T}(B, C) \rightarrow \operatorname{Hom}_{\mathfrak{M}_{\mathfrak{o d}}\left(\mathfrak{T}_{*}^{\mathcal{A}}\right)_{\mathrm{c}}^{\mathbb{Z}}}\left(F_{\mathcal{A}}(B), F_{\mathcal{A}}(C)\right) .
$$

Proof. Set $\mathfrak{J}:=\operatorname{ker}\left(F_{A}\right)$, i.e. $\mathfrak{J}(A, B)=\left\{f \in \mathfrak{T}(A, B) \mid F_{A}(f)=0\right\}$, then $\mathfrak{J}$ is a homological ideal in the sense of Definition 2.20 of [29]. By definition of $\mathfrak{J}, F_{A}$ is $\mathfrak{J}$-exact.

We already know that $F_{\mathcal{A}}$ is the universal $\operatorname{ker} F_{\mathcal{A}}$-exact stable homological functor. By [29] Theorem 3.41, assumption (2) implies that $B$ has a ker $F_{\mathcal{A}}$-projective resolution of length 1 . The same theorem states that there are natural isomorphisms

$$
\operatorname{Ext}_{\mathfrak{M} \mathfrak{o r}\left(\mathfrak{T}_{*}^{\mathcal{A}}\right)_{\mathrm{C}}^{\mathbb{Z}}}^{n}\left(F_{\mathcal{A}}(B), F_{\mathcal{A}}(C)\right) \cong \operatorname{Ext}_{\mathfrak{T}, \operatorname{ker} F_{\mathcal{A}}}^{n}(B, C) \text { for all } n \in \mathbb{N} \text {. }
$$

[29] Lemma 3.2 and Lemma 6.3 of the present chapter tell us that assumption (1) implies that $\mathfrak{T}(B, C)=0$ for all $\operatorname{ker} F_{\mathcal{A}}$-contractible $C$. Therefore, the statement follows by [29] Theorem 4.4. 


\section{Equivariant KK-theory and the UCT for the Action of a Cyclic Group of Prime Order}

The main aim of this chapter is to prove an equivariant Universal Coefficient Theorem for $C^{*}$-algebras equipped with an action of $\mathbf{C}(p)$. More precisely, we will define $\mathrm{EK}^{\mathbf{C}(p)}$ as the invariant associated with the family $\mathcal{A}:=\left\{\mathbb{C}, \mathcal{C}(\mathbf{C}(p)), C_{u}\right\}$ in $\mathfrak{K} \mathfrak{K} \mathbf{C}(p)$. Where $C_{u}$ denotes the mapping cone of the unital embedding of $\mathbb{C}$ into $\mathcal{C}(\mathbf{C}(p))$. EK ${ }^{\mathbf{C}(p)}$ takes values in the category of countable $\mathbb{Z} / 2$-graded modules over $\mathcal{R}$-the category ring of $\mathfrak{K} \mathfrak{K} \mathbf{C}(p)$ restricted to $\mathcal{A}$. For separable $\mathbf{C}(p)$ - $C^{*}$-algebras $A$ and $B$ with $A$ being in a certain bootstrap class $\mathfrak{B}^{\mathrm{C}(p)}$, we will prove the existence of a short exact UCT sequence

$$
\operatorname{Ext}_{\mathcal{R}}^{1}\left(\operatorname{EK}^{\mathbf{C}(p)}(S A), \operatorname{EK}^{\mathbf{C}(p)}(B)\right) \longmapsto \operatorname{KK}_{*}^{\mathbf{C}(p)}(A, B) \rightarrow \operatorname{Hom}_{\mathcal{R}}\left(\operatorname{EK}^{\mathbf{C}(p)}(A), \operatorname{EK}^{\mathbf{C}(p)}(B)\right) .
$$

We will also show that at least all $\mathbf{C}(p)$ - $C^{*}$-algebras of type I are in $\mathfrak{B}^{\mathbf{C}(p)}$.

This chapter is organized as follows: In Section 8, we explain for a locally compact group $G$ how to view $\mathrm{KK}^{G}$ as a triangulated category with countable coproducts. We furthermore derive some properties of the Hom-like invariants $F_{\mathcal{A}}$ such as a ConnesThom isomorphism and a Pimsner-Voiculescu exact sequence. Then in 9, we compare our definition of the bootstrap class with the definition of Rosenberg and Schochet and show that in the case $G=\mathbf{C}(p)$ all type I algebras are in the corresponding bootstrap class. In Section 10, we introduce the invariant $\mathrm{EK}^{\mathbf{C}(p)}$ as a module over a category ring $\mathcal{R}$ as a special case of the general construction. In Section 11, we will calculate the ring $\mathcal{R}$ in terms of generators and relations and use this description in Section 12 to show that there is a UCT short exact sequence for $\mathrm{EK}^{\mathbf{C}(p)}$ by proving that every module of the form $\operatorname{EK}^{\mathbf{C}(p)}(A)$ has projective dimension 1.

\section{Equivariant KK-theory as a Triangulated Category}

\subsection{The Categories $\mathfrak{K} \mathfrak{K}^{G}$ and $\mathfrak{K}_{\mathfrak{K}_{*}^{G}}^{G}$}

For a locally compact group $G$, let $\mathfrak{K}_{\mathfrak{K}}^{G}$ denote the category, which has separable $G-C^{*}$ algebras as objects and in which the set of morphisms from $A$ to $B$ is equal to $\operatorname{KK}^{G}(A, B)$. Composition is given by the Kasparov product. $\mathfrak{K}_{\mathfrak{K}}^{G}$ is a preadditive category, which has countable coproducts, it also carries the structure of a triangulated category (for a proof consult the Appendix of [27]).

As in the non-equivariant case, the suspension automorphism $S$ of $\mathfrak{K}_{\mathfrak{K}}^{G}$ is given by taking the outer tensor product with $\mathcal{C}_{0}(\mathbb{R})$. A triangle is exact if and only if it is 
isomorphic to a mapping cone triangle. This is equivalent to the statement that it is isomorphic to a $G$-equivariantly c.c.p. split extension ([27] Section 2.1.).

Following Definition 5.1, we may form $\mathfrak{K}_{\mathfrak{K}_{*}^{G}}$. Due to Bott periodicity, it suffices to work with a slightly simpler $\mathbb{Z} / 2$-graded variant: Define

$$
\mathrm{KK}_{*}^{G}(A, B):=\mathrm{KK}_{0}^{G}(A, B) \oplus \mathrm{KK}_{1}^{G}(A, B)
$$

Let $\mathcal{B} \in \mathrm{KK}^{G}\left(\mathbb{C}, S^{2}\right)$ and $\mathcal{D} \in \mathrm{KK}^{G}\left(S^{2}, \mathbb{C}\right)$ denote the usual Bott and Dirac element and for a $G$ - $C^{*}$-algebra $A$ define isomorphisms $\mathcal{B}_{A}:=\mathcal{B} \otimes_{\mathbb{C}} 1_{A} \in \mathrm{KK}^{G}\left(A, S^{2} A\right)$ and $\mathcal{D}_{A}:=\mathcal{D} \otimes_{\mathbb{C}} 1_{A} \in \mathrm{KK}^{G}\left(S^{2} A, A\right)$.

Definition 8.1. Let $A, B$ and $C$ be separable (trivially graded) $G$ - $C^{*}$-algebras, $i, j \in$ $\{0,1\}$ and $x \in \operatorname{KK}_{i}^{G}(A, B), y \in \operatorname{KK}_{j}^{G}(B, C)$. Set $k=i+j \bmod 2$. Define a product

$$
\mathrm{KK}_{i}^{G}(A, B) \otimes \mathrm{KK}_{j}^{G}(B, C) \rightarrow \mathrm{KK}_{k}^{G}(A, C)
$$

by

$$
x \cdot y:= \begin{cases}x \otimes_{B} y & \text { if } j=0 \\ S x \otimes_{S B} y & \text { if } i=0, j=1 \\ \mathcal{B}_{A} \otimes_{S^{2} B} S x \otimes_{S B} y & \text { if } i=0, j=1\end{cases}
$$

Using that $1_{S} \otimes_{\mathbb{C}} \mathcal{B}=\mathcal{B} \otimes_{\mathbb{C}} 1_{S}$ (which follows from the fact that $\mathbb{R}^{3} \rightarrow \mathbb{R}^{3},(x, y, z) \mapsto$ $(z, x, y)$ is homotopic to the identity), it is easy to check that this product is associative. Of course $1_{A}$ acts as the identity on $A$. We obtain a category, which is equivalent to the $\mathbb{Z}$-graded version of definition 5.1. It will also be denoted by $\mathfrak{K}_{*}^{G}$.

\subsection{Properties of the Hom-like Invariants in the $G$-equivariant Case}

Let $\mathcal{A}$ be a c-family of objects in $\mathfrak{K}_{\mathfrak{K}}^{G}$. In 5.5 , we constructed an invariant $F_{\mathcal{A}}$. For instance, if $G$ is compact and we set $\mathcal{A}=\{\mathbb{C}\}$ with the trivial grading, we obtain equivariant K-theory with its module structure over the complex representation ring $R(G)$.

Thanks to Bott periodicity, the long exact sequence is 6-periodic, i.e., if $S A \rightarrow C \rightarrow$ $A \rightarrow B$ is an exact triangle (for example, if $C \rightarrow A \rightarrow B$ is an equivariantly c.c.p. split extension) then there is a 6 -term exact sequence

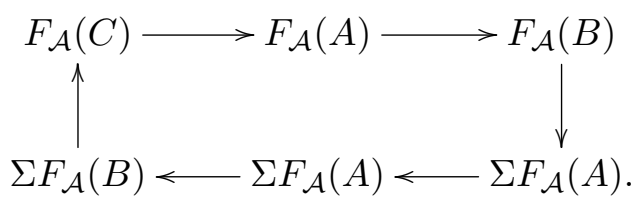

Recall that K-theory is exact, even if it is applied to non c.c.p. split extensions. If we restrict to compact $G$ and a family $\mathcal{A}$ consisting of nuclear $C^{*}$-algebras, the same holds for $F_{\mathcal{A}}$ : 
Lemma 8.2. Let $G$ be compact and all $A \in \mathcal{A}$ nuclear. If $B_{1} \longmapsto B_{2} \rightarrow B_{3}$ is a $G$-equivariant extension, then there is a natural 6 -term exact sequence

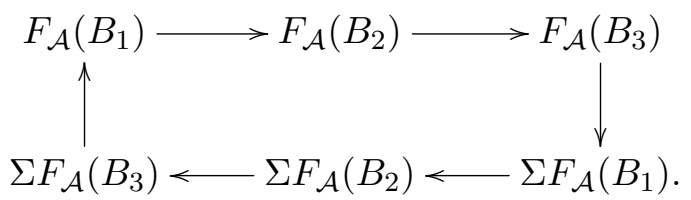

Proof. This follows from the main result of [24] and is also contained in [42] .

The statement of the lemma is true in the more general context of $G$ being strongly $\mathrm{K}$ amenable and $A$ being nuclear in equivariant $\mathrm{K}$-theory, for example if $G$ is discrete and amenable, then it is strongly K-amenable (see [24] for an explanation of these notions and a proof of this result).

Next we will derive analogues of the Thom isomorphism and the Pimsner-Voiculescu exact sequence. Let $G_{1}$ and $G_{2}$ be locally compact groups. If $B \in \in \mathfrak{K} \mathfrak{K}^{G_{1} \times G_{2}}$, then taking the partial crossed product ([23] 3.11) yields $G_{2} \ltimes A \in \in \mathfrak{K}_{\mathfrak{K}}^{G}$. Let furthermore $\eta: \mathfrak{K} \mathfrak{K}^{G_{1} \times G_{2}} \rightarrow \mathfrak{K}_{\mathfrak{K}}{ }^{G_{1}}$ denote the functor which is given by forgetting the $G_{2}$-action.

Proposition 8.3 (Thom isomorphism). Let $B \in \in \mathfrak{K}_{\mathfrak{K}}^{G \times \mathbb{R}}$, then there is a $\mathrm{KK}^{G}$ equivalence

$$
\eta S B \cong B \ltimes \mathbb{R} .
$$

This isomorphism passes to any functor, which is defined on $\mathfrak{K}_{\mathfrak{K}}^{G}$, for example by applying $F_{\mathcal{A}}$, we obtain a Thom isomorphism $\Sigma F_{\mathcal{A}}(B) \cong F_{\mathcal{A}}(\mathbb{R} \ltimes B)$.

Proof. Equip $\mathcal{C}_{0}(\mathbb{R}) \in \in \mathfrak{K}_{\mathfrak{K}}^{G \times \mathbb{R}}$ with an $\mathbb{R}$-action by translation and trivial $G$-action. First consider $\mathcal{C}_{0}(\mathbb{R})$ as an $\mathbb{R}$-algebra by forgetting the $G$-action. By [23] Theorem 5.7 and Theorem 5.9 applied to $\mathbb{R}$, we obtain a $\mathrm{KK}^{\mathbb{R}}$-equivalence $\mathbb{C} \cong \mathcal{C}_{\tau}(\mathbb{R})=\mathbb{C}_{1} \otimes \mathcal{C}_{0}(\mathbb{R})$, where $\mathbb{C}_{1}$ denotes the first Clifford algebra with trivial $\mathbb{R}$-action. Usual Bott periodicity yields $\mathbb{C} \cong S \mathcal{C}_{0}(\mathbb{R})$ in $\mathfrak{K}_{\mathfrak{K}^{\mathbb{R}}}$, or equivalently, $S \cong \mathcal{C}_{0}(\mathbb{R})$. Pullback via the projection $G \times \mathbb{R} \rightarrow \mathbb{R}$ gives $S \cong \mathcal{C}_{0}(\mathbb{R})$ in $\mathfrak{K} \mathfrak{K}^{G \times \mathbb{R}}$. Applying the exterior product with $1_{B}$ yields a $\mathrm{KK}^{G \times \mathbb{R}}$-equivalence $S B \cong \mathcal{C}_{0}(\mathbb{R}, B)$ (Here, $G \times \mathbb{R}$ acts on $\mathcal{C}_{0}(\mathbb{R}, B)$ diagonally via the the identification $\left.\mathcal{C}_{0}(\mathbb{R}, B) \cong \mathcal{C}_{0}(\mathbb{R}) \otimes B\right)$. Let $\widetilde{B}$ denote $B$ with $G$ acting as before but with trivial $\mathbb{R}$-action. Then

$$
\mathcal{C}_{0}(\mathbb{R}, \widetilde{B}) \rightarrow \mathcal{C}_{0}(\mathbb{R}, B), \quad f \mapsto(t \mapsto(e, t) \cdot f(t))
$$

yields $G \times \mathbb{R}$-equivariant $*$-isomorphism. Hence we obtain a chain of $\mathrm{KK}^{G \times \mathbb{R}_{\text {-equivalences }}}$

$$
S B \cong \mathcal{C}_{0}(\mathbb{R}, B) \cong \mathcal{C}_{0}(\mathbb{R}, \widetilde{B}) \cong S \widetilde{B}
$$

or, equivalently, $B \cong \widetilde{B}$ in $\mathfrak{K}_{\mathfrak{K}}^{G \times \mathbb{R}}$. Now applying the partial crossed product with $\mathbb{R}$ yields the claim since $C^{*} \mathbb{R} \cong S$.

Proposition 8.4 (Pimsner-Voiculescu exact triangle). Let $B \in \in \mathfrak{K} \mathfrak{K}^{G \times \mathbb{Z}}$, then there is an exact triangle

$$
\eta B \rightarrow \eta B \rightarrow \mathbb{Z} \ltimes B \rightarrow \eta S B
$$


Applying $F_{\mathcal{A}}$ or any other (co-)homological functor yields a Pimsner-Voiculescu type exact sequence.

Proof. Let $G \times \mathbb{Z}$ act on $\mathcal{C}_{0}(\mathbb{R}, B)$ diagonally with $G$ acting trivially and $\mathbb{Z}$ acting by translation on $\mathbb{R}$ and similarly for $\mathcal{C}_{0}(\mathbb{Z}, B)$. Restriction provides a surjection $\mathcal{C}_{0}(\mathbb{R}, B) \rightarrow$ $\mathcal{C}_{0}(\mathbb{Z}, B)$, whose kernel may be identified with $S \mathcal{C}_{0}(\mathbb{Z}, B)$. The resulting extension

$$
S \mathcal{C}_{0}(\mathbb{Z}, B) \longmapsto \mathcal{C}_{0}(\mathbb{R}, B) \rightarrow \mathcal{C}_{0}(\mathbb{Z}, B)
$$

is $\mathbb{Z} \times G$-equivariantly c.c.p. split and hence provides an exact triangle in $\mathfrak{K} \mathfrak{K}^{\mathbb{Z} \times G}$.

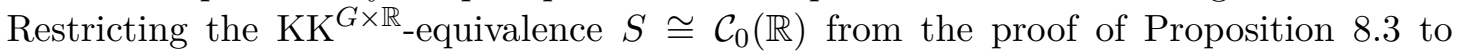
$G \times \mathbb{Z}$ and tensoring with $1_{B}$ yields a $\mathrm{KK}^{G \times \mathbb{Z}}$-equivalence $\mathcal{C}_{0}(\mathbb{R}, B) \cong S B$. Let $\widetilde{B}$ denote $B$ with $G$ acting as before but with trivial $\mathbb{Z}$-action.

$$
\mathcal{C}_{0}(\mathbb{Z}, \widetilde{B}) \rightarrow \mathcal{C}_{0}(\mathbb{Z}, B), \quad f \mapsto(n \mapsto(e, n) \cdot f(n)),
$$

yields a $G \times \mathbb{Z}$-equivariant $*$-isomorphism. Hence there is an exact triangle

$$
S \mathcal{C}_{0}(\mathbb{Z}, \widetilde{B}) \longmapsto S B \rightarrow \mathcal{C}_{0}(\mathbb{Z}, \widetilde{B}) .
$$

Now we can apply the partial crossed product with $\mathbb{Z}$ and shift the resulting exact triangle to an exact triangle of the desired form.

\section{A close Look at the Bootstrap Class}

Recall that $\mathfrak{B}_{\mathcal{A}}$ is the localizing subcategory of $\mathfrak{K}_{\mathfrak{K}}^{G}$ generated by $A \in \mathcal{A}$. As an example, consider $\mathcal{F}=\left(\mathcal{C}_{0}(G / H)\right)_{H}$ closed subgroup of $G$. Then $\mathfrak{B}_{\mathcal{F}}$ can be thought of as a KK-analogue of the category of (pointed) $G$-CW-complexes (as for instance introduced in [25]), i.e., all separable $G$ - $C^{*}$-algebras which can be constructed from basic building blocks of the form $\mathcal{C}_{0}(G / H)$ via the operations of $\mathrm{KK}^{G}$-equivalence, countable direct sums, suspensions and exact triangles. Taking suspensions is the direct analogue of the topological suspension functor, countable direct sums and exact triangles correspond to the gluing procedure for $G$-CW-complexes. Of course, since we are including $\mathrm{KK}^{G}$ equivalences, the bootstrap class contains much more than just (continuous functions on) $G$-CW-complexes.

Let us now connect our notion of a bootstrap class to the one of Rosenberg and Schochet defined in [40]. Note that Rosenberg and Schochet only consider nuclear G$C^{*}$-algebras. A useful property of the subclass of nuclear $G$ - $C^{*}$-algebras is that for a compact $G$ we do not have to distinguish between extensions and admissible extensions, a fact that is well known for the non-equivariant case and easily extends to the equivariant setting if $G$ is compact.

Lemma 9.1. Let $G$ be a compact topological group,

$$
J \longmapsto A \rightarrow Q
$$

a $G$-equivariant extension and $Q$ a nuclear $G$ - $C^{*}$-algebra, then there is a $G$-equivariant c.c.p. splitting $\sigma$. 
Proof. By [9], there is a (not necessarily equivariant) c.p. splitting section $s: Q \rightarrow A$ of norm at most 1 . An equivariant splitting is given by

$$
\sigma(q):=\int_{G} g^{-1} s(g q) \mathrm{d} \mu_{G} .
$$

Let $\mathcal{C}$ be a class of nuclear $G$ - $C^{*}$-algebras. Consider the following closure properties:

1. Let $0 \rightarrow A \rightarrow B \rightarrow C \rightarrow 0$ be a short exact sequence of $G$ - $C^{*}$-algebras. If two of the three algebras are in $\mathcal{C}$, then the third is also in $\mathcal{C}$.

2. If $\left(A_{n}, \alpha_{n}^{m}\right)$ is a countable inductive system of $G$ - $C^{*}$-algebras in $\mathcal{C}$, then $A_{\infty}:=$ $\underset{\lim }{\longrightarrow}\left(A_{n}, \alpha_{n}^{m}\right)$ is in $\mathcal{C}$.

3. If $(A, \alpha)$ is in $\mathcal{C}$ and $(A, \alpha)$ is exterior equivalent to $\left(A, \alpha^{\prime}\right)$, then $\left(A, \alpha^{\prime}\right)$ is in $\mathcal{C}$.

4. If $A$ is in $\mathcal{C}$ and $A$ is $G$-stably isomorphic to $B$, then $B$ is in $\mathcal{C}$.

5. If $A$ in $\mathcal{C}$ admits an action of $\mathbb{R}$ (or $\mathbb{Z}$ ) that commutes with the $G$ action, then $\mathbb{R} \ltimes A$ (or $\mathbb{Z} \ltimes A$, respectively) is in $\mathcal{C}$

Definition 9.2. Let $\mathcal{F}$ be a family of nuclear $G$ - $C^{*}$-algebras. Define $\langle\langle\mathcal{F}\rangle\rangle$ to be the smallest subclass of all subclasses of nuclear $G$-C $C^{*}$-algebras that contain $\mathcal{F}$ and have the closure properties (1)-(5).

Proposition 9.3. Let $G$ be a compact group and $\mathcal{F}$ be a family of nuclear $G$-C $C^{*}$-algebras. Then

$$
\langle\langle\mathcal{F}\rangle\rangle \subseteq \mathfrak{B}_{\mathcal{F}} .
$$

Proof. Let $\mathcal{C}$ be the class of all nuclear $G$-C $C^{*}$-algebras in $\mathfrak{B}_{\mathcal{F}}$. We have to show that $\mathcal{C}$ has the closure properties (1)-(5). This is true for (3) and (4), since $\mathfrak{B}_{\mathcal{F}}$ is closed under $\mathrm{KK}^{G}$-equivalence and stabilization preserves nuclearity. Since crossed products by $\mathbb{Z}$ and $\mathbb{R}$ preserve nuclearity, $\mathcal{C}$ is closed under (5) by Propositions 8.4 and 8.3. (1) holds by Lemma 9 and since nuclearity passes to quotients, ideals and is preserved under extensions ([35], the remark following efinition 11.7). To prove (2), let $\left(A_{n}, \alpha_{n}^{m}\right)$ be a countable inductive system of $G$ - $C^{*}$-algebras in $\mathcal{C}$. Recall from [27] Section 2.4., that there is a homotopy limit ho- $\lim _{\longrightarrow}\left(A_{n}, \alpha_{n}^{m}\right)$, which is, by definition, part of an exact triangle

$$
S\left(\text { ho- } \lim _{\longrightarrow}\left(A_{n}, \alpha_{n}^{m}\right)\right) \rightarrow \bigoplus_{\mathbb{N}} A_{n} \rightarrow \bigoplus_{\mathbb{N}} A_{n} \rightarrow \text { ho- } \lim _{\longrightarrow}\left(A_{n}, \alpha_{n}^{m}\right)
$$

and that ho- $\lim _{\longrightarrow}\left(A_{n}, \alpha_{n}^{m}\right)$ is $\mathrm{KK}^{G}$-equivalent to $A_{\infty}$ if the extension of $G$ - $C^{*}$-algebras

$$
T\left(A_{n}, \alpha_{n}^{m}\right) \longmapsto \widetilde{T}\left(A_{n}, \alpha_{n}^{m}\right) \rightarrow A_{\infty}
$$

is admissible, i.e., has a $G$-equivariant c.c.p. split. Here, $T\left(A_{n}, \alpha_{n}^{m}\right)$ and $\widetilde{T}\left(A_{n}, \alpha_{n}^{m}\right)$ are mapping telescope constructions, for the exact definition see [27] p. 11-12. Since a direct limit of nuclear $C^{*}$-algebras is again nuclear ([7], remark to Exercise 2.3.7.), we see that $T\left(A_{n}, \alpha_{n}^{m}\right) \longmapsto \widetilde{T}\left(A_{n}, \alpha_{n}^{m}\right) \rightarrow A_{\infty}$ is admissible by Lemma 9 . This gives a $\mathrm{KK}^{G}$-equivalence $A_{\infty} \cong$ ho- $\lim _{\longrightarrow}\left(A_{n}, \alpha_{n}^{m}\right)$. Since $\mathfrak{B}_{\mathcal{F}}$ is closed under direct sums, exact triangles and $\mathrm{KK}^{G}$-equivalences, this shows that $A_{\infty}$ is in $\mathcal{C}$. 
As in [40], let $\mathcal{A}_{G}$ be the class of all separable abelian $G$ - $C^{*}$-algebras. Rosenberg and Schochet's version of the bootstrap class is $\left\langle\left\langle\mathcal{A}_{G}\right\rangle\right\rangle$. They prove the following useful theorem:

Theorem 9.4. Let $G$ be a compact Lie group and $\mathcal{F}$ a collection of $G$-C $C^{*}$-algebras such that for all closed subgroups $H$ of $G$ and all continuous trace $G$ - $C^{*}$-algebras $A$ with spectrum $\widehat{A}=G / H$ ( $G$ acting by translation $), A \in \mathcal{F}$. If $B$ is a type $I C^{*}$-algebra and $\beta$ a $G$-action on $B$, then $(B, \beta)$ is in $\langle\langle\mathcal{F}\rangle\rangle$.

Proof. See [40], Theorem 2.8.

The same techniques as in the proof of the last theorem may be used to connect Rosenberg's and Schochet's notion of a bootstrap class with our definition.

Theorem 9.5. Let $G$ be a compact (not necessarily connected) Lie group and

$$
\mathcal{F}_{G}:=\left(\mathcal{C}_{0}(G / H)\right)_{H} \text { closed subgroup of } G .
$$

Then

$$
\left\langle\left\langle\mathcal{A}_{G}\right\rangle\right\rangle \subseteq \mathfrak{B}_{\mathcal{F}_{G}} .
$$

Proof. By Proposition 9.3 it is sufficient to show that every separable abelian $G-C^{*}$ algebra is contained in $\left\langle\left\langle\mathcal{F}_{G}\right\rangle\right\rangle$, i.e., that $\left\langle\left\langle\mathcal{F}_{G}\right\rangle\right\rangle=\left\langle\left\langle\mathcal{A}_{G}\right\rangle\right\rangle$. The arguments in the proof of Theorem 2.8. p. 19 in [40] can be applied verbatim, just replace "type I" and "continuous trace" by "abelian."

Note that $\left\langle\left\langle\mathcal{A}_{G}\right\rangle\right\rangle \neq \mathfrak{B}_{\mathcal{F}_{G}}$ just because $\left\langle\left\langle\mathcal{A}_{G}\right\rangle\right\rangle$ consists of nuclear $C^{*}$-algebras by definition, and $\mathfrak{B}_{\mathcal{F}_{G}}$ also contains non-nuclear $C^{*}$-algebras, which are KK-equivalent to 0 .

Let us now consider the case $G=\mathbf{C}(p)$. Let $\mathfrak{B}^{\mathbf{C}(p)}$ denote the bootstrap class $\mathfrak{B}_{\{\mathbb{C}, \mathcal{C} \mathbf{C}(p)\}}$. We will use the Theorem of Rosenberg and Schochet to show that at least all type I $C^{*}$-algebras are in $\mathfrak{B}^{\mathrm{C}(p)}$.

Corollary 9.6. Let $A$ be a type $I C^{*}$-algebra and $\alpha$ a $\mathbf{C}(p)$-action on $A$. Then $(A, \alpha)$ is in $\mathfrak{B}^{\mathbf{C}(p)}$.

Proof. In view of Lemma 9.3, Theorem 9.4 and the fact that $\langle\langle\langle\mathcal{F}\rangle\rangle\rangle\rangle=\langle\langle\mathcal{F}\rangle\rangle$, we only have to show that $\mathcal{F}:=\langle\langle\{\mathbb{C}, \mathcal{C} \mathbf{C}(p)\}\rangle\rangle$ fulfills the assumption of Theorem 9.4. If $A$ is a continuous trace $\mathbf{C}(p)-C^{*}$-algebra with spectrum a point, then $A \otimes(\mathbb{K}, \tau)$ is outer equivalent to $(\mathbb{K}, \tau)$ since all automorphisms of $\mathbb{K}$ are given by a conjugation with a unitary. Here, $(\mathbb{K}, \tau)$ denotes the compact operators on $l^{2}(\mathbb{N})$ equipped with the trivial action. Hence $A$ is in $\langle\langle\{\mathbb{C}, \mathcal{C} \mathbf{C}(p)\}\rangle\rangle$. If $A$ is a continuous trace $\mathbf{C}(p)-C^{*}$-algebra with spectrum $\mathbf{C}(p)$, then there are automorphisms $\alpha_{g} \in \operatorname{Aut}(\mathbb{K}), g \in \mathbf{C}(p)$ such that $A \otimes$ $(\mathbb{K}, \tau)$ is isomorphic to

$$
\left(\oplus_{G} \mathbb{K}, \alpha\right), \quad \alpha\left(a_{0}, \ldots, a_{p-1}\right)=\left(\alpha_{[p-1]}\left(a_{p-1}\right), \alpha_{[0]}\left(a_{0}\right), \alpha_{[1]}\left(a_{1}\right), \ldots, \alpha_{[p-2]}\left(a_{p-2}\right)\right) .
$$

Hence $A \otimes(\mathbb{K}, \tau)$ is outer equivalent to $\mathcal{C} \mathbf{C}(p) \otimes(\mathbb{K}, \tau)$. 


\section{Definition of $\mathbf{E K}^{\mathrm{C}(p)}$}

Let $u$ be the unital embedding of $(\mathbb{C}, \tau)$ into $(\mathcal{C} \mathbf{C}(p), \lambda)$, where $\lambda$ denotes the action of $\mathbf{C}(p)$ on $\mathcal{C} \mathbf{C}(p)$ by left translation.

$$
C_{u}=\{a \in \mathcal{C}([0,1), \mathcal{C} \mathbf{C}(p)) \mid a(0) \in \operatorname{Im}(u)\}
$$

is the mapping cone of $u$.

Definition 10.1. Let $\mathcal{A}:=\left\{\mathbb{C}, \mathcal{C} \mathbf{C}(p), C_{u}\right\}$ and

$$
\mathcal{R}:=\mathcal{R}_{\mathcal{A}}=\mathrm{KK}_{*}^{\mathbf{C}(p)}\left(\mathbb{C} \oplus \mathcal{C} \mathbf{C}(p) \oplus C_{u}, \mathbb{C} \oplus \mathcal{C} \mathbf{C}(p) \oplus C_{u}\right),
$$

define

$$
\mathrm{EK}^{\mathbf{C}(p)}:=F_{\mathcal{A}}: \mathfrak{K} \mathfrak{K} \mathbf{C}(p) \rightarrow \mathfrak{M o d}(\mathcal{R})_{\mathrm{c}}^{\mathbb{Z} / 2}
$$

We will calculate $\mathcal{R}$ in terms of generators and relations in Section 11.

Let $B$ be a $\mathbf{C}(p)-C^{*}$-algebra. Note that there is an isomorphism

$$
\mathrm{EK}^{\mathbf{C}(p)}(B) \cong \mathrm{KK}_{*}^{\mathbf{C}(p)}(\mathbb{C}, B) \oplus \mathrm{KK}_{*}^{\mathbf{C}(p)}(\mathcal{C} \mathbf{C}(p), B) \oplus \mathrm{KK}_{*}^{\mathbf{C}(p)}\left(C_{u}, B\right),
$$

which is natural in $B$.

$\mathrm{KK}_{*}^{\mathbf{C}(p)}(\mathbb{C}, B)$ is isomorphic to the $\mathbf{C}(p)$-equivariant K-theory group of $B$ [33]. By the Green-Julg Theorem, there is an isomorphism of abelian groups

$$
\mathrm{KK}_{*}^{\mathbf{C}(p)}(\mathbb{C}, B) \cong \mathrm{K}_{*}(\mathbf{C}(p) \ltimes B) .
$$

Since $\mathcal{C} \mathbf{C}(p) \cong C^{*} \widehat{\mathbf{C}(p)}=C^{*} \mathbf{C}(p)$ (Baaj-Skandalis Duality) the Green-Julg Theorem and Takesaki-Takai duality give an isomorphism of abelian groups

$$
\mathrm{KK}_{*}^{\mathbf{C}(p)}(\mathcal{C} \mathbf{C}(p), B) \cong \mathrm{K}_{*}(B) .
$$

If $p=2$, then the third summand can be interpreted in terms of K-theory of graded $C^{*}$ algebras: Recall that in [23], Kasparov defines KK-theory for $\mathbb{Z} / 2$-graded $C^{*}$-algebras. If $B_{1}$ and $B_{2}$ are $\mathbb{Z} / 2$-graded $C^{*}$-algebras, let us denote the corresponding KK-group in the sense of $[23]$ by $\widehat{\mathrm{KK}}_{*}\left(B_{1}, B_{2}\right)$. Recall that there is also a graded (minimal) tensor product $B_{1} \widehat{\otimes} B_{2}$ with tensor unit $\mathbb{C}$ ( $\mathbb{C}$ is of course trivially graded). We could also define graded K-theory by

$$
\widehat{\mathrm{K}}_{*}\left(B_{1}\right):=\widehat{\mathrm{KK}}_{*}\left(\mathbb{C}, B_{1}\right) .
$$

This agrees with the elementary definition of K-theory for graded Banach algebras as defined for example in [46], for a proof of this fact see [15] 4.5. Of course, every $\mathbb{Z} / 2$ $C^{*}$-algebra $B$ gives rise to a $\mathbb{Z} / 2$-graded $C^{*}$-algebra $\widehat{B}$, where $\widehat{B}=B$ as a $C^{*}$-algebra and the grading involution on $\widehat{B}$ is given by action of the generator of $\mathbb{Z} / 2$. This gives an isomorphism of the categories of (separable) $\mathbb{Z} / 2$-graded $C^{*}$-algebras and (separable) $\mathbb{Z} / 2-C^{*}$-algebras. Let us denote the inverse of $\widehat{\left(\_\right)}$by $\gamma$. In [15], U. Haag studies the relation between KK-theory of graded $C^{*}$-algebras and $\mathbb{Z} / 2$-equivariant KK-theory. Proposition 3.8 in [15] can be rephrased as follows: 
Proposition 10.2. Let $A$ and $B$ be separable $\mathbb{Z} / 2$-algebras. There is a isomorphism

$$
\widehat{\mathrm{KK}}_{*}(\widehat{A}, \widehat{B}) \cong \mathrm{KK}_{*}^{\mathbb{Z} / 2}\left(\gamma\left(\widehat{C_{u}} \widehat{\otimes} \widehat{A}\right), B\right),
$$

which is natural with respect to $\mathbb{Z} / 2$-equivariant $*$-morphisms.

This shows that

$$
\mathrm{KK}_{*}^{\mathbb{Z} / 2}\left(C_{u}, B\right) \cong \widehat{\mathrm{K}}_{*}(\widehat{B})
$$

i.e., that, as an abelian group, the third summand is isomorphic to the graded K-theory of $\widehat{B}$.

Theorem 10.3. $\mathrm{EK}^{\mathrm{C}(p)}$ has the following properties:

(1) $\mathrm{EK}^{\mathbf{C}(p)}$ is exact, i.e., if $B_{1} \longmapsto B_{2} \rightarrow B_{3}$ is a (not necessarily admissible) extension of $G$-C $C^{*}$-algebras, then there is a a natural 6 -term exact sequence

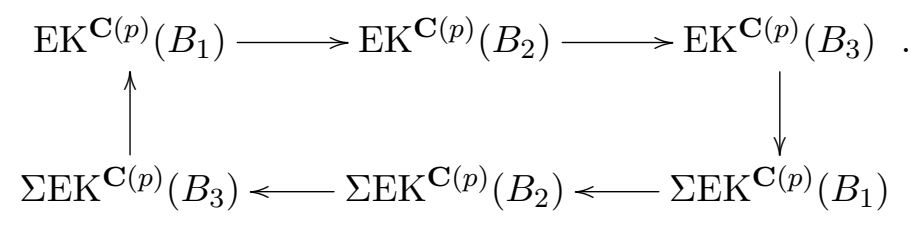

(2) $\mathrm{EK}^{\mathbf{C}(p)}$ commutes with countable direct limits, i.e., if $\left(A_{n}, \alpha_{n}^{m}\right)$ is a countable inductive system of $G$ - $C^{*}$-algebras, then the canonical map

$$
\underset{\lim }{\longrightarrow}\left(\operatorname{EK}^{\mathbf{C}(p)}\left(A_{n}\right), \mathrm{EK}^{\mathbf{C}(p)}\left(\alpha_{n}^{m}\right)\right) \rightarrow \operatorname{EK}^{\mathbf{C}(p)}\left(A_{\infty}\right)
$$

is an isomorphism.

(3) $\mathrm{EK}^{\mathbf{C}(p)}$ has a Thom-isomorphism, i.e., if $B$ is a $\mathbf{C}(p)$-C $C^{*}$-algebra with a commuting $\mathbb{R}$-action, then there is a natural isomorphism

$$
\mathrm{EK}^{\mathbf{C}(p)}(S B) \cong \mathrm{EK}^{\mathbf{C}(p)}(\mathbb{R} \ltimes B) .
$$

(4) $\mathrm{EK}^{\mathbf{C}(p)}$ has a Pimsner-Voiculescu exact sequence, i.e., if $B$ is a $\mathbf{C}(p)-C^{*}$-algebra with a commuting $\mathbb{Z}$-action, then there is a natural 6 -term exact sequence

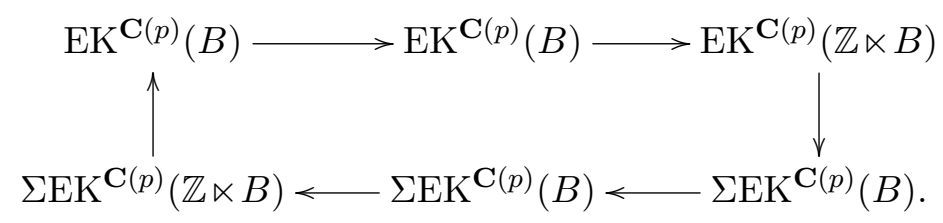

Proof. (1), (3) and (4) are covered by the results of the last section. To prove (2), first note that $\mathrm{K}_{*} \cong \mathrm{KK}_{*}(\mathbb{C}, \ldots)$ commutes with direct limits. Using universal properties, it is also not hard to see that

$$
\mathbf{C}(p) \ltimes A_{\infty} \cong \lim _{\longrightarrow}\left(\mathbf{C}(p) \ltimes A_{n}, \mathbf{C}(p) \ltimes \alpha_{n}^{m}\right) .
$$


Hence $\mathrm{KK}_{*}^{\mathbf{C}(p)}(\mathbb{C}, \ldots)$ commutes with direct limits by the Green-Julg Theorem. Since by Baaj-Skandalis Duality

$$
\mathrm{KK}_{*}^{\mathbf{C}(p)}(\mathcal{C} \mathbf{C}(p), A) \cong \mathrm{KK}_{*}^{\mathbf{C}(p)}(\mathbf{C}(p) \ltimes \mathcal{C} \mathbf{C}(p), \mathbf{C}(p) \ltimes A) \cong \mathrm{KK}_{*}^{\mathbf{C}(p)}(\mathbb{C}, \mathbf{C}(p) \ltimes A),
$$

$\mathrm{KK}_{*}^{\mathbf{C}(p)}(\mathcal{C} \mathbf{C}(p), \ldots)$ also commutes with direct limits. Since $C_{u}$ is the mapping cone of the unital embedding of $\mathbb{C}$ into $\mathcal{C} \mathbf{C}(p)$, the Five Lemma shows that $\operatorname{KK}_{*}^{\mathbf{C}(p)}(\mathcal{C} \mathbf{C}(p), \ldots)$ commutes with direct limits as well.

Theorem 7.1 specializes to

Theorem 10.4. Let $A$ be a separable $\mathbf{C}(p)-C^{*}$-algebra such that

(1) $A \in \mathfrak{B}^{\mathbf{C}(p)}$,

(2) $\operatorname{EK}^{\mathbf{C}(p)}(A)$ has a projective resolution of length 1 in $\mathfrak{M o d}(\mathcal{R})_{\mathrm{c}}^{\mathbb{Z} / 2}$.

Then, for all separable $\mathbf{C}(p)-C^{*}$-algebras $B$, there is a natural short exact sequence

$$
\operatorname{Ext}_{\mathcal{R}}^{1}\left(\operatorname{EK}^{\mathbf{C}(p)}(S A), \operatorname{EK}^{\mathbf{C}(p)}(B)\right) \longmapsto \operatorname{KK}_{*}^{\mathbf{C}(p)}(A, B) \rightarrow \operatorname{Hom}_{\mathcal{R}}\left(\operatorname{EK}^{\mathbf{C}(p)}(A), \operatorname{EK}^{\mathbf{C}(p)}(B)\right) .
$$

We have just seen that every type I $G$ - $C^{*}$-algebra is in $\mathfrak{B}^{\mathbf{C}(p)}$. In Section 12 we will show that condition (2) is always fulfilled. This will prove Theorem 1.1 from the introduction.

\section{Computation of $\mathcal{R}$}

The aim of this section is to compute $\mathcal{R}$. Recall that $\mathcal{R}$ is the category ring of the full subcategory of $\mathfrak{K}_{\mathfrak{K}_{*}}^{\mathbf{C}(p)}$ with objects $\left\{\mathbb{C}, \mathcal{C} \mathbf{C}(p), C_{u}\right\}$.

First we will set up some notation and give explicit formulas for the two main tools which are used to compute $\mathcal{R}$, namely the Green-Julg Theorem and Baaj-Skandalis duality. Then we will start with computing a subring of $\mathcal{R}$, the category ring of $\mathfrak{K}_{\mathfrak{K}_{*}}^{\mathbf{C}(p)}$ restricted to $\{\mathbb{C}, \mathcal{C} \mathbf{C}(p)\}$. The third step is to show that besides the obvious exact triangle coming from the definition of $C_{u}$ as a mapping cone, there is another exact triangle in the opposite direction. Finally, we will include $C_{u}$ in our calculation and determine $\mathcal{R}$ in terms of generators and relations.

\subsection{Preparation}

Let $\mu$ be the Haar measure on $\mathbf{C}(p)$, normalized such that the total mass is $p^{1 / 2}$. Let us fix the isomorphism

$$
\chi: \mathbf{C}(p) \rightarrow \widehat{\mathbf{C}(p)}, \quad \chi_{k}(l)=\mathrm{e}^{2 \pi \mathrm{i} k l / p} .
$$

Fourier transformation gives an equivariant $*$-isomorphism

$$
\mathcal{F}:(\mathcal{C} \mathbf{C}(p), \lambda) \rightarrow\left(C^{*} \mathbf{C}(p), \widehat{\tau}\right), \quad \mathcal{F}(f)(k)=\int_{\mathbf{C}(p)} \chi_{k}(l) f(l) \mathrm{d} \mu(l),
$$


where $\lambda$ is the action by left translation and $\widehat{\tau}$ is the dual of the trivial action.

Let $(A, \alpha)$ be a $\mathbf{C}(p)-C^{*}$-algebra. Let $V$ be a vector space. We will write $A={ }_{\text {vs }} V$ $\left(E={ }_{\mathrm{vs}} V\right)$ if a $C^{*}$-algebra $A$ (Kasparov module $E$ ) is equal to $V$ as a vector space. We have $\mathbf{C}(p) \ltimes{ }_{\alpha} A={ }_{\mathrm{vs}} \mathcal{C}(\mathbf{C}(p), A)$. Since $\mathbf{C}(p)$ is abelian, there is a dual action $\widehat{\alpha}$ of $\widehat{\mathbf{C}(p)} \cong \mathbf{C}(p)$ on $\mathbf{C}(p) \ltimes A$ given by $\widehat{\alpha}_{l}(f)(k):=\chi_{l}(k) f(k)$. By the universal property of $\mathrm{KK}^{\mathbf{C}(p)}$ ([26], Theorem 50) we obtain an endofunctor

$$
\mathbf{C}(p) \ltimes\left(\_\right): \mathfrak{K}_{\mathfrak{K}} \mathbf{C}(p) \rightarrow \mathfrak{K}_{\mathfrak{K}} \mathbf{C}(p) .
$$

\subsection{Baaj-Skandalis Duality}

In [1], S. Baaj and G. Skandalis examine $G \ltimes\left(\_\right)$for a general Hausdorff topological group $G$. We can derive the following explicit description of $\mathbf{C}(p) \ltimes\left(\_\right)$from [1] 6.6 and 6.17:

Let $x=[(\mathcal{E}, T, \gamma)] \in \mathrm{KK}^{\mathbf{C}(p)}(A, B)$. Then $\mathbf{C}(p) \ltimes x$ is represented by $(\mathbf{C}(p) \ltimes \mathcal{E}, \widehat{T}, \widehat{\gamma})$, where $\mathbf{C}(p) \ltimes \mathcal{E}={ }_{\text {vs }} \mathcal{C}(\mathbf{C}(p), \mathcal{E})$ and the expressions for inner product, left and right multiplication specialize to:

$$
(a \xi)(k)=\int_{\mathbf{C}(p)} a(l) \gamma_{l}(\xi(k-l)) \mathrm{d} \mu(l),(\xi b)(k)=\int_{\mathbf{C}(p)} \xi(l) \beta_{l}(b(k-l)) \mathrm{d} \mu(l)
$$

and

$$
\langle\xi, \eta\rangle(k)=\int_{\mathbf{C}(p)} \beta_{-l}(\langle\xi(l), \eta(k+l)\rangle) \mathrm{d} \mu(l) .
$$

for $\xi, \eta \in \mathbf{C}(p) \ltimes \mathcal{E}, b \in \mathbf{C}(p) \ltimes B$ and $a \in \mathbf{C}(p) \ltimes A$. The operator $\widehat{T}$ is given by $(\widehat{T}(\xi))(k)=T(\xi(k))$ and the action $\widehat{\gamma}$ by $\widehat{\gamma}_{l}(\xi)(k)=\chi_{l}(k) \xi(k)$.

Applying the crossed product twice yields a $\mathbf{C}(p)$ - $C^{*}$-algebra $\left(\mathbf{C}(p) \ltimes_{\widehat{\alpha}} \mathbf{C}(p) \ltimes_{\alpha} A, \widehat{\widehat{\alpha}}\right)$, which is naturally $\mathrm{KK}^{\mathbf{C}(p)}$-equivalent to $A$ by the Takai Duality Theorem [43]. Thus $\mathbf{C}(p) \ltimes\left(\_\right)$is an autofunctor of $\mathfrak{K} \mathfrak{K}^{\mathbf{C}(p)}$ which is involutive up to a natural isomorphism

$$
T: \mathbf{C}(p) \ltimes \mathbf{C}(p) \ltimes\left(\_\right) \Rightarrow \operatorname{id}_{\mathfrak{K} \mathfrak{K}} \mathbf{C}(p) .
$$

This observation will be used to simplify the computation of $\mathcal{R}$. It will be useful to explicitly describe the natural transformation $T$.

Let $\left(\mathbb{K}\left(L^{2}(\mathbf{C}(p), A)\right), \operatorname{Ad}_{\rho \otimes \alpha}\right)$ be the compact operators on the Hilbert $A$-module $L^{2}(\mathbf{C}(p), A)$ equipped with the adjoint action, where $\mathbf{C}(p)$ acts on $L^{2}(\mathbf{C}(p), A)$ via

$$
(\rho \otimes \alpha)_{l}(f)(k)=\alpha_{l}(f(k+l)), f \in L^{2}(\mathbf{C}(p), A) .
$$

Lemma 11.1. There is an equivariant $*$-isomorphism

$$
\Phi_{A}:\left(\mathbf{C}(p) \ltimes_{\widehat{\alpha}} \mathbf{C}(p) \ltimes_{\alpha} A, \widehat{\widehat{\alpha}}\right) \rightarrow\left(\mathbb{K}\left(L^{2}(\mathbf{C}(p), A)\right), \operatorname{Ad}_{\rho \otimes \alpha}\right),
$$

which is given by

$$
\Phi_{A}(F)(f)(r)=\int_{\mathbf{C}(p)} \int_{\mathbf{C}(p)} \alpha_{r}^{-1}(F(t, s)) \chi_{t}(s-r) f(r-s) \mathrm{d} \mu(t) \mathrm{d} \mu(s)
$$

for $F \in \mathbf{C}(p) \ltimes_{\widehat{\alpha}} \mathbf{C}(p) \ltimes_{\alpha} A={ }_{\mathrm{vs}} \mathcal{C}(\mathbf{C}(p) \times \mathbf{C}(p), A)$ and $f \in L^{2}(\mathbf{C}(p), A)$. 
Proof. This is a standard result, see for example [48], Theorem 7.1.

Let us denote by $E_{A} \in \mathrm{KK}^{\mathbf{C}(p)}\left(\mathbb{K}\left(L^{2}(\mathbf{C}(p), A)\right), A\right)$ the KK-element which is given by the Morita-Rieffel imprimitivity bimodule $\left(L^{2}(\mathbf{C}(p), A), \rho \otimes \alpha\right)$. Then the natural transformation

$$
T: \mathbf{C}(p) \ltimes \mathbf{C}(p) \ltimes\left(\_\right) \Rightarrow \mathrm{id}
$$

is given by the $\mathrm{KK}^{\mathbf{C}(p)}$ equivalence $T_{A}=\left[\Phi_{A}\right] \cdot E_{A}$. That $T$ is indeed a natural transformation, i.e., that for $\mathbf{C}(p)-C^{*}$-algebras $A$ and $B$ and $x \in \operatorname{KK}^{\mathbf{C}(p)}(A, B)$,

$$
\mathbf{C}(p) \ltimes \mathbf{C}(p) \ltimes x \cdot T_{B}=T_{A} \cdot x
$$

follows from [1], Théorème 6.20 .

Lemma 11.2. Let $A$ be a $\mathbf{C}(p)-C^{*}$-algebra, then

$$
\mathbf{C}(p) \ltimes T_{A}=T_{\mathbf{C}(p) \ltimes A} \text { in } \mathrm{KK}^{\mathbf{C}(p)}(\mathbf{C}(p) \ltimes \mathbf{C}(p) \ltimes \mathbf{C}(p) \ltimes A, \mathbf{C}(p) \ltimes A) .
$$

Proof. $\mathbf{C}(p) \ltimes T_{A}=\left[\left(\mathbf{C}(p) \ltimes L^{2}(\mathbf{C}(p), A)\right)\right]$ and $T_{\mathbf{C}(p) \ltimes A}=\left[\left(L^{2}(\mathbf{C}(p),(\mathbf{C}(p) \ltimes A))\right)\right]$ with left-module structure given by $\mathbf{C}(p) \ltimes \phi_{A}$ and $\phi_{\mathbf{C}(p) \ltimes A}$. Note that both $\mathbf{C}(p) \ltimes L^{2}(\mathbf{C}(p), A)$ and $L^{2}(\mathbf{C}(p),(\mathbf{C}(p) \ltimes A))$ are equal to $\mathcal{C}(\mathbf{C}(p) \times \mathbf{C}(p), A)$ as vector spaces.

$$
\Psi: \mathbf{C}(p) \ltimes L^{2}(\mathbf{C}(p), A) \rightarrow L^{2}(\mathbf{C}(p), \mathbf{C}(p) \ltimes A), \quad \Psi(F)(s, t)=F(t, s),
$$

is clearly bijective. Straightforward calculations show that $\Psi$ intertwines left and right multiplication and the inner products. Therefore, $\Psi$ is an isomorphism of Kasparov $\mathbf{C}(p) \ltimes \mathbf{C}(p) \ltimes \mathbf{C}(p) \ltimes A$ - $\mathbf{C}(p) \ltimes A$-modules.

We will use Baaj-Skandalis duality to construct an honest involution on $\mathfrak{K}_{\mathfrak{K}}{ }^{\mathbf{C}(p)}$ restricted to $\mathbb{C}$ and $\mathcal{C} \mathbf{C}(p)$. This has the advantage of reducing the amount of necessary calculations by a factor of two.

There are $\mathrm{KK}^{\mathbf{C}(p)}$-equivalences

$$
\phi_{0}:=\left[\mathcal{F}^{-1}\right]: \mathbf{C}(p) \ltimes \mathbb{C} \rightarrow \mathcal{C} \mathbf{C}(p) \text { and } \phi_{1}:=[\mathbf{C}(p) \ltimes \mathcal{F}] \cdot T_{\mathbb{C}}: \mathbf{C}(p) \ltimes \mathcal{C} \mathbf{C}(p) \rightarrow \mathbb{C}
$$

For notational reasons, let us set $A_{0}:=\mathbb{C}$ and $A_{1}:=\mathcal{C} \mathbf{C}(p)$.

Definition 11.3. Define an autofunctor $\mathcal{I}$ on objects by $\mathcal{I}\left(A_{i}\right)=A_{i+1}$ and on morphisms by

$$
\mathcal{I}(x)=\phi_{i}^{-1} \cdot(\mathbf{C}(p) \ltimes x) \cdot \phi_{j} \text { for } x \in \mathrm{KK}_{*}^{\mathbf{C}(p)}\left(A_{i}, A_{j}\right)
$$

for $i, j \in\{0,1\}$ (addition is taken modulo 2 ).

Lemma 11.4. $\mathcal{I}$ is an involution.

Proof. Since $\mathcal{I}^{2}(x)=\phi_{i+1}^{-1} \cdot\left(\mathbf{C}(p) \ltimes \phi_{i}^{-1}\right) \cdot(\mathbf{C}(p) \ltimes \mathbf{C}(p) \ltimes x) \cdot\left(\mathbf{C}(p) \ltimes \phi_{j}\right) \cdot \phi_{j+1}$, it is sufficient to show that $\left(\mathbf{C}(p) \ltimes \phi_{i}\right) \cdot \phi_{i+1}=T_{A_{i}}$ for $i \in\{0,1\}$. For $i=0$, the statement is obvious and for $i=1$, it follows from Lemma 11.2 and naturality of $T_{A}$. 


\subsection{The Green-Julg Theorem}

Another useful tool is the Green-Julg Theorem [21]. A KK-theoretic formulation appears in $[12]$.

Theorem 11.5 (Green-Julg Theorem). Let $G$ be a compact group and $A$ a $G-C^{*}$ algebra. Then there is a natural isomorphism

$$
\nu: \mathrm{KK}^{G}(\mathbb{C}, A) \rightarrow \mathrm{KK}(\mathbb{C}, G \ltimes A) .
$$

Let us give explicit formulas for the present case $G=\mathbf{C}(p)$ : Let $A$ be a $\mathbf{C}(p)$ $C^{*}$-algebra and $x=\left[\left(\mathcal{E}_{A}, T, \gamma\right)\right]$ an element in $\operatorname{KK}^{\mathbf{C}(p)}(\mathbb{C}, A)$. Then $\nu(x)$ is given by $\left[\left(\mathcal{E}_{\mathbf{C}(p) \ltimes A}, T\right)\right]$. Here, $\mathcal{E}_{\mathbf{C}(p) \ltimes A}$ is equal to $\mathcal{E}_{A}$ as a vector space and carries the operations

$$
\xi \cdot f=\int_{\mathbf{C}(p)} \gamma_{l}(\xi) f(-l) \mathrm{d} \mu(l) \text { and }\langle\xi, \eta\rangle_{\mathbf{C}(p) \ltimes A}(l)=\left\langle\xi, \gamma_{l}(\eta)\right\rangle_{A}
$$

for $\xi, \eta \in \mathcal{E}_{A}$ and $f \in \mathbf{C}(p) \ltimes A$. The operator $T$ remains unchanged.

\subsection{A Subring of $\mathcal{R}$}

Our first aim is to calculate the category ring of $\mathfrak{K}_{\mathfrak{K}_{*}} \mathbf{C}(p)$ restricted to $\mathbb{C}$ and $\mathcal{C} \mathbf{C}(p)$.

Definition 11.6. Let $e$ denote the class of 1 in $\mathbf{C}(p)$ and define ${ }_{0} t_{0}:=\left[\chi_{e}\right] \in \operatorname{KK}^{\mathbf{C}(p)}(\mathbb{C}, \mathbb{C})$, in other words, ${ }_{0} t_{0}$ is given by the class of the character $\chi_{e} \in \widehat{\mathbf{C}(p)}$.

A representing Kasparov triple of ${ }_{0} t_{0}$ is given by $\left(\mathbb{C}, 0, \chi_{e}\right)$.

\section{Lemma 11.7.}

$$
\mathbb{Z}[t] /\left\langle t^{p}-1\right\rangle \cong \mathrm{KK}_{0}^{\mathbf{C}(p)}(\mathbb{C}, \mathbb{C}), \quad t \mapsto{ }_{0} t_{0}
$$

is an isomorphism of rings and $\mathrm{KK}_{1}^{\mathbf{C}(p)}(\mathbb{C}, \mathbb{C}) \cong\{0\}$.

Proof. This is a standard result: For a compact group $G, \mathrm{KK}_{*}^{G}(\mathbb{C}, \mathbb{C})$ is canonically isomorphic to the complex representation ring concentrated in degree 0 , see for example [23], the remark following Corollary 2.15.

Definition 11.8. Let ${ }_{1} s_{1}:=\mathcal{I}\left({ }_{0} t_{0}\right) \in \mathrm{KK}_{0}^{\mathrm{C}(p)}(\mathcal{C} \mathbf{C}(p), \mathcal{C} \mathbf{C}(p))$.

\section{Corollary 11.9.}

$$
\mathbb{Z}[s] /\left\langle s^{p}-1\right\rangle \cong \mathrm{KK}_{0}^{\mathbf{C}(p)}(\mathcal{C} \mathbf{C}(p), \mathcal{C} \mathbf{C}(p)), \quad s \mapsto{ }_{1} s_{1}
$$

is an isomorphism of rings and $\mathrm{KK}_{1}^{\mathbf{C}(p)}(\mathcal{C} \mathbf{C}(p), \mathcal{C} \mathbf{C}(p)) \cong\{0\}$.

Note that

$$
\left[\mathcal{F}^{-1}\right] \cdot{ }_{1} s_{1} \cdot[\mathcal{F}]=\mathbf{C}(p) \ltimes\left[\chi_{e}\right]=\left[\widehat{\tau}_{e}\right]=\left[\mathcal{F}^{-1}\right] \cdot\left[\lambda_{e}\right] \cdot[\mathcal{F}]
$$

by equivariance of $\mathcal{F}$. This shows that ${ }_{1} s_{1}$ is given by translation by the generator of the action on $\mathcal{C} \mathbf{C}(p)$. 
Definition 11.10. Let $u: \mathbb{C} \rightarrow \mathcal{C C}(p)$ be the unital embedding and set

$$
{ }_{0} \alpha_{1}:=[u] \in \mathrm{KK}^{\mathbf{C}(p)}(\mathbb{C}, \mathcal{C} \mathbf{C}(p)) .
$$

Lemma 11.11. $\mathrm{KK}_{*}^{\mathbf{C}(p)}(\mathbb{C}, \mathcal{C} \mathbf{C}(p)) \cong \mathbb{Z}[0]$ with generator ${ }_{0} \alpha_{1}$.

Proof. The Green-Julg isomorphism $\nu$ and the equivariant $*$-isomorphism $\Phi_{\mathbb{C}} \circ(\mathbf{C}(p) \ltimes \mathcal{F})$ induce an isomorphism

$$
\mathrm{KK}_{*}^{\mathbf{C}(p)}(\mathbb{C}, \mathcal{C} \mathbf{C}(p)) \cong \mathrm{KK}_{*}(\mathbb{C}, \mathbf{C}(p) \ltimes \mathcal{C} \mathbf{C}(p)) \cong \mathrm{KK}_{*}\left(\mathbb{C}, \mathbb{K}\left(L^{2}(\mathbf{C}(p))\right)\right) \cong \mathbb{Z}[0] .
$$

Let $\nu([u])$ be represented by $[(\mathcal{E}, 1,0)]$, then $\mathcal{E}={ }_{\mathrm{vs}} \mathcal{C} \mathbf{C}(p)$. Define a projection $P \in$ $\mathbf{C}(p) \ltimes \mathcal{C} \mathbf{C}(p)$ by $P(k, l):=c_{p} \forall k, l \in \mathbf{C}(p)$. A calculation using the formulas following Theorem 11.5 shows that

$$
\Psi: E \rightarrow \operatorname{im} P, \quad \Psi(f)(k, l)=f(l-k),
$$

is an isomorphism of Hilbert modules. Another calculation using the expression of $\Phi_{\mathbb{C}}$ given in Lemma 11.1 shows that $\Phi_{\mathbb{C}} \circ \mathbf{C}(p) \ltimes \mathcal{F}$ maps $P$ to the 1-dimensional projection $\left|\delta_{0}\right\rangle\left\langle\delta_{0}\right| \in \mathbb{K}\left(L^{2}(\mathbf{C}(p))\right)$. This shows the claim.

Definition 11.12. Let ${ }_{1} \alpha_{0}:=\mathcal{I}\left({ }_{0} \alpha_{1}\right) \in \operatorname{KK}_{0}^{\mathbf{C}(p)}(\mathcal{C C}(p), \mathbb{C})$.

Corollary 11.13. $\mathrm{KK}_{*}^{\mathbf{C}(p)}(\mathcal{C} \mathbf{C}(p), \mathbb{C}) \cong \mathbb{Z}[0]$ with generator ${ }_{1} \alpha_{0}$.

Recall that $E_{\mathbb{C}}$ denotes the imprimitivity bimodule between $\mathbb{K}\left(L^{2}(\mathbf{C}(p))\right)$ and $\mathbb{C}$. By definition ${ }_{1} \alpha_{0}=[\mathcal{F}] \cdot[\mathbf{C}(p) \ltimes u] \cdot[\mathbf{C}(p) \ltimes \mathcal{F}] \cdot T_{\mathbb{C}}=\left[\Phi_{\mathbb{C}} \circ(\mathbf{C}(p) \ltimes(\mathcal{F} \circ u)) \circ \mathcal{F}\right] \cdot E_{\mathbb{C}} \cdot \mathbf{A}$ computation shows that the $*$-homomorphism $\Phi_{\mathbb{C}} \circ(\mathbf{C}(p) \ltimes(\mathcal{F} \circ u)) \circ \mathcal{F}$ is equal to

$$
m: \mathcal{C} \mathbf{C}(p) \rightarrow \mathbb{K}\left(L^{2}(\mathbf{C}(p))\right), \quad m(f)(h)=f \cdot h .
$$

Hence ${ }_{1} \alpha_{0}=[m] \cdot E_{\mathbb{C}}$.

Definition 11.14. Let $R$ be a unital ring and $t \in R$ with $t^{p}=1$. Define $N(t):=\sum_{k=0}^{p-1} t^{k}$.

Lemma 11.15.

$$
\begin{aligned}
& { }_{0} \alpha_{1} \cdot{ }_{1} \alpha_{0}=N\left({ }_{0} t_{0}\right) \in \mathrm{KK}^{\mathbf{C}(p)}(\mathbb{C}, \mathbb{C}) . \\
& { }_{1} \alpha_{0} \cdot{ }_{0} \alpha_{1}=N\left({ }_{1} s_{1}\right) \in \mathrm{KK}^{\mathbf{C}(p)}(\mathcal{C} \mathbf{C}(p), \mathcal{C} \mathbf{C}(p)) .
\end{aligned}
$$

Proof. Since ${ }_{0} \alpha_{1} \cdot{ }_{1} \alpha_{0}=[m \circ u] \cdot\left[E_{\mathbb{C}}\right]$, the first product is equal to the class of the representation $\left[\left(L^{2}(\mathbf{C}(p)), \lambda\right)\right] \in \mathrm{KK}_{*}^{\mathbf{C}(p)}(\mathbb{C}, \mathbb{C})$. The Peter-Weyl theorem implies ${ }_{0} \alpha_{1}$. ${ }_{1} \alpha_{0}=\left[\left(L^{2}(\mathbf{C}(p)), \lambda\right)\right]=\sum_{k \in \mathbf{C}(p)}\left[\chi_{k}\right]=N\left({ }_{0} t_{0}\right)$. The second equality follows by applying $\mathcal{I}$.

\section{Lemma 11.16.}

$$
\begin{aligned}
& { }_{0} t_{0} \cdot{ }_{0} \alpha_{1}={ }_{0} \alpha_{1} \cdot{ }_{1} s_{1}={ }_{0} \alpha_{1} \in \mathrm{KK}^{\mathbf{C}(p)}(\mathbb{C}, \mathcal{C} \mathbf{C}(p)) . \\
& { }_{1} s_{1} \cdot{ }_{1} \alpha_{0}={ }_{1} \alpha_{0} \cdot{ }_{0} t_{0}={ }_{1} \alpha_{0} \in \mathrm{KK}^{\mathbf{C}(p)}(\mathcal{C} \mathbf{C}(p), \mathbb{C}) .
\end{aligned}
$$


Proof. ${ }_{0} t_{0} \cdot{ }_{0} \alpha_{1}={ }_{0} \alpha_{1} \cdot\left[\chi_{e}\right] \otimes_{\mathbb{C}} 1_{\mathcal{C} \mathbf{C}(p)}$ and $\left[\chi_{e}\right] \otimes_{\mathbb{C}} 1_{\mathcal{C} \mathbf{C}(p)}$ is represented by $\left(\mathcal{C}(\mathbf{C}(p)), 0, \chi_{e} \otimes\right.$ $\lambda)$. Since

$$
(\mathcal{C} \mathbf{C}(p), \lambda) \rightarrow\left(\mathcal{C} \mathbf{C}(p), \chi_{e} \otimes \lambda\right), \quad f \mapsto \chi_{e} f,
$$

is an isomorphism of Kasparov $\mathcal{C} \mathbf{C}(p)-\mathcal{C} \mathbf{C}(p)$ bimodules, we conclude $\left[\chi_{e}\right] \otimes_{\mathbb{C}} 1_{\mathcal{C}(p)}=$ $1_{\mathcal{C} \mathbf{C}(p)}$. Hence ${ }_{0} t_{0} \cdot{ }_{0} \alpha_{1}={ }_{0} \alpha_{1}$. Furthermore, we have ${ }_{0} \alpha_{1} \cdot{ }_{1} s_{1}=[u] \cdot \lambda_{e}=\tau_{e} \cdot[u]=[u]=$ ${ }_{0} \alpha_{1}$. This shows the first equalities. ${ }_{1} s_{1} \cdot{ }_{1} \alpha_{0}={ }_{1} \alpha_{0} \cdot{ }_{0} t_{0}={ }_{1} \alpha_{0}$ follows by applying $\mathcal{I}$.

\subsection{The First Exact Triangle}

Let $\phi$ be the $\mathbf{C}(p)$-action on the complex plane given by rotation by an angle of $2 \pi / p$. Throughout the next two subsections, every $\mathbf{C}(p)$-invariant subset of $\mathbb{C}$ is tacitly assumed to be equipped with the action given by the restriction of $\phi$. Recall that $C_{u}$ is the mapping cone of the unital embedding $u: \mathbb{C} \rightarrow \mathcal{C} \mathbf{C}(p)$. Let

$$
X:=\left\{t \mathrm{e}^{2 \pi \mathrm{i} l / p} \in \mathbb{C} \mid t \in[0,1) l=0, \ldots, p-1\right\} .
$$

We may identify $C_{u}$ with $\mathcal{C}_{0}(X)$ as $\mathbf{C}(p)$ - $C^{*}$-algebras. Evaluation at 0 yields an evaluation morphism

$$
\epsilon_{u}: C_{u} \rightarrow \mathbb{C}
$$

We may identify $S \mathcal{C C}(p)$ with the kernel of $\epsilon_{u}$, this gives an inclusion morphism

$$
\iota_{u}: \operatorname{SCC}(p) \rightarrow C_{u} .
$$

Definition 11.17. Let ${ }_{2} \alpha_{0}:=\left[\epsilon_{u}\right] \in \mathrm{KK}_{0}\left(C_{u}, \mathbb{C}\right)$ and ${ }_{1} \alpha_{2}:=\left[\iota_{u}\right] \in \operatorname{KK}_{1}\left(\mathcal{C C}(p), C_{u}\right)$.

$C_{u}$ sits in the exact triangle

$$
\operatorname{SCC}(p) \stackrel{{ }^{1 \alpha_{2}}}{\longrightarrow} C_{u} \stackrel{{ }^{2} \alpha_{0}}{\longrightarrow} \mathbb{C} \stackrel{{ }^{0 \alpha_{1}}}{\longrightarrow} \mathcal{C} \mathbf{C}(p) .
$$

We will refer to this exact triangle as the first exact triangle.

\subsection{The Second Exact Triangle}

There is another exact triangle, which will be derived by working with a homotopy equivalent model of $C_{u}$. Define a subset of the closed unit ball $D^{1}$ in $\mathbb{C}$ by

$$
Y:=D^{1} \backslash\left\{\mathrm{e}^{2 \pi \mathrm{i} l / p} \mid l=0, \ldots, p-1\right\} .
$$

It is easy to see that $X$ is a $\mathbf{C}(p)$-equivariant deformation retract of $Y$ - there are *-homomorphisms $r: \mathcal{C}_{0}(Y) \rightarrow \mathcal{C}_{0}(X)$ and $i: \mathcal{C}_{0}(X) \rightarrow \mathcal{C}_{0}(Y)$ such that $r \circ i=\operatorname{id}_{\mathcal{C}_{0}(X)}$ and $i \circ r$ is $\mathbf{C}(p)$-equivariantly homotopic to $\operatorname{id}_{\mathcal{C}_{0}(Y)}$. Let

$$
A:=\mathbb{T}^{1} \backslash\left\{\mathrm{e}^{2 \pi \mathrm{i} l / p} \mid l=0, \ldots, p-1\right\} .
$$

$\mathcal{C}_{0}(A)$ is $\mathbf{C}(p)$-equivariantly isomorphic to $S \mathcal{C} \mathbf{C}(p)$ via

$$
\psi: \mathcal{C}_{0}(A) \rightarrow S C \mathbf{C}(p), \quad \psi(f)(t, k)=f\left(\mathrm{e}^{2 \pi \mathrm{i}(k-t) / p}\right), k \in \mathbf{C}(p), t \in(0,1) .
$$


Since $A$ is closed in $Y$, this yields a restriction morphism $q: \mathcal{C}_{0}(Y) \rightarrow S C \mathbf{C}(p)$. Let $U=D^{1} \backslash \mathbb{T}^{1}$ be the complement of $A$ in $Y$. $U$ is $\mathbf{C}(p)$-equivariantly homeomorphic to $\mathbb{C}$. Let $j: \mathcal{C}_{0}(\mathbb{C}) \rightarrow \mathcal{C}(Y)$ be the morphism corresponding to the inclusion of $U$ in $Y$. We obtain an exact triangle

$$
S^{2} \mathcal{C C}(p) \stackrel{\delta}{\longrightarrow} \mathcal{C}_{0}(\mathbb{C}) \stackrel{[j]}{\longrightarrow} \mathcal{C}_{0}(Y) \stackrel{[q]}{\longrightarrow} S \mathcal{C C}(p) .
$$

Since the action $\phi$ on $\mathbb{C}$ comes from the restriction of the action of $S^{1}$ on $\mathbb{C}$, which is spinor, there is a $\mathbf{C}(p)$-equivariant Bott element $\mathcal{B}_{\phi} \in \mathrm{KK}^{\mathbf{C}(p)}\left(\mathbb{C}, \mathcal{C}_{0}(\mathbb{C})\right)$ and a $\mathbf{C}(p)$ equivariant Dirac element $\mathcal{D}_{\phi} \in \mathrm{KK}^{\mathbf{C}(p)}\left(\mathcal{C}_{0}(\mathbb{C}), \mathbb{C}\right)$ such that $\mathcal{B}_{\phi} \cdot \mathcal{D}_{\phi}=1_{\mathbb{C}, \mathbb{C}}$ and $\mathcal{D}_{\phi} \cdot \mathcal{B}_{\phi}=$ $1_{\mathcal{C}_{0}(\mathbb{C})}([22]$ Section 5 , especially Theorem 7$)$.

Lemma 11.18. We have $\mathcal{B}_{\mathcal{C}(\mathbf{C}(p))} \cdot \delta \cdot \mathcal{D}_{\phi}={ }_{1} \alpha_{0} \in \mathrm{KK}_{0}^{\mathbf{C}(p)}(\mathcal{C} \mathbf{C}(p), \mathbb{C})$.

Proof. Since ${ }_{1} \alpha_{0}$ is a generator of $\operatorname{KK}_{0}^{\mathbf{C}(p)}(\mathcal{C} \mathbf{C}(p), \mathbb{C}) \cong \mathbb{Z}$, there is an $m \in \mathbb{Z}$ such that

$$
\mathcal{B}_{\mathcal{C}(\mathbf{C}(p))} \cdot \delta \cdot \mathcal{D}_{\phi}=m \cdot{ }_{1} \alpha_{0} .
$$

We have to show that $m=1$. Let $\vartheta: \mathfrak{K} \mathfrak{K} \mathbf{C}(p) \rightarrow \mathfrak{K} \mathfrak{K}$ denote the forgetful functor. $\vartheta\left(\mathcal{D}_{\phi}\right)=\mathcal{D}$ implies

$$
\vartheta(\delta)=m \mathcal{D}_{\mathcal{C}(\mathbf{C}(p))} \cdot \vartheta\left({ }_{1} \alpha_{0}\right) \cdot \mathcal{B}=m S^{2} \vartheta\left({ }_{1} \alpha_{0}\right) \cdot \mathcal{D} \cdot \mathcal{B}=m S^{2} \vartheta\left({ }_{1} \alpha_{0}\right) .
$$

Of course $\vartheta(\mathcal{C} \mathbf{C}(p)) \cong \oplus_{i=1}^{p} \mathbb{C}$. Let $\iota_{i}$ and $\pi_{i}$ denote the inclusion and projection of the $i$ th summand. Pullback of the extension representing $\vartheta(\delta)$ along $S \iota_{1}$ yields an extension, which is homotopic to

$$
S^{2} \longmapsto S \mathcal{C}_{0}((0,1]) \rightarrow S .
$$

The latter is well known to represent the unit in $\mathrm{KK}\left(S^{2}, S^{2}\right)=\mathrm{KK}_{1}\left(S, S^{2}\right)$ ([6] 19.2). This shows $\left[S^{2} \iota_{1}\right] \cdot \vartheta(\delta)=1_{S^{2}}$. On the other hand $\vartheta\left({ }_{1} \alpha_{0}\right)=\sum_{i}\left[\pi_{i}\right]$, which implies that $\left[S^{2} \iota_{1}\right] \cdot S^{2} \vartheta\left({ }_{1} \alpha_{0}\right)=1_{S^{2}}$. This shows $m=1$.

So far, we have constructed a diagram in $\mathfrak{K} \mathfrak{K}^{\mathbf{C}(p)}$

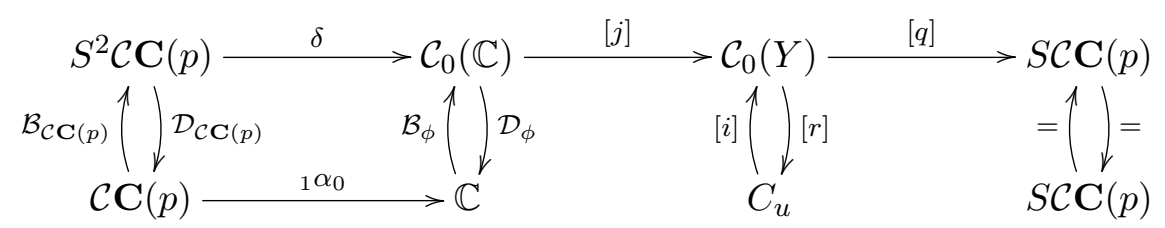

with the following properties

1. the upper row is an exact triangle,

2. each pair of vertical arrows in one column consists of isomorphisms which are mutual inverses,

3. the left square commutes. 
Definition 11.19. Set

$$
{ }_{0} \alpha_{2}:=\mathcal{B}_{\phi} \cdot[j] \cdot[r] \in \mathrm{KK}_{0}\left(\mathbb{C}, C_{u}\right) \text { and }{ }_{2} \alpha_{1}:=S[i] \cdot S[q] \cdot \mathcal{D}_{\mathcal{C}(p)} \in \mathrm{KK}_{1}\left(C_{u}, \mathcal{C} \mathbf{C}(p)\right) .
$$

Remark 11.20. We define ${ }_{2} \alpha_{1}$ as $S[r] \cdot S[q] \cdot \mathcal{D}_{\mathcal{C} \mathbf{C}(p)}$ instead of simply $[r] \cdot[q]$ due to our convention that $\operatorname{KK}_{1}(A, B)=\operatorname{KK}(S A, B)$.

\section{Corollary 11.21.}

$$
S C_{u} \stackrel{{ }_{2} \alpha_{1}}{\longrightarrow} \mathcal{C} \mathbf{C}(p) \stackrel{{ }^{1 \alpha_{0}}}{\longrightarrow} \mathbb{C} \stackrel{{ }_{0} \alpha_{2}}{\longrightarrow} C_{u}
$$

is an exact triangle.

Proof. The triangle in the statement of the corollary is isomorphic to

$$
S \mathcal{C}_{0}(Y) \stackrel{-S[q]}{\longrightarrow} S^{2} \mathcal{C} \mathbf{C}(p) \stackrel{\delta}{\longrightarrow} \mathcal{C}_{0}(\mathbb{C}) \stackrel{[j]}{\longrightarrow} \mathcal{C}_{0}(Y)
$$

We will refer to this exact triangle as the second exact triangle.

Remark 11.22. In the case $p=2$, the second exact triangle already appears in a different setup in [15].

\subsection{Morphisms from and into $C_{u}$}

Proposition 11.23. Let $k \in\{0, \ldots p-1\}$ and $I:=\{0, \ldots p-1\} \backslash\{k\}$. Then

$$
\begin{aligned}
\mathrm{KK}_{*}{ }^{\mathbf{C}(p)}\left(C_{u}, \mathbb{C}\right) & \cong \mathbb{Z}^{p-1}[0] \text { with basis }\left({ }_{2} \alpha_{0} \cdot\left({ }_{0} t_{0}\right)^{i}\right)_{i \in I} . \\
\mathrm{KK}_{*}{ }^{\mathbf{C}(p)}\left(\mathbb{C}, C_{u}\right) & \cong \mathbb{Z}^{p-1}[0] \text { with basis }\left(\left({ }_{0} t_{0}\right)^{i} \cdot{ }_{0} \alpha_{2}\right)_{i \in I} . \\
\mathrm{KK}_{*}{ }^{\mathbf{C}(p)}\left(C_{u}, \mathcal{C} \mathbf{C}(p)\right) & \cong \mathbb{Z}^{p-1}[1] \text { with basis }\left({ }_{2} \alpha_{1} \cdot\left({ }_{1} s_{1}\right)^{i}\right)_{i \in I} \\
\mathrm{KK}_{*}{ }^{\mathbf{C}(p)}\left(\mathcal{C} \mathbf{C}(p), C_{u}\right) & \cong \mathbb{Z}^{p-1}[1] \text { with basis }\left(\left({ }_{1} s_{1}\right)^{i} \cdot{ }_{1} \alpha_{2}\right)_{i \in I} .
\end{aligned}
$$

Proof. Applying $\mathrm{KK}_{0}^{\mathrm{C}(p)}$ (_, $\left.\mathbb{C}\right)$ to the first exact triangle yields a 6-term exact sequence

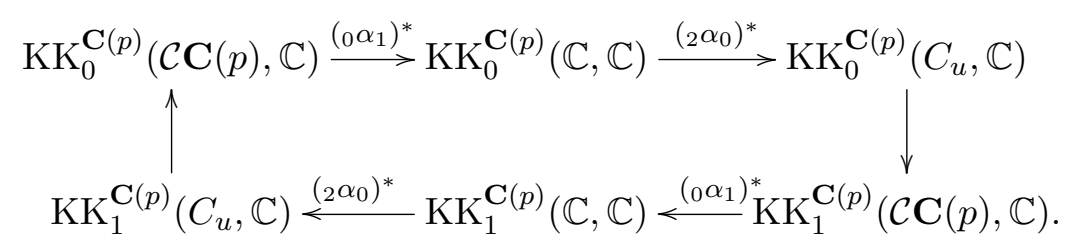

We already know that $\mathrm{KK}_{1}^{\mathbf{C}(p)}(\mathcal{C} \mathbf{C}(p), \mathbb{C})=\{0\}$. Furthermore we know that $\left({ }_{0} \alpha_{1}\right)^{*}$ is one-to-one by Lemma 11.15 and Lemma 11.13. Hence $\operatorname{KK}_{1}^{\mathbf{C}(p)}\left(C_{u}, \mathbb{C}\right)=\{0\}$ and we obtain a short exact sequence

$$
\left.0 \longrightarrow \mathrm{KK}_{0}^{\mathbf{C}(p)}(\mathcal{C} \mathbf{C}(p), \mathbb{C}) \stackrel{\left({ }_{0} \alpha_{1}\right)^{*}}{\longrightarrow} \mathrm{KK}_{0}^{\mathbf{C}(p)}(\mathbb{C}, \mathbb{C}) \stackrel{\left({ }_{2} \alpha_{0}\right)^{*}}{\longrightarrow} \mathrm{KK}_{0}^{\mathbf{C}(p)}\left(C_{u}, \mathbb{C}\right)\right) \longrightarrow 0
$$


We have $\mathrm{KK}_{0}^{\mathbf{C}(p)}(\mathbb{C}, \mathbb{C}) \cong \mathbb{Z}\left[{ }_{0} t_{0}\right] /\left\langle{ }_{0} t_{0}^{p}-1\right\rangle$ with $\operatorname{im}\left({ }_{0} \alpha_{1}\right)^{*}$ being the ideal generated by $N\left({ }_{0} t_{0}\right)$. This shows the first statement. The remaining statements are proven analogously: For the second statement, apply $\operatorname{KK}_{0}^{\mathbf{C}(p)}(\mathbb{C}, \ldots)$ to the second exact triangle, for the third statement apply $\mathrm{KK}_{1}^{\mathbf{C}(p)}(\ldots, \mathcal{C} \mathbf{C}(p))$ to the second exact triangle and for the last statement apply $\mathrm{KK}_{1}^{\mathbf{C}(p)}(\mathcal{C} \mathbf{C}(p), \ldots)$ to the first exact triangle.

Proposition 11.24.

$$
{ }_{0} \alpha_{2} \cdot{ }_{2} \alpha_{0}=1-{ }_{0} t_{0} \in \mathrm{KK}_{0}^{\mathrm{C}(p)}(\mathbb{C}, \mathbb{C})
$$

Proof. By definition,

$$
{ }_{0} \alpha_{2} \cdot{ }_{2} \alpha_{0}=\mathcal{B}_{\phi} \cdot[j] \cdot[r] \cdot\left[\epsilon_{u}\right]=\mathcal{B}_{\phi}\left[\epsilon_{u} \circ r \circ j\right] .
$$

$\epsilon_{u} \circ r \circ j$ is the $*$-homomorphism given by evaluation at 0 :

$$
\epsilon_{u} \circ r \circ j: \mathcal{C}_{0}(\mathbb{C}) \rightarrow \mathbb{C}, \quad f \mapsto f(0) .
$$

Let us describe $\mathcal{B}_{\phi} \in \mathrm{KK}_{0}^{\mathrm{C}(p)}\left(\mathbb{C}, \mathcal{C}_{0}(\mathbb{C})\right)$ in more detail ([22] Section 5): Under the identification $\mathbb{R}^{2} \cong \mathbb{C}$, the action $\phi$ of $\mathbf{C}(p)$ on $\mathbb{R}^{2}$ is given by rotation by the angle $2 \pi / p$. Let $S_{2}=\mathbb{C}^{2}$ be the 2-dimensional complex spinors, graded by $S_{2}^{(0)}=\mathbb{C} \cdot(1,-\mathrm{i})$ and $S_{2}^{(1)}=\mathbb{C} \cdot(1, \mathrm{i})$. Pointwise application of the standard scalar product on $S_{2}$ endows $\mathcal{C}_{0}\left(\mathbb{R}^{2}, S_{2}\right)$ with the structure of a graded Hilbert $\mathcal{C}_{0}(\mathbb{C})$-module. Define an action of $\mathbf{C}(p)$ on $S_{2}$ by

$$
\rho_{k}:=\mathrm{e}^{\pi \mathrm{i} k / p}\left(\begin{array}{cc}
\cos (\pi k / p) & \sin (\pi k / p) \\
-\sin (\pi k / p) & \cos (\pi k / p)
\end{array}\right) .
$$

$\mathbf{C}(p)$ acts on $\mathcal{C}_{0}\left(\mathbb{R}^{2}, S_{2}\right)$ by $\phi \otimes \rho(f)=\rho \circ f \circ \phi^{-1}$. Set

$$
\sigma_{1}:=\left(\begin{array}{cc}
0 & \mathrm{i} \\
\mathrm{i} & 0
\end{array}\right), \quad \sigma_{2}:=\left(\begin{array}{cc}
\mathrm{i} & 0 \\
0 & -\mathrm{i}
\end{array}\right) .
$$

Define an adjoinable operator $\mathrm{F}$ on $\mathcal{C}\left(\mathbb{R}^{2}, S_{2}\right)$ by

$$
F(f)(x, y)=\left(1+x^{2}+y^{2}\right)^{-1 / 2}\left(x \sigma_{1}+y \sigma_{2}\right) f(x, y) .
$$

Then

$$
\mathcal{B}_{\phi}=\left[\left(\mathcal{C}_{0}\left(\mathbb{R}^{2}, S_{2}\right), F, \phi \otimes \rho\right)\right]
$$

Therefore

$$
\mathcal{B}_{\phi} \cdot[j] \cdot[i] \cdot\left[\epsilon_{u}\right]=\left(\epsilon_{u} \circ i \circ j\right)_{*}\left(\mathcal{B}_{\phi}\right)=\left[\left(S_{2}, 0, \rho\right)\right] \in \mathrm{KK}_{0}^{\mathbf{C}(p)}(\mathbb{C}, \mathbb{C}) .
$$

But since $\rho_{k}((1,-\mathrm{i}))=(1,-\mathrm{i})$ and $\rho_{k}((1, \mathrm{i}))=e^{2 \pi \mathrm{i} k / p}(1, \mathrm{i})$, we have

$$
\left[\left(S_{2}, 1,0, \rho\right)\right]=1-{ }_{0} t_{0} \in \mathrm{KK}_{0}^{\mathbf{C}(p)}(\mathbb{C}, \mathbb{C}) .
$$

This proves the proposition. 


\section{Proposition 11.25.}

$$
{ }_{1} \alpha_{2} \cdot{ }_{2} \alpha_{1}=1-{ }_{1} s_{1} \in \mathrm{KK}_{0}^{\mathbf{C}(p)}\left(\mathcal{C}_{0}(\mathbf{C}(p)), \mathcal{C}_{0}(\mathbf{C}(p))\right) .
$$

Proof. By definition, ${ }_{1} \alpha_{2} \cdot{ }_{2} \alpha_{1}=\mathcal{B}_{\mathcal{C} \mathbf{C}(p)} \cdot S\left[\iota_{u}\right] \cdot S[i] \cdot S[q] \cdot \mathcal{D}_{\mathcal{C} \mathbf{C}(p)}$ (the $\mathcal{B}_{\mathcal{C} \mathbf{C}(p)}$ on the left comes from our convention on the composition in $\mathfrak{K}_{\mathfrak{K}_{*}^{G}}^{G}$. It is not hard to see that the equivariant $*$-homomorphism

$$
q \circ i \circ \iota_{u}: S C \mathbf{C}(p) \rightarrow S C \mathbf{C}(p)
$$

is given by

$$
q \circ i \circ \iota_{u}(f)(t, k)= \begin{cases}f(2 t, k) & \text { if } 0<t<\frac{1}{2} \\ 0 & \text { if } t=\frac{1}{2}, \\ f(2-2 t, k-e) & \text { if } \frac{1}{2}<t<1\end{cases}
$$

for all $k \in \mathbf{C}(p)$.

Standard homotopy arguments show that if $A$ and $B$ are $G$ - $C^{*}$-algebras and $\phi_{0}, \phi_{1}: A \rightarrow$ $S B$ are equivariant $*$-homomorphisms, then

$$
\left[\phi_{0}\right]+\left[\phi_{1}\right]=\left[\phi_{01}\right] \in \mathrm{KK}^{G}(A, S B),
$$

where $\phi_{01}$ is the $*$-homomorphism given by

$$
\phi_{01}(a)(t)=\left\{\begin{array}{ll}
\phi_{0}(a)(2 t) & \text { if } 0<t<\frac{1}{2} \\
0 & \text { if } t=\frac{1}{2} \\
\phi_{1}(a)(2 t-1) & \text { if } \frac{1}{2}<t<1
\end{array},\right.
$$

for $a \in A$. Hence we have

$$
\left[q \circ i \circ \iota_{u}\right]=1_{S C \mathbf{C}(p)}+\left[v \otimes_{C} \lambda_{e}\right]
$$

where $v: S \rightarrow S$ is given by $v(f)(t)=f(1-t)$. Using the fact above again we see that $[v]+1_{S}=[\psi]$, where $\psi$ is given by

$$
\psi(f)(t)= \begin{cases}f(2 t) & \text { if } 0<t<\frac{1}{2} \\ 0 & \text { if } t=\frac{1}{2} \\ f(2-2 t) & \text { if } \frac{1}{2}<t<1\end{cases}
$$

for $t \in(0,1)$ and $f \in S$. But $\psi$ is clearly homotopic to zero. Therefore, $[v]=-1_{S}$ and

$$
\left[q \circ i \circ \iota_{u}\right]=1_{S C \mathbf{C}(p)}-S_{1} s_{1}
$$

Therefore,

$$
{ }_{1} \alpha_{2} \cdot{ }_{2} \alpha_{1}=\mathcal{B}_{\mathcal{C} \mathbf{C}(p)} \cdot S^{2}\left(1_{\mathcal{C} \mathbf{C}(p)}-{ }_{1} s_{1}\right) \cdot \mathcal{D}_{\mathcal{C} \mathbf{C}(p)}=1-{ }_{1} s_{1} .
$$


Let $\phi$ denote the action of $\mathbf{C}(p)$ on $C_{u}$. Recall that $e=[1]$ denotes our favorite generator of $\mathbf{C}(p)$.

Definition 11.26. Set

$$
{ }_{2} s_{2}:=\left[\phi_{e}\right] \in \operatorname{KK}\left(C_{u}, C_{u}\right) \text { and }{ }_{2} t_{2}:=\left[\chi_{e}\right] \otimes_{\mathbb{C}} 1_{C_{u}} \in \mathrm{KK}\left(C_{u}, C_{u}\right) .
$$

Lemma 11.27. We have

(1) ${ }_{0} \alpha_{2} \cdot{ }_{2} s_{2}={ }_{0} \alpha_{2},{ }_{2} s_{2} \cdot{ }_{2} \alpha_{0}={ }_{2} \alpha_{0}$;

(2) ${ }_{1} \alpha_{2} \cdot{ }_{2} s_{2}={ }_{1} s_{1} \cdot{ }_{1} \alpha_{2},{ }_{2} s_{2} \cdot{ }_{2} \alpha_{1}={ }_{2} \alpha_{1} \cdot{ }_{1} s_{1}$;

(3) ${ }_{1} \alpha_{2} \cdot{ }_{2} t_{2}={ }_{1} \alpha_{2},{ }_{2} t_{2} \cdot{ }_{2} \alpha_{1}={ }_{2} \alpha_{1}$;

(4) ${ }_{1} \alpha_{2} \cdot{ }_{2} t_{2}={ }_{1} t_{1} \cdot{ }_{1} \alpha_{2},{ }_{2} t_{2} \cdot{ }_{2} \alpha_{1}={ }_{2} \alpha_{1} \cdot{ }_{1} t_{1}$.

Proof. An elementary calculation shows that if $(A, \alpha),(B, \beta)$ are $\mathbf{C}(p)-C^{*}$-algebras and $x \in \operatorname{KK}_{0}^{\mathbf{C}(p)}(A, B)$, then $[\alpha] \cdot x=x \cdot[\beta]$. This shows (1) and (2). Also, the exterior product over $\mathbb{C}$ commutes with every KK-element. Furthermore, in the proof of Lemma 11.16, we have seen that $\left[\chi_{e}\right] \otimes_{\mathbb{C}} 1_{\mathcal{C} \mathbf{C}(p)}=1_{\mathcal{C}(p)}$, this shows (3) and (4).

\subsection{The Subring $\mathbf{K K}_{0}^{\mathbf{C}(p)}\left(C_{u}, C_{u}\right)$}

An immediate consequence of the definition of ${ }_{2} t_{2}$ and ${ }_{2} s_{2}$ is that $\left({ }_{2} s_{2}\right)^{p}=\left({ }_{2} t_{2}\right)^{p}=1_{C_{u}}$ and that ${ }_{2} s_{2}$ and ${ }_{2} t_{2}$ commute. Applying $\operatorname{KK}_{0}^{\mathbf{C}(p)}\left(C_{u}, \ldots\right)$ to the first and the second exact triangle and using the results of Lemma 11.23, we conclude that $K K_{1}^{\mathbf{C}(p)}\left(C_{u}, C_{u}\right)=\{0\}$ and obtain two short exact sequences:

$$
0 \longrightarrow \mathrm{KK}_{1}^{\mathbf{C}(p)}\left(C_{u}, \mathcal{C} \mathbf{C}(p)\right) \stackrel{\left({ }_{1} \alpha_{2}\right)_{*}}{\longrightarrow} \mathrm{KK}_{0}^{\mathbf{C}(p)}\left(C_{u}, C_{u}\right) \stackrel{\left({ }_{2} \alpha_{0}\right)_{*}}{\longrightarrow} \mathrm{KK}_{0}^{\mathbf{C}(p)}\left(C_{u}, \mathbb{C}\right) \longrightarrow 0
$$

and

$$
0 \longrightarrow \mathrm{KK}_{0}^{\mathbf{C}(p)}\left(C_{u}, \mathbb{C}\right) \stackrel{\left({ }_{0} \alpha_{2}\right)_{*}}{\longrightarrow} \mathrm{KK}_{0}^{\mathbf{C}(p)}\left(C_{u}, C_{u}\right) \stackrel{\left({ }_{2} \alpha_{1}\right)_{*}}{\longrightarrow} \mathrm{KK}_{1}^{\mathbf{C}(p)}\left(C_{u}, \mathcal{C} \mathbf{C}(p)\right) \longrightarrow 0 .
$$

Corollary 11.28. $\mathrm{KK}_{*}^{\mathrm{C}(p)}\left(C_{u}, C_{u}\right) \cong \mathbb{Z}^{p-2}[0]$ as an abelian group.

$$
I_{1}:=\operatorname{im}\left({ }_{1} \alpha_{2}\right)_{*}=\operatorname{ker}\left({ }_{2} \alpha_{0}\right)_{*} \text { and } I_{0}:=\operatorname{im}\left({ }_{0} \alpha_{2}\right)_{*}=\operatorname{ker}\left({ }_{1} \alpha_{2}\right)_{*} \text { are left ideals in } \operatorname{KK}_{0}^{\mathbf{C}(p)}\left(C_{u}, C_{u}\right) .
$$

Lemma 11.29. For $i=0,1$,

$$
I_{i} \rightarrow H o m_{\mathbb{Z}}\left(I_{i}, I_{i}\right), \quad a \mapsto(b \mapsto a \cdot b),
$$

is one-to-one. 
Proof. Let us show the statement for $i=1$, for the case $i=0$ just repeat the proof with the indices 1 and 0 interchanged. We have

$$
N\left({ }_{2} t_{2}\right) \cdot{ }_{2} \alpha_{0}={ }_{2} \alpha_{0} \cdot N\left({ }_{0} t_{0}\right)=\left({ }_{2} \alpha_{0} \cdot{ }_{0} \alpha_{1}\right) \cdot{ }_{1} \alpha_{0}=0
$$

by Lemma 11.27. Therefore, $N\left({ }_{2} t_{2}\right) \in I_{1}$. If $a \in I_{1}$, then $a=x \cdot{ }_{1} \alpha_{2}$ for some $x \in$ $\mathrm{KK}_{1}^{\mathbf{C}(p)}\left(C_{u}, \mathcal{C} \mathbf{C}(p)\right)$. Again by Lemma 11.27,

$$
a \cdot N\left({ }_{2} t_{2}\right)=x \cdot \sum_{k=0}^{p-1}{ }_{1} \alpha_{2} \cdot\left({ }_{2} t_{2}\right)^{k}=p \cdot a .
$$

Since $I_{1}$ is torsion-free, the claim follows.

Lemma 11.30. One has

(1) ${ }_{2} \alpha_{1} \cdot{ }_{1} \alpha_{2}=1-{ }_{2} s_{2}$;

(2) ${ }_{2} \alpha_{0} \cdot{ }_{0} \alpha_{2}=1-{ }_{2} t_{2}$.

Proof. We only show the first statement, the second follows by interchanging 1 and 0 . If $y \in I_{1}$, then $y=x^{\prime} \cdot{ }_{1} \alpha_{2}$ for $x^{\prime} \in \mathrm{KK}_{1}^{\mathbf{C}(p)}\left(C_{u}, \mathcal{C} \mathbf{C}(p)\right)$, by Proposition $11.23 x^{\prime}={ }_{2} \alpha_{1} \cdot x^{\prime \prime}$ for some $x^{\prime \prime} \in \mathrm{KK}_{0}^{\mathbf{C}(p)}(\mathcal{C} \mathbf{C}(p), \mathcal{C} \mathbf{C}(p))$, therefore, $y={ }_{2} \alpha_{1} \cdot x$ for some $x \in \mathrm{KK}_{1}^{\mathbf{C}(p)}\left(\mathcal{C} \mathbf{C}(p), C_{u}\right)$. Hence

${ }_{2} \alpha_{1} \cdot{ }_{1} \alpha_{2} \cdot y={ }_{2} \alpha_{1} \cdot\left({ }_{1} \alpha_{2} \cdot{ }_{2} \alpha_{1}\right) \cdot x={ }_{2} \alpha_{1} \cdot\left(1-{ }_{1} s_{1}\right) \cdot x=\left(1-{ }_{2} s_{2}\right) \cdot{ }_{2} \alpha_{1} \cdot x=\left(1-{ }_{2} s_{2}\right) \cdot y$

by Lemma 11.27. By Lemma 11.29, ${ }_{2} \alpha_{1} \cdot{ }_{1} \alpha_{2}=1-{ }_{2} s_{2}$.

Note that Lemma 11.29, Lemma 11.27 and Proposition 11.23 imply that $\mathrm{KK}^{\mathbf{C}(p)}\left(C_{u}, C_{u}\right)$ is generated by ${ }_{2} t_{2}$ and ${ }_{2} s_{2}$ (hence commutative) and that

$$
I_{0}=\left\langle 1-{ }_{2} t_{2}\right\rangle, \quad I_{1}=\left\langle 1-{ }_{2} s_{2}\right\rangle .
$$

Lemma 11.31. One has

(1) $\left(1-{ }_{2} t_{2}\right) \cdot\left(1-{ }_{2} s_{2}\right)=0$;

(2) $N\left({ }_{2} t_{2}\right)+N\left({ }_{2} s_{2}\right)=p$.

Proof. By Lemma 11.27,

$$
{ }_{2} t_{2} \cdot\left(1-{ }_{2} s_{2}\right)=\left({ }_{2} t_{2} \cdot{ }_{2} \alpha_{1}\right) \cdot{ }_{1} \alpha_{2}={ }_{2} \alpha_{1} \cdot{ }_{1} \alpha_{2}=1-{ }_{2} s_{2} .
$$

This shows the first statement.

For the second statement, first calculate

$$
\sum_{k=0}^{p-1}(p-k)\left(1-{ }_{2} s_{2}\right) \cdot\left({ }_{2} s_{2}\right)^{k}=\sum_{k=0}^{p-1}(p-k)\left({ }_{2} s_{2}\right)^{k}-\sum_{k=0}^{p-1}(p-k)\left({ }_{2} s_{2}\right)^{k+1}=p-N\left({ }_{2} s_{2}\right) .
$$


This shows that $p-N\left({ }_{2} s_{2}\right)$ is in $I_{1}$. Since $N\left({ }_{2} s_{2}\right) \cdot\left(1-{ }_{2} s_{2}\right)=0$, we have

$$
\left(p-N\left({ }_{2} s_{2}\right)\right) \cdot\left(1-{ }_{2} s_{2}\right)=p\left(1-{ }_{2} s_{2}\right) .
$$

This implies that $\left(p-N\left({ }_{2} s_{2}\right)\right) y=p y$ for all $y \in I_{1}$. Recall that $N\left({ }_{2} t_{2}\right) \in I_{1}$. Since ${ }_{2} t_{2} \cdot\left(1-{ }_{2} s_{2}\right)=\left(1-{ }_{2} s_{2}\right)$, one has $N\left({ }_{2} t_{2}\right) \cdot y=p y$ for all $y \in I_{1}$. Therefore,

$$
N\left({ }_{2} t_{2}\right) \cdot y=\left(p-N\left({ }_{2} s_{2}\right)\right) \cdot y
$$

for all $y \in I_{1}$. This shows (2) by Lemma 11.29.

Proposition 11.32.

$$
\mathbb{Z}[s, t] /\langle N(t)+N(s)-p,(1-s)(1-t)\rangle \rightarrow \mathrm{KK}_{0}^{\mathbf{C}(p)}\left(C_{u}, C_{u}\right), \quad t \mapsto{ }_{2} t_{2}, s \mapsto{ }_{2} s_{2},
$$

is an isomorphism of rings.

Proof. The previous lemma shows that $t \mapsto{ }_{2} t_{2}, s \mapsto{ }_{2} s_{2}$ gives a well-defined ring homomorphism. Since both rings are free $\mathbb{Z}$-modules of dimension $2 p-2$ it suffices to see that the map is surjective. But we have already observed that $\operatorname{KK}^{\mathbf{C}(p)}\left(C_{u}, C_{u}\right)$ is generated by ${ }_{2} t_{2}$ and ${ }_{2} s_{2}$, this shows the claim.

\subsection{Summary}

Set

$$
A_{0}:=\mathbb{C}, \quad A_{1}:=\mathcal{C} \mathbf{C}(p), \quad A_{2}:=C_{u} .
$$

Let $\mathcal{R}$ be the category ring of the full subcategory of $\mathfrak{K}_{\mathfrak{K}_{*}}^{\mathbf{C}(p)}$ with objects $A_{0}, A_{1}$ and $A_{2}$. In the following ${ }_{i} x_{j}$ will always denote an element in $\operatorname{KK}_{*}{ }^{\mathbf{C}(p)}\left(A_{i}, A_{j}\right)$. Recall that by definition of a category ring we have ${ }_{k} x_{i} \cdot{ }_{j} x_{l}=0$ if $i \neq j$. Let ${ }_{i} 1_{i}=1_{A_{i}}$ denote the unit of $A_{i}$. $\mathcal{R}$ is generated by

$$
{ }_{i} t_{i}, i=0,2, \quad{ }_{i} s_{i}, i=1,2 \text { and }{ }_{i} \alpha_{j}, i, j=0,1,2 .
$$

We have the following relations

1. $1={ }_{0} 1_{0}+{ }_{1} 1_{1}+{ }_{2} 1_{2}$;

2. ${ }_{i} \alpha_{j} \cdot{ }_{j} \alpha_{k}=0$ if $i \neq k$;

3. ${ }_{0} \alpha_{1} \cdot{ }_{1} \alpha_{0}=N\left({ }_{0} t_{0}\right),{ }_{1} \alpha_{0} \cdot{ }_{0} \alpha_{1}=N\left({ }_{1} s_{1}\right)$;

4. ${ }_{0} \alpha_{2} \cdot{ }_{2} \alpha_{0}={ }_{0} 1_{0}-{ }_{0} t_{0},{ }_{1} \alpha_{2} \cdot{ }_{2} \alpha_{1}={ }_{1} 1_{1}-{ }_{1} s_{1}$;

5. ${ }_{2} \alpha_{0} \cdot{ }_{0} \alpha_{2}={ }_{2} 1_{2}-{ }_{2} t_{2},{ }_{2} \alpha_{1} \cdot{ }_{1} \alpha_{2}={ }_{2} 1_{2}-{ }_{2} s_{2}$;

6. $N\left({ }_{2} t_{2}\right)+N\left({ }_{2} s_{2}\right)=p$. 
The grading is given by $\mathcal{R}=\mathcal{R}^{(0)} \oplus \mathcal{R}^{(1)}$, where $\mathcal{R}^{(0)}$ is the subring generated by ${ }_{0} t_{0},{ }_{1} s_{1},{ }_{2} t_{2},{ }_{2} s_{2},{ }_{0} \alpha_{1},{ }_{1} \alpha_{0},{ }_{2} \alpha_{0},{ }_{0} \alpha_{2}$ and $\mathcal{R}^{(1)}$ is the free $\mathbb{Z}$-submodule generated by ${ }_{1} \alpha_{2}$. $\left({ }_{2} t_{2}\right)^{k}$ and $\left({ }_{2} t_{2}\right)^{k} \cdot{ }_{2} \alpha_{1}$ for $k=0, \ldots p-2$.

Moreover, the triangles

$$
\operatorname{SCC}(p) \stackrel{{ }^{1 \alpha_{2}}}{\longrightarrow} C_{u} \stackrel{{ }^{2} \alpha_{0}}{\longrightarrow} \mathbb{C} \stackrel{{ }^{0} \alpha_{1}}{\longrightarrow} \mathcal{C} \mathbf{C}(p)
$$

and

$$
\mathcal{C C}(p) \stackrel{{ }^{\alpha_{0}}}{\longrightarrow} \mathbb{C} \stackrel{{ }^{0 \alpha_{2}}}{\longrightarrow} C_{u} \stackrel{{ }^{2 \alpha_{1}}}{\longrightarrow} S \mathcal{C C}(p)
$$

are exact.

For convenience of the reader and for later reference, we list some more relations, which can easily be derived from the relations above.

1. $\left({ }_{i} t_{i}\right)^{p}={ }_{i} 1_{i},\left({ }_{j} s_{j}\right)^{p}={ }_{j} 1_{j}, \quad i=0,2, \quad j=1,2$;

2. ${ }_{0} t_{0} \cdot{ }_{0} \alpha_{1}={ }_{0} \alpha_{1} \cdot{ }_{1} s_{1}={ }_{0} \alpha_{1}$;

3. ${ }_{1} s_{1} \cdot{ }_{1} \alpha_{0}={ }_{1} \alpha_{0} \cdot{ }_{0} t_{0}={ }_{1} \alpha_{0}$;

4. ${ }_{0} \alpha_{2} \cdot{ }_{2} s_{2}={ }_{0} \alpha_{2},{ }_{2} s_{2} \cdot{ }_{2} \alpha_{0}={ }_{2} \alpha_{0}$;

5. ${ }_{1} \alpha_{2} \cdot{ }_{2} s_{2}={ }_{1} s_{1} \cdot{ }_{1} \alpha_{2},{ }_{2} s_{2} \cdot{ }_{2} \alpha_{1}={ }_{2} \alpha_{1} \cdot{ }_{1} s_{1}$;

6. ${ }_{1} \alpha_{2} \cdot{ }_{2} t_{2}={ }_{1} \alpha_{2},{ }_{2} t_{2} \cdot{ }_{2} \alpha_{1}={ }_{2} \alpha_{1}$;

7. ${ }_{1} \alpha_{2} \cdot{ }_{2} t_{2}={ }_{1} t_{1} \cdot{ }_{1} \alpha_{2},{ }_{2} t_{2} \cdot{ }_{2} \alpha_{1}={ }_{2} \alpha_{1} \cdot{ }_{1} t_{1}$.

\section{Projective Resolutions of $\mathcal{R}$-Modules}

The aim of this section is to show the following theorem:

Theorem 12.1. Let $A$ be a separable $\mathbf{C}(p)-C^{*}$-algebra, then the $\mathcal{R}$-module $\operatorname{EK}^{\mathbf{C}(p)}(A)$ has a projective resolution of length 1 .

Since at first sight, $\mathcal{R}$ seems to be a rather unfamiliar and complicated ring, a first approach is to understand $\mathcal{R}$-modules by looking at certain subrings of $\mathcal{R}$ and decomposing $\mathcal{R}$-modules into modules over these subrings.

To be more precise, let us introduce some notation: Recall that we defined ${ }_{0} 1_{0}$ $\left(1_{1} 1_{1},{ }_{2} 1_{2}\right)$ to be the class of the identity of $\mathbb{C}\left(\mathcal{C} \mathbf{C}(p), C_{u}\right.$ respectively). Define

$$
{ }_{i} \mathcal{R}:={ }_{i} 1_{i} \mathcal{R}, \quad \mathcal{R}_{i}:=\mathcal{R}_{i} 1_{i}, \quad \text { and } \quad{ }_{i} \mathcal{R}_{j}:={ }_{i} 1_{i} \mathcal{R}_{j} 1_{j} .
$$

Since the ${ }_{i} 1_{i}$ 's are idempotents in $\mathcal{R}$ and $1_{\mathcal{R}}={ }_{0} 1_{0}+{ }_{1} 1_{1}+{ }_{2} 1_{2}$, every $\mathcal{R}$-module $M$ has a decomposition as abelian groups

$$
M={ }_{0} M \oplus{ }_{1} M \oplus_{2} M \text { with }{ }_{i} M:={ }_{i} 1_{i} M={ }_{i} \mathcal{R} \otimes_{\mathcal{R}} M .
$$


Note that every $x \in{ }_{i} \mathcal{R}_{j}$ yields a morphism ${ }_{j} M \stackrel{x^{*}}{\rightarrow}{ }_{i} M$, in $\operatorname{particular}_{i} M$ is a left module over ${ }_{i} \mathcal{R}_{i}$.

It is not true that all $\mathcal{R}$-modules have projective dimension 1. Fortunately, we are only interested in modules of the form $\operatorname{EK}^{\mathbf{C}(p)}(A)$ and these modules have an extra property. The decomposition for these modules looks as follows

$$
\begin{gathered}
{ }_{0} \mathrm{EK}^{\mathbf{C}(p)}(A)=\mathrm{KK}_{*}{ }^{\mathbf{C}(p)}(\mathbb{C}, A),{ }_{1} \mathrm{EK}^{\mathbf{C}(p)}(A)=\mathrm{KK}_{*}{ }^{\mathbf{C}(p)}(\mathcal{C} \mathbf{C}(p), A), \\
{ }_{2} \mathrm{EK}^{\mathbf{C}(p)}(A)=\mathrm{KK}_{*}{ }^{\mathbf{C}(p)}\left(C_{u}, A\right) .
\end{gathered}
$$

Since the triangles

$$
\operatorname{SCC}(p) \stackrel{{ }^{1 \alpha_{2}}}{\longrightarrow} C_{u} \stackrel{{ }^{2} \alpha_{0}}{\longrightarrow} \mathbb{C} \stackrel{{ }^{\alpha_{1}}}{\longrightarrow} \mathcal{C} \mathbf{C}(p), \quad \mathcal{C} \mathbf{C}(p) \stackrel{{ }^{1 \alpha_{0}}}{\longrightarrow} \mathbb{C} \stackrel{{ }^{0 \alpha_{2}}}{\longrightarrow} C_{u} \stackrel{{ }^{2 \alpha_{1}}}{\longrightarrow} S \mathcal{C} \mathbf{C}(p)
$$

are exact, $\operatorname{EK}^{\mathbf{C}(p)}(A)$ is exact in the following sense:

Definition 12.2. An $\mathcal{R}$-module $M$ is called exact if the sequences
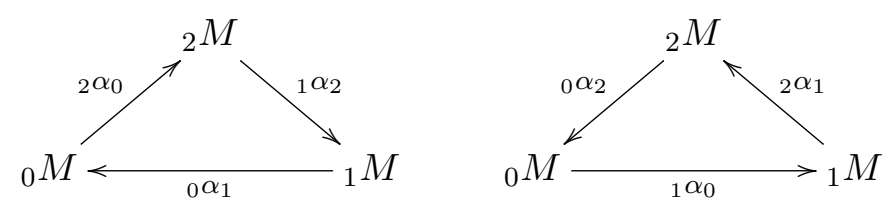

are exact.

In particular, $\mathcal{R}$ is exact.

Definition 12.3. If $R$ is a ring and $M$ is a module over $R$, which is free as an abelian group, let us call $M$ a lattice.

Note that we do not assume $M$ to be finitely generated.

We will prove that every exact $\mathcal{R}$-lattice is projective. This implies that every exact $\mathcal{R}$-module has a projective resolution of length 1 by the following argument: If $M \longmapsto$ $M^{\prime} \rightarrow M^{\prime \prime}$ is a short exact sequence of $\mathcal{R}$-modules, of which two modules are exact, then the long exact sequence for homology of a short exact sequence of chain complexes shows that the third module is exact as well. If $M$ is an $\mathcal{R}$-module, there is a projection $\bigoplus_{I} \mathcal{R} \rightarrow M$ from a free $\mathcal{R}$-module onto $M$. Its kernel $K$ is an $\mathcal{R}$-lattice, furthermore $K$ is exact if and only if $M$ is exact. If $K$ is projective, then of course $M$ has a projective resolution of length 1 . In fact we will prove Theorem 12.1 by showing the following statement:

Theorem 12.4. Let $L$ be a countably generated, exact $\mathcal{R}$-lattice, then $L$ is projective.

Note that it is easy to see that projective $\mathcal{R}$-modules are exact lattices since direct summands of exact lattices are exact lattices and $\mathcal{R}$ is an exact lattice itself.

As indicated above, we will analyze exact $\mathcal{R}$-lattices by first investigating lattices over the subrings ${ }_{0} \mathcal{R}_{0},{ }_{1} \mathcal{R}_{1}$ and ${ }_{2} \mathcal{R}_{2}$. We have

$$
{ }_{0} \mathcal{R}_{0} \cong{ }_{1} \mathcal{R}_{1} \cong \mathbb{Z} \mathbf{C}(p) \cong \mathbb{Z}[t] /\left\langle t^{p}-1\right\rangle
$$


and

$$
{ }_{2} \mathcal{R}_{2} \cong \mathbb{Z}[t, s] /\langle N(t)+N(s)-p,(1-t)(1-s)\rangle .
$$

There is a classical structure result on finitely generated $\mathbb{Z} \mathbf{C}(p)$-lattices by I. Reiner [38], which was extended to the countably generated case by M. C. R. Butler, J. M. Campbell and L. G. Kovács [8]. It uses that multiplication by $N(t)$ and $t-1$ induces ring extensions

$$
\mathbb{Z}\left[\theta_{p}\right] \longmapsto \mathbb{Z} \mathbf{C}(p) \rightarrow \mathbb{Z} \text { and } \mathbb{Z} \longmapsto \mathbb{Z} \mathbf{C}(p) \rightarrow \mathbb{Z}\left[\theta_{p}\right],
$$

where $\mathbb{Z}\left[\theta_{p}\right]$ denotes $\mathbb{Z}$ with a $p$ th root of unity adjoint. $\mathbb{Z}\left[\theta_{p}\right]$ is a Dedekind ring, and lattices over these rings have a good structure theory. This can be used to analyze lattices over $\mathbb{Z} \mathbf{C}(p)$. We will first recall some basic theory of Dedekind rings and lattices over them and then move on to lattices over $\mathbb{Z} \mathbf{C}(p)$. The main result here is that every $\mathbb{Z} \mathbf{C}(p)$-lattice can be decomposed into a direct sum of a projective lattice and lattices which are pullbacks of the projections $\mathbb{Z} \mathbf{C}(p) \rightarrow \mathbb{Z}$ and $\mathbb{Z} \mathbf{C}(p) \rightarrow \mathbb{Z}\left[\theta_{p}\right]$. Furthermore, we characterize projective $\mathbb{Z} \mathbf{C}(p)$-modules as lattices satisfying a certain exactness property. Fortunately, many ideas from the classification results can be carried over to lattices over ${ }_{2} \mathcal{R}_{2}$. We obtain a similar characterization of projective ${ }_{2} \mathcal{R}_{2}$-modules as in the case of modules over $\mathbb{Z} \mathbf{C}(p)$. Finally, we show that $\mathcal{R}$-lattices, which are induced from projective lattices over one of the subrings ${ }_{i} \mathcal{R}_{i}$, are again projective and that every exact $\mathcal{R}$-lattice can be decomposed as a direct sum of projective lattices of this kind. This will then prove Theorem 12.1.

\subsection{Conventions and Generalities on Rings and Modules}

Throughout this subsection, let $R$ be a ring. Let $r \in R$ and $M$ be an $R$-module. Set

$$
M_{r}:=\{m \in M \mid r m=0\} .
$$

Definition 12.5. Let $M$ be an $R$-module, $M$ is called torsion-free if $M_{r} \neq\{0\}$ implies $r=0$. Let $N$ a submodule of $M$. $N$ is called a pure submodule if for every $r \in R$

$$
r N=N \cap r M .
$$

We will need an elementary linear algebra fact which we state as a separate lemma. Let $A \in \mathbb{M}_{n}(R)$ and $S \in \mathbb{M}_{n}(\mathbb{Z})$, we define the product $S A \in \mathbb{M}_{n}(R)$ by regarding $R$ as a left $\mathbb{Z}$-module.

Lemma 12.6. Let $R$ be a ring, $\phi: R \rightarrow \mathbb{Z} / p$ an epimorphism of rings and $\phi_{n}: \mathbb{M}_{n}(R) \rightarrow$ $\mathbb{M}_{n}(\mathbb{Z} / p)$ its amplification. If $A \in \mathbb{M}_{n}(R)$ is such that $\phi_{n}(A) \in G L_{n}(\mathbb{Z} / p)$ then there is $S \in G L_{n}(\mathbb{Z})$ such that $\phi_{n}(S A) \in G L_{n}(\mathbb{Z} / p)$ is diagonal with non-zero entries on the diagonal.

Proof. We obviously have $\phi_{n}(S A)=S \phi_{n}(A)$, therefore, it suffices to show the statement for $\phi=\operatorname{id}_{\mathbb{Z} / p}$. Let $A \in G L_{n}(\mathbb{Z} / p)$. First note that the elementary row operations of changing rows and adding an integral multiple of one row to another can be realized by multiplication by matrices $S \in G L_{n}(\mathbb{Z})$ from the left. Using the gaussian elimination 
algorithm, we find $S^{\prime} \in G L_{n}(\mathbb{Z})$ such that $S^{\prime} A$ is upper triangular with non-zero entries on the diagonal. (Note that we do not have to multiply rows by integers during the gaussian elimination algorithm since we are working over $\mathbb{Z} / p$.) Performing further elementary row operations, we find $S^{\prime \prime} \in G L_{n}(\mathbb{Z})$ such that $S^{\prime \prime} S^{\prime} A$ is diagonal with non-zero entries on the diagonal.

\subsection{Preliminaries on Dedekind Domains}

Recall that a Dedekind domain is an integral domain $D$ which satisfies

1. $D$ is noetherian,

2. every prime ideal in $D$ is maximal,

3. $D$ is integrally closed in in its quotient field $K$.

Throughout this subsection, let $D$ denote a Dedekind domain with quotient field $K$. Let us state two classical facts about ideals in Dedekind domains.

Theorem 12.7. Every proper non-zero ideal in D factors as a product of prime ideals, which are unique up to reordering.

Proof. [11] 18.6.

Lemma 12.8. Let $I_{1}$ and $I_{2}$ be ideals in $D$, then $I_{1} \subseteq I_{2}$ if and only if there is an ideal $J$ such that $I_{1}=J I_{2}$.

Proof. [11] 18.11.

Let us collect some facts about modules over a Dedekind domain.

Definition 12.9. A fractional ideal $\mathfrak{A}$ is a finitely generated $D$-submodule of $K$. The $D$-rank of a $D$-module $M$ is given by $\operatorname{dim}_{K} K \otimes_{D} M$.

Note that every ideal is a fractional ideal since $D$ is noetherian.

Theorem 12.10. Let $L$ be a torsion-free $D$-module of $D$-rank $n$, then $L$ is the direct sum of $n$ fractional ideals.

Proof. [11] 22.5 .

Lemma 12.11. Let $P$ be a finitely generated torsion-free $D$-module, then $P$ is projective.

Proof. There is a $D$-module epimorphism $\rho: D^{n} \rightarrow P$. Since $P$ is torsion-free, ker $\rho$ is a pure submodule of $D^{n}$. Hence $\operatorname{ker} \rho$ is a direct summand of $D^{n}$ by [11] 22.15. This shows that $P$ is a direct summand of $D^{n}$.

In particular, fractional ideals are projective.

Let $\theta_{p}$ be a primitive $p$ th root of unity. The cyclotomic field $\mathbb{Q}\left[\theta_{p}\right]$ is the finite field extension of $\mathbb{Q}$ by $\theta_{p}$. Let $\mathbb{Z}\left[\theta_{p}\right]$ be the subring generated by $\mathbb{Z}$ and $\theta_{p}$. As noted above, $\mathbb{Z}\left[\theta_{p}\right]$ is a quotient of $\mathbb{Z} \mathbf{C}(p)$. 
Theorem 12.12. $\mathbb{Z}\left[\theta_{p}\right]$ is a Dedekind domain with quotient field $\mathbb{Q}\left[\theta_{p}\right]$.

Proof. By $[11]$ Theorem $21.13, \mathbb{Z}\left[\theta_{p}\right]$ is the ring of algebraic integers of $\mathbb{Q}\left[\theta_{p}\right]$, hence $\mathbb{Q}\left[\theta_{p}\right]$ is the quotient field of $\mathbb{Z}\left[\theta_{p}\right]$ ([11] 17.8) and $\mathbb{Z}\left[\theta_{p}\right]$ is a Dedekind domain ([11] 17.12, 12.13 and 18.3).

The following statements will be used at some points.

Lemma 12.13. Let $\mathfrak{A}$ be a non-zero fractional ideal of $\mathbb{Z}\left[\theta_{p}\right]$, then

(1) $\mathfrak{A} /\left(\theta_{p}-1\right) \mathfrak{A} \neq\{0\}$,

(2) $\mathfrak{A}$ has $\mathbb{Z}$-rank $p-1$,

(3) there is $n \in \mathbb{N}, n \geq 2$ and fractional ideals $\mathfrak{A}_{2}, \ldots, \mathfrak{A}_{n}$ such that

$$
\mathfrak{A} \oplus \bigoplus_{i=2}^{n} \mathfrak{A}_{i} \cong \mathbb{Z}\left[\theta_{p}\right]^{n}
$$

Proof. It is easy to see that for every fractional ideal $\mathfrak{A} \subseteq \mathbb{Q}\left[\theta_{p}\right]$, there is $r \in \mathbb{Z}\left[\theta_{p}\right]$ such that $r \cdot \mathfrak{A} \subseteq \mathbb{Z}\left[\theta_{p}\right]$. Now $\left(\theta_{p}-1\right) r \mathfrak{A} \neq r \mathfrak{A}$ by Theorem 12.7. This shows $\mathfrak{A} \neq\left(\theta_{p}-1\right) \mathfrak{A}$ and thereby (1). Note that (.) $\otimes_{\mathbb{Z}\left[\theta_{p}\right]} \mathbb{Q}\left[\theta_{p}\right]=(.) \otimes_{\mathbb{Z}} \mathbb{Q}$. Hence the $\mathbb{Z}$-rank of $\mathfrak{A}$ is equal to $\operatorname{dim}_{\mathbb{Q}}\left(\mathfrak{A} \otimes_{\mathbb{Z}} \mathbb{Q}\right)=\operatorname{dim}_{\mathbb{Q}}\left(\mathbb{Q}\left[\theta_{p}\right]\right)=p-1$. This shows $(2)$.

(3) follows from Lemma 12.11 and Theorem 12.10.

Finally, we will prove a useful characterization of projective $\mathbb{Z}\left[\theta_{p}\right]$-modules.

Theorem 12.14. Let $M$ be a countably generated $\mathbb{Z}\left[\theta_{p}\right]$-module, then the following are equivalent:

(1) $M$ is projective.

(2) $M$ is a lattice.

(3) $M$ is a direct sum of fractional ideals.

Proof. (1) $\Rightarrow(2)$ : A direct summand of $\bigoplus_{I} \mathbb{Z}\left[\theta_{p}\right]$ is of course a lattice.

$(2) \Rightarrow(3)$ : Let us first show that a lattice $L$ is torsion-free: For $x \in L$, the annihilator of $x$

$$
\operatorname{Ann}(x)=\left\{r \in \mathbb{Z}\left[\theta_{p}\right] \mid r x=0\right\}
$$

is an ideal in $\mathbb{Z}\left[\theta_{p}\right]$ and $r \mapsto r x$ induces a monomorphism

$$
\mathbb{Z}\left[\theta_{p}\right] / \operatorname{Ann}(x) \longmapsto L
$$

Let us assume that $\operatorname{Ann}(x) \neq\{0\}$. Then $\operatorname{Ann}(x)$ is a non-zero fractional ideal. Since the $\mathbb{Z}$-rank of $\operatorname{Ann}(x)$ is $p-1, \mathbb{Z}\left[\theta_{p}\right] / \operatorname{Ann}(x)$ is finite. Hence the fact that $L$ is a lattice implies that $\operatorname{Ann}(x)=\mathbb{Z}\left[\theta_{p}\right]$, which shows that $x=0$. 
Claim 12.15. Let $L$ be a $\mathbb{Z}\left[\theta_{p}\right]$-lattice and $x \in L$. Then there is a projective submodule $L^{\prime}$ such that $x \in L^{\prime}$ and $L / L^{\prime}$ is a lattice.

Define $L^{\prime}$ to be the pure $\mathbb{Z}$-closure of $\mathbb{Z}\left[\theta_{p}\right] x$, i.e.,

$$
L^{\prime}=\left\{y \in L \mid \exists n \in \mathbb{Z}: n y \in \mathbb{Z}\left[\theta_{p}\right] x\right\} .
$$

Then of course $x \in L^{\prime}$. Since $L^{\prime}$ is a lattice it is torsion-free. $L$ is free as an abelian groups, therefore, there is a countable index set $I$ and an isomorphism of abelian groups $\phi: L \cong \bigoplus_{I} \mathbb{Z}$. Since $\mathbb{Z}\left[\theta_{p}\right] x$ is finitely generated, there is a finite index set $I_{0} \subseteq I$ such that $\Phi\left(\mathbb{Z}\left[\theta_{p}\right] x\right) \subset \bigoplus_{I_{0}} \mathbb{Z}$, hence also $\Phi\left(L^{\prime}\right) \subseteq \bigoplus_{I_{0}} \mathbb{Z}$. This shows that $L^{\prime}$ is finitely generated and therefore, a projective $\mathbb{Z}\left[\theta_{p}\right]$-module by Lemma 12.11. By assumption there is an isomorphism of abelian groups $\phi: L \cong \bigoplus_{I} \mathbb{Z}$. Since $L^{\prime}$ is pure as $\mathbb{Z}$-module in $L$, a base change in $\bigoplus_{I_{0}} \mathbb{Z}$ shows that $L / L^{\prime}$ is a lattice as well. This shows the claim.

Now let $\left(x_{n}\right)_{n \in \mathbb{N}}$ be a sequence such that $\left\{x_{n} \mid n \in \mathbb{N}\right\}$ generates $L$. By the previous claim, there is an increasing sequence of finitely generated submodules $\left(L_{n}\right)_{n \in \mathbb{N}}$ with $x_{n} \in L_{n}$ such that $L / L_{n}$ is a lattice. $L_{1}$ and $L_{n+1} / L_{n}$ for $n \in \mathbb{N}$ are finitely generated lattices, therefore, direct sums of fractional ideals. Using the exact sequences

$$
L_{n} \longmapsto L_{n+1} \rightarrow L_{n+1} / L_{n}
$$

and the fact that fractional ideals are projective, we see that there is a sequence of fractional ideals $\mathfrak{A}_{i}, i \in \mathbb{N}$ and a monotone map $f: \mathbb{N} \rightarrow \mathbb{N} \cup\{0\}$ such that

$$
L_{n} \cong \bigoplus_{i=1}^{f(n)} \mathfrak{A}_{i} .
$$

Since $L=\bigcup_{n \in \mathbb{N}} L_{n}$, this shows $(2) \Rightarrow(3)$.

$(3) \Rightarrow(1)$ follows from the fact that fractional ideals are projective.

\subsection{Lattices over $\mathbb{Z} \mathbf{C}(p)$}

A $\mathbb{Z}\left[\theta_{p}\right]$-module $M$ can always be viewed as a $\mathbb{Z} \mathbf{C}(p)$-module by setting $t \cdot m:=\theta_{p} m$. This is just the pullback via the quotient map $\mathbb{Z} \mathbf{C}(p) \rightarrow \mathbb{Z}\left[\theta_{p}\right]$. Another way of constructing $\mathbb{Z} \mathbf{C}(p)$-modules from $\mathbb{Z}\left[\theta_{p}\right]$-modules is the following: Let $M$ be a $\mathbb{Z}\left[\theta_{p}\right]$-module and $\underline{m}:=$ $\left(m_{1}, \ldots m_{n}\right) \in M^{n}$. Define a $\mathbb{Z} \mathbf{C}(p)$-module $(M, \underline{m})$ via

$$
(M, \underline{m}):=M \oplus \mathbb{Z}^{n} \text { as abelian groups }, t \cdot(m, k)=\left(\theta_{p} m+k \cdot \underline{m}^{T}, k\right),
$$

here $k \cdot \underline{m}^{T}$ denotes matrix multiplication of the row vector $k$ with the column vector $\underline{m}^{T} .(M, \underline{m})$ is well-defined since

$$
t^{p} \cdot(m, k)=\left(\left(\theta_{p}\right)^{p} m^{T}+N\left(\theta_{p}\right) k \cdot \underline{m}^{T}, k\right)=(m, k) .
$$

Note that $N\left(\theta_{p}\right)=0$ in $\mathbb{Z}\left[\theta_{p}\right]$. It is clear that $(M, \underline{m})$ is a lattice (finitely generated lattice) if and only if $M$ is a lattice (finitely generated lattice, respectively).

I. Reiner [38] showed that every indecomposable, finitely generated $\mathbb{Z} \mathbf{C}(p)$-lattice is of one of the following types: 
1. $\mathbb{Z}$ with trivial $\mathbb{Z} \mathbf{C}(p)$-action;

2. $\mathfrak{A}$ for a fractional $\mathbb{Z}\left[\theta_{p}\right]$-ideal $\mathfrak{A}$ with a $\mathbb{Z} \mathbf{C}(p)$-action given by a pullback as above;

3. ( $\left.\mathfrak{A}, a_{0}\right)$ for a fractional $\mathbb{Z}\left[\theta_{p}\right]$-ideal and $a_{0} \in \mathfrak{A} \backslash\left(\theta_{p}-1\right) \mathfrak{A}$.

Furthermore, I. Reiner showed that every finitely generated $\mathbb{Z} \mathbf{C}(p)$-lattice decomposes into a direct sum of these indecomposables.

In [8], M. C. R. Butler, J. M. Campbell and L. G. Kovács extend this result to infinitely generated $\mathbb{Z} \mathbf{C}(p)$-lattices:

Theorem 12.16. Let $L$ be a countably generated $\mathbb{Z} \mathbf{C}(p)$-lattice, then there are countable index sets $I_{1}, I_{2}, I_{3}$, fractional $\mathbb{Z}\left[\theta_{p}\right]$-ideals $\mathfrak{A}_{i}$ for $i \in I_{2} \sqcup I_{3}$ and $a_{i} \in \mathfrak{A}_{i} \backslash\left(\theta_{p}-1\right) \mathfrak{A}_{i}$ for $i \in I_{3}$ such that

$$
L \cong \bigoplus_{I_{1}} \mathbb{Z} \oplus \bigoplus_{I_{2}} \mathfrak{A}_{i} \oplus \bigoplus_{I_{3}}\left(\mathfrak{A}_{i}, a_{i}\right) .
$$

Next we will characterize projective $\mathbb{Z} \mathbf{C}(p)$-modules. But first we need a preliminary lemma.

Lemma 12.17. Let $x_{1}, \ldots x_{n} \in \mathbb{Z}\left[\theta_{p}\right]^{n}$ and set $\underline{x}=\left(x_{1}, \ldots x_{n}\right)$. Assume that $\left[x_{1}\right], \ldots\left[x_{n}\right]$ is a $\mathbb{Z} / p$-basis of

$$
\mathbb{Z}\left[\theta_{p}\right]^{n} /\left(\theta_{p}-1\right) \mathbb{Z}\left[\theta_{p}\right]^{n} \cong(\mathbb{Z} / p)^{n}
$$

Then

$$
\left(\mathbb{Z}\left[\theta_{p}\right]^{n}, \underline{x}\right) \cong(\mathbb{Z} \mathbf{C}(p))^{n}
$$

as $\mathbb{Z} \mathbf{C}(p)$-modules.

Proof. View elements $x \in \mathbb{Z}\left[\theta_{p}\right]^{n}$ as column vectors and $\underline{x}=\left(x_{1}, \ldots x_{n}\right)$ as an element in $\mathbb{M}_{n}\left(\mathbb{Z}\left[\theta_{p}\right]\right)$. For $M \in \mathbb{M}_{n}\left(\mathbb{Z}\left[\theta_{p}\right]\right)$, let $[M]$ denote the corresponding element in the quotient ring

$$
\mathbb{M}_{n}\left(\mathbb{Z}\left[\theta_{p}\right] /\left(\theta_{p}-1\right) \mathbb{Z}\left[\theta_{p}\right] \cong \mathbb{M}_{n}(\mathbb{Z} / p) .\right.
$$

By Lemma 12.6, there is $S \in G L_{n}(\mathbb{Z})$ such that $[S \cdot \underline{x}] \in \mathbb{M}_{n}(\mathbb{Z} / p)$ is diagonal with non-zero entries on the diagonal. Then

$$
S: \mathbb{Z}\left[\theta_{p}\right]^{n} \rightarrow \mathbb{Z}\left[\theta_{p}\right]^{n}, \quad x \mapsto S \cdot x
$$

is an isomorphism of $\mathbb{Z}\left[\theta_{p}\right]$-modules, and there are $c_{i} \in \mathbb{Z}, c_{i} \neq 0 \bmod p$ and $z_{i} \in \mathbb{Z}\left[\theta_{p}\right]^{n}$ such that

$$
\underline{x}^{\prime}:=\left(S\left(x_{1}\right), \ldots, S\left(x_{n}\right)\right)=\left(c_{1} e_{1}+\left(\theta_{p}-1\right) z_{1}, \ldots, c_{n} e_{n}+\left(\theta_{p}-1\right) z_{n}\right) .
$$

Her, $e_{i} \in \mathbb{Z}\left[\theta_{p}\right]^{n}$ denotes the column vector with 1 in the $i$ th entry and zeros elsewhere. Then

$$
(S, 1):\left(\mathbb{Z}\left[\theta_{p}\right]^{n}, \underline{x}\right) \rightarrow\left(\mathbb{Z}\left[\theta_{p}\right]^{n}, \underline{x}^{\prime}\right), \quad(S, 1)(x, y):=(S(x), y)
$$

is an isomorphism. This shows that it suffices to consider the case $x_{i}=c_{i} e_{i}+\left(\theta_{p}-1_{i}\right) z_{i}$ with $c_{i}, e_{i}$ and $z_{i}$ as above. 
Set $\underline{z}:=\left(z_{1}, \ldots, z_{n}\right)$ and $\underline{c}=\left(c_{1} e_{1}, \ldots c_{n} e_{n}\right)$ and define

$$
\Psi:\left(\mathbb{Z}\left[\theta_{p}\right]^{n}, \underline{c}\right) \rightarrow\left(\mathbb{Z}\left[\theta_{p}\right]^{n}, \underline{x}\right), \quad \Psi(a, m)=\left(a-m^{T} \cdot \underline{z}, m\right) .
$$

The calculation

$$
\begin{aligned}
\Psi(t \cdot(a, m)) & =\Psi\left(\theta_{p} a+m^{T} \cdot \underline{c}, m\right)=\left(\theta_{p} a+m^{T} \cdot(\underline{c}-\underline{z}), m\right) \\
& =\left(\theta_{p} a+m^{T} \cdot\left(\underline{x}-\theta_{p} \underline{z}\right), m\right)=\left(\theta_{p}\left(a-m^{T} \cdot \underline{z}\right)+m^{T} \cdot \underline{x}, m\right) \\
& =t \cdot \Psi(a, m)
\end{aligned}
$$

shows that $\Psi$ is an isomorphism of $\mathbb{Z} \mathbf{C}(p)$-modules. For $c \in \mathbb{Z}, c \neq 0 \bmod p$, there is an isomorphism $\left(\mathbb{Z}\left[\theta_{p}\right], c\right) \cong\left(\mathbb{Z}\left[\theta_{p}\right], 1\right)\left([11]\right.$, Lemma 74.2.) and $\left(\mathbb{Z}\left[\theta_{p}\right], 1\right)$ is isomorphic to $\mathbb{Z} \mathbf{C}(p)$ via

$$
\mathbb{Z} \mathbf{C}(p) \rightarrow\left(\mathbb{Z}\left[\theta_{p}\right], 1\right), \quad t^{k} \mapsto\left(\sum_{i=0}^{k-1} \theta^{i}, 1\right) .
$$

This shows

$$
\left(\mathbb{Z}\left[\theta_{p}\right]^{n}, \underline{x}\right) \cong\left(\mathbb{Z}\left[\theta_{p}\right]^{n}, \underline{c}\right) \cong \bigoplus_{i=1}^{n}\left(\mathbb{Z}\left[\theta_{p}\right], c_{i}\right) \cong \mathbb{Z} \mathbf{C}(p)^{n}
$$

and proves the claim.

Definition 12.18. Let $M$ be a $\mathbb{Z} \mathbf{C}(p)$-module. $M$ is called exact if $M_{N(t)}=(t-1) M$ and $M_{t-1}=N(t) M$.

Theorem 12.19. Let $M$ be a countably generated $\mathbb{Z} \mathbf{C}(p)$-module. Then the following are equivalent:

(1) There is a countable index set $I$ and for all $i \in I$, there are fractional $\mathbb{Z}\left[\theta_{p}\right]$-s ideals $\mathfrak{A}_{i}$ and $a_{i} \in \mathfrak{A}_{i} \backslash\left(\theta_{p}-1\right) \mathfrak{A}_{i}$ such that

$$
L \cong \bigoplus_{I}\left(\mathfrak{A}_{i}, a_{i}\right)
$$

(2) $M$ is projective.

(3) $M$ is an exact $\mathbb{Z} \mathbf{C}(p)$-lattice.

Proof. $(1) \Rightarrow(2)$ : Let $\mathfrak{A}_{1}$ be a fractional ideal and $a_{1} \in \mathfrak{A}_{1} \backslash\left(\theta_{p}-1\right) \mathfrak{A}_{1}$, we have to show that $\left(\mathfrak{A}_{1}, a_{1}\right)$ is a projective $\mathbb{Z} \mathbf{C}(p)$-module.

By Lemma 12.13 , there are $n \in \mathbb{N}, n \geq 2$, fractional ideals $\mathfrak{A}_{2}, \ldots, \mathfrak{A}_{n}$ and an isomorphism

$$
T: \bigoplus_{i=1}^{n} \mathfrak{A}_{i} \cong \mathbb{Z}\left[\theta_{p}\right]^{n}
$$

of $\mathbb{Z}\left[\theta_{p}\right]$-modules. By Lemma 12.13 , we may choose $a_{i} \in \mathfrak{A}_{i}$ such that $\left[a_{i}\right] \neq 0 \in$ $\mathfrak{A}_{i} /\left(\theta_{p}-1\right) \mathfrak{A}_{i} .\left\{\left[a_{1}\right], \ldots,\left[a_{n}\right]\right\}$ is a basis of the $\mathbb{Z} / p$-vector space

$$
\bigoplus_{i=1}^{n} \mathfrak{A}_{i} \mid\left(\theta_{p}-1\right) \bigoplus_{i=1}^{n} \mathfrak{A}_{i} \cong(\mathbb{Z} / p) .
$$


Set $x_{i}:=T\left(a_{i}\right), \underline{a}:=\left(a_{1}, \ldots, a_{n}\right)$ and $\underline{x}=\left(x_{1}, \ldots, x_{n}\right)$, then

$$
(T, 1):\left(\bigoplus_{i=1}^{n} \mathfrak{A}_{i}, \underline{y}\right) \rightarrow\left(\mathbb{Z}\left[\theta_{p}\right]^{n}, \underline{x}\right), \quad(a, b) \mapsto(T(a), b) .
$$

is an isomorphism of $\mathbb{Z} \mathbf{C}(p)$-modules and $\left(\mathbb{Z}\left[\theta_{p}\right]^{n}, \underline{x}\right)$ is isomorphic to $(\mathbb{Z} \mathbf{C}(p))^{n}$ by Lemma 12.17. This finishes the proof of $(1) \Rightarrow(2)$.

$(2) \Rightarrow(3)$ : Note that $\mathbb{Z} \mathbf{C}(p)$ is an exact lattice. This implies the claim since direct summands of exact $\mathbb{Z} \mathbf{C}(p)$-lattices are again exact $\mathbb{Z} \mathbf{C}(p)$-lattices.

$(3) \Rightarrow(1)$ : Let

$$
L \cong \bigoplus_{I_{1}} \mathbb{Z} \oplus \bigoplus_{I_{2}} \mathfrak{A}_{i} \oplus \bigoplus_{I_{3}}\left(\mathfrak{A}_{i}, a_{i}\right)
$$

be a decomposition as in Theorem 12.16. It is easy to check that indecomposable modules of the type $\mathbb{Z}$ or $\mathfrak{A}$ are not exact. This finishes the proof.

We can draw the following conclusion from Theorem 12.16:

Theorem 12.20. Let $L$ be a $\mathbb{Z} \mathbf{C}(p)$-lattice, then there are $\mathbb{Z} \mathbf{C}(p)$-sublattices $L_{1}, L_{2}$ and $L_{3}$ such that $L=L_{1} \oplus L_{2} \oplus L_{3}$ and

(1) $L_{1} \subseteq L_{t-1} \subseteq L_{1} \oplus L_{3}$;

(2) $L_{2} \subseteq L_{N(t)} \subseteq L_{2} \oplus L_{3}$;

(3) $L_{3}$ is projective and exact.

Proof. Let

$$
\phi: L \cong \bigoplus_{I_{1}} \mathbb{Z} \oplus \bigoplus_{I_{2}} \mathfrak{A}_{i} \oplus \bigoplus_{I_{3}}\left(\mathfrak{A}_{i}, a_{i}\right)
$$

be a decomposition as in Theorem 12.16. Set $L_{1}:=\phi^{-1}\left(\bigoplus_{I_{1}} \mathbb{Z}\right) L_{2}:=\phi^{-1}\left(\bigoplus_{I_{2}} \mathfrak{A}_{i}\right)$ and $L_{3}:=\phi^{-1}\left(\bigoplus_{I_{3}}\left(\mathfrak{A}_{i}, a_{i}\right)\right)$. Obviously $L_{1} \subseteq L_{t-1}$ and $L_{2} \subseteq L_{N(t)}$. We have $L_{t-1}=$ $\bigoplus_{i=1}^{3} L_{t-1} \cap L_{i}$ and $L_{N(t)}=\bigoplus_{i=1}^{3} L_{N(t)} \cap L_{i}$. Since $L_{N(t)} \cap L_{t-1}=\{0\}$, we get $L_{t-1} \subseteq$ $L_{1} \oplus L_{3}$ and $L_{N(t)} \subseteq L_{2} \oplus L_{3}$. (3) follows by the previous results of this section.

\subsection{Lattices over ${ }_{2} \mathcal{R}_{2}$}

Recall that there is an isomorphism

$$
{ }_{2} \mathcal{R}_{2} \cong \mathbb{Z}[t, s] /\langle N(t)+N(s)-p,(1-t)(1-s)\rangle .
$$

For ease of notation, let us denote the ring on the right-hand-side by $R$. There is an involution $\gamma$ on $R$, which is given by $s \mapsto t$. In other words, $R$ is symmetric in $t$ and $s$.

Lemma 12.21. Let $M$ be an $R$-module, then $M_{s-1} \cap M_{t-1} \subseteq M_{p}$. In particular, if $M$ is torsion-free, then $M_{s-1} \cap M_{t-1}=\{0\}$.

Proof. If $m \in M$ with $m=t m=s m$, then $p m=N(t) m+N(s) m=2 p m$, hence $p m=0$. 
Lemma 12.22. We have

$$
(t-1) R=R_{s-1} \text { and }(s-1) R=R_{t-1}
$$

and $(t-1) R$ and $(s-1) R$ are isomorphic to $\mathbb{Z}\left[\theta_{p}\right]$.

Proof. A $\mathbb{Z}$-basis of $R$ is given by $\left\{1, s, \ldots, s^{p-1},(t-1), t(t-1), \ldots, t^{p-3}(t-1)\right\}$ (by $\{1, s\}$ if $p=2)$. Hence, if $r \in R$ with $(s-1) r=0$, we can write $r$ as a $\mathbb{Z}$-linear combination with respect to this basis to see that there is $n \in \mathbb{N}$ and $r^{\prime} \in R$ such that $r=n N(s)+(t-1) r^{\prime}$. But

$$
n N(s)=n(N(t)-p)=n(t-1) \sum_{i=0}^{p-1}(p-i) t^{i} .
$$

This shows $(t-1) R=R_{s-1}$. Since $R$ is symmetric in $t$ and $s$, we see that $(s-1) R=R_{t-1}$. Therefore, we have $(t-1) R \cong R /\langle s-1\rangle \cong \mathbb{Z}[t] /\langle N(t)\rangle \cong \mathbb{Z}\left[\theta_{p}\right]$ and similarly for $(s-1) R$.

We will use this decomposition of $R$ into Dedekind rings to find a convenient characterization of projective $R$-modules.

Definition 12.23. An $R$-module $M$ is called exact if

$$
(t-1) M=M_{s-1} \text { and }(s-1) M=M_{t-1} .
$$

Note that $s(t-1)=(t-1)$ implies that $(t-1) M$ is a module over $R /\langle s-1\rangle \cong \mathbb{Z}\left[\theta_{p}\right]$.

Our aim is to show that a countably generated module over $R$ is projective if and only if it is an exact lattice. $R$ is an exact lattice and direct summands of exact lattices are exact lattices, this shows the "only if" part. The proof of the other direction follows ideas of [11] and also [8].

We begin with analyzing lattices over $R$, which are finitely generated. We will first show that a finitely generated, exact lattice $L$ with isomorphisms $(s-1) L \cong \mathbb{Z}\left[\theta_{p}\right]^{n}$ and $(t-1) L \cong \mathbb{Z}\left[\theta_{p}\right]^{n}$ is isomorphic to $R^{n}$. The structure of $L$ as it will be encountered is sufficiently complicated to be explained beforehand:

Let $c \in \mathbb{N}$, we construct an $R$-module $N_{c}$ as follows: Let $N_{c}$ be the free abelian group on generators $\left\{q_{0}, \ldots, q_{p-2}, r_{0}, \ldots r_{p-2}\right\}$. Define $s$ and $t$ on the $\mathbb{Z}$-basis $\left\{q_{0}, \ldots, q_{p-2}, r_{0}, \ldots r_{p-2}\right\}$ via

1. $t \cdot q_{i}:=q_{i}+c r_{0}$,

2. $t \cdot r_{i}:=r_{i+1}$ for $i<p-2$ and $t \cdot r_{p-2}:=-\sum_{k=0}^{p-2} r_{k}$,

3. $s \cdot q_{i}:=q_{i+1}$ for $i<p-2$ and $s \cdot q_{p-2}:=-c \sum_{k=0}^{p-2}(p-1-k) r_{k}-\sum_{k=0}^{p-2} q_{k}$,

4. $s \cdot r_{k}=r_{k}$.

Straightforward, but lengthy calculations show that $N_{c}$ is a well-defined $R$-module (Lemma 23.6 in the appendix). 
Lemma 12.24. Let $c \in \mathbb{N}, c \neq 0 \bmod p$, then $N_{c} \cong R$ as $R$-modules.

Proof. Since $c \neq 0 \bmod p$, there are $b \in \mathbb{N} \backslash\{0\}, a \in \mathbb{Z}$ such that $b c-1=a p$. Set $u:=\sum_{k=0}^{c-1} t^{k}, \gamma:=\sum_{k=0}^{c-2}(c-1-k) t^{k}$. A calculation shows that $u-c=(t-1) \gamma$. Define

$$
\Phi: N_{1} \rightarrow N_{c}, \quad \Phi\left(r_{i}\right):=u \cdot r_{i}, \Phi\left(q_{i}\right):=q_{i}+\gamma r_{0} .
$$

Straightforward calculations show that $\Phi$ commutes with $t$ and $s$. Therefore, it is a well-defined morphism of $R$-modules. Set $v:=\sum_{k=0}^{b-1} t^{c k}$, then $u \cdot v=1+a N(t)$. Since $N(t) \cdot r_{i}=0$ for all $i=0, \ldots, p-2$, we conclude $\Phi\left(v \cdot r_{i}\right)=v \cdot u \cdot r_{i}=r_{i}$. Hence $\Phi$ is surjective. Since both, $N_{c}$ and $N_{1}$, are free $\mathbb{Z}$-modules of the same finite rank we conclude that $\Phi$ is an isomorphism. Finally,

$$
\Theta: N_{1} \rightarrow R, \Theta\left(q_{i}\right):=s^{i}, \Theta\left(r_{i}\right):=t^{i}(t-1),
$$

defines an isomorphism of left $R$-modules.

Proposition 12.25. Let $L$ be an exact $R$-lattice such that there are $n \in \mathbb{N}$ and isomorphisms

$$
\phi_{s}:(s-1) L \cong \mathbb{Z}\left[\theta_{p}\right]^{n} \text { and } \phi_{t}:(t-1) L \cong \mathbb{Z}\left[\theta_{p}\right]^{n}
$$

of $\mathbb{Z}\left[\theta_{p}\right]$-modules, then $L \cong R^{n}$.

Proof. For $i=1, \ldots, n$, let $e_{i} \in \mathbb{Z}\left[\theta_{p}\right]^{n}$ be the column vector with 1 in the $i$ th summand and 0 elsewhere. Set

$$
b_{i}:=\phi_{t}^{-1}\left(e_{i}\right) \in(t-1) L
$$

Furthermore, choose $x_{1}, \ldots, x_{n} \in L$ such that $(s-1) x_{i}=\phi_{s}^{-1}\left(e_{i}\right)$. Let

$$
X:=\left\langle\left\{s^{j} x_{i} \mid 0 \leq j \leq p-2,1 \leq i \leq n\right\}\right\rangle_{\mathbb{Z}} \subseteq L
$$

be the $\mathbb{Z}$-submodule of $L$ which is generated by $\left\{s^{j} x_{i} \mid 0 \leq j \leq p-2,1 \leq i \leq n\right\}$. Since $\left\{\left(\theta_{p}\right)^{j} \cdot e_{i} \mid 0 \leq j \leq p-2,1 \leq i \leq n\right\}$ is a basis of $\mathbb{Z}\left[\theta_{p}\right]^{n}$ as a $\mathbb{Z}$-module, the restricted multiplication $(s-1)_{\mid X}: X \rightarrow(s-1) L$ is an isomorphism. Since $L$ is exact, we have $L=(t-1) L \oplus X$ as $\mathbb{Z}$-modules.

Set

$$
Q:=(t-1) L /(t-1)^{2} L
$$

and let $\pi:(t-1) L \rightarrow Q$ denote the quotient map. Then there is an isomorphism

$$
q:(\mathbb{Z} / p)^{n} \cong \mathbb{Z}\left[\theta_{p}\right]^{n} /\left(1-\theta_{p}\right) \mathbb{Z}\left[\theta_{p}\right]^{n} \cong Q
$$

Define

$$
\Psi: L \rightarrow Q, \quad x \rightarrow(1-t) x+(1-t)^{2} L, \text { and } \psi:=\Psi_{\mid X} .
$$

Since $(s-1)(t-1)=0, \psi: X \rightarrow Q$ is surjective. Therefore, $\left\{\psi\left(s^{j} x_{i}\right) \mid 0 \leq j \leq p-2,1 \leq\right.$ $i \leq n\}$ generates $Q$. Since $s(1-t)=(1-t),\left\{\psi\left(x_{i}\right) \mid 1 \leq i \leq n\right\}$ already generates $Q$. Since $\operatorname{dim}_{\mathbb{Z} / p}(Q)=n,\left\{\psi\left(x_{i}\right) \mid 1 \leq i \leq n\right\}$ is a basis of $Q .\left\{\pi\left(b_{i}\right) \mid 1 \leq i \leq n\right\}$ is also a 
basis of the $\mathbb{Z} / p$-vector space $Q$, namely, the one that corresponds to the standard basis under the isomorphism $q:(\mathbb{Z} / p)^{n} \cong Q$.

By regarding $\left(q^{-1} \psi\left(x_{1}\right), \ldots, q^{-1} \psi\left(x_{n}\right)\right)^{T}$ as a matrix in $G L_{n}(\mathbb{Z} / p)$, Lemma 12.6 provides us with $S=\left(s_{i j}\right)_{i, j=1, \ldots, n} \in G L_{n}(\mathbb{Z})$ and $c_{1}, \ldots, c_{n} \in \mathbb{N}, c_{i} \neq 0 \bmod p$ such that

$$
S\left(q^{-1} \circ \psi\left(x_{1}\right), \ldots, q^{-1} \psi\left(x_{n}\right)\right)=\operatorname{diag}\left(\left[c_{1}\right], \ldots,\left[c_{n}\right]\right) \in G L_{n}(\mathbb{Z} / p) .
$$

This implies $\psi\left(\sum_{k=1}^{n} s_{k j} x_{k}\right)=c_{j} \pi\left(b_{j}\right)$ for $j=1, \ldots, n$. Define an automorphism of $\mathbb{Z}\left[\theta_{p}\right]^{n}$ by

$$
S: \mathbb{Z}\left[\theta_{p}\right]^{n} \rightarrow \mathbb{Z}\left[\theta_{p}\right]^{n}, \quad\left(\xi_{1}, \ldots, \xi_{n}\right) \mapsto\left(\sum_{k=1}^{n} s_{k 1} \xi_{k}, \ldots, \sum_{k=1}^{n} s_{k n} \xi_{k}\right) .
$$

Then, by replacing $\phi_{s}$ by $\phi_{s} \circ S^{-1}$ and $x_{i}$ by $\sum_{k=1}^{n} s_{k j} x_{k}$, we may assume that

$$
\exists c_{1}, \ldots, c_{n} \in \mathbb{N},\left[c_{i}\right] \neq 0 \in \mathbb{Z} / p \text { such that } \psi\left(x_{i}\right)=c_{i} \pi\left(b_{i}\right) .
$$

Therefore, there are $u_{i} \in(t-1) L$ such that

$$
(t-1) x_{i}=c_{i} b_{i}+(t-1) u_{i} \text { for } i=1, \ldots, n .
$$

Set

$$
y_{i}:=x_{i}-u_{i}, \text { then } t y_{i}=c_{i} b_{i}+y_{i} \text { for } i=1, \ldots, n \text {. }
$$

Let

$$
Y:=\left\langle\left\{s^{j} \cdot y_{i} \mid 0 \leq j \leq p-2,1 \leq i \leq n\right\}\right\rangle_{\mathbb{Z}} \subseteq L .
$$

Since $(s-1) x_{i}=(s-1) y_{i}$, there is a direct sum decomposition

$$
L \cong(t-1) L \oplus_{\mathbb{Z}} Y
$$

as $\mathbb{Z}$-modules. Furthermore, $\left\{s^{j} y_{i} \mid 0 \leq j \leq p-2,1 \leq i \leq n\right\}$ is a basis of $Y$ as a free $\mathbb{Z}$-module and $\left\{t^{j} b_{i} \mid 0 \leq j \leq p-2,1 \leq i \leq n\right\}$ is a basis of $(t-1) L$ as a free $\mathbb{Z}$-module. Set

$$
L_{i}:=\left\langle\left\{t^{j} b_{i}, s^{j} y_{i} \mid 0 \leq j \leq p-2\right\}\right\rangle_{\mathbb{Z}}, i=1, \ldots, n .
$$

Note that $\left\{t^{j} b_{i}, s^{j} y_{i} \mid 0 \leq j \leq p-2,\right\}$ is a free $\mathbb{Z}$-basis of $L_{i}$.

Claim 12.26. $L_{i}$ is an $R$-submodule of $L$ and $L_{i} \cong N_{c_{i}}$ as $R$-modules.

Define an isomorphism of abelian groups $\phi: L_{i} \cong N_{c_{i}}$ by sending $t^{j} b_{i}$ to $r_{j}$ and $s^{j} y_{i}$ to $q_{j}$ for $j=0, \ldots, p-2$. The following calculations show that $L_{i}$ is closed under multiplication by $t$ and $s$ (hence an $R$-submodule) and that $\phi$ is an isomorphism of $R$-modules: Since $b_{i} \in(t-1) L$, we have $s b_{i}=b_{i}$ and $N(s) b_{i}=p b_{i}$. Therefore,

$$
t^{p-1} b_{i}=p b_{i}-N(s) b_{i}-\left(N(t)-t^{p-1}\right) b_{i}=-\sum_{k=0}^{p-2} t^{k} \cdot b_{i}
$$

and

$$
t s^{j} y_{i}=s^{j} y_{i}+c_{i} b_{i}
$$


Another straightforward calculation yields $N(t) y_{i}=p y_{i}+\sum_{k=0}^{p-2}(p-1-k) t^{k} c_{i} b_{i}$. Therefore,

$$
s^{p-1} y_{i}=p y_{i}-N(t) y_{i}-\left(N(s)-s^{p-1}\right) y_{i}=-\sum_{k=0}^{p-2}(p-1-k) t^{k} c_{i} b_{i}-\sum_{k=0}^{p-2} s^{k} y_{i}
$$

and also

$$
s t^{j} b_{i}=t^{j} b_{i}
$$

By construction, $L \cong \bigoplus_{i=1}^{n} L_{i}$ as abelian groups. By the last claim, $L=\bigoplus_{i=1}^{n} L_{i} \cong$ $\bigoplus_{i=1}^{n} N_{c_{i}}$ as $R$-modules. This implies that $L \cong R^{n}$ by Lemma 12.24 .

Proposition 12.27. Let $L$ be a finitely generated, exact lattice over $R$, then $L$ is projective.

Proof. We will show that $L$ is a direct summand of $R^{n}$ for some $n \in \mathbb{N}$. $(t-1) L$ and $(s-1) L$ are lattices over $\mathbb{Z}\left[\theta_{p}\right]$ via the identifications $R /\langle s-1\rangle \cong \mathbb{Z}\left[\theta_{p}\right]$ and $R /\langle t-1\rangle \cong$ $\mathbb{Z}\left[\theta_{p}\right]$. By considering $L \oplus \gamma^{*}(L)$ instead of $L$, we may assume that $(t-1) L \cong(s-1) L$ as $\mathbb{Z}\left[\theta_{p}\right]$-modules (recall that $\gamma$ is the involution on $R$ that interchanges $s$ and $t$ ).

We will now construct a finitely generated, exact $R$-lattice $L^{\prime}$ such that there are isomorphisms $(t-1)\left(L \oplus L^{\prime}\right) \cong \mathbb{Z}\left[\theta_{p}\right]^{n} \cong(s-1)\left(L \oplus L^{\prime}\right)$ as $\mathbb{Z}\left[\theta_{p}\right]$-modules. By Proposition 12.25 , this will show the claim.

Define an $R-\mathbb{Z}\left[\theta_{p}\right]$-bimodule $\Lambda$ by defining a right action of $\mathbb{Z}\left[\theta_{p}\right]$ on $R$ via the ring homomorphism:

$$
\lambda: \mathbb{Z}\left[\theta_{p}\right] \hookrightarrow R, \quad \theta_{p} \mapsto s+t-1
$$

To check that $\lambda$ is well-defined compute $(s+t-1)^{k}=s^{k}+t^{k}-1$ for all $k \in \mathbb{N}$. This shows that $N(s+t-1)=N(t)+N(s)-p=0$. It is also straightforward to check that $(1-t) \Lambda$ and $(1-s) \Lambda$ are isomorphic to $\mathbb{Z}\left[\theta_{p}\right]$ as $\mathbb{Z}\left[\theta_{p}\right]$-bimodules (Recall that every left $R$-module $M$ gives rise to left $\mathbb{Z}\left[\theta_{p}\right]$-modules $(1-t) M$ and $\left.(1-s) M\right)$. Since $\Lambda \otimes_{\mathbb{Z}\left[\theta_{p}\right]} \mathbb{Z}\left[\theta_{p}\right] \cong R$ as left $R$-modules we obtain an additive functor

$$
\Lambda \otimes_{\mathbb{Z}\left[\theta_{p}\right]}\left(\_\right): \mathfrak{M o d}\left(\mathbb{Z}\left[\theta_{p}\right]\right) \rightarrow \mathfrak{M o d}(R),
$$

which maps projectives to projectives. Since $(t-1) L \cong(s-1) L$ are $\mathbb{Z}\left[\theta_{p}\right]$-lattices, there is a $\mathbb{Z}\left[\theta_{p}\right]$-module $P$ such that $(t-1) L \oplus P \cong(s-1) L \oplus P \cong \mathbb{Z}\left[\theta_{p}\right]^{n}$. This shows that $(t-1)\left(L \oplus \Lambda \otimes_{\mathbb{Z}\left[\theta_{p}\right]} P\right) \cong(s-1)\left(L \oplus \Lambda \otimes_{\mathbb{Z}\left[\theta_{p}\right]} P\right) \cong \mathbb{Z}\left[\theta_{p}\right]^{n}$. Therefore, $L \oplus \Lambda \otimes_{\mathbb{Z}\left[\theta_{p}\right]} P \cong R^{n}$ by Proposition 12.25 .

Lemma 12.28. Let $L$ be an $R$-lattice of $\mathbb{Z}$-rank $\leq 2 p-2$, then either $L$ is exact or there is a decomposition $L \cong L_{t-1} \oplus L_{s-1}$.

Proof. If $(t-1) L=\{0\}$ or $(s-1) L=\{0\}$, then $L \cong L_{t-1} \oplus L_{s-1}$ holds. So we may assume that $(t-1) L$ and $(s-1) L$ are non-zero. As $\mathbb{Z}\left[\theta_{p}\right]$-lattices they decompose into fractional ideals by Theorem 12.10. Since both are non-zero, they have $\mathbb{Z}$-rank at least $p-1$ by Lemma 12.13 , since $L_{t-1} \cap L_{s-1}=\{0\}$ their $\mathbb{Z}$-rank is exactly $p-1$, so there are fractional ideals $\mathfrak{A}$ and $\mathfrak{B}$ such that $L_{t-1} \cong \mathfrak{A}$ and $L_{s-1} \cong \mathfrak{B}$ as $\mathbb{Z}\left[\theta_{p}\right]$-modules. If 
$L$ is not exact, then $(t-1) L \neq L_{s-1}$ or $(s-1) L \neq L_{t-1}$. Let us first assume that $(t-1) L \neq L_{s-1}$ :

Since $(t-1) L_{s-1} \subseteq(t-1) L \subseteq L_{s-1}$ and $L_{s-1} /(t-1) L_{s-1} \cong \mathfrak{B} /\left(\theta_{p}-1\right) \mathfrak{B} \cong \mathbb{Z} / p$, $(t-1) L=(t-1) L_{s-1}$. Write $L=L_{s-1} \oplus_{\mathbb{Z}} X$, where $X$ is a free $\mathbb{Z}$-submodule of $L$ such that multiplication by $s-1$ induces an isomorphism $(s-1)_{\mid X}: X \rightarrow(s-1) L$. Since $L_{s-1} \cong \mathfrak{B}$, multiplication by $(t-1)$ induces an isomorphism $(t-1): L_{s-1} \rightarrow(t-1) L_{s-1}$. Let $\phi$ be the composition

$$
\phi: X \stackrel{(t-1)}{\longrightarrow}(t-1) L=(t-1) L_{s-1} \stackrel{(t-1)^{-1}}{\longrightarrow} L_{s-1} \hookrightarrow L .
$$

Set $Y:=($ id $-\phi)(X)$. Since $\phi(X) \subset L_{s-1}$, we still have a decomposition $L=L_{s-1} \oplus_{\mathbb{Z}} Y$. We will show that $Y$ is equal to $L_{t-1}$. Let $x \in X$. Set $z:=\phi(x)$, i.e. then $(t-1) x=(t-1) z$ and $t(x-\phi(x))=t(x-z)=(x-z)=x-\phi(x)$, hence $t_{\mid Y}=\operatorname{id}_{\mid Y}$. Therefore, $Y \subseteq L_{t-1}$. If $x \in L_{t-1}, x=z+y$ with $z \in L_{s-1}$ and $y \in Y$, then $(t-1) x=(t-1) z$, which shows that $z \in L_{s-1} \cap L_{t-1}=\{0\}$. Hence $L_{t-1} \subseteq Y$. Therefore, $L \cong L_{s-1} \oplus L_{t-1}$.

If $(s-1) L \neq L_{t-1}$ then we can apply the argument above to $\gamma^{*} L$ to see that $L \cong$ $L_{s-1} \oplus L_{t-1}$. Hence we have shown that if $L$ is not exact then there is a decomposition $L \cong L_{s-1} \oplus L_{t-1}$. This shows the lemma.

Proposition 12.29. Let $L$ be an exact $R$-lattice, and $x$ in $L$, then there is a finitely generated, exact $R$-sublattice $L^{\prime} \subseteq L$ such that $x \in L$ and $L / L^{\prime}$ is an exact $R$-lattice.

Proof. Let us first show that $\bigcap_{n \in \mathbb{N}}(t-1)^{n} L=\{0\}$. Note that $(t-1) L=L_{s-1}$ is a $\mathbb{Z}\left[\theta_{p}\right]$-lattice and therefore, by Theorem 12.14 , projective. Hence it suffices to show that $\bigcap_{n \in \mathbb{N}}(t-1)^{n} \mathbb{Z}\left[\theta_{p}\right]=\{0\}$. Assume $y \in \bigcap_{n \in \mathbb{N}}\left\langle\theta_{p}-1\right\rangle^{n}$ were non-zero then $\langle y\rangle$ would have a unique factorization into a (finite) product of prime ideals. But since $\left\langle\theta_{p}-1\right\rangle^{n} \subseteq\langle y\rangle$ for all $n \in \mathbb{N}$, Lemma 12.8 yields a contradiction.

Case 1: $x \in L_{s-1}$. Since $\bigcap_{n \in \mathbb{N}}(t-1)^{n} L=\{0\}$, there is $x^{\prime} \in L$ such that $x^{\prime} \notin(t-1) L=$ $L_{s-1}$ and $x=(t-1)^{k} x^{\prime}$. Therefore, we may assume that $x \notin(t-1) L_{s-1}$. Let $y \in L \backslash L_{s-1}$ such that $x=(t-1) y$ and let $L^{\prime}$ be the pure closure of $R y$, i.e.,

$$
L^{\prime}:=\{z \in L \mid \exists n \in \mathbb{Z}: n z \in R y\} .
$$

$L^{\prime}$ is obviously an $R$-module. Since $L$ is a lattice there is an index set $I$ and $\mathbb{Z}$-isomorphism $\phi: L \cong \bigoplus_{I} \mathbb{Z}$, therefore, there is a subset $I_{0} \subseteq I$ such that $\phi(R y) \subseteq \bigoplus_{I_{0}} \mathbb{Z}$. Since $R$ is free of rank $2 p-2$ as an abelian group, we may, after a base change, assume that $\left|I_{0}\right| \leq 2 p-2$. This shows that $L^{\prime}$ has $\mathbb{Z}$-rank $\leq 2 p-2 . L^{\prime}$ cannot have a decomposition $L^{\prime}=L_{s-1}^{\prime} \oplus L_{t-1}^{\prime}$, because this would imply $x \in(t-1) L_{s-1}^{\prime} \subseteq(t-1) L_{s-1}$. Hence, by Lemma $12.28, L^{\prime}$ is exact.

Since $L^{\prime}$ is pure, a base change in $\bigoplus_{I_{0}} \mathbb{Z}$ shows that $L / L^{\prime}$ is a lattice as well. The long exact sequence of a short exact sequence of chain complexes shows that $L / L^{\prime}$ is exact.

Case 2: $x \in L_{t-1}$. Apply the involution $\gamma$, which interchanges $t$ and $s$.

Case 3: $x \notin L_{s-1}, x \notin L_{t-1}$. Apply Case 1 to $(t-1) x \in L_{s-1}$ to see that there is a finitely generated, exact sublattice $L_{1} \subseteq L$ such that $(t-1) x \in L_{1}$ and $L / L_{1}$ is an exact lattice. Let $\pi_{1}$ be the projection from $L$ onto $L / L_{1}$. By Case 2, there 
is a finitely generated, exact sublattice $L_{2} \subseteq L / L_{1}$ such that $(s-1) \pi(x) \in L_{2}$ and $\left(L / L_{1}\right) / L_{2}$ is a lattice. Let $\pi_{2}$ be the projection from $L / L_{1}$ onto $\left(L / L_{1}\right) / L_{2}$. We have $(s-1) \pi_{2} \pi_{1}(x)=(t-1) \pi_{2} \pi_{1}(x)=0$, hence $\pi_{2} \pi_{1}(x)=0$ by Lemma 12.21. Thus $x \in \pi_{1}^{-1}\left(L_{2}\right)$. The restriction of $\pi_{1}$ to $\pi_{1}^{-1}\left(L_{2}\right)$ yields an exact sequence of $R$-lattices $L_{1} \longmapsto \pi_{1}^{-1}\left(L_{2}\right) \rightarrow L_{2}$. The long exact sequence in homology again shows that $\pi_{1}^{-1}\left(L_{2}\right)$ is an exact $R$-lattice, moreover $L / \pi_{1}^{-1}\left(L_{2}\right) \cong\left(L / L_{1}\right) / L_{2}$ is a lattice and of course $\pi_{1}^{-1}\left(L_{2}\right)$ is finitely generated. Therefore, setting $L^{\prime}:=\pi_{1}^{-1}\left(L_{2}\right)$ yields the claim.

Theorem 12.30. Let $M$ be a countably generated $R$-module, then $M$ is projective if and only if it is an exact $R$-lattice.

Proof. We only have to show the "if" direction. Assume that $M$ is a countably generated, exact $R$-lattice and let $\left(x_{n}\right)_{n \in \mathbb{N}}$ be a sequence of generators. We will define inductively a sequence of finitely generated, exact $R$-sublattices $\left(M_{n}\right)_{n \in \mathbb{N}}$ of $M$ such that $M_{n} \subseteq M_{n+1}$, $x_{n} \in M_{n}$ and $M / M_{n}$ is an exact lattice. Use Proposition 12.29 to choose a finitely generated, exact $R$-lattice $M_{0}$ such that $x_{0} \in M_{0}$ and $M / M_{0}$ is an exact $R$-lattice. Assume that $M_{0}, \ldots, M_{n}$ as above have been constructed, let $\pi_{n}$ be the projection from $M$ onto $M / M_{n}$. Again by Proposition 12.29 , there is a finitely generated, exact $R$-lattice $P_{n+1} \subseteq M / M_{n+1}$ such that $\pi_{n}\left(x_{n+1}\right) \in P_{n+1}$ and $\left(M / M_{n}\right) / P_{n+1}$ is an exact $R$-lattice. $M_{n+1}:=\pi_{n}^{-1}\left(P_{n+1}\right)$ has the desired properties.

Define $P_{0}:=M_{0}$, we have $P_{n} \cong M_{n} / M_{n-1}$ for $n \geq 1$. By Proposition 12.27 , all $P_{n}$ are projective $R$-modules. For every $n \in \mathbb{N}$, there is an exact sequence

$$
M_{n} \longmapsto M_{n+1} \rightarrow P_{n} .
$$

By induction, there are isomorphisms $\phi_{n}: M_{n} \cong \bigoplus_{i=0}^{n} P_{i}$ such that

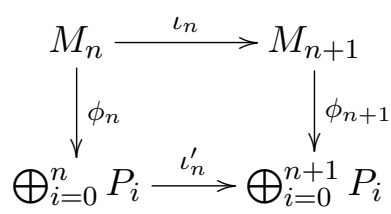

commutes (here $\iota_{n}$ and $\iota_{n}^{\prime}$ denote the obvious inclusions). Since $M=\bigcup_{n \in \mathbb{N}} M_{n}$, this shows that

$$
M \cong \bigoplus_{n \in \mathbb{N}} P_{n}
$$

is projective.

\subsection{Lattices over $\mathcal{R}$ and the Proof of Theorem 12.1}

Let us first explain how to induce $\mathcal{R}$-modules from modules over one of the subrings ${ }_{i} \mathcal{R}_{i}$, $i \in\{0,1,2\}$.

Let $M$ be an ${ }_{i} \mathcal{R}_{i}$-module and set $\operatorname{Ind}_{i} M:=\mathcal{R}_{i} \otimes_{i} \mathcal{R}_{i} M$. Using ${ }_{i} \mathcal{R} \otimes_{\mathcal{R}} \mathcal{R}_{i}={ }_{i} \mathcal{R}_{i}$, we see that ${ }_{i}\left(\operatorname{Ind}_{i} M\right)=M$. 
The easiest example of an induced $\mathcal{R}$-module is given by $\mathcal{R}_{i}=\operatorname{Ind}_{i i} \mathcal{R}_{i}, i=0,1,2$. Note that these modules are projective and that for every $\mathcal{R}$-module $M$,

$$
\operatorname{Hom}_{\mathcal{R}}\left(\mathcal{R}_{i}, M\right) \rightarrow{ }_{i} M, \phi \mapsto \phi\left({ }_{i} 1_{i}\right)
$$

is an isomorphism of ${ }_{i} \mathcal{R}_{i}$-modules.

$\operatorname{Ind}_{i}$ is left adjoint to the restriction functor $M \mapsto{ }_{i} M$, i.e., for an ${ }_{i} \mathcal{R}_{i}$-module $M$ and an $\mathcal{R}$-module $N$, there is a natural isomorphism

$$
\operatorname{Hom}_{\mathcal{R}}\left(\operatorname{Ind}_{i} M, N\right) \cong \operatorname{Hom}_{i} \mathcal{R}_{i}\left(M, \operatorname{Hom}_{i} \mathcal{R}_{i}\left(\mathcal{R}_{i}, N\right)\right) \cong \operatorname{Hom}_{i} \mathcal{R}_{i}\left(M,{ }_{i} N\right) .
$$

Lemma 12.31. Let $P$ be a projective ${ }_{i} \mathcal{R}_{i}$-module. Then $\operatorname{Ind}_{i} P$ is projective.

Proof. There is $Q$ and an index set $I$ such that $P \oplus Q \cong \bigoplus_{I}{ }_{i} \mathcal{R}_{i}$. Since $\mathcal{R}_{i}=\operatorname{Ind}_{i i} \mathcal{R}_{i}$ and $\mathcal{R}_{i}$ is projective, this shows that $\operatorname{Ind}_{i} P$ is projective.

Note that this implies that $\operatorname{Ind}_{i} P$ is an exact $\mathcal{R}$-lattice. Recall that we want to show that every exact countably generated $\mathcal{R}$-lattice is projective. We will do this by decomposing a countably generated $\mathcal{R}$-lattice $L$ into a direct sum of lattices, which are induced from projective lattices over the ${ }_{i} \mathcal{R}_{i}$.

Theorem 12.32. Let $L$ be a countably generated, exact $\mathcal{R}$-lattice, then $L$ is projective.

Proof. ${ }_{0} L$ is a ${ }_{0} \mathcal{R}_{0}$-lattice. By Theorem 12.20 , there are ${ }_{0} \mathcal{R}_{0}$-sublattices ${ }_{0} L_{1},{ }_{0} L_{2}$ and ${ }_{0} L_{3}$ such that ${ }_{0} L={ }_{0} L_{1} \oplus_{0} L_{2} \oplus_{0} L_{3}$ and

1. ${ }_{0} L_{1} \subseteq\left({ }_{0} L\right)_{0} t_{0}-1 \subseteq{ }_{0} L_{1} \oplus_{0} L_{3}$;

2. ${ }_{0} L_{2} \subseteq\left({ }_{0} L\right)_{N\left({ }_{0} t_{0}\right)} \subseteq{ }_{0} L_{2} \oplus_{0} L_{3}$;

3. ${ }_{0} L_{3}$ is projective and exact.

Let $P_{0}$ be the sublattice of $L$ generated by ${ }_{0} L_{3}$, i.e.,

$$
P_{0}:=\mathcal{R}_{0} \cdot{ }_{0} L_{3} \subseteq L
$$

Claim 12.33. $P_{0} \cong \operatorname{Ind}_{0}\left({ }_{0} L_{3}\right)$, in particular, $P_{0}$ is projective.

Set

$$
\Phi: \operatorname{Ind}_{0}\left({ }_{0} L_{3}\right)=\mathcal{R}_{0} \otimes_{0} \mathcal{R}_{0}{ }_{0} L_{3} \rightarrow \mathcal{R}_{0} \cdot{ }_{0} L_{3}, \quad r \otimes l \mapsto r \cdot l .
$$

$\Phi$ is surjective. Note that ${ }_{i} \operatorname{Ind}_{0}\left({ }_{0} L_{3}\right)={ }_{i} \mathcal{R}_{0} \otimes_{0} \mathcal{R}_{0}{ }_{0} L_{3}$. Let $\Phi_{i}:=\Phi_{\left.\right|_{i} \operatorname{Ind}_{0}\left({ }_{0} L_{3}\right)}$. It is clear that $\Phi_{0}$ is an isomorphism. Let $k \in\{1,2\}$ and $x \in{ }_{k} \operatorname{Ind}_{0}\left({ }_{0} L_{3}\right)$ such that $\Phi_{k}(x)=0$, since ${ }_{k} \mathcal{R}_{0}={ }_{k} \alpha_{0} \cdot{ }_{0} \mathcal{R}_{0}$, we can write $x={ }_{k} \alpha_{0} \otimes y$ for a $y \in{ }_{0} L_{3}$. Hence $\Phi_{k}(x)=0$ implies that ${ }_{k} \alpha_{0} \cdot y=0$ in $L$. Therefore, ${ }_{0} \alpha_{k} \cdot{ }_{k} \alpha_{0} y=0$ in $L_{3}$. Since $L_{3}$ is an exact ${ }_{0} \mathcal{R}_{0}$-module, ${ }_{0} \alpha_{1} \cdot{ }_{1} \alpha_{0}=N\left({ }_{0} t_{0}\right)$ and ${ }_{0} \alpha_{2} \cdot{ }_{2} \alpha_{0}={ }_{0} t_{0}-1$, there is $z \in L_{3}$ such that $y={ }_{0} \alpha_{l} \cdot{ }_{l} \alpha_{0} \cdot z$ for $l \in\{1,2\}, l \neq k$. But then

$$
x={ }_{k} \alpha_{0} \otimes y={ }_{k} \alpha_{0} \cdot{ }_{0} \alpha_{l} \cdot{ }_{l} \alpha_{0} \otimes z=0 .
$$

This shows that $\Phi_{k}$ is one-to-one and proves the claim. 
Claim 12.34. $L / P_{0}$ is an exact lattice.

Since $P_{0}$ is exact, we only have to show that $L / P_{0}$ is a lattice. Since ${ }_{i} P_{0}={ }_{i} \alpha_{0} \cdot{ }_{0} L_{3}$ for $i=1,2$, it suffices to show that ${ }_{i} L /{ }_{i} \alpha_{0} \cdot{ }_{0} L_{3}$ is a lattice for $i=1,2$. We will show it only for $i=2$, since the other case follows by exactly the same arguments. First observe that exactness of $L$ implies that ${ }_{2} L \cong \mathrm{im}_{2} \alpha_{0} \oplus_{\mathbb{Z}} \operatorname{im}_{1} \alpha_{2}$ decomposes as a direct sum of $\mathbb{Z}$-lattices. Let us show that $\operatorname{im}_{2} \alpha_{0}={ }_{2} \alpha_{0} \cdot{ }_{0} L_{2} \oplus_{2} \alpha_{0} \cdot{ }_{0} L_{3}$ since this implies that ${ }_{2} L /{ }_{2} \alpha_{0} \cdot{ }_{0} L_{3}$ is a lattice. Let $x \in{ }_{0} L_{1}$, since ${ }_{0} L_{1} \subseteq\left({ }_{0} L\right)_{0} t_{0}-1,{ }_{0} \alpha_{2} \cdot{ }_{2} \alpha_{0} x=\left({ }_{0} t_{0}-1\right) x=0$. By exactness of $L$, there is $y \in{ }_{1} L$ such that ${ }_{2} \alpha_{0} x={ }_{2} \alpha_{1} y$, therefore, $\left({ }_{2} t_{2}-1\right)_{2} \alpha_{0} x=$ $\left({ }_{2} s_{2}-1\right)_{2} \alpha_{0} x=0$. By Lemma 12.21, ${ }_{2} \alpha_{0} x=0$. This shows ${ }_{2} \alpha_{0} \cdot{ }_{0} L_{1}=\{0\}$. Hence $\operatorname{im}_{2} \alpha_{0}={ }_{2} \alpha_{0} \cdot{ }_{0} L_{2}+{ }_{2} \alpha_{0} \cdot{ }_{0} L_{3}$. Now assume that there is a $x \in{ }_{2} \alpha_{0} \cdot{ }_{0} L_{2} \cap_{2} \alpha_{0} \cdot{ }_{0} L_{3}$. Then there are $y_{2} \in{ }_{0} L_{2}$ and $y_{3} \in{ }_{0} L_{3}$ such that $x={ }_{2} \alpha_{0} \cdot y_{2}={ }_{2} \alpha_{0} \cdot y_{3}$. Since ${ }_{2} \alpha_{0} \cdot\left(y_{2}-y_{3}\right)=0$, there is $z \in{ }_{1} L$ such that ${ }_{2} \alpha_{1} z=y_{2}-y_{3}$. But im ${ }_{2} \alpha_{1} \subseteq\left({ }_{0} L\right)_{0} t_{0}-1 \subseteq{ }_{0} L_{1} \oplus_{0} L_{3}$, this shows that $y_{2}=0$. Therefore, $x={ }_{2} \alpha_{0} \cdot y_{2}=0$. Hence $\operatorname{im}_{2} \alpha_{0}={ }_{2} \alpha_{0} \cdot{ }_{0} L_{2} \oplus{ }_{2} \alpha_{0} \cdot{ }_{0} L_{3}$. This finishes the proof of the claim

Set

$$
L^{\prime}:=L / P_{0}
$$

$L^{\prime}$ is an exact $\mathcal{R}$-lattice such that ${ }_{0} L^{\prime} \cong{ }_{0} L_{1} \oplus_{0} L_{2}$ (Note that $\left.{ }_{0} L^{\prime}={ }_{0} L /{ }_{0} L_{3}\right)$. This implies that ${ }_{0} L^{\prime}={ }_{0} L_{N\left({ }_{0} t_{0}\right)}^{\prime} \oplus{ }_{0} L_{\left(0 t_{0}-1\right)}^{\prime}$. Again by Theorem 12.20 , there are ${ }_{1} \mathcal{R}_{1}$-sublattices ${ }_{1} L_{1}^{\prime},{ }_{1} L_{2}^{\prime}$ and ${ }_{1} L_{3}^{\prime}$ of ${ }_{1} L^{\prime}$ such that ${ }_{1} L^{\prime}={ }_{1} L_{1}^{\prime} \oplus_{1} L_{2}^{\prime} \oplus_{1} L_{3}^{\prime}$ and

1. ${ }_{1} L_{1}^{\prime} \subseteq\left({ }_{1} L^{\prime}\right)_{1 s_{1}-1} \subseteq{ }_{1} L_{1}^{\prime} \oplus{ }_{1} L_{3}^{\prime}$;

2. ${ }_{1} L_{2}^{\prime} \subseteq\left({ }_{1} L^{\prime}\right){ }_{N\left({ }_{1} s_{1}\right)} \subseteq{ }_{1} L_{2}^{\prime} \oplus{ }_{1} L_{3}^{\prime}$;

3. ${ }_{1} L_{3}^{\prime}$ is projective and exact.

Set

$$
P_{1}:=\mathcal{R}_{1} \cdot{ }_{1} L_{3}^{\prime} .
$$

By exactly the same arguments as above, $P_{1}$ is a projective $\mathcal{R}$-module. The quotient

$$
L^{\prime \prime}:=L^{\prime} / P_{2}
$$

is an exact $\mathcal{R}$-lattice such that

$$
{ }_{1} L^{\prime \prime}={ }_{1} L_{N\left({ }_{1} s_{1}\right)}^{\prime \prime} \oplus{ }_{1} L_{\left({ }_{1} s_{1}-1\right)}^{\prime \prime}
$$

Since ${ }_{0} P_{1}={ }_{0} \alpha_{11} L_{3}^{\prime} \subseteq{ }_{0} L_{\left({ }_{0} t_{0}-1\right)}^{\prime}$, we also have

$$
{ }_{0} L^{\prime \prime}={ }_{0} L_{N\left({ }_{0} t_{0}\right)}^{\prime \prime} \oplus{ }_{0} L_{\left({ }_{0} t_{0}-1\right)}^{\prime \prime} .
$$

If $x \in{ }_{0} L_{\left({ }_{0} t_{0}-1\right)}^{\prime \prime}$ and ${ }_{1} \alpha_{0} \cdot x=0$, then $0=N\left({ }_{0} t_{0}\right) x=p x$. This implies $x=0$, hence $\left({ }_{1} \alpha_{0}\right)_{\left.\left.\right|_{0} L_{(0} t_{0}-1\right)}^{\prime \prime}$ is one-to-one. If $y \in{ }_{1} L_{\left(1 s_{1}-1\right)}^{\prime \prime}$, then ${ }_{2} \alpha_{1} \cdot y \in{ }_{2} L_{\left(2 t_{2}-1\right)}^{\prime \prime} \cap_{2} L_{\left(2 s_{2}-1\right)}^{\prime \prime}=\{0\}$ hence $y \in \operatorname{im}_{1} \alpha_{0}$. On the other hand $\operatorname{im}_{1} \alpha_{0}$ is clearly contained in ${ }_{1} L_{\left(1 s_{1}-1\right)}^{\prime \prime}$. This shows that

$$
\left({ }_{1} \alpha_{0}\right)_{\left.\left.\right|_{0} L_{(0} t_{0}-1\right)}:{ }_{0} L_{\left({ }_{0} t_{0}-1\right)}^{\prime \prime} \rightarrow{ }_{1} L_{\left(1 s_{1}-1\right)}^{\prime \prime}
$$


is an isomorphism. By exactly the same arguments,

$$
\left({ }_{0} \alpha_{1}\right)_{\mid{ }_{1} L_{\left(1 s_{1}-1\right)}^{\prime \prime}}:{ }_{1} L_{\left(1 s_{1}-1\right)}^{\prime \prime} \rightarrow{ }_{0} L_{\left({ }_{0} t_{0}-1\right)}^{\prime \prime}
$$

is an isomorphism as well. Therefore, multiplication by ${ }_{0} \alpha_{11} \alpha_{0}=N\left({ }_{0} t_{0}\right)$ is an automorphism of ${ }_{0} L_{\left({ }_{0} t_{0}-1\right)}^{\prime \prime}$. But $N\left({ }_{0} t_{0}\right)_{\left.\left.\right|_{0} L_{(0} t_{0}-1\right)}^{\prime \prime}$ is just multiplication by $p$. Therefore, ${ }_{0} L_{\left(0, t_{0}-1\right)}^{\prime \prime}={ }_{1} L_{\left({ }_{1} s_{1}-1\right)}^{\prime \prime}=\{0\}$.

Claim 12.35. ${ }_{1} \alpha_{0} \cdot{ }_{0} L^{\prime \prime}={ }_{0} \alpha_{1} \cdot{ }_{1} L^{\prime \prime}=\{0\}$.

We have ${ }_{1} \alpha_{0} \cdot{ }_{0} t_{0}={ }_{1} \alpha_{0}$. Now let $x \in{ }_{0} L_{N\left({ }_{0} t_{0}\right)}$, then $p \cdot{ }_{1} \alpha_{0} \cdot x={ }_{1} \alpha_{0} \cdot N\left({ }_{0} t_{0}\right) x=0$, hence ${ }_{1} \alpha_{0} \cdot x=0$, this shows that ${ }_{1} \alpha_{0} \cdot{ }_{0} L_{N\left({ }_{0} t_{0}\right)}^{\prime \prime}=0$. Similarly ${ }_{0} \alpha_{1} \cdot{ }_{1} L_{N\left({ }_{1} s_{1}\right)}^{\prime \prime}=\{0\}$. Since ${ }_{1} L^{\prime \prime}={ }_{1} L_{N\left(s_{1}\right)}^{\prime \prime}$ and ${ }_{0} L^{\prime \prime}={ }_{0} L_{N\left({ }_{0} t_{0}\right)}^{\prime \prime}$, this implies the claim.

Claim 12.36. $L^{\prime \prime}$ is projective.

By the previous claim and exactness of $L,{ }_{2} \alpha_{1}:{ }_{1} L^{\prime \prime} \rightarrow{ }_{2} L^{\prime \prime}$ and ${ }_{2} \alpha_{0}:{ }_{0} L^{\prime \prime} \rightarrow{ }_{2} L^{\prime \prime}$ are one-to-one and ${ }_{1} \alpha_{2}:{ }_{2} L^{\prime \prime} \rightarrow{ }_{1} L^{\prime \prime}$ and ${ }_{0} \alpha_{2}:{ }_{2} L^{\prime \prime} \rightarrow{ }_{0} L^{\prime \prime}$ are onto. Since ${ }_{2} t_{2}-1={ }_{2} \alpha_{0} \cdot{ }_{0} \alpha_{2}$ and ${ }_{2} s_{2}-1={ }_{2} \alpha_{1} \cdot{ }_{1} \alpha_{2}$, this implies that ${ }_{2} L^{\prime \prime}$ is an exact ${ }_{2} \mathcal{R}_{2}$-lattice. By Theorem 12.30, ${ }_{2} L^{\prime \prime}$ is a projective ${ }_{2} \mathcal{R}_{2}$-module. Hence $\operatorname{Ind}_{2}\left({ }_{2} L^{\prime \prime}\right)$ is a projective $\mathcal{R}$-module. Note that $\mathcal{R}_{22} \cdot L^{\prime \prime}=L^{\prime \prime}$. Set

$$
\Phi: \operatorname{Ind}_{2}\left({ }_{2} L^{\prime \prime}\right) \rightarrow L^{\prime \prime}, \quad r \otimes x \mapsto r x .
$$

By exactly the same arguments as in the proof of Claim \#1, we see that $\phi$ is an isomorphism, this proves Claim \#4.

So far we have two short exact sequences of $\mathcal{R}$-modules, namely

$$
P_{0} \longmapsto L \rightarrow L^{\prime}
$$

and

$$
P_{1} \longmapsto L^{\prime} \rightarrow L^{\prime \prime}
$$

with $P_{0}, P_{1}$ and $L^{\prime \prime}$ being projective. Since $L^{\prime \prime}$ is projective, the second sequence splits, therefore, $L^{\prime} \cong P_{1} \oplus L^{\prime}$ is projective. Hence also the first sequence splits and we see that $L \cong P_{0} \oplus P_{1} \oplus L^{\prime \prime}$ is projective as well.

Proof. (of Theorem 12.1)

Let $A$ be a separable $\mathbf{C}(p)$-algebra, then the $\mathcal{R}$-module $\mathrm{EK}^{\mathbf{C}(p)}(A)$ is countably generated and exact. Let $\rho: \bigoplus_{\mathbb{N}} \mathcal{R} \rightarrow \mathrm{EK}^{\mathbf{C}(p)}(A)$ be an epimorphism of $\mathcal{R}$-modules and $L:=\operatorname{ker} \rho$. Then $L$ is an exact, countably generated $\mathcal{R}$-lattice, hence projective. Hence we have a projective resolution

$$
L \longmapsto \bigoplus_{\mathbb{N}} \mathcal{R} \rightarrow \operatorname{EK}^{\mathbf{C}(p)}(A)
$$

of length 1 . 



\section{A UCT for Actions of Finite Cyclic Groups}

\section{Introduction}

The aim of this chapter is to prove a Universal Coefficient Theorem for $C^{*}$-algebras equipped with an action of a finite cyclic group $G$. The main theorem reads as follows:

Theorem 13.1. Let $G$ be a finite cyclic group of order $\mathbf{o}$ and $A$ and $B$ separable $G-C^{*}$ algebras with $A$ in $\mathfrak{B}^{G}\left[\mathbf{o}^{-1}\right]$. Then there is a natural short exact sequence

$$
\operatorname{Ext}_{\mathfrak{A}}^{1}\left(\operatorname{LK}^{G}(S A), \operatorname{LK}^{G}(B)\right) \longmapsto \operatorname{KK}_{*}^{G}(A, B)\left[\mathbf{o}^{-1}\right] \rightarrow \operatorname{Hom}_{\mathfrak{A}}\left(\operatorname{LK}^{G}(A), \operatorname{LK}^{G}(B)\right) .
$$

Here, Hom and Ext are taken in the abelian category $\mathfrak{A}=\mathfrak{M o d}\left(\mathfrak{C}^{G}\left[\mathbf{o}^{-1}\right]\right)_{\mathrm{c}}^{\mathbb{Z} / 2}$.

In Section 14 we apply our general framework: We will first define the invariant $\mathrm{LK}^{G}$ by specifying a family $\mathcal{A}$ of objects in $\mathfrak{K}_{\mathfrak{K}}^{G}\left[\mathbf{o}^{-1}\right]$ for a general finite group $G$. We define the relevant bootstrap class in Section 14.2. The outcome is that in order to derive a $\mathrm{UCT}$, we have to show that modules over $\mathfrak{C}^{G}\left[\mathbf{o}^{-1}\right]$ have a projective resolution of length 1.

In Section 15, we develop tools to calculate $\mathfrak{C}^{G}$-the restriction of $\mathfrak{K}_{\mathfrak{K}}{ }^{G}$ to objects of the form $\mathcal{C}(G)^{H}, H \leq G$ for a finite abelian group $G$. In order to simplify calculations, we will first establish an involution on $\mathfrak{C}^{G}$ coming from Pontrijagin Duality (Section 15.1). We will then describe a set of generating morphisms of $\mathfrak{C}^{G}$ in Section 15.2 and their relations in Section 15.3.

The aim of Section 16 is to show that for a finite cyclic group $G$, modules over $\mathfrak{C}^{G}\left[\mathbf{o}^{-1}\right]$ indeed have a projective resolution of length 1 . We will start by introducing the concept of a split category over a family of Dedekind domains in Section 16.1. There, we will also show that modules over such categories have projective dimension 1 and that tensor products of split categories are again split. After having introduced these formal concepts, we focus on the case of $G$ being a cyclic group of prime power order and show that in this case $\mathfrak{C}^{G}\left[\mathbf{o}^{-1}\right]$ is split. Finally, in Section 16.3 , we show that for $G$ finite cyclic, $\mathfrak{C}^{G}\left[\mathbf{o}^{-1}\right]$ is the tensorproduct $\bigotimes_{i} \mathfrak{C}^{G_{i}}\left[\mathbf{p}_{\mathbf{i}}{ }^{-1}\right]$ with $G_{i}$ finite cyclic of prime power order and thereby split over a family of Dedekind domains. This will finish the proof. 


\section{The Framework}

\subsection{The Invariant}

Throughout this section let $G$ denote a finite group. We have already introduced $\mathfrak{K}_{\mathfrak{K}}{ }^{G}$ and stated that the suspension functor $S$ and triangles isomorphic to mapping cone triangles turn it into a triangulated category in Section 8.1.

Definition 14.1. Let $\mathcal{C}$ be a preadditive category and $R$ be a unital ring. Define $\mathfrak{C} \otimes R$ to be the category with the same objects as $\mathfrak{C}$ and morphisms given by

$$
\mathfrak{C} \otimes R(A, B):=\mathfrak{C}(A, B) \otimes_{\mathbb{Z}} R \text { for } A, B \in \in \mathfrak{C} .
$$

Composition is given by

$$
\mathfrak{C}(A, B) \otimes_{\mathbb{Z}} R \otimes_{\mathbb{Z}} \mathfrak{C}(B, C) \otimes_{\mathbb{Z}} R \cong \mathfrak{C}(A, B) \otimes_{\mathbb{Z}} \mathfrak{C}(B, C) \otimes_{\mathbb{Z}} R \otimes_{\mathbb{Z}} R \rightarrow \mathfrak{C}(A, C) \otimes_{\mathbb{Z}} R,
$$

where the last arrow is the tensor product of composition in $\mathfrak{C}$ and multiplication in $R$.

In general, $\mathfrak{T}$ being triangulated does not imply that $\mathfrak{T} \otimes R$ is triangulated. But in the special case $R=\mathbb{Z}\left[n^{-1}\right]$, this is true. For a preadditive category $\mathfrak{C}$, we will write $\mathfrak{C}\left[n^{-1}\right]$ for $\mathfrak{C} \otimes \mathbb{Z}\left[n^{-1}\right]$.

Theorem 14.2. Let $n \in \mathbb{Z} \backslash\{0\}$. Then $\mathfrak{K}_{\mathfrak{K}}^{G}\left[n^{-1}\right]$ is triangulated and

$$
\left(\_\right) \otimes \mathbb{Z}\left[n^{-1}\right]: \mathfrak{K} \mathfrak{K}^{G} \rightarrow \mathfrak{K} \mathfrak{K}^{G}\left[n^{-1}\right]
$$

is a triangulated functor.

Proof. This follows from Theorem 2.13. of [19].

Let $G$ be a finite abelian group of order $\mathbf{o}$. Set

$$
\mathcal{A}:=\left\{\mathcal{C}(G)^{H} \mid H \text { subgroup of } G\right\} .
$$

Note that $\mathcal{C}(G)^{H}$ is isomorphic to $\mathcal{C}(G / H)$ as a $G$ - $C^{*}$-algebra.

Definition 14.3. Let $\mathfrak{C}^{G}$ denote the full subcategory of $\mathfrak{K} \mathfrak{K}^{G}$ with objects $\mathcal{A}$ and $\mathfrak{C}^{G}\left[\mathbf{o}^{-1}\right]$ the full subcategory of $\mathfrak{K} \mathfrak{K}^{G}\left[\mathbf{o}^{-1}\right]$ with objects $\mathcal{A}$.

Definition 14.4. Define an invariant

$$
\mathrm{LK}^{G}: \mathfrak{K} \mathfrak{K}^{G}\left[\mathbf{o}^{-1}\right] \rightarrow \mathfrak{M o d}\left(\mathfrak{C}^{G}\left[\mathbf{o}^{-1}\right]\right)_{\mathrm{c}}^{\mathbb{Z} / 2}
$$

by $\mathrm{LK}^{G}:=F_{\mathcal{A}}$ (Definition 5.5).

Here, we write LK to abbreviate localized K-theory. Let $\mathfrak{R}^{G}\left[\mathbf{o}^{-1}\right]$ denote the category ring of $\mathfrak{C}^{G}\left[\mathbf{o}^{-1}\right]$. There is an isomorphism of abelian categories

$$
\mathfrak{M o d}\left(\mathfrak{C}^{G}\left[\mathbf{o}^{-1}\right]\right)_{\mathrm{c}}^{\mathbb{Z} / 2} \cong \mathfrak{M} \mathfrak{o d}\left(\mathfrak{R}^{G}\left[\mathbf{o}^{-1}\right]\right)_{\mathrm{c}}^{\mathbb{Z} / 2}, \quad M \mapsto \bigoplus_{A \in \mathcal{A}} M(A)
$$


Under this isomorphism, we obtain the following description of $\mathrm{LK}^{G}$ :

$$
\operatorname{LK}^{G}(A)=\bigoplus_{H \text { subgroup of } G} \operatorname{KK}_{*}^{G}(\mathcal{C}(G / H), A)\left[\mathbf{o}^{-1}\right] \cong \bigoplus_{H \text { subgroup of } G} \mathrm{~K}_{*}(H \ltimes A)\left[\mathbf{o}^{-1}\right]
$$

for a $G$-algebra $A$.

Here the last isomorphism comes from the fact that $\operatorname{Ind}_{H}^{G}$ is left adjoint to $\operatorname{Res}_{G}^{H}([27]$ Section 3.2) and the Green-Julg Theorem (Theorem 11.5). Hence on the level of abelian groups, the invariant is the direct sum of K-groups of crossed products with subgroups of $G$ tensored with $\mathbb{Z}\left[\mathbf{o}^{-1}\right]$.

All properties of the Hom-like invariants constructed for non-localized $G$-equivariant KK-theory also hold for $\mathrm{LK}^{G}$ (compare Section 8.2):

Lemma 14.5. If $B_{1} \longmapsto B_{2} \rightarrow B_{3}$ is a $G$-equivariant extension, then there is a natural 6-term exact sequence

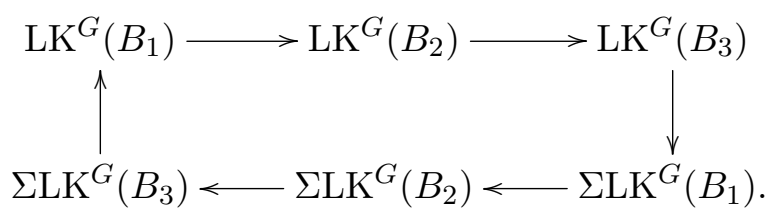

Proof. Follows from Lemma 8.2 since $\mathbb{Z}\left[\mathbf{o}^{-1}\right]$ is flat ([47], 3.2.2).

Proposition 14.6 (Thom isomorphism). Let $B$ be a separable $G \times \mathbb{R}$-algebra, then there is an isomorphism

$$
\Sigma \mathrm{LK}^{G}(B) \cong \mathrm{LK}^{G}(\mathbb{R} \ltimes B) .
$$

Proof. Follows from Proposition 8.3.

Proposition 14.7 (Pimsner-Voiculescu exact sequence). Let $B$ be a separable $G \times \mathbb{Z}$ algebra, then there is a natural 6 -term exact sequence

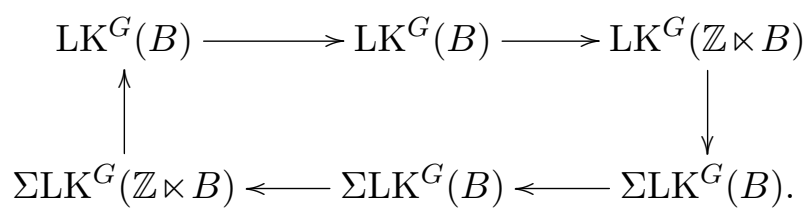

Proof. Follows from Proposition 8.4 since $\mathbb{Z}\left[\mathbf{o}^{-1}\right]$ is flat.

\subsection{The Bootstrap Class}

Definition 14.8. Define the botstrap class $\mathfrak{B}^{G}$ as the localizing subcategory of $\mathfrak{K} \mathfrak{K}^{G}$ which is generated by $\mathcal{A}$. Let $\mathfrak{B}^{G}\left[\mathbf{o}^{-1}\right]$ be the localizing subcategory of $\mathfrak{K}_{\mathfrak{K}}^{G}\left[\mathbf{o}^{-1}\right]$ which is generated by $\mathcal{A}$.

Note that $\mathfrak{B}^{G}$ is contained in $\mathfrak{B}^{G}\left[\mathbf{o}^{-1}\right]$. By Theorem 9.5 , every commutative $G$-algebra is in $\mathfrak{B}^{G}$. 


\section{A UCT for Actions of Finite Cyclic Groups}

Remark 14.9. By our general framework (Theorem 7.1), in order to prove Theorem 13.1, we have to show that $\operatorname{LK}^{G}(A)$ has projective dimension 1 in $\mathfrak{M o d}\left(\mathfrak{C}^{G}\left[\mathbf{o}^{-1}\right]\right)_{\mathrm{c}}^{\mathbb{Z} / 2}$ for all $A \in \in \mathfrak{B}^{G}\left[\mathbf{o}^{-1}\right]$. In fact, we will show that for a finite cyclic group $G$, all objects in $\mathfrak{M o d}\left(\mathfrak{C}^{G}\left[\mathbf{o}^{-1}\right]\right)_{\mathrm{C}}^{\mathbb{Z} / 2}$ have a projective resolution of length 1 .

\section{Basic Calculations in $\mathfrak{C}^{G}$}

\subsection{Duality}

In the following, let $G$ denote a finite abelian group. $H$ and $K$ will always denote subgroups of $G$.

Recall that $\mathbf{C}(k)=\mathbb{Z} / k \mathbb{Z}$ denotes the cyclic group of order $k$. Define

$$
\chi_{k}: \mathbf{C}(k) \times \mathbf{C}(k) \rightarrow S^{1}, \quad([m],[n]) \mapsto \exp \left(2 \pi \mathrm{i} \frac{m n}{k}\right)
$$

By a well-known classification result for finitely generated abelian groups ([16] Theorem 10.3), there is an isomorphism

$$
\Phi: G \cong \bigoplus_{j=1}^{n} \mathbf{C}\left(k_{j}\right)
$$

for $j=1, \ldots, n$ and $k_{j} \in \mathbb{N}$. Let $\phi_{j}$ denote the projection onto the $j$ th component. Define

$$
\chi: G \times G \rightarrow S^{1}, \quad \chi(g, h)=\prod_{j} \chi_{k_{j}}\left(\phi_{j}(g), \phi_{j}(h)\right) .
$$

$\chi$ is a group homomorphism, which depends on the choice of the isomorphism $\Phi$. Let us fix $\Phi$ and thereby $\chi$ once and for all. We have $\chi(g, h)=\chi(h, g)$.

Definition 15.1. Set

$$
H^{\perp}:=\{g \in G \mid \chi(g, h)=1 \forall h \in H\} .
$$

$H^{\perp}$ is a subgroup of $G$.

\section{Proposition 15.2.}

$$
G / H^{\perp} \rightarrow \widehat{H}, \quad[g] \mapsto(h \mapsto \chi(g, h)),
$$

is an isomorphism.

Proof. For $[g] \in G / H^{\perp}$, let $\chi_{[g]} \in \widehat{H}$ denote the character $h \mapsto \chi(g, h)$. Let us first check that $g \mapsto \chi_{g}$ gives an isomorphism $G \cong \widehat{G}$. Recall that there is an isomorphism $\Phi: G \cong \bigoplus_{j=1}^{n} \mathbf{C}\left(k_{j}\right)$. Since the sum is finite, we have $\widehat{G} \cong \bigoplus_{j=1}^{n} \widehat{\mathbf{C}\left(k_{j}\right)}$. By definition of $\chi$, the diagram

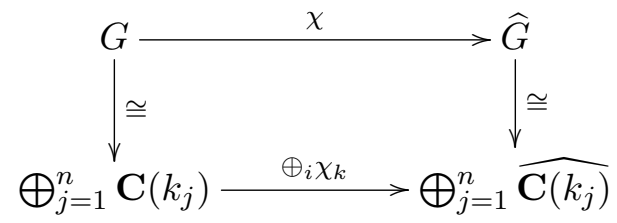


commutes, hence it suffices to show that

$$
\left.\mathbf{C}(k) \rightarrow \widehat{\mathbf{C}(k)}, \quad[n] \mapsto\left([m] \mapsto \exp \left(2 \pi \mathrm{i} \frac{m n}{k}\right)\right)\right)
$$

is an isomorphism. But this is elementary.

The short exact sequence $H \longmapsto G \rightarrow G / H$ induces a short exact sequence $\widehat{G / H} \longmapsto$ $\widehat{G} \rightarrow \widehat{H}$ since $G$ is finite ([39] Proposition 2). This already shows that

$$
G \stackrel{\chi}{\rightarrow} \widehat{G} \rightarrow \widehat{H}
$$

is surjective. By definition, $H^{\perp}$ is the kernel of this map, this shows the claim.

Lemma 15.3. $\left(H^{\perp}\right)^{\perp}=H$.

Proof. Let $h \in H$ and $g \in H^{\perp}$, then $\chi(h, g)=\chi(g, h)=1$, hence $H \subseteq\left(H^{\perp}\right)^{\perp}$. Since $H \cong \widehat{H} \cong G / H^{\perp}$, we have $|G|=|H| \cdot\left|H^{\perp}\right|$. Therefore, $|H|=\left|\left(H^{\perp}\right)^{\perp}\right|$, this shows that $H=\left(H^{\perp}\right)^{\perp}$.

If $H$ is a subgroup of $G$, then the normalization of the Haar measure on $H$ is chosen such that points have mass 1 . Let $(A, \alpha)$ and $(B, \beta)$ be $G$-algebras. Note that the crossed product $G \ltimes{ }_{\alpha} A$ as a vector space is given as $\mathcal{C}(G, A)$. We will omit the index $\alpha$ if there is no danger of confusion. Since $G$ is abelian, there is a dual action $\widehat{\alpha}$ of $\widehat{G}$ on $G \ltimes A$, which we will interpret as an action of $G$ via the isomorphism $\widehat{G} \cong G$. $\widehat{\alpha}$ is then given by $\widehat{\alpha}_{l}(f)(k):=\chi(l, k) f(k)$. By the universal property of $\mathrm{KK}^{G}([26]$, Theorem 50) we obtain an endofunctor

$$
G \ltimes\left(\_\right): \mathfrak{K K}^{G} \rightarrow \mathfrak{K}^{G} .
$$

In [1], S. Baaj and G. Skandalis examine $G \ltimes\left(\_\right)$for a general Hausdorff topological group $G$. We can derive the following explicit description of $G \ltimes\left(\_\right)$for an abelian $G$ from [1] 6.6 and 6.17: Let $x=[(\mathcal{E}, T, \gamma)] \in \mathrm{KK}^{G}(A, B)$. Then $G \ltimes x$ is represented by $(G \ltimes \mathcal{E}, \widehat{T}, \widehat{\gamma})$, where $G \ltimes \mathcal{E}=\mathcal{C}(G, E)$ as a vector space and the expressions for inner product, left and right multiplication specialize to:

$$
\begin{gathered}
(a \xi)(k)=\int_{G} a(l) \gamma_{l}(\xi(k-l)) \mathrm{d} l,(\xi b)(k)=\int_{G} \xi(l) \beta_{l}(b(k-l)) \mathrm{d} l \\
\langle\xi, \eta\rangle(k)=\int_{G} \beta_{-l}(\langle\xi(l), \eta(k+l)\rangle) \mathrm{d} l
\end{gathered}
$$

for $\xi, \eta \in G \ltimes \mathcal{E}, b \in G \ltimes B$ and $a \in G \ltimes A$. The operator $\widehat{T}$ is given by $(\widehat{T}(\xi))(k)=T(\xi(k))$ and the action $\hat{\gamma}$ by $\widehat{\gamma}_{l}(\xi)(k)=\chi(l, k) \xi(k)$.

Applying the crossed product twice yields a $G$-algebra $\left(G \ltimes \widehat{\alpha}_{\alpha} G \ltimes{ }_{\alpha} A\right.$, $\left.\widehat{\widehat{\alpha}}\right)$, which is naturally $K K^{G}$-equivalent to $A$ by the Takai Duality Theorem [43]. Thus $G \ltimes\left(\_\right)$is an autofunctor of $\mathfrak{K}_{\mathfrak{K}}{ }^{G}$, which is involutive up to a natural isomorphism, the natural transformation $T$. This observation will be used to simplify the computation of $\mathfrak{C}^{G}$. It will be useful to explicitly describe $T$. 


\section{A UCT for Actions of Finite Cyclic Groups}

Let $\left(\mathbb{K}\left(L^{2}(G, A)\right), \operatorname{Ad}_{\rho \otimes \alpha}\right)$ be the compact operators on the Hilbert $A$-module $L^{2}(G, A)$ equipped with the adjoint action, where $G$ acts on $L^{2}(G, A)$ via

$$
(\rho \otimes \alpha)_{l}(f)(k)=\alpha_{l}(f(k+l)), \quad f \in L^{2}(G, A) .
$$

Lemma 15.6. There is an equivariant *-isomorphism

$$
\Phi_{A}:\left(G \ltimes_{\widehat{\alpha}} G \ltimes_{\alpha} A, \widehat{\hat{\alpha}}\right) \rightarrow\left(\mathbb{K}\left(L^{2}(G, A)\right), \operatorname{Ad}_{\rho \otimes \alpha}\right),
$$

which is given by

$$
\Phi_{A}(F)(f)(r)=\int_{G} \int_{G} \alpha_{r}^{-1}(F(t, s)) \chi(t, r-s) f(r-s) \mathrm{d} t \mathrm{~d} s
$$

for $F \in G \ltimes_{\widehat{\alpha}} G \ltimes_{\alpha} A={ }_{v s} \mathcal{C}(G \times G, A)$ and $f \in L^{2}(G, A)$.

Proof. This is a standard result, see for example [48], Theorem 7.1.

Let us denote by $E_{A} \in \mathrm{KK}^{G}\left(\mathbb{K}\left(L^{2}(G, A)\right), A\right)$ the KK-element, which is given by the Morita-Rieffel imprimitivity bimodule $\left(L^{2}(G, A), \rho \otimes \alpha\right)$. Then the natural transformation

$$
T: G \ltimes G \ltimes\left(\_\right) \Rightarrow \text { id }
$$

is given by the $\mathrm{KK}^{G}$-equivalence $T_{A}=\left[\Phi_{A}\right] \cdot E_{A}$. That $T$ is indeed a natural transformation, i.e., that for $G$-algebras $A$ and $B$ and $x \in \mathrm{KK}^{G}(A, B)$, we have

$$
G \ltimes G \ltimes x \cdot T_{B}=T_{A} \cdot x,
$$

follows from [1], Théorème 6.20 .

For calculations, it will be useful to turn $G \ltimes\left(\_\right)$into an actual involution on $\mathfrak{C}^{G}$.

We are especially interested in the crossed product $G \ltimes \mathcal{C}(G)^{H}$, which, as a vector space, will be identified with $\mathcal{C}(G \times G)\{0\} \times H$.

There is an embedding

$$
\iota: C^{*} H \hookrightarrow C^{*} G, \quad \iota(f)(g):=\left\{\begin{array}{ll}
f(g) & \text { if } g \in H, \\
0 & \text { otherwise }
\end{array} .\right.
$$

We will regard $C^{*} H$ as a subalgebra of $C^{*} G$ via this embedding.

Fourier transformation gives an equivariant isomorphism

$$
\mathcal{F}:(\mathcal{C}(G), \lambda) \rightarrow\left(C^{*} G, \widehat{\tau}\right), \quad \mathcal{F}(f)(g)=\frac{1}{|G|} \int_{G} \chi\left(g, g^{\prime}\right) f\left(g^{\prime}\right) \mathrm{d} g^{\prime}
$$

Lemma 15.7. $\mathcal{F}\left(\mathcal{C}(G)^{H}\right)=C^{*} H^{\perp}$.

Proof. Let $g \in G \backslash H^{\perp}, f \in \mathcal{C}(G)^{H}$ and $\left(g_{i}\right)_{i \in I}$ a set of representatives of $G / H$. Then $\chi_{[g]} \in \widehat{H}$ is non-trivial in $\widehat{H}$, hence $\sum_{h \in H} \chi_{[g]}(h)=0$, hence

$$
\mathcal{F}(f)(g)=\frac{1}{|G|} \sum_{i} \sum_{h \in H} \chi\left(g, g_{i}+h\right) f\left(g_{i}\right)=\frac{1}{|G|} \sum_{i} \chi\left(g, g_{i}\right) f\left(g_{i}\right)\left(\sum_{h \in H} \chi_{[g]}(h)\right)=0 .
$$

This shows $\mathcal{F}\left(\mathcal{C}(G)^{H}\right) \subseteq C^{*} H^{\perp}$. Counting dimensions shows equality. 
Let us denote the restriction $\mathcal{F}_{\mid \mathcal{C}(G)^{H}}: \mathcal{C}(G)^{H} \cong C^{*} H^{\perp}$ by $\mathcal{F}_{H}$.

Recall that $G \ltimes \mathcal{C}(G)^{H}=G \ltimes \operatorname{Ind}_{H}^{G} \mathbb{C}$ is Morita-Rieffel equivalent to $C^{*} H$ ([48], Theorem 4.22). Composing the corresponding imprimitivity bimodule with $\mathcal{F}_{H^{\perp}}^{-1}$ on the right, we obtain an imprimitivity bimodule $X^{H}$, which gives a Morita-Rieffel equivalence between $G \ltimes \mathcal{C}(G)^{H}$ and $\mathcal{C}(G)^{H^{\perp}}$.

Definition 15.8. Define the involution $\mathcal{I}: \mathfrak{C}^{G} \rightarrow \mathfrak{C}^{G}$ by

$$
\mathcal{I}\left(\mathcal{C}(G)^{H}\right)=\mathcal{C}(G)^{H^{\perp}}, \quad \mathcal{I}(x)=\left[X^{H}\right]^{-1} \cdot(G \ltimes x) \cdot\left[X^{K}\right]
$$

for $x \in \mathrm{KK}^{G}\left(\mathcal{C}(G)^{H}, \mathcal{C}(G)^{K}\right)$.

$\mathcal{I}$ is obviously a functor. A computation shows that $G \ltimes\left[X^{H}\right] \cdot\left[X^{H^{\perp}}\right]=T_{\mathcal{C}(G)^{H}}$ (Proposition 23.1 in the appendix). Together with $(G \ltimes G \ltimes x) \cdot T_{B}=T_{A} \cdot x$ for $x \in$ $\mathrm{KK}^{G}(A, B)$, it is easy to check that $\mathcal{I}$ is an involution: Let $x \in \mathrm{KK}^{G}\left(\mathcal{C}(G)^{H}, \mathcal{C}(G)^{K}\right)$, then

$$
\begin{aligned}
\mathcal{I}^{2}(x) & =\left[X^{H^{\perp}}\right]^{-1} \cdot G \ltimes\left[X^{H}\right]^{-1} \cdot(G \ltimes G \ltimes x) \cdot G \ltimes\left[X^{K}\right] \cdot X^{K^{\perp}} \\
& =T_{\mathcal{C}(G)^{H}}^{-1} \cdot(G \ltimes G \ltimes x) \cdot T_{\mathcal{C}(G)^{K}} \\
& =T_{\mathcal{C}(G)^{K}}^{-1} \cdot T_{\mathcal{C}(G)^{K}} \cdot x=x .
\end{aligned}
$$

\subsection{Generators of $\mathfrak{C}^{G}$}

In this subsection we will introduce some morphisms in $\mathfrak{C}^{G}$ and then show that they serve as basic building blocks of generators of $\mathfrak{C}^{G}$.

Definition 15.9. For $g \in G$, let us define elements in $\mathrm{KK}^{G}\left(\mathcal{C}(G)^{H}, \mathcal{C}(G)^{H}\right)$ by

$$
t_{g}^{H}:=\left[\left(\lambda_{g}\right)_{\mid \mathcal{C}(G)^{H}}\right] \text { and } r_{g}^{H}:=\left[\chi_{g}\right] \otimes_{\mathbb{C}} 1_{\mathcal{C}(G)^{H}}
$$

In words, $t_{g}^{H}$ is given by translation by the group element $g$ and $r_{g}^{H}$ by the outer tensor product with the character $\chi_{g}$.

Lemma 15.10. Let $g \in G$ and $K, H$ be subgroups of $G$ and $x \in \mathrm{KK}_{*}^{G}\left(\mathcal{C}(G)^{K}, \mathcal{C}(G)^{H}\right)$, then $t_{g}^{H} \cdot x=x \cdot t_{g}^{H}$ and $r_{g}^{K} \cdot x=x \cdot r_{g}^{H}$.

Proof. The first equality follows since $\left[\alpha_{g}\right] \cdot x=x \cdot\left[\beta_{g}\right]$ for $G$-algebras $(A, \alpha),(B, \beta)$, $g \in G$ and $x \in \mathrm{KK}^{G}(A, B)$ and the second since the outer tensor product with elements in $K K^{G}(\mathbb{C}, \mathbb{C})$ is commutative.

Definition 15.11. Let $K \leq H \leq G$, let $\iota_{H}^{K} \in \mathrm{KK}^{G}\left(\mathcal{C}(G)^{H}, \mathcal{C}(G)^{K}\right)$ be the class of the inclusion $\mathcal{C}(G)^{H} \hookrightarrow \mathcal{C}(G)^{K}$.

Still assuming $K \leq H \leq G$, the right module structure over $\mathcal{C}(G)^{H}$ and an inner product given by

$$
\langle x, y\rangle(s)=\int_{H} \overline{x(s+t)} y(s+t) d t, x, y \in \mathcal{C}(G)^{K}
$$

turn $\mathcal{C}(G)^{K}$ into a Kasparov- $\mathcal{C}(G)^{K}-\mathcal{C}(G)^{H}$-module, which will be denoted by $R_{K}^{H}$. 


\section{A UCT for Actions of Finite Cyclic Groups}

Definition 15.13. Let $\rho_{K}^{H}=\left[\left(R_{K}^{H}, 0, \lambda\right)\right] \in \mathrm{KK}^{G}\left(\mathcal{C}(G)^{K}, \mathcal{C}(G)^{H}\right)$ denote the corresponding KK-element.

Recall that for $H \leq G$, there are induction and restriction functors $\operatorname{Ind}_{H}^{G}: \mathfrak{K} \mathfrak{K}^{H} \rightarrow \mathfrak{K} \mathfrak{K}^{G}$ and $\operatorname{Res}_{G}^{H}: \mathfrak{K K}^{G} \rightarrow \bar{K}_{\mathfrak{K}}{ }^{H}$ (see [23] Definition 3.1 and Section 3.6 or [27], Section 3.6 for a more functorial approach). $\operatorname{Res}_{G}^{H}$ is given by restricting the action to the subgroup $H$. If $(B, \beta)$ is an $H$-algebra, then in our case (of $G$ being finite abelian), $\operatorname{Ind}_{H}^{G}(B, \beta)$ is given as a $C^{*}$-subalgebra of $\mathcal{C}(G, B)$ :

$$
\operatorname{Ind}_{H}^{G}(B, \beta)=\left\{f \in \mathcal{C}(G, B) \mid f(g+h)=\beta_{-h}(f(g))\right\},
$$

where $G$ acts by left translation. There is an isomorphism of functors $\operatorname{Ind}_{H}^{G} \operatorname{Res}_{G}^{H}=$ $\left(\_\right) \otimes_{\mathbb{C}} \mathcal{C}(G)^{H}([27]$, Equation (18)). Furthermore we will use the adjointness relations

$$
\mathrm{KK}^{G}\left(A, \operatorname{Ind}_{H}^{G} B\right) \cong \mathrm{KK}^{H}\left(\operatorname{Res}_{G}^{H} A, B\right) \text { and } \mathrm{KK}^{G}\left(\operatorname{Ind}_{H}^{G} B, A\right) \cong \mathrm{KK}^{H}\left(B, \operatorname{Res}_{G}^{H} A\right)
$$

for a $G$-algebra $A$ and an $H$-algebra $B$ ([27], Equations (19) and (20)).

Definition 15.14. Let $H$ and $L$ be arbitrary subgroups of $G$. Set

$$
\mu_{H}^{L}:=\rho_{H}^{H+L} \cdot \iota_{H+L}^{L} .
$$

Proposition 15.15. Let $L$ and $H$ be arbitrary subgroups of $G$. Let $\left(g_{i}\right)_{i \in I}$ be a set of representatives of $G /(H+L)$ and $\left(s_{j}\right)_{j \in J}$ a set of representatives of $G /(H \cap L)^{\perp}$. Then $\mathrm{KK}_{*}^{G}\left(\mathcal{C}(G)^{H}, \mathcal{C}(G)^{L}\right)$ is a free $\mathbb{Z}$-module, concentrated in degree 0 with basis

$$
\left(t_{g_{i}}^{H} \cdot \mu_{H}^{L} \cdot r_{s_{j}}^{L}\right)_{(i, j) \in I \times J} .
$$

Proof. We have

$$
\rho_{H}^{H+L} \cdot t_{g_{i}}^{L+H} \cdot \iota_{H+L}^{L} \cdot r_{s_{j}}^{L}=t_{g_{i}}^{H} \cdot \rho_{H}^{H+L} \cdot \iota_{H+L}^{L} \cdot r_{s_{j}}^{L}=t_{g_{i}}^{H} \cdot \mu_{H}^{L} \cdot r_{s_{j}}^{L}
$$

by Lemma 15.10. Hence it is sufficient to show that $\operatorname{KK}_{*}^{G}\left(\mathcal{C}(G)^{H}, \mathcal{C}(G)^{L}\right)$ is a free $\mathbb{Z}$-module, concentrated in degree 0 with basis

$$
\left(\rho_{H}^{H+L} \cdot t_{g_{i}}^{L+H} \cdot \iota_{H+L}^{L} \cdot r_{s_{j}}^{L}\right)_{(i, j) \in I \times J} .
$$

The unital embedding $u: \mathbb{C} \hookrightarrow \mathcal{C}(H)^{H \cap L}=\operatorname{Ind}_{H \cap L}^{H} \operatorname{Res}_{H}^{H \cap L} \mathbb{C}$ is the unit of the adjointness relation

$$
\mathrm{KK}^{H \cap L}\left(\mathbb{C}, \operatorname{Res}_{H}^{H \cap L}\left(\_\right)\right) \cong \mathrm{KK}^{H}\left(\operatorname{Ind}_{H \cap L}^{H} \mathbb{C}, \ldots\right) .
$$

The counit of the adjointness relation

$$
\mathrm{KK}^{G}\left(\operatorname{Ind}_{H}^{G}\left(\_\right), \mathcal{C}(G)^{L}\right) \cong \mathrm{KK}^{H}\left(\ldots, \operatorname{Res}_{G}^{H} \mathcal{C}(G)^{L}\right)
$$

will be denoted by $c$ : $\operatorname{Ind}_{H}^{G} \operatorname{Res}_{G}^{H} \mathcal{C}(G)^{L} \rightarrow \mathcal{C}(G)^{L}$. $c$ is given by $[\tau] \cdot\left(\rho_{H}^{G} \otimes_{\mathbb{C}} 1_{\mathcal{C}(G)^{L}}\right)$, where

$$
\tau: \operatorname{Ind}_{H}^{G} \operatorname{Res}_{G}^{H} \mathcal{C}(G)^{L} \cong \mathcal{C}(G)^{H} \otimes_{\mathbb{C}} \mathcal{C}(G)^{L}, \tau(f)\left(g, g^{\prime}\right)=f(g)\left(g^{\prime}-g\right) .
$$


Note that in $\mathfrak{K} \mathfrak{K}^{H}$, there is an isomorphism

$$
\mathcal{C}(G)^{L} \cong \bigoplus_{I} \mathcal{C}\left(-g_{i}+H+L\right)^{L} \cong \bigoplus_{I} \mathcal{C}(H)^{H \cap L}
$$

Its inverse is given by $\bigoplus_{I} j_{g_{i}}$, where

$$
j_{g_{i}}: \mathcal{C}(H)^{H \cap L} \hookrightarrow \mathcal{C}(G)^{L}, \quad j_{g_{i}}(f)(g)= \begin{cases}f(h) & \text { if } g=-g_{i}+h+l, h \in H, l \in L, \\ 0 & \text { otherwise }\end{cases}
$$

Following the chain of isomorphisms

$$
\begin{aligned}
\bigoplus_{I} \mathrm{KK}^{H \cap L}(\mathbb{C}, \mathbb{C}) & \stackrel{u^{*} \circ \operatorname{Ind}_{H \cap L}^{H}}{\longrightarrow} \bigoplus_{I} \mathrm{KK}^{H}\left(\mathbb{C}, \mathcal{C}(H)^{H \cap L}\right) \\
& \stackrel{\bigoplus_{I}\left(j_{g_{i}}\right)_{*}}{\longrightarrow} \mathrm{KK}^{H}\left(\mathbb{C}, \mathcal{C}(G)^{L}\right) \\
& \stackrel{c_{*} \circ \operatorname{Ind}_{H}^{G}}{\longrightarrow} \mathrm{KK}^{G}\left(\mathcal{C}(G)^{H}, \mathcal{C}(G)^{L}\right)
\end{aligned}
$$

we see that $\mathrm{KK}^{G}\left(\mathcal{C}(G)^{H}, \mathcal{C}(G)^{L}\right)$ is a free $\mathbb{Z}$-module, concentrated in degree 0 with basis

$$
\left(\operatorname{Ind}_{H}^{G}\left(u \cdot \operatorname{Ind}_{H \cap L}^{H}([\phi]) \cdot\left[j_{g_{i}}\right]\right) \cdot c\right)_{i \in I, \phi \in \widehat{H \cap L}} .
$$

Note that $J \rightarrow \widehat{H \cap L}, j \mapsto \operatorname{Res}_{G}^{H \cap L}\left(\chi_{s_{j}}\right)$ is a bijection. Since

$\operatorname{Ind}_{H}^{G}\left(\operatorname{Ind}_{H \cap L}^{H} \operatorname{Res}_{G}^{H \cap L}\left(\left[\chi_{s_{j}}\right]\right) \cdot\left[j_{g_{i}}\right]\right) \cdot c=\left(\left[\chi_{s_{j}}\right] \otimes_{\mathbb{C}} 1_{\operatorname{Ind}_{H}^{G}} \mathcal{C}(H)^{H \cap L}\right) \cdot \operatorname{Ind}_{H}^{G}\left[j_{g_{i}}\right] \cdot c=\operatorname{Ind}_{H}^{G}\left[j_{g_{i}}\right] \cdot c \cdot r_{s_{j}}^{L}$,

it suffices to show that

$$
\operatorname{Ind}_{H}^{G} u \cdot \operatorname{Ind}_{H}^{G}\left[j_{g_{i}}\right] \cdot c=\rho_{H}^{H+L} \cdot m_{g_{i}}^{L+H} \cdot \iota_{H+L}^{L} \in \mathrm{KK}^{G}\left(\mathcal{C}(G)^{H}, \mathcal{C}(G)^{L}\right) .
$$

Calculating the composition

$$
T_{i}:=\mathcal{C}(G)^{H} \stackrel{\operatorname{Ind}_{H}^{G} u}{\longrightarrow} \operatorname{Ind}_{H}^{G} \mathcal{C}(H)^{H \cap L} \stackrel{\operatorname{Ind}_{H}^{G} j_{g_{i}}}{\longrightarrow} \operatorname{Ind}_{H}^{G} \mathcal{C}(G)^{L} \stackrel{\tau}{\rightarrow} \mathcal{C}(G)^{H} \otimes_{\mathbb{C}} \mathcal{C}(G)^{L}
$$

yields

$$
T_{i}: \mathcal{C}(G)^{H} \rightarrow \mathcal{C}(G)^{H} \otimes_{\mathbb{C}} \mathcal{C}(G)^{L}, \quad T(f)\left(g, g^{\prime}\right)=f(g) 1_{H+L-g_{i}}\left(g-g^{\prime}\right),
$$

where $1_{H+L-g_{i}}$ denotes the characteristic function of the set $H+L-g_{i}$. Hence we have to show that there is an isomorphism of Kasparov $\mathcal{C}(G)^{H}-\mathcal{C}(G)^{L}$-modules

$$
\left(T_{i}\right)^{*}\left(R_{H}^{G} \otimes_{\mathbb{C}} 1_{\mathcal{C}(G)^{L}}\right) \cong\left(\iota_{H+L}^{L} \circ \lambda_{g_{i}}\right)_{*} R_{H}^{H+L} .
$$

In the following, we will identify $\mathcal{C}(G)^{H} \otimes_{\mathbb{C}} \mathcal{C}(G)^{L}$ with $\mathcal{C}(G \times G)^{H \times L}$. Since $\left.\left(T_{i}\right)^{*}\left(R_{H}^{G} \otimes_{\mathbb{C}} 1_{\mathcal{C}(G)^{L}}\right)\right)==_{\text {vs }}$ $T_{i} \cdot \mathcal{C}(G)^{H} \otimes_{\mathbb{C}} \mathcal{C}(G)^{L}$, the left-hand-side is given as a vector space by

$$
T_{i}(1) \cdot \mathcal{C}(G)^{H} \otimes \mathcal{C}(G)^{L}=_{\text {vs }}\left\{f \in \mathcal{C}(G \times G)^{H \times L} \mid f\left(g, g^{\prime}\right)=1_{H+L-g_{i}}\left(g-g^{\prime}\right) f\left(g, g^{\prime}\right)\right\} .
$$




\section{A UCT for Actions of Finite Cyclic Groups}

The left module structure is given by $T_{i}$, the right module structure by $1 \otimes \mathrm{id}_{\mathcal{C}(G)^{L}}$ and the inner product by $\left\langle f, f^{\prime}\right\rangle\left(g^{\prime}\right)=\int_{G} \overline{f\left(g, g^{\prime}\right)} f^{\prime}\left(g, g^{\prime}\right) \mathrm{d} g$.

To describe $\left(\iota_{H+L}^{L} \circ \lambda_{g_{i}}\right)_{*} R_{H}^{H+L}$, let us first set

$$
\Lambda_{i}:=\iota_{H+L}^{L} \circ \lambda_{g_{i}}: \mathcal{C}(G)^{H+L} \rightarrow \mathcal{C}(G)^{L}, \Lambda_{i}(f)(g)=f\left(g-g_{i}\right) .
$$

Then $\left(\iota_{H+L}^{L} \circ \lambda_{g_{i}}\right)_{*} R_{H}^{H+L}=\mathcal{C}(G)^{H} \otimes_{\Lambda_{i}} \mathcal{C}(G)^{L}$. Left and right module structure are given by $\operatorname{id}_{\mathcal{C}(G)^{H}} \otimes_{\Lambda_{i}} 1$ and $1 \otimes_{\Lambda_{i}} \operatorname{id}_{\mathcal{C}(G)^{L}}$, respectively, and the inner product is given by

$$
\left\langle f_{0} \otimes_{\Lambda_{i}} f_{0}^{\prime}, f_{1} \otimes_{\Lambda_{i}} f_{1}^{\prime}\right\rangle(g)=\overline{f_{0}^{\prime}(g)} \int_{H+L} \overline{f_{0}\left(g-g_{i}+r\right)} f_{1}\left(g-g_{i}+r\right) d r f_{1}^{\prime}(g) .
$$

Hence $\left(\iota_{H+L}^{L} \circ \lambda_{g_{i}}\right)_{*} R_{H}^{H+L}$ as a vector space is a quotient and $\left(T_{i}\right)^{*}\left(R_{H}^{G} \otimes_{\mathbb{C}} 1_{\mathcal{C}(G)^{L}}\right)$ is a subspace of $\mathcal{C}(G \times G)^{H \times L}$. Using the description of the inner products above, a straightforward calculation show that

$$
\phi: \mathcal{C}(G \times G)^{H \times L} \rightarrow \mathcal{C}(G \times G)^{H \times L}, \quad f \mapsto\left(\left(g, g^{\prime}\right) \mapsto 1_{H+L-g_{i}}\left(g-g^{\prime}\right) f\left(g, g^{\prime}\right)\right)
$$

factors through an injective map $\Phi: \mathcal{C}(G)^{H} \otimes_{\Lambda_{i}} \mathcal{C}(G)^{L} \rightarrow \mathcal{C}(G)^{H} \otimes_{\mathbb{C}} \mathcal{C}(G)^{L}$, which intertwines the inner products. It is also easy to check that $\operatorname{im} \Phi=T_{i}(1) \cdot \mathcal{C}(G)^{H} \otimes \mathcal{C}(G)^{L}$ (i.e., that $\Phi$ is surjective) and that it intertwines left and right multiplication. Therefore, $\Phi$ is an isomorphism of Kasparov $\mathcal{C}(G)^{H}-\mathcal{C}(G)^{L}$-modules

$$
\left(\iota_{H+L}^{L} \circ \lambda_{g_{i}}\right)_{*} R_{H}^{H+L} \stackrel{\cong}{\longrightarrow}\left(T_{i}\right)^{*}\left(R_{H}^{G} \otimes_{\mathbb{C}} 1_{\mathcal{C}(G)^{L}}\right) .
$$

\subsection{Relations in $\mathfrak{C}^{G}$}

In this subsection we will derive some properties of the basic building blocks for generators of $\mathfrak{C}^{G}$.

Lemma 15.16. One has $I\left(r_{g}^{H}\right)=t_{g}^{H^{\perp}}$ and $I\left(t_{g}^{H}\right)=r_{g}^{H^{\perp}}$.

Proof. By definition, $I\left(r_{g}^{H}\right)=\left[X^{H}\right]^{-1} \cdot\left(G \ltimes\left(\left[\chi_{g}\right] \otimes_{\mathbb{C}} 1_{\mathcal{C}(G)^{H}}\right)\right) \cdot\left[X^{H}\right]$. Let $\lambda^{H}$ denote the translation action restricted to $\mathcal{C}(G)^{H}$. Using the formulas on Baaj-Skandalis duality (formula (15.4) and (15.5)), it is easy to check that

$$
G \ltimes\left(\left[\chi_{g}\right] \otimes_{\mathbb{C}} 1_{\mathcal{C}(G)^{H}}\right)=\left[\left(\widehat{\lambda^{H}}\right)_{g}\right] \in \mathrm{KK}^{G}\left(G \ltimes \mathcal{C}(G)^{H}, G \ltimes \mathcal{C}(G)^{H}\right),
$$

where $\widehat{\lambda^{H}}$ is the dual action of $G$ on $G \ltimes \mathcal{C}(G)^{H}$. Since $\left[\alpha_{g}\right] \cdot x=x \cdot\left[\beta_{g}\right]$ for $G$-algebras $(A, \alpha),(B, \beta), g \in G$ and $x \in \mathrm{KK}^{G}(A, B)$, we obtain the first equality. Applying $\mathcal{I}$ yields the second equality.

Lemma 15.17. Let $h \in H$, then $t_{h}^{H}=1_{\mathcal{C}(G)^{H}}$ and $r_{h}^{H^{\perp}}=1_{\mathcal{C}(G)^{H^{\perp}}}$.

Proof. $t_{h}^{H}=1_{\mathcal{C}(G)^{H}}$ for $h \in H$ is trivial, the other equality follows by applying $\mathcal{I}$. 
Lemma 15.18. One has $\mathcal{I}\left(\iota_{H}^{K}\right)=\rho_{H^{\perp}}^{K^{\perp}}$ and $\mathcal{I}\left(\rho_{K}^{H}\right)=\iota_{K^{\perp}}^{H^{\perp}}$.

Proof. By definition, $\mathcal{I}\left(\iota_{K}^{H}\right)=\left(X^{H}\right)^{-1} \cdot\left(G \ltimes \iota_{H}^{K}\right) \cdot\left(X^{K}\right)$. Hence we have to show that the Kasparov $G \ltimes \mathcal{C}(G)^{H}-\mathcal{C}(G)^{K^{\perp}}$-modules $\left(G \ltimes \iota_{H}^{K}\right)^{*} X^{K}$ and $X^{H} \otimes_{\mathcal{C}(G)^{H^{\perp}}} R_{H^{\perp}}^{K^{\perp}}$ are isomorphic. Recall that $X^{K}=X^{H}=\mathcal{C}(G)$ and $R_{H^{\perp}}^{K^{\perp}}=\mathcal{C}(G)^{H^{\perp}}$ as vector spaces. A lengthy computation using the description of $X^{K}$ and $X^{H}$ given by the formulas (23.2) - (23.5) and (15.12) shows that

$$
\left(G \ltimes \iota_{H}^{K}\right)^{*} X^{K} \rightarrow X^{H} \otimes_{\mathcal{C}(G)^{H^{\perp}}} R_{H^{\perp}}^{K^{\perp}}, \quad f \mapsto f \otimes_{\mathcal{C}(G)^{H^{\perp}}} \frac{1}{\left|K^{\perp}\right|^{1 / 2}},
$$

is a well-defined isomorphism of Kasparov $G \ltimes \mathcal{C}(G)^{H}-\mathcal{C}(G)^{K^{\perp}}$-modules. This shows $\mathcal{I}\left(\iota_{H}^{K}\right)=\rho_{H^{\perp}}^{K^{\perp}}$. The other equality follows by applying $\mathcal{I}$.

Lemma 15.19. Let $K \leq H \leq L \leq G$. We have $\iota_{L}^{H} \cdot \iota_{H}^{K}=\iota_{L}^{K}$ and $\rho_{K}^{H} \cdot \rho_{H}^{L}=\rho_{K}^{L}$.

Proof. The first equality is obvious, the second one follows by applying $\mathcal{I}$.

In the next lemma, we will show that $\iota_{H}^{K}$ and $\rho_{K}^{H}$ behave nicely under induction. Recall that $\operatorname{Ind}_{H}^{G}$ is a functor from $\mathfrak{K}_{\mathfrak{K}}^{H}$ to $\mathfrak{K}_{\mathfrak{K}}^{G}$. To avoid confusion, we will denote the morphisms $\iota_{H}^{K}$ and $\rho_{K}^{H}$ of $\mathfrak{K} K^{H}$ by $\iota_{H}^{K}(H)$ and $\rho_{K}^{H}(H)$, respectively, and similarly for the corresponding morphisms in $\mathfrak{K} \mathfrak{K}^{G}$.

Lemma 15.20. Let $K \leq H \leq G$. Under the identification

$$
\operatorname{Ind}_{H}^{G} \mathcal{C}(H)^{K} \cong \mathcal{C}(G)^{K}, f \mapsto(g \mapsto f(g, 0))
$$

we have

$$
\operatorname{Ind}_{H}^{G}\left(\iota_{H}^{K}(H)\right)=\iota_{H}^{K}(G) \text { and } \operatorname{Ind}_{H}^{G}\left(\rho_{K}^{H}(H)\right)=\rho_{K}^{H}(G)
$$

Proof. The first equality follows straight from the definition. For the second equality, recall that $\rho_{K}^{H}(H)=\left[R_{K}^{H}(H)\right]$ and that the Kasparov bimodule $R_{K}^{H}(H)$ is equal to $\mathcal{C}(H)^{K}$ as a vector space. The isomorphism $\operatorname{Ind}_{H}^{G} \mathcal{C}(H)^{K} \cong \mathcal{C}(G)^{K}$, regarded as an isomorphism of vector spaces, can be applied to the Kasparov bimodule representing $\operatorname{Ind}_{H}^{G} \rho_{K}^{H}(H)$ and yields an isomorphism of Kasparov $\mathcal{C}(G)^{K}-\mathcal{C}(G)^{H}$-bimodules $R_{K}^{H}(G) \cong$ $\operatorname{Ind}_{H}^{G} R_{K}^{H}(H)$.

Proposition 15.21. Let $K \leq H \leq G$, then

$$
\iota_{H}^{K} \cdot \rho_{K}^{H}=\sum_{g \in K^{\perp} / H^{\perp}} r_{g}^{H} \in \mathrm{KK}^{G}\left(\mathcal{C}(G)^{H}, \mathcal{C}(G)^{H}\right) .
$$

Proof. Note that $\iota_{G}^{\{0\}} \cdot \rho_{\{0\}}^{G} \in \mathrm{KK}^{G}(\mathbb{C}, \mathbb{C})$ is the class of the representation of $G$ on $L^{2}(G)$ via left translation. Hence, by the Peter-Weyl Theorem [32], we have

$$
\iota_{G}^{\{0\}} \cdot \rho_{\{0\}}^{G}=\sum_{\phi \in \widehat{G}}[\phi] .
$$




\section{A UCT for Actions of Finite Cyclic Groups}

Pullback via the quotient map $\pi: H \rightarrow H / K$ yields $\pi^{*}: \mathfrak{K} \mathfrak{K}^{H / K} \rightarrow \mathfrak{K} \mathfrak{K}^{H}$. Applying $\pi^{*}$ to the equality above (for $G=H / K$ ) and using the identification $\mathcal{C}(H / K) \cong \mathcal{C}(H)^{K}$, we obtain

$$
\iota_{H}^{K} \cdot \rho_{K}^{H}=\sum_{\phi \in \widehat{H / K}}[\phi \circ \pi] \in \mathrm{KK}^{H}\left(\mathcal{C}(H)^{H}, \mathcal{C}(H)^{H}\right) .
$$

Applying $\operatorname{Ind}_{H}^{G}$ and Lemma 15.20, we obtain

$$
\iota_{H}^{K} \cdot \rho_{K}^{H}=\sum_{\phi \in \widehat{H / K}} \operatorname{Ind}_{H}^{G}[\phi \circ \pi] \in \mathrm{KK}^{G}\left(\mathcal{C}(G)^{H}, \mathcal{C}(G)^{H}\right) .
$$

The exact sequence $H \longmapsto G \rightarrow G / H$ induces an exact sequence $\widehat{G / H} \longmapsto \widehat{G} \rightarrow \widehat{H}$. Therefore,

$$
\begin{aligned}
& \sum_{\phi \in \widehat{H / K}} \operatorname{Ind}_{H}^{G}[\phi \circ \pi]=\sum_{\psi \in \widehat{H}, \psi_{\mid K}=1} \operatorname{Ind}_{H}^{G}[\psi]=\frac{1}{|\widehat{G / H}|} \sum_{\gamma \in \widehat{G}, \gamma_{\mid K}=1} \operatorname{Ind}_{H}^{G} \operatorname{Res}_{G}^{H}[\gamma] . \\
= & \frac{1}{\left|H^{\perp}\right|} \sum_{g \in K^{\perp}} \operatorname{Ind}_{H}^{G} \operatorname{Res}_{G}^{H}\left[\chi_{g}\right]=\frac{1}{\left|H^{\perp}\right|} \sum_{g \in K^{\perp}}\left[\chi_{g}\right] \otimes_{\mathbb{C}} \mathcal{C}(G)^{H}=\frac{1}{\left|H^{\perp}\right|} \sum_{g \in K^{\perp}} r_{g}^{H} .
\end{aligned}
$$

Corollary 15.22. Let $K \leq H \leq G$, then

$$
\rho_{K}^{H} \cdot \iota_{H}^{K}=\frac{1}{|K|} \sum_{g \in H} t_{g}^{K} \in \mathrm{KK}^{G}\left(\mathcal{C}(G)^{K}, \mathcal{C}(G)^{K}\right) .
$$

Proof. This follows by applying $\mathcal{I}$ to the statement of the last proposition.

\section{Projective Resolutions of Length 1}

From now on let $G$ denote a finite cyclic group $G$ of order $\mathbf{o}$. The aim of this section is to show that every module over $\mathfrak{C}^{G} \otimes \mathbb{Z}\left[\mathbf{o}^{-1}\right]$ has projective dimension 1 .

Let us first give an outline of the argument: For every finite cyclic group $G$, there is a decomposition $G=\bigoplus G_{i}$, where each $G_{i}$ is a finite cyclic group of prime power order $p_{i}^{\epsilon}$. $\mathfrak{C}^{G} \otimes \mathbb{Z}\left[\mathbf{o}^{-1}\right]$ can be decomposed into the tensor product of categories $\mathfrak{C}^{-G_{i}}\left[p_{i}^{-1}\right]$. We will introduce the notion of a split category over a family of rings $\left\{R_{i}\right\}_{i \in I}$, which basically means that the category of modules over this category is equivalent to modules over $\bigoplus_{I} R_{i}$. If every $R_{i}$ is a direct sum of Dedekind domains, modules over $\bigoplus_{I} R_{i}$ have projective dimension 1 . The same is true for modules over a category which is split over $\left\{R_{i}\right\}_{i \in I}$. We will then calculate $\mathfrak{C}^{G_{i}} \otimes \mathbb{Z}\left[p_{i}{ }^{-1}\right]$ explicitly in terms of hand-selected generators and relations to show that it is indeed split over a family of Dedekind domains. Together with the fact that tensor products of split categories are again split, this implies the claim. 


\subsection{Split Categories and Tensor products}

Definition 16.1. Let $\mathfrak{C}$ be a finite preadditive category and $\left\{R_{i}\right\}_{i \in I}$ a finite family of unital commutative rings. Let $1_{i}$ denote the unit in $R_{i}$. $\mathfrak{C}$ is called split over $\left\{R_{i}\right\}_{i \in I}$ if and only if:

(1) For all $A, B \in \in \mathfrak{C}$, there are subsets $I(A, B) \subseteq I$ such that

$$
I(A, B)=I(B, A), I(A, B) \cap I(B, C) \subset I(A, C) \text { and } I=\bigcup_{A \in \in \mathfrak{C}} I(A, A) .
$$

(2) For $A, B \in \in \mathfrak{C}$, there is an isomorphism of abelian groups

$$
\gamma_{A}^{B}: \bigoplus_{i \in I(A, B)} R_{i} \cong \mathfrak{C}(A, B)
$$

(3) If $i \in I(A, B), j \in I(B, C), r \in R_{i}, s \in R_{j}$, then composition in $\mathfrak{C}$ is given by

$$
\gamma_{A}^{B}\left(r 1_{i}\right) \cdot \gamma_{B}^{C}\left(s 1_{j}\right)=\delta_{i, j} \gamma_{A}^{C}\left(r s 1_{i}\right) .
$$

A trivial example of a split category over $\left\{R_{i}\right\}_{i \in I}$ is the category $\mathfrak{C}$ with objects $I$ and morphisms

$$
\mathfrak{C}(i, j):=\left\{\begin{array}{ll}
R_{i} & \text { if } i=j, \\
0 & \text { otherwise. }
\end{array} .\right.
$$

Note that the morphism groups of a finite preadditive category $\mathfrak{C}$, which is split over $\left\{R_{i}\right\}_{i \in I}$ carry a module structure over $R:=\bigoplus_{i \in I} R_{i}:$ For $i \in I, A, B \in \in \mathfrak{C}, j \in I(A, B)$ and $r \in R_{i}, s \in R_{j}$ set

$$
r 1_{i} \cdot \gamma_{A}^{B}\left(s 1_{j}\right):=\delta_{i, j} \gamma_{A}^{B}\left(r s 1_{j}\right) .
$$

This module structure is compatible with composition in the sense that for $A, B, C \in \in \mathfrak{C}$, $r, s \in R$ and $x \in \mathfrak{C}(A, B)$ and $y \in \mathfrak{C}(B, C)$,

$$
(r \cdot x) \cdot(s \cdot y)=(r s) \cdot(x \cdot y) .
$$

Lemma 16.2. Let $\mathfrak{C}$ be split over $\left\{R_{i}\right\}_{i \in I}$ and set $R:=\bigoplus_{I} R_{i}$. Then there are equivalences of categories.

$$
\mathfrak{M o d}(\mathfrak{C}) \simeq \mathfrak{M o d}(R), \quad \mathfrak{M o d}(\mathfrak{C})_{\mathrm{c}} \simeq \mathfrak{M o d}(R)_{\mathrm{c}}
$$

Proof. We have to define additive functors

$$
\mathfrak{M o d}(\mathfrak{C}) \stackrel{F}{\rightarrow} \mathfrak{M o d}(R) \stackrel{G}{\rightarrow} \mathfrak{M o d}(\mathfrak{C})
$$

and show that there are isomorphisms $F \circ G \cong \operatorname{id}_{\mathfrak{M} \mathfrak{o}(R)}$ and $G \circ F \cong \operatorname{id}_{\mathfrak{M o d}(\mathfrak{C})}$. Let us first define $G: \mathfrak{M o d}(R) \rightarrow \mathfrak{M o d}(\mathfrak{C}):$ For $A \in \in \mathfrak{C}$ and $M \in \in \mathfrak{M o d}(R)$, set $G(M)(A):=$ $\bigoplus_{i \in I(A, A)} 1_{i} M$ and for $A, B \in \in \mathfrak{C}, j \in I(A, B)$ and $r \in R_{j}$, let

$$
G(M)\left(\gamma_{A}^{B}\left(r 1_{j}\right)\right): G(M)(A) \rightarrow G(M)(B)
$$




\section{A UCT for Actions of Finite Cyclic Groups}

be given by the composition

$$
G(M)(A)=\bigoplus_{i \in I(A, A)} 1_{i} M \rightarrow 1_{j} M \stackrel{r 1_{j}}{\longrightarrow} 1_{j} M \longmapsto \bigoplus_{i \in I(B, B)} 1_{i} M=G(M)(B) .
$$

It is easy to check that this gives a well-defined additive functor. For the definition of $F$, choose a map $\sigma$, which assigns to $i \in I$ an object $\sigma(i) \in \in \mathfrak{C}$ such that $i \in I(\sigma(i), \sigma(i))$ for all $i \in I$ and define an idempotent

$$
p_{i}:=\gamma_{\sigma(i)}^{\sigma(i)}\left(1_{i}\right) \in \mathfrak{C}(\sigma(i), \sigma(i))
$$

For $N \in \in \mathfrak{M} \mathfrak{o d}(\mathfrak{C})$, let $\Im N\left(p_{i}\right)$ denote the image of $N\left(p_{i}\right): N(\sigma(j)) \rightarrow N(\sigma(j))$ and set

$$
F(N):=\bigoplus_{i \in I} \Im N\left(p_{i}\right) .
$$

For $j \in I$ and $r \in R_{j}$, define multiplication by $r 1_{j}$ by the composition

$$
\bigoplus_{i \in I} \Im N\left(p_{i}\right) \rightarrow \Im N\left(p_{j}\right) \stackrel{N\left(r p_{j}\right)}{\longrightarrow} \Im N\left(p_{j}\right) \longmapsto \bigoplus_{i \in I} \Im N\left(p_{i}\right) .
$$

It is not hard to check that this gives a well-defined additive functor.

For $M \in \in \mathfrak{M o d}(R)$, compute $F \circ G(M)=\bigoplus_{i \in I} \Im G(M)\left(p_{i}\right)=\bigoplus_{i \in I} 1_{i} \cdot M$. Since for every $R$-module $M$, there is a natural isomorphism $M \cong \bigoplus_{i \in I} 1_{i} \cdot M$, we obtain a natural isomorphism $F \circ G \cong \operatorname{id}_{\mathfrak{M o d}(R)}$. For $N \in \in \mathfrak{M o d}(\mathfrak{C})$, we compute $G \circ$ $F(N)(A)=\bigoplus_{i \in I(A, A)} \Im N\left(p_{i}\right)$. For $i \in I(A, A), N\left(\gamma_{A}^{\sigma(i)}\left(1_{i}\right)\right)$ yields an isomorphism $\Im N\left(p_{i}\right) \cong N\left(g_{A}^{A}(i)\right) N(A)$ with inverse $N\left(\gamma_{\sigma(i)}^{A}\left(1_{i}\right)\right)$. This gives a natural isomorphism

$$
\Phi_{N, A}: G \circ F(N)(A)=\bigoplus_{i \in I(A, A)} \Im N\left(p_{i}\right) \cong \bigoplus_{i \in I(A, A)} N\left(g_{A}^{A}(i)\right) N(A) \cong N(A)
$$

We obtain a natural isomorphism $\Phi_{N}:=\left(\Phi_{N, A}\right)_{A \in \in \mathfrak{C}}: G \circ F(N) \cong N$ and $\Phi:=$ $\left(\Phi_{N}\right)_{N \in \in \mathfrak{M o d}(\mathfrak{C})}$ gives the desired natural isomorphism $G \circ F \cong \mathrm{id}_{\mathfrak{M o d}(\mathfrak{C})}$. This shows that $F$ and $G$ implement an equivalence $\mathfrak{M o d}(\mathfrak{C}) \simeq \mathfrak{M o d}(R)$. It is clear that $F$ and $G$ preserve the countability condition. Therefore, we also obtain an equivalence $\mathfrak{M o d}(\mathfrak{C})_{\mathrm{c}} \simeq$ $\mathfrak{M o d}(R)_{\mathrm{c}}$.

Corollary 16.3. Let $\mathfrak{C}$ be split over $\left\{R_{i}\right\}_{i \in I}$ such that every $R_{i}$ is isomorphic to a countable direct sum of countable Dedekind domains. Then every object in $\mathfrak{M o d}(\mathfrak{C})_{\mathrm{c}}$ has a projective resolution of length 1 .

Let $J$ be a finite index set and for $j \in J, \mathfrak{C}_{j}$ a finite preadditive category. In the following, a tensor product without subscript will always denote the algebraic tensor product over $\mathbb{Z}$ (of rings, categories or modules). 
Definition 16.4. Let $\mathfrak{D}$ be a preadditive category. A functor $F: \prod_{J} \mathfrak{C}_{j} \rightarrow \mathfrak{D}$ is called multilinear, if for all $\left(C_{j}\right)_{j \in J},\left(C_{j}^{\prime}\right)_{j \in J} \in \in \prod_{J} \mathfrak{C}_{j}$ the map

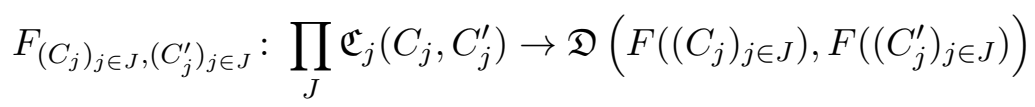

is multilinear over $\mathbb{Z}$.

Definition 16.5. A tensor product of $\left(\mathfrak{C}_{j}\right)_{j \in J}$ is a pair $\left(\bigotimes_{J} \mathfrak{C}_{j}, \bigotimes_{J}\right)$, where $\bigotimes_{J} \mathfrak{C}_{j}$ is a preadditive category and $\bigotimes_{J}: \prod_{J} \mathfrak{C}_{j} \rightarrow \bigotimes_{J} \mathfrak{C}_{j}$ is a multilinear functor such that for every multilinear functor $F: \prod_{J} \mathfrak{C}_{j} \rightarrow \mathfrak{D}$, there is a unique additive functor $\tilde{F}: \bigotimes_{J} \mathfrak{C}_{j} \rightarrow$ $\mathfrak{D}$ such that

$$
\tilde{F} \circ \bigotimes_{J}=F
$$

Lemma 16.6. $\left(\bigotimes_{J} \mathfrak{C}_{j}, \bigotimes_{J}\right)$ exists.

Proof. Let the objects of $\otimes_{J} \mathfrak{C}_{j}$ be equal to the objects of $\prod_{J} \mathfrak{C}_{j}$. For $C_{j} \in \in \mathfrak{C}_{j}, j \in J$ we will write $\bigotimes_{J} C_{j}$ to denote the object $\left(C_{j}\right)_{j \in J}$. Let additionally $D_{j} \in \in \mathfrak{C}_{j}$ for $j \in J$ and define

$$
\bigotimes_{J} \mathfrak{C}_{j}\left(\bigotimes_{J} C_{j}, \bigotimes_{J} D_{j}\right):=\bigotimes_{J} \mathfrak{C}_{j}\left(C_{j}, D_{j}\right)
$$

For $j \in J$, let $C_{j}, D_{j}, E_{j} \in \in \mathfrak{C}_{j}$ and $f_{j} \in \mathfrak{C}_{j}\left(C_{j}, D_{j}\right)$ and $g_{j} \in \mathfrak{C}_{j}\left(D_{j}, E_{j}\right)$. Composition is given by

$$
\bigotimes_{J} f_{j} \cdot \bigotimes_{J} g_{j}:=\bigotimes_{J} f_{j} \cdot g_{j}
$$

It is elementary to check that this product is associative. Let $\bigotimes_{J}: \prod_{J} \mathfrak{C}_{j} \rightarrow \bigotimes_{J} \mathfrak{C}_{j}$ be given by $\left(C_{j}\right)_{j \in J} \mapsto \otimes_{J} C_{j}$ on objects (which is just a change in notation) and by $\left(f_{j}\right)_{j \in J} \mapsto \bigotimes_{J} f_{j}$ on morphisms. It is easy to check that $\left(\bigotimes_{J} \mathfrak{C}_{j}, \bigotimes_{J}\right)$ has the required universal property.

The tensor product is associative up to an isomorphism of categories.

Lemma 16.7. Let $J$ be a finite index set and for $j \in J$ let $\left\{R_{i, j}\right\}_{i \in I_{j}}$ be a finite family of commutative unital rings. For $j \in J$, let $\mathfrak{C}_{j}$ be split over $\left\{R_{i, j}\right\}_{i \in I_{j}}$. Set $I:=\prod_{j \in J} I_{j}$. Then $\bigotimes_{J} \mathfrak{C}_{j}$ is split over $\left\{\bigotimes_{J} R_{i_{j}, j}\right\}_{\left(\left(i_{j}\right)_{j \in J}\right) \in I}$.

Proof. For $A:=\bigotimes_{J} A_{j}, B:=\bigotimes_{J} B_{j} \in \in \bigotimes_{J} \mathfrak{C}_{j}$, define

$$
I(A, B):=\prod_{J} I\left(A_{j}, B_{j}\right)
$$

Define $\gamma_{A}^{B}$ by commutativity of the following diagram

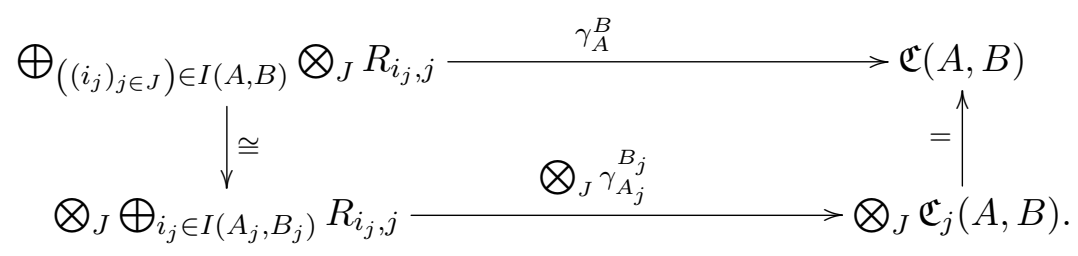




\section{A UCT for Actions of Finite Cyclic Groups}

This endows $\bigotimes_{J} \mathfrak{C}_{j}$ with the structure of a category, which is split over $\left\{\bigotimes_{J} R_{i_{j}, j}\right\}_{\left(i_{j}\right)_{j \in J} \in I}$.

\subsection{A Special Case}

Let us consider the case $G=\mathbf{C}\left(p^{n}\right), n \in \mathbb{N}$ and $p$ prime. Our aim is to show that $\mathfrak{C}^{G}\left[p^{-1}\right]$ is split over a family of Dedekind domains. We will be using facts about cyclotomic polynomials and decompositions of certain rings into Dedekind domains. For better readability, we collected these purely algebraic statements in the appendix, Section 22.

To simplify notation, let $\mathfrak{C}$ denote the category with objects $\{0,1, \ldots, n\}$ and morphisms

$$
\mathfrak{C}(k, l):=\mathrm{KK}^{\mathbf{C}\left(p^{n}\right)}\left(\mathcal { C } \left(\mathbf{C}\left(p^{n}\right)^{\left.\mathbf{C}\left(p^{k}\right)\right)}, \mathcal{C}\left(\mathbf{C}\left(p^{n}\right)^{\left.\mathbf{C}\left(p^{l}\right)\right)}\right)\left[p^{-1}\right] .\right.\right.
$$

$\mathfrak{C}$ is obviously isomorphic to $\mathfrak{C}^{G}\left[p^{-1}\right], G=\mathbf{C}\left(p^{n}\right)$.

\section{Definition 16.8.}

$$
I:=\{(u, v) \mid \exists k, 0 \leq k \leq n \text { such that } 0 \leq u \leq n-k, 0 \leq v \leq k\} .
$$

For $(u, v) \in I$, define

$$
R_{(u, v)}:=\left(\mathbb{Z}\left[p^{-1}\right][t] /\left\langle\Phi_{p^{u}}(t)\right\rangle\right) \otimes\left(\mathbb{Z}\left[p^{-1}\right][r] /\left\langle\Phi_{p^{v}}(r)\right\rangle\right),
$$

where $\Phi_{m}$ denotes the $m$ th cyclotomic polynomial

$$
\Phi_{m}(t):=\prod_{\omega \text { th primitive root of unity }}(t-\omega) .
$$

Having made these definitions, we can be more precise about what we want to prove in this subsection:

Theorem 16.9. $\mathfrak{C}$ is split over $\left\{R_{(u, v)} \mid(u, v) \in I\right\}$.

Note that this implies that all countable $\mathbb{Z} / 2$-graded modules over $\mathfrak{C}$ have a projective resolution of length $1: \mathbb{Z}\left[p^{-1}\right][t] /\left\langle\Phi_{p^{u}}(t)\right\rangle$ is isomorphic to the Dedekind domain $\mathbb{Z}\left[p^{-1}, \theta_{p^{u}}\right]$ by Lemma 22.2 , and Proposition 22.11 yields an isomorphism of rings

$$
R_{(u, v)} \cong \mathbb{Z}\left[p^{-1}, \theta_{p^{u}}\right] \otimes \mathbb{Z}\left[p^{-1}, \theta_{p^{v}}\right] \cong \bigoplus_{0<k<p^{\min (u, v)}, \operatorname{gcd}(p, k)=1} \mathbb{Z}\left[p^{-1}, \theta_{p^{\max (u, v)}}\right] .
$$

Therefore, all objects in $\mathfrak{M o d}(\mathfrak{C})_{\mathrm{c}}^{\mathbb{Z} / 2}$ have projective dimension 1 by Corollary 16.3 .

The verification of conditions (1)-(3) in the definition of a split category or equivalently, the proof of Theorem 16.9 will occupy the remainder of this section.

Definition 16.10. For $0 \leq k, l \leq n$, set

$$
I(k, l):=\{(u, v) \mid 0 \leq u \leq n-k \vee l, 0 \leq v \leq k \wedge l\} .
$$

Here $k \vee l:=\max (k, l)$ and $k \wedge l:=\min (k, l)$. 
It is immediate that $I(k, l)=I(l, k), I(k, l) \cap I(l, m) \subset I(k, m)$ and $I=\bigcup_{k=0, \ldots, n} I(k, k)$. Hence condition (1) in Definition 16.1 holds.

The next step is to define the isomorphisms of abelian groups, which are part of condition (2) in Definition 16.1:

$$
\gamma_{k}^{l}: \bigoplus_{(u, v) \in I(k, l)} R_{(u, v)} \cong \mathfrak{C}(k, l)
$$

$\gamma_{k}^{l}$ will be constructed by using the basic building blocks for generators in $\mathfrak{C}$ (see Definitions 15.9 and 15.14). To further simplify notation, let us make the following definitions

Definition 16.11. Let $e$ denote the class of 1 in $\mathbf{C}\left(p^{n}\right)$. For $0 \leq k, l \leq n$, define

$$
{ }_{k} t_{k}:=t_{e}^{\mathbf{C}\left(p^{k}\right)} \in \mathfrak{C}(k, k), \quad{ }_{k} r_{k}:=r_{e}{ }_{e}^{\mathbf{C}\left(p^{k}\right)} \in \mathfrak{C}(k, k), \quad{ }_{k} \mu_{l}:=\mu_{\mathbf{C}\left(p^{k}\right)}^{\mathbf{C}\left(p^{l}\right)} \in \mathfrak{C}(k, l) .
$$

Define polynomials $\psi_{n, m} \in \mathbb{Z}\left[n^{-1}\right][t]$ by

$$
\psi_{n, m}(t):=\frac{1}{n} \cdot t \cdot \frac{\mathrm{d}}{\mathrm{d} t} \Phi_{m}(t) \cdot \prod_{m^{\prime} \mid n, m^{\prime} \neq m} \Phi_{m^{\prime}}(t) \in \mathbb{Z}\left[n^{-1}\right][t] .
$$

The relevance of the $\psi_{n, m} \mathrm{~s}$ is that they allow for a decomposition of $\mathbb{Z}\left[n^{-1}\right] /\left\langle t^{n}-1\right\rangle$ into a direct sum of Dedekind domains (Proposition 22.8 in the appendix). Let us abbreviate $\psi_{p^{k}, p^{u}}$ by $\Psi_{k, u}$.

Lemma 16.12. Let $k \leq l, u \in \mathbb{N}$ and $x \in \mathfrak{C}(k, l)$. For $0 \leq u \leq n-k$, we have

$$
\Psi_{n-k, u}\left({ }_{k} t_{k}\right) \cdot x= \begin{cases}x \cdot \Psi_{n-l, u}\left({ }_{l} t_{l}\right) & \text { if } u \leq n-l, \\ 0 & \text { otherwise. }\end{cases}
$$

For $0 \leq v \leq l$, we have

$$
x \cdot \Psi_{l, v}\left({ }_{l} r_{l}\right)= \begin{cases}\Psi_{k, v}\left({ }_{k} r_{k}\right) \cdot x & \text { if } v \leq k \\ 0 & \text { otherwise. }\end{cases}
$$

Proof. Note first that ${ }_{k} t_{k} \cdot x=x \cdot{ }_{l} t_{l}$. Hence $\Psi_{n-k, u}\left({ }_{k} t_{k}\right) \cdot x=x \cdot \Psi_{n-k, u}\left({ }_{l} t_{l}\right)$. Since $\left({ }_{k} t_{k}\right)^{p^{n-k}}=1$, the first statement follows by Lemma 22.7. The second statement is proven analogously.

Let us set

$$
c_{k, l}:= \begin{cases}p^{k-l} & \text { if } k \geq l \\ 1 & \text { otherwise. }\end{cases}
$$

Proposition 16.13. Let $0 \leq k, l \leq n$, then there is an isomorphism of abelian groups

$$
\gamma_{k}^{l}: \bigoplus_{(u, v) \in I(k, l)} R_{(u, v)} \cong \mathfrak{C}(k, l)
$$

which is given by

$$
\gamma_{k}^{l}\left(p \otimes q 1_{(u, v)}\right) \mapsto c_{k, l} \cdot p\left({ }_{k} t_{k}\right) \cdot \Psi_{n-k, u}\left({ }_{k} t_{k}\right) \cdot{ }_{k} \mu_{l} \cdot q\left({ }_{l} r_{l}\right) \cdot \Psi_{l, v}\left({ }_{l} r_{l}\right) .
$$




\section{A UCT for Actions of Finite Cyclic Groups}

Here, $p$ and $q$ are polynomials representing elements in $\mathbb{Z}\left[p^{-1}\right][t] /\left\langle\Phi_{p^{u}}(t)\right\rangle$ and $\mathbb{Z}\left[p^{-1}\right][r] /\left\langle\Phi_{p^{v}}(r)\right\rangle$. Proof. Let $0 \leq k, l \leq n$, then

$$
\left\{\left({ }_{k} t_{k}\right)^{a} \cdot{ }_{k} \mu_{l} \cdot\left({ }_{l} r_{l}\right)^{b} \mid 0 \leq a<p^{n-k \vee l}, 0 \leq b<p^{k \wedge l}\right\}
$$

is a basis of $\mathfrak{C}(k, l)$ as a free $\mathbb{Z}\left[p^{-1}\right]$-module by Proposition 15.15. In other words, there is an isomorphism of abelian groups

$$
\mathbb{Z}\left[p^{-1}\right][t] /\left\langle t^{p^{n-k \vee l}}-1\right\rangle \otimes \mathbb{Z}\left[p^{-1}\right][r] /\left\langle r^{p^{k \wedge l}}-1\right\rangle \cong \mathfrak{C}(k, l), \quad p \otimes q \mapsto p\left({ }_{k} t_{k}\right) \cdot{ }_{k} \mu_{l} \cdot q\left({ }_{l} r_{l}\right) .
$$

By Proposition 22.11, for $0 \leq k, l \leq n$, there is an isomorphism

$$
\bigoplus_{(u, v) \in I(k, l)} R_{(u, v)} \cong \mathbb{Z}\left[p^{-1}\right][t] /\left\langle t^{p^{n-k \vee l}}-1\right\rangle \otimes \mathbb{Z}\left[p^{-1}\right][r] /\left\langle r^{p^{k \wedge l}}-1\right\rangle,
$$

which is given by $p \otimes q \mapsto p \cdot \Psi_{n-k \vee l, u} \otimes q \cdot \Psi_{k \wedge l, v}$ for $p \otimes q \in R_{(u, v)}$. We obtain an isomorphism of abelian groups

$$
\tilde{\gamma}: \bigoplus_{(u, v) \in I(k, l)} R_{(u, v)} \cong \mathfrak{C}(k, l)
$$

given by

$$
\tilde{\gamma}(p \otimes q)=p\left({ }_{k} t_{k}\right) \cdot \Psi_{n-k \vee l, u}\left({ }_{k} t_{k}\right) \cdot{ }_{k} \mu_{l} \cdot q\left({ }_{l} r_{l}\right) \cdot \Psi_{k \wedge l, v}\left({ }_{l} r_{l}\right)
$$

for $p \otimes q \in R_{(u, v)}=\mathbb{Z}\left[p^{-1}\right][t] /\left\langle\Phi_{p^{u}}(t)\right\rangle \otimes \mathbb{Z}\left[p^{-1}\right][r] /\left\langle\Phi_{p^{v}}(r)\right\rangle$. Set $\gamma_{k}^{l}:=c_{k, l} \tilde{\gamma}$.

The last step is to show condition (3) in Definition 16.1, i.e., to show the following statement: If $(u, v) \in I(k, l),\left(u^{\prime}, v^{\prime}\right) \in I(l, m), r \in R_{(u, v)}, s \in R_{\left(u^{\prime}, v^{\prime}\right)}$, then composition in $\mathfrak{C}$ is given by

$$
\gamma_{k}^{l}\left(r 1_{(u, v)}\right) \cdot \gamma_{l}^{m}\left(s 1_{\left(u^{\prime}, v^{\prime}\right)}\right)=\delta_{u, u^{\prime}} \delta_{v, v^{\prime}} \gamma_{k}^{m}\left(r s 1_{(u, v)}\right) .
$$

For $p \in \mathbb{Z}\left[p^{-1}\right][t]$ and $x \in \mathfrak{C}(k, l)$, Lemma 15.10 implies

$$
p\left({ }_{k} t_{k}\right) \cdot x=x \cdot p\left({ }_{l} t_{l}\right) \text { and } p\left({ }_{k} r_{k}\right) \cdot x=x \cdot p\left({ }_{l} r_{l}\right) .
$$

Therefore, it is sufficient to show the following slightly weaker statement:

Proposition 16.14. Let $(u, v) \in I(k, l),\left(u^{\prime}, v^{\prime}\right) \in I(l, m)$, then composition in $\mathfrak{C}$ is given by

$$
\gamma_{k}^{l}\left(1_{(u, v)}\right) \cdot \gamma_{l}^{m}\left(1_{\left(u^{\prime}, v^{\prime}\right)}\right)=\delta_{u, u^{\prime}} \delta_{v, v^{\prime}} \gamma_{k}^{m}\left(1_{(u, v)}\right) .
$$

We will prove this proposition by a series of lemmas.

Lemma 16.15. Let $0 \leq k \leq n$ and $(u, v),\left(u^{\prime}, v^{\prime}\right) \in I(k, k)$, then

$$
\gamma_{k}^{k}\left(1_{(u, v)}\right) \cdot \gamma_{k}^{k}\left(1_{\left(u^{\prime}, v^{\prime}\right)}\right)=\delta_{u, u^{\prime}} \delta_{v, v^{\prime}} \gamma_{k}^{k}\left(1_{(u, v)}\right) .
$$


Proof. In the special case $k=l$, the isomorphism of abelian groups

$$
\mathbb{Z}\left[p^{-1}\right][t] /\left\langle t^{p^{n-k}}-1\right\rangle \otimes \mathbb{Z}\left[p^{-1}\right][r] /\left\langle r^{p^{k}}-1\right\rangle \cong \mathfrak{C}(k, k), \quad p \otimes q \mapsto p\left({ }_{k} t_{k}\right) \cdot q\left({ }_{k} r_{k}\right)
$$

from Proposition 15.15 as well as the isomorphism

$$
\bigoplus_{(u, v) \in I(k, l)} R_{(u, v)} \cong \mathbb{Z}\left[p^{-1}\right][t] /\left\langle t^{p^{n-k}}-1\right\rangle \otimes \mathbb{Z}\left[p^{-1}\right][r] /\left\langle r^{p^{k}}-1\right\rangle
$$

from Proposition 22.11 are isomorphisms of rings. Since $\gamma_{k}^{k}$ is defined as the composition of both, $\gamma_{k}^{k}$ is an isomorphism of rings as well. This shows the claim.

Lemma 16.16. Let $0 \leq k \leq l \leq m \leq n,(u, v) \in I(k, l),\left(u^{\prime}, v^{\prime}\right) \in I(l, m)$, then

$$
\gamma_{k}^{l}\left(1_{(u, v)}\right) \cdot \gamma_{l}^{m}\left(1_{\left(u^{\prime}, v^{\prime}\right)}\right)=\delta_{u, u^{\prime}} \delta_{v, v^{\prime}} \gamma_{k}^{m}\left(1_{(u, v)}\right) .
$$

If $(u, v) \in I(m, l),\left(u^{\prime}, v^{\prime}\right) \in I(l, k)$, then

$$
\gamma_{m}^{l}\left(1_{(u, v)}\right) \cdot \gamma_{l}^{k}\left(1_{\left(u^{\prime}, v^{\prime}\right)}\right)=\delta_{u, u^{\prime}} \delta_{v, v^{\prime}} \gamma_{m}^{k}\left(1_{(u, v)}\right) .
$$

Proof. Lemma 15.18 implies that $\mu_{k}^{l} \cdot \mu_{l}^{m}=\mu_{k}^{m}$. Note also that $c_{k, l} c_{l, m}=c_{k, m}$ since $k \leq l \leq m$. Now compute

$$
\begin{aligned}
& \gamma_{k}^{l}\left(1_{(u, v)}\right) \cdot \gamma_{l}^{m}\left(1_{\left(u^{\prime}, v^{\prime}\right)}\right) \\
& =c_{k, m} \cdot \Psi_{n-k, u}\left({ }_{k} t_{k}\right) \cdot \mu_{k}^{l} \cdot \Psi_{l, v}\left({ }_{l} r_{l}\right) \cdot \Psi_{n-l, u^{\prime}}\left({ }_{l} t_{l}\right) \cdot \mu_{l}^{m} \cdot \Psi_{m, v^{\prime}}\left({ }_{m} r_{m}\right) \\
& =c_{k, m} \cdot \mu_{k}^{l} \cdot \Psi_{n-k, u}\left({ }_{l} t_{l}\right) \cdot \Psi_{n-l, u^{\prime}}\left({ }_{l} t_{l}\right) \cdot \Psi_{l, v}\left({ }_{l} r_{l}\right) \cdot \Psi_{m, v^{\prime}}\left({ }_{l} r_{l}\right) \cdot \mu_{l}^{m} \\
& =\delta_{\left(u, u^{\prime}\right)} \cdot \delta_{\left(v, v^{\prime}\right)} \cdot c_{k, m} \cdot \mu_{k}^{l} \cdot \Psi_{n-l, u}\left({ }_{l} t_{l}\right) \cdot \Psi_{l, v}\left({ }_{l} r_{l}\right) \mu_{l}^{m},
\end{aligned}
$$

where the last equality uses Lemma 22.7 and Lemma 22.6. By Corollary 16.12, the last expression is equal to

$$
\begin{aligned}
& \delta_{\left(u, u^{\prime}\right)} \cdot \delta_{\left(v, v^{\prime}\right)} \cdot c_{k, m} \cdot \Psi_{n-k, u}\left({ }_{k} t_{k}\right) \cdot \mu_{k}^{l} \cdot \mu_{l}^{m} \cdot \Psi_{m, v}\left({ }_{m} r_{m}\right) \\
& =\delta_{\left(u, u^{\prime}\right)} \cdot \delta_{\left(v, v^{\prime}\right)} \cdot c_{k, m} \cdot \Psi_{n-k, u}\left({ }_{k} t_{k}\right) \cdot \mu_{k}^{m} \Psi_{m, v}\left({ }_{m} r_{m}\right) \\
& =\delta_{u, u^{\prime}} \delta_{v, v^{\prime}} \gamma_{m}^{k}\left(1_{(u, v)}\right)
\end{aligned}
$$

Lemma 16.17. Let $0 \leq k \leq l \leq n$, then

$$
\mu_{k}^{l} \cdot \mu_{l}^{k}=\prod_{i=n-l+1}^{n-k} \Phi_{p^{i}}\left({ }_{k} t_{k}\right) \text { and } \mu_{l}^{k} \cdot \mu_{l}^{k}=\prod_{i=k+1}^{l} \Phi_{p^{i}}\left({ }_{l} r_{l}\right) .
$$

Proof. By Corollary 15.22, we have

$$
\mu_{k}^{l} \cdot \mu_{l}^{k}=p^{-k} \sum_{i=0}^{p^{l}-1}\left({ }_{k} t_{k}\right)^{i p^{n-l}}
$$


Since $\left({ }_{k} t_{k}\right)^{p^{n-k}}=1$, we obtain

$$
\begin{aligned}
\mu_{k}^{l} \cdot \mu_{l}^{k} & =p^{-k} \sum_{i=0}^{p^{l}-1}\left({ }_{k} t_{k}\right)^{p^{n-l} i}=\sum_{i=0}^{p^{l-k}-1}\left({ }_{k} t_{k}\right)^{p^{n-l} i} \\
& \left.=\prod_{i=1}^{l-k} \Phi_{p^{i}}\left({ }_{k} t_{k}\right)^{p^{n-l}}\right)=\prod_{i=n-l+1}^{n-k} \Phi_{p^{i}}\left({ }_{k} t_{k}\right),
\end{aligned}
$$

where the last two equalities used the explicit description $\Phi_{p^{k}}(t)=\sum_{i=0}^{p-1} t^{p^{k-1}} i$ from Lemma 22.2 and the fact that $\prod_{i=1}^{n} \sum_{j=0}^{p-1} t^{p^{i-1} j}=\sum_{j=0}^{p^{n}-1} t^{i}$. Similarly, one computes with the help of Proposition 15.21 and the relation $\left({ }_{l} r_{l}\right)^{p^{l}}=1$

$$
\begin{aligned}
\mu_{l}^{k} \cdot \mu_{l}^{k} & =p^{l-n} \sum_{i=0}^{p^{n-k}-1}\left({ }_{l} r_{l}\right)^{p^{k} i} \\
& =\sum_{i=0}^{p^{l-k}-1}\left({ }_{l} r_{l}\right)^{p^{k} i}=\prod_{i=k+1}^{l} \Phi_{p^{i}}\left({ }_{l} r_{l}\right) .
\end{aligned}
$$

Lemma 16.18. Let $0 \leq k \leq l \leq n$ and $(u, v) \in I(k, l),\left(u^{\prime}, v^{\prime}\right) \in I(l, k)$, then

$$
\gamma_{k}^{l}\left(1_{(u, v)}\right) \cdot \gamma_{l}^{k}\left(1_{\left(u^{\prime}, v^{\prime}\right)}\right)=\delta_{u, u^{\prime}} \delta_{v, v^{\prime}} \gamma_{k}^{k}\left(1_{(u, v)}\right)
$$

and

$$
\gamma_{l}^{k}\left(1_{\left(u^{\prime}, v^{\prime}\right)}\right) \cdot \gamma_{k}^{l}\left(1_{(u, v)}\right)=\delta_{u, u^{\prime}} \delta_{v, v^{\prime}} \gamma_{l}^{l}\left(1_{(u, v)}\right) .
$$

Proof. First note that by definition of $\Psi_{n-k, u}$, for $0 \leq u^{\prime} \leq n-l$, we have

$$
p^{l-k} \Psi_{n-l, u^{\prime}}\left({ }_{k} t_{k}\right) \cdot \prod_{i=n-l+1}^{n-k} \Phi_{p^{i}}\left({ }_{k} t_{k}\right)=\Psi_{n-k, u^{\prime}}\left({ }_{k} t_{k}\right)
$$

in $\mathfrak{C}(k, k)$. Now compute

$$
\begin{aligned}
& \gamma_{k}^{l}\left(1_{(u, v)}\right) \cdot \gamma_{l}^{k}\left(1_{\left(u^{\prime}, v^{\prime}\right)}\right) \\
& =p^{l-k} \cdot \Psi_{n-k, u}\left({ }_{k} t_{k}\right) \cdot \mu_{k}^{l} \cdot \Psi_{l, v}\left({ }_{l} r_{l}\right) \cdot \Psi_{n-l, u^{\prime}}\left({ }_{l} t_{l}\right) \cdot \mu_{l}^{k} \cdot \Psi_{k, v^{\prime}}\left({ }_{k} r_{k}\right) \\
& =p^{l-k} \cdot \Psi_{n-k, u}\left({ }_{k} t_{k}\right) \cdot \Psi_{n-l, u^{\prime}}\left({ }_{k} t_{k}\right) \cdot \mu_{k}^{l} \cdot \mu_{l}^{k} \cdot \Psi_{l, v}\left({ }_{k} r_{k}\right) \cdot \Psi_{k, v^{\prime}}\left({ }_{k} r_{k}\right) \\
& =\delta_{v, v^{\prime}} p^{l-k} \cdot \Psi_{n-k, u}\left({ }_{k} t_{k}\right) \cdot \Psi_{n-l, u^{\prime}}\left({ }_{k} t_{k}\right) \cdot \mu_{k}^{l} \cdot \mu_{l}^{k} \cdot \Psi_{k, v}\left({ }_{k} r_{k}\right) \\
& =\delta_{v, v^{\prime}} p^{l-k} \cdot \Psi_{n-k, u}\left({ }_{k} t_{k}\right) \cdot \Psi_{n-l, u^{\prime}}\left({ }_{k} t_{k}\right) \cdot \prod_{i=n-l+1}^{n-k} \Phi_{p^{i}}\left({ }_{k} t_{k}\right) \cdot \Psi_{k, v}\left({ }_{k} r_{k}\right) \\
& =\delta_{v, v^{\prime}} \cdot \Psi_{n-k, u}\left({ }_{k} t_{k}\right) \cdot \Psi_{n-k, u^{\prime}}\left({ }_{k} t_{k}\right) \cdot \Psi_{k, v}\left({ }_{k} r_{k}\right) \\
& =\delta_{u, u^{\prime}} \delta_{v, v^{\prime}} \gamma_{k}^{k}\left(1_{(u, v)}\right),
\end{aligned}
$$


where we used Lemma 22.7 and Lemma 22.6 in the third equality. Similarly, using $p^{l-k} \prod_{i=k+1}^{l} \Phi_{p^{i}}\left({ }_{l} r_{l}\right) \cdot \Psi_{k, v^{\prime}}\left({ }_{l} r_{l}\right)=\Psi_{l, v^{\prime}}\left({ }_{l} r_{l}\right)$, one computes

$$
\begin{aligned}
& \gamma_{l}^{k}\left(1_{\left(u^{\prime}, v^{\prime}\right)}\right) \cdot \gamma_{k}^{l}\left(1_{(u, v)}\right) \\
& =p^{l-k} \cdot \Psi_{n-l, u^{\prime}}\left({ }_{l} t_{l}\right) \cdot \mu_{l}^{k} \cdot \Psi_{k, v^{\prime}}\left({ }_{k} r_{k}\right) \cdot \Psi_{n-k, u}\left({ }_{k} t_{k}\right) \cdot \mu_{k}^{l} \cdot \Psi_{l, v}\left({ }_{l} r_{l}\right) \\
& =\delta_{u, u^{\prime}} \cdot p^{l-k} \cdot \Psi_{n-l, u}\left({ }_{l} t_{l}\right) \mu_{l}^{k} \cdot \mu_{k}^{l} \cdot \Psi_{k, v^{\prime}}\left({ }_{l} r_{l}\right) \cdot \Psi_{l, v}\left({ }_{l} r_{l}\right) \\
& =\delta_{u, u^{\prime}} \cdot p^{l-k} \cdot \Psi_{n-l, u}\left({ }_{l} t_{l}\right) \cdot \prod_{i=k+1}^{l} \Phi_{p^{i}}\left({ }_{l} r_{l}\right) \cdot \Psi_{k, v^{\prime}}\left({ }_{l} r_{l}\right) \cdot \Psi_{l, v}\left({ }_{l} r_{l}\right) \\
& =\delta_{u, u^{\prime}} \delta_{v^{\prime}, v} \cdot \Psi_{n-l, u}\left({ }_{l} t_{l}\right) \cdot \Psi_{l, v}\left({ }_{l} r_{l}\right) \\
& =\delta_{u, u^{\prime}} \delta_{v^{\prime}, v} \gamma_{l}^{l}\left(1_{(u, v)}\right) .
\end{aligned}
$$

Now we are able to prove Proposition 16.14. Let us first recall the statement:

Let $0 \leq k, l, m \leq n$ and $(u, v) \in I(k, l),\left(u^{\prime}, v^{\prime}\right) \in I(l, m)$, then composition in $\mathfrak{C}$ is given by

$$
\gamma_{k}^{l}\left(1_{(u, v)}\right) \cdot \gamma_{l}^{m}\left(1_{\left(u^{\prime}, v^{\prime}\right)}\right)=\delta_{u, u^{\prime}} \delta_{v, v^{\prime}} \gamma_{k}^{m}\left(1_{(u, v)}\right)
$$

Proof. After ordering $k, l$ and $m$, there are six cases to consider:

1. $k \leq l, l \leq m$;

2. $k \leq l, k \leq m \leq l$;

3. $k \leq l, m \leq k$;

4. $l \leq k, m \leq l$;

5. $l \leq k, l \leq m \leq k$;

6. $l \leq k, l \leq m$.

These cases are not mutually exclusive but still exhaustive. We already dealt with cases (1) and (4) in Lemma 16.16. Consider case (2): Since $k \leq m \leq l$, we know that $I(k, m) \cap I(m, l)=I(k, l)$. Using Lemma 16.16 and Lemma 16.18, one computes

$$
\begin{aligned}
\gamma_{k}^{l}\left(1_{(u, v)}\right) \cdot \gamma_{l}^{m}\left(1_{\left(u^{\prime}, v^{\prime}\right)}\right) & =\gamma_{k}^{m}\left(1_{(u, v)}\right) \cdot \gamma_{m}^{l}\left(1_{(u, v)}\right) \cdot \gamma_{l}^{m}\left(1_{\left(u^{\prime}, v^{\prime}\right)}\right) \\
& =\delta_{u, u^{\prime}} \delta_{v, v^{\prime}} \gamma_{k}^{m}\left(1_{(u, v)}\right) \cdot \gamma_{m}^{m}\left(1_{(u, v)}\right) \\
& =\delta_{u, u^{\prime}} \delta_{v, v^{\prime}} \gamma_{k}^{m}\left(1_{(u, v)}\right) .
\end{aligned}
$$

Similarly, we compute in case (3)

$$
\begin{aligned}
\gamma_{k}^{l}\left(1_{(u, v)}\right) \cdot \gamma_{l}^{m}\left(1_{\left(u^{\prime}, v^{\prime}\right)}\right) & =\gamma_{k}^{l}\left(1_{(u, v)}\right) \cdot \gamma_{l}^{k}\left(1_{\left(u^{\prime}, v^{\prime}\right)}\right) \cdot \gamma_{k}^{m}\left(1_{\left(u^{\prime}, v^{\prime}\right)}\right) \\
& =\delta_{u, u^{\prime}} \delta_{v, v^{\prime}} \gamma_{k}^{k}\left(1_{(u, v)}\right) \cdot \gamma_{k}^{m}\left(1_{\left(u^{\prime}, v^{\prime}\right)}\right) \\
& =\delta_{u, u^{\prime}} \delta_{v, v^{\prime}} \gamma_{k}^{m}\left(1_{(u, v)}\right) .
\end{aligned}
$$

Case (5) and case (6) can be dealt with in a completely analogous manner. 


\section{A UCT for Actions of Finite Cyclic Groups}

\subsection{The General Case}

In this subsection, we will show that for a finite cyclic group $G$ of order o, every graded countable module over $\mathfrak{C}^{G}\left[\mathbf{o}^{-1}\right]$ has projective dimension 1.

We will do so by showing that $\mathfrak{C}^{G}\left[\mathbf{o}^{-1}\right]$ is the tensor product of split categories over a family of Dedekind domains and thereby itself is split over a family Dedekind domains.

Let $G$ be a finite cyclic group, then

$$
G=\oplus_{i=1, \ldots, n} G_{i}
$$

with $G_{i}$ cyclic of prime power order $p_{i}^{\epsilon_{i}}$ and $p_{i} \neq p_{j}$ for $i \neq j$. Every subgroup $H$ of $G$ decomposes as $H=\bigoplus_{i} H_{i}$, with $H_{i} \leq G_{i}$.

The next theorem shows that $\mathfrak{C}^{G}$ is isomorphic to $\bigotimes_{i=1}^{n} \mathfrak{C}^{G_{i}}$.

Theorem 16.19. Let $G_{i}, i=1, \ldots, n$ be finite abelian groups and $G:=\bigoplus_{i} G_{i}$. For each $i$, let $\mathfrak{G}_{i}$ be a class of subgroups of $G_{i}$. Define $\mathfrak{G}$ to be the class of subgroups of $G$ of the form $H=\bigoplus_{i} H_{i}$ for $H_{i} \in \mathfrak{G}_{i}$. Let $\mathfrak{C}$ be the full subcategory of $\mathfrak{K} \mathfrak{K}^{G}$ with objects $\mathcal{C}(G)^{H}$, $H \in \mathfrak{G}$. Analogously let $\mathfrak{C}_{i}$ be the full subcategory of $\mathfrak{K} \mathfrak{K}^{G_{i}}$ with objects $\mathcal{C}\left(G_{i}\right)^{H_{i}}, H_{i} \in \mathfrak{G}_{i}$. Then there is an isomorphism

$$
\bigotimes_{i=1}^{n} \mathfrak{C}_{i} \cong \mathfrak{C}
$$

Proof. Let us show the statement for the case $n=2$, the general case follows by induction. We have to define

$$
F: \mathfrak{C}_{1} \otimes \mathfrak{C}_{2} \rightarrow \mathfrak{C}
$$

Note that pullback via the projections $\pi_{i}: G \rightarrow G_{i}$ induces additive functors $\pi_{i}^{*}: \mathfrak{K}^{\mathfrak{K}^{G_{i}}} \rightarrow$ $\mathfrak{K} \mathfrak{K}^{G}$. Restriction to $\mathfrak{C}_{i}$ and taking the tensor product yields

$$
\pi_{1}^{*} \otimes \pi_{2}^{*}: \mathfrak{C}_{1} \otimes \mathfrak{C}_{2} \rightarrow \mathfrak{K K}^{G} \otimes \mathfrak{K K}^{G}
$$

On the other hand, the exterior tensor product in $\mathfrak{K} \mathfrak{K}^{G}$ induces

$$
T: \mathfrak{K} \mathfrak{K}^{G} \otimes \mathfrak{K} \mathfrak{K}^{G} \rightarrow \mathfrak{K} \mathfrak{K}^{G} .
$$

We would like to define $F$ as the composition of the last two functors, but we cannot quite do so since the target objects only match up to a natural isomorphism: For $\mathcal{C}\left(G_{1}\right)^{H_{1}} \otimes$ $\mathcal{C}\left(G_{2}\right)^{H_{2}} \in \in \mathfrak{C}_{1} \otimes \mathfrak{C}_{2}$ and $H:=H_{1} \oplus H_{2}$, there is a natural isomorphism

$$
\Psi_{H}: \mathcal{C}(G)^{H} \cong \pi_{1}^{*} \mathcal{C}\left(G_{1}\right)^{H_{1}} \otimes_{\mathbb{C}} \pi_{1}^{*} \mathcal{C}\left(G_{2}\right)^{H_{2}}
$$

where on the right-hand-side we have the exterior products in $\mathfrak{K} \mathfrak{K}^{G}$, which on the object level is just the spatial tensor product of $C^{*}$-algebras with the diagonal action.

Let us define $F$ on objects by

$$
F\left(\mathcal{C}\left(G_{1}\right)^{H_{1}} \otimes \mathcal{C}\left(G_{2}\right)^{H_{2}}\right):=\mathcal{C}(G)^{H}, \quad H_{i} \in \mathfrak{G}_{i}, H=H_{1} \oplus H_{2}
$$


For $H_{i}, K_{i} \in \mathfrak{G}_{i}, H=H_{1} \oplus H_{2}$ and $K=K_{1} \oplus K_{2}$, define $F$ on morphisms by

$$
\begin{gathered}
\left.F_{H, K}: \mathfrak{C}_{1} \otimes \mathfrak{C}_{2}\left(\mathcal{C}\left(G_{1}\right)^{H_{1}} \otimes \mathcal{C}\left(G_{2}\right)^{H_{2}}, \mathcal{C}\left(G_{1}\right)^{K_{1}} \otimes \mathcal{C}\left(G_{2}\right)^{K_{2}}\right)\right) \rightarrow \mathfrak{C}\left(\mathcal{C}(G)^{H}, \mathcal{C}(G)^{K}\right) \\
F_{H, K}(x)=\left[\Psi_{H}\right] \cdot\left(T \circ\left(\pi_{1}^{*} \otimes \pi_{2}^{*}\right)(x)\right) \cdot\left[\Psi_{K}\right]^{-1} .
\end{gathered}
$$

$F$ is a functor and clearly a bijection on the set of objects. Hence, to verify that $F$ is an isomorphism, we only have to check that each $F_{H, K}$ is an isomorphism. By Proposition 15.15, both domain and target of $F_{H, K}$ are free abelian groups, hence it suffices to check that $F_{H, K}$ maps generators to generators. By definition, the domain of $F_{H, K}$ is equal to

$$
\mathrm{KK}^{G_{1}}\left(\mathcal{C}\left(G_{1}\right)^{H_{1}}, \mathcal{C}\left(G_{1}\right)^{K_{1}}\right) \otimes \mathrm{KK}^{G_{2}}\left(\mathcal{C}\left(G_{2}\right)^{H_{2}}, \mathcal{C}\left(G_{2}\right)^{K_{2}}\right)
$$

and the target is given by

$$
\mathrm{KK}^{G}\left(\mathcal{C}(G)^{H}, \mathcal{C}(G)^{K}\right) .
$$

The following three statements are easily verified using the definitions of $t, r$ and $\mu$ : Let $g_{i} \in G_{i}$, then

1. $F_{H, H}\left(t_{g_{1}}^{H_{1}} \otimes t_{g_{2}}^{H_{2}}\right)=\left[\Psi_{H}\right] \cdot\left(\pi_{1}^{*} t_{g_{1}}^{H_{1}} \otimes_{\mathbb{C}} \pi_{2}^{*} t_{g_{2}}^{H_{2}}\right) \cdot\left[\Psi_{H}\right]^{-1}=t_{\left(g_{1}, g_{2}\right)}^{H_{1} \oplus H_{2}}$

2. $F_{H, H}\left(r_{g_{1}}^{H_{1}} \otimes r_{g_{2}}^{H_{2}}\right)=\left[\Psi_{H}\right] \cdot\left(\pi_{1}^{*} r_{g_{1}}^{H_{1}} \otimes \mathbb{C} \pi_{2}^{*} r_{g_{2}}^{H_{2}}\right) \cdot\left[\Psi_{H}\right]^{-1}=r_{\left(g_{1}, g_{2}\right)}^{H_{1} \oplus H_{2}}$

3. $F_{H, K}\left(\mu_{H_{1}}^{K_{1}} \otimes \mu_{H_{2}}^{K_{2}}\right)=\left[\Psi_{H}\right] \cdot\left(\pi_{1}^{*} \mu_{H_{1}}^{K_{1}} \otimes_{\mathbb{C}} \pi_{2}^{*} \mu_{H_{2}}^{K_{2}}\right) \cdot\left[\Psi_{K}\right]^{-1}=\mu_{H_{1} \oplus H_{2}}^{K_{1} \oplus K_{2}}$.

This, together with the description of the generators of domain and target of $F_{H, K}$ given by Proposition 15.15 , tells us that $F_{H, K}$ maps generators to generators and finishes the proof.

Finally, we will show that every countable graded $\mathfrak{C}^{G}\left[\mathbf{o}^{-1}\right]$-module has a projective resolution of length 1 . First we prove an auxiliary lemma:

Lemma 16.20. Let $\mathfrak{C}$ and $\mathfrak{D}$ be equivalent finite preadditive graded categories. If all objects in $\mathfrak{M o d}(\mathfrak{C})_{\mathrm{c}}^{\mathbb{Z} / 2}$ have projective dimension 1 , then the same holds for all objects in $\mathfrak{M o d}(\mathfrak{D})_{\mathrm{c}}^{\mathbb{Z} / 2}$.

Proof. Let $\alpha: \mathfrak{C} \rightarrow \mathfrak{D}$ and $\beta: \mathfrak{D} \rightarrow \mathfrak{C}$ be grading preserving additive functors such that there are isomorphisms $T: \alpha \circ \beta \cong \operatorname{id}_{\mathfrak{D}}$ and $S: \beta \circ \alpha \cong \mathrm{id}_{\mathfrak{D}}$. Let $M$ be a $\mathfrak{D}$-module, then $\alpha^{*}(M):=M \circ \alpha$ is a $\mathfrak{C}$-module. We obtain preadditive functors

$$
\beta^{*}: \mathfrak{M o d}(\mathfrak{C})_{\mathrm{c}}^{\mathbb{Z} / 2} \rightarrow \mathfrak{M o d}(\mathfrak{D})_{\mathrm{c}}^{\mathbb{Z} / 2} \text { and } \alpha^{*}: \mathfrak{M o d}(\mathfrak{C})_{\mathrm{c}}^{\mathbb{Z} / 2} \rightarrow \mathfrak{M o d}(\mathfrak{C})_{\mathrm{c}}^{\mathbb{Z} / 2}
$$

$T$ and $S$ induce isomorphisms $\alpha^{*} \circ \beta^{*} \cong$ id and $\beta^{*} \circ \alpha^{*} \cong$ id. $\alpha^{*}$ and $\beta^{*}$ are exact and preserve the property of being projective.

Theorem 16.21. Let $G$ be finite cyclic group of order $\mathbf{o}^{-1}$. Then every countable graded $\mathfrak{C}^{G}\left[\mathbf{o}^{-1}\right]$-module has a projective resolution of length 1. 


\section{A UCT for Actions of Finite Cyclic Groups}

Proof. Note that we can ignore the grading: $\mathfrak{C}^{G}\left[\mathbf{o}^{-1}\right]$ is ungraded, therefore, the category of (countable) graded $\mathfrak{C}^{G}\left[\mathbf{o}^{-1}\right]$-modules is the direct sum of two copies of the category of (countable) $\mathfrak{C}^{G}\left[\mathbf{o}^{-1}\right]$-modules. Let $G=\oplus_{i=1, \ldots, n} G_{i}$ with $G_{i}$ cyclic of prime power order $p_{i}^{\epsilon_{i}}$ and $p_{i} \neq p_{j}$ for $i \neq j$. In Theorem 16.19, we showed that $\mathfrak{C}^{G}$ is isomorphic to $\bigotimes_{i=1}^{n} \mathfrak{C}^{G_{i}}$. Since $\mathbf{o}=\prod\left|G_{i}\right|=\prod p_{i}^{\epsilon_{i}}$, we have $\mathbb{Z}\left[\mathbf{o}^{-1}\right]=\bigotimes_{i=1}^{n} \mathbb{Z}\left[p_{i}^{-1}\right]$, this implies that $\mathfrak{C}^{G, \Phi}\left[\mathbf{o}^{-1}\right]$ is isomorphic to $\bigotimes_{i=1}^{n} \mathfrak{C}^{G_{i}}\left[p_{i}^{-1}\right]$. Hence it suffices to show that countable $\bigotimes_{i=1}^{n} \mathfrak{C}^{G_{i}}\left[p_{i}^{-1}\right]$-modules have a projective resolution of length 1 . By Theorem 16.9, $\mathfrak{C}^{G_{i}}\left[p_{i}^{-1}\right]$ is split over

$$
\left\{\mathbb{Z}\left[p_{i}^{-1}, \theta_{p_{i}^{u}}\right] \otimes \mathbb{Z}\left[p_{i}^{-1}, \theta_{p_{i}^{v}}\right]\right\}_{\left\{(u, v) \in \mathbb{N}_{0} \times \mathbb{N}_{0} \mid \exists k \in \mathbb{N}_{0}: k \leq \epsilon_{i}, u \leq \epsilon_{i}-k, v \leq k\right\}} .
$$

Therefore, Lemma 16.7 shows that $\bigotimes_{i=1}^{n} \mathfrak{C}^{G_{i}}\left[p_{i}^{-1}\right]$ is split over

$$
\left\{\bigotimes_{i=1}^{n} \mathbb{Z}\left[p_{i}^{-1}, \theta_{p_{i}^{u_{i}}}\right] \otimes \mathbb{Z}\left[p_{i}^{-1}, \theta_{p_{i}^{v_{i}}}\right]\right\}_{\left\{\left(u_{i}, v_{i}\right)_{i=1, \ldots, n} \in\left(\mathbb{N}_{0} \times \mathbb{N}_{0}\right)^{n} \mid \forall i \exists k_{i} \in \mathbb{N}_{0}: k_{i} \leq \epsilon_{i}, u_{i} \leq \epsilon_{i}-k_{i}, v_{i} \leq k_{i}\right\}} .
$$

In the appendix, we prove the following two facts:

1. Lemma 22.9: Let $m, n \in \mathbb{Z}$ be coprime. Then there is an isomorphism of rings

$$
\mathbb{Z}\left[\theta_{n}\right] \otimes \mathbb{Z}\left[\theta_{m}\right] \cong \mathbb{Z}\left[\theta_{m n}\right]
$$

2. Proposition 22.11: Let $m, n \in \mathbb{N}, n \leq m$ and $p$ a prime number. Then there is an isomorphism of rings

$$
\mathbb{Z}\left[p^{-1}, \theta_{p^{n}}\right] \otimes \mathbb{Z}\left[p^{-1}, \theta_{p^{m}}\right] \cong \bigoplus_{0<k<p^{n}, \operatorname{gct}(p, k)=1} \mathbb{Z}\left[p^{-1}, \theta_{p^{m}}\right] .
$$

Both statements together imply that $\bigotimes_{i=1}^{n} \mathbb{Z}\left[p_{i}^{-1}, \theta_{p^{u_{i}}}\right] \otimes \mathbb{Z}\left[p_{i}^{-1}, \theta_{p^{v_{i}}}\right]$ is isomorphic to a finite direct sum of countable Dedekind domains. Hence Corollary 16.3 shows that every object in $\mathfrak{M o d}\left(\bigotimes_{i=1}^{n} \mathfrak{C}^{G_{i}}\left[p_{i}^{-1}\right]\right)_{\mathrm{c}}$ has a projective resolution of length 1 . 


\section{Universal Coefficient Theorems for $C^{*}$-algebras over Topological Spaces}

The aim of this chapter is to describe explicitly the class of finite topological spaces for which there is a UCT short exact sequence, which computes $\mathrm{KK}(X ; A, B)$ in terms of the filtrated K-theory of $A$ and $B$.

We will first review the notion of a $C^{*}$-algebra over a (possibly non-Hausdorff) finite topological space $X$ and give an alternative description of $X$ by a finite directed graph in Section 17. In Section 18 we will explain how KK-theory for $C^{*}$-algebras over $X$ can be interpreted as a triangulated category with countable coproducts, introduce filtrated K-theory and show that this invariant arises as a special case of our general construction. We will then review alternative characterizations of the bootstrap class and state the UCT criterion in Section 19. Then, in Section 20, we will define spaces of type $A$ and review some spaces, for which there is no UCT in terms of filtrated K-theory available. Finally, in Section 21, we will show that spaces of type $A$ are indeed the most general type of finite topological spaces, for which there is a short exact UCT sequence, which computes $\operatorname{KK}(X, \ldots, \ldots)$ in terms of filtrated K-theory.

\section{$17 C^{*}$-algebras over Finite Topological Spaces}

\subsection{Basic Notions}

Throughout this chapter, let $X$ be a finite topological $T_{0}$ space. A $C^{*}$-algebra over $X$ is a pair $(A, \psi)$ consisting of a $C^{*}$-algebra $A$ and a continuous map $\psi: \operatorname{Prim}(A) \rightarrow X$.

Let $\mathbb{O}(X)$ denote the set of open subsets of $X$, partially ordered by $\subseteq$ and let $\mathbb{I}(A)$ be the set of closed *-ideals in $A$, partially ordered by $\subseteq$. $(\mathbb{O}(X), \subseteq)$ and $(\mathbb{I}(A), \subseteq)$ are complete lattices, that is, any subset $S$ has both an infimum $\bigwedge S$ and a supremum $\bigvee S$. $\psi$ induces a map $\psi^{*}: \mathbb{O}(X) \rightarrow \mathbb{I}(A)$, which commutes with infima and suprema. By [30], Lemma 2.25, $\psi$ can be recovered from $\psi^{*}$. Hence we obtain an equivalent description of a $C^{*}$-algebra over $X$ as a pair $\left(A, \psi^{*}\right)$ where

$$
\psi^{*}: \mathbb{O}(X) \rightarrow \mathbb{I}(A), \quad U \mapsto A(U),
$$

commutes with infima and suprema.

A *-homomorphism $f: A \rightarrow B$ between two $C^{*}$-algebras over $X$ is $X$-equivariant if $f(A(U)) \subseteq B(U)$ for all $U \in \mathbb{O}(X)$. A subset $Y \subseteq X$ is locally closed if and only if $Y=U \backslash V$ for open subsets $V, U \in \mathbb{O}(X)$ with $V \subseteq U$. We define $A(Y):=A(U) / A(V)$ for a $C^{*}$-algebra $A$ over $X$; this does not depend on the choice of $U$ and $V$ by [30] Lemma 2.16 . 
We adopt the following notations from [30].

$\mathbb{O}(X)$ set of open subsets of $X$, partially ordered by $\subseteq$;

$\mathbb{L} \mathbb{C}(X)$ set of locally closed subsets of $X$;

$\mathbb{L} \mathbb{C}(X)_{c}$ set of connected, non-empty locally closed subsets of $X$;

$(A, \psi) C^{*}$-algebra over $X$

$A(Y)$ the subquotient of $(A, \psi)$ associated with $Y \in \mathbb{L} \mathbb{C}(X)$;

$\operatorname{Prim}(A)$ primitive ideal space of $A$ with hull-kernel topology;

$\mathbb{I}(A)$ set of closed *-ideals in $A$, partially ordered by $\subseteq$;

$\mathfrak{C}^{*} \mathfrak{a l g}(X)$ category of $C^{*}$-algebras over $X$ with $X$-equivariant ${ }^{*}$-homomorphisms

$\mathfrak{C}^{*} \mathfrak{s e p}(X)$ full subcategory of separable $C^{*}$-algebras over $X$.

\subsection{Functoriality}

A continuous map $f: X \rightarrow Y$ induces a functor

$$
f_{*}: \mathfrak{C}^{*} \mathfrak{a l g}(X) \rightarrow \mathfrak{C}^{*} \mathfrak{a l g} \mathfrak{g}(Y) .
$$

$f_{*}$ is given by $(A, \psi) \mapsto(A, f \circ \psi)$. We have $g_{*} f_{*}=(g f)_{*}$ whenever it makes sense. If $f: X \rightarrow Y$ is the embedding of a subset with the subspace topology, we also write $i_{X}^{Y}$ instead of $f_{*}$ and call it induction. $Y \in \mathbb{L} \mathbb{C}(X)$ induces a restriction functor

$$
r_{X}^{Y}: \mathfrak{C}^{*} \mathfrak{a l g}(X) \rightarrow \mathfrak{C}^{*} \mathfrak{a l g}(Y)
$$

which is given by $r_{X}^{Y} B(Z):=B(Z)$ for all $Z \in \mathbb{L} \mathbb{C}(Y) \subseteq \mathbb{L} \mathbb{C}(X)$. We have $r_{Y}^{Z} \circ r_{X}^{Y}=r_{X}^{Z}$ if $Z \subseteq Y \subseteq X$ and $r_{X}^{X}=$ id.

Induction and restriction are related by $r_{X}^{Y} \circ i_{Y}^{X}=\mathrm{id}$ and various adjointness properties (see [30] Definition 2.19 and Lemma 2.20 for a discussion of induction and restriction).

\subsection{Specialization Order}

There is the specialization preorder on $X$, defined by $x \preceq y \Longleftrightarrow \overline{\{x\}} \subseteq \overline{\{y\}}$. A subset $Y \subseteq X$ is locally closed if and only if it is convex w.r.t. $\preceq$, i.e., if and only if

$$
x \preceq y \preceq z, x, z \in Y \Rightarrow y \in Y
$$

holds.

Let $X$ be a space and $Y \subseteq X$, there is a locally closed hull, defined as

$$
L C(Y):=\left\{x \in X \mid \exists y_{1}, y_{2} \in Y: y_{1} \preceq x \preceq y_{2}\right\} .
$$


Lemma 17.1. $L C(L C(Y))=L C(Y) . L C(Y)$ is the smallest locally closed set containing $Y$.

Proof. Obviously $Y \subseteq L C(Y)$, let $y \in L C(L C(Y))$, then there are $y_{1}, y_{2} \in L C(Y)$ such that $y_{1} \preceq y \preceq y_{2}$. By definition, there are $z_{1}, z_{2}, z_{3}, z_{4} \in Y$ such that $z_{1} \preceq y_{1} \preceq z_{2}$, $z_{3} \preceq y_{2} \preceq z_{4}$, hence $z_{1} \preceq y_{1} \preceq y \preceq y_{2} \preceq z_{4}$, therefore, $y \in Y$. Using the characterization of locally closed sets as convex sets, the second statement is obvious.

A map $f: X_{1} \rightarrow X_{2}$ between two finite topological spaces is continuous if and only if it is monotone with respect to $\preceq$, i.e. if

$$
x \preceq y \Rightarrow f(x) \preceq f(y)
$$

holds. Note that $\preceq$ is a partial order if and only if $X$ is $T_{0}$. By [30] 2.33, this yields a bijection of partial orders and $T_{0}$-topologies on a given finite set. Let us denote the topology associated with $\prec$ by $\tau_{\prec}$.

\subsection{Representation of Finite Topological Spaces as Directed Graphs}

A useful way to represent finite partially ordered sets and hence finite $T_{0}$ spaces is via finite directed acyclic graphs.

To establish notation, we have collected a few elementary notions of graph theory: A directed graph is a pair $\Gamma=(V, E)$, where $V$ is a set and $E \subseteq V \times V \backslash \Delta(V)$. Elements of $V$ are called vertices and elements of $E$ are called edges. We will also write $E(\Gamma)$ and $V(\Gamma)$ to denote the edges and vertices associated with $\Gamma$. Hence we are neither allowing loops nor multiple edges to exist. A graph $\left(V^{\prime}, E^{\prime}\right)$ is a subgraph of $(V, E)$ if and only if $V^{\prime} \subseteq V$ and $E^{\prime}=\left\{(a, b) \in E \mid a, b \in V^{\prime}\right\}$. A directed path $\rho$ is a a sequence $\rho=\left(v_{i}\right)_{i=0, \ldots, n}$ such that $\left(v_{i}, v_{i+1}\right) \in E$ for $i=1, \ldots, n$ with all $\left(v_{i}\right)_{i=1, \ldots, n}$ being pairwise distinct, the length of $\rho=\left(v_{i}\right)_{i=0, \ldots, n}$ is $n$. A directed circle is a directed path of length larger than 0 such that $v_{0}=v_{n}$. For two paths $\rho_{1}=\left(v_{i}\right)_{i=0, \ldots, n}$ and $\rho_{2}=\left(w_{i}\right)_{i=0, \ldots, m}$, we define sets

$$
\rho_{1} \cap \rho_{2}:=\left\{v_{i} \mid i=0, \ldots, n\right\} \cap\left\{w_{i} \mid i=0, \ldots, m\right\}
$$

and

$$
\rho_{1} \cup \rho_{2}:=\left\{v_{i} \mid i=0, \ldots, n\right\} \cup\left\{w_{i} \mid i=0, \ldots, m\right\} .
$$

An edge $\left(v_{0}, v_{1}\right)$ is called an outgoing edge of $v_{0}$ and an incoming edge of $v_{1}$. The oriented degree $d_{o}(v)$ of $v \in V$ is defined via

$$
d_{o}(v):=\#\{e \in E \mid e \text { outgoing edges of } v\}-\#\{e \in E \mid e \text { incoming edges of } v\}
$$

A directed graph is called acyclic if it has no circles. A path is a sequence $\left(v_{i}\right)_{i=0, \ldots, n}$ such that for $i=1, \ldots, n\left(v_{i}, v_{i+1}\right) \in E$ or $\left(v_{i+1}, v_{i}\right) \in E$ with all $\left(v_{i}\right)_{i=1, \ldots, n}$ being pairwise distinct. We say that $\rho$ is a path from a to $b$ if $v_{0}=a$ and $v_{n}=b$. A circle is a path $\rho=\left(v_{i}\right)_{i=0, \ldots, n}$ of length greater than 0 such that $v_{0}=v_{n}$. The degree $d(v)$ of $v \in V$ is defined as

$$
d(v):=\#\{e \in E \mid e \text { outgoing edge of } v\}+\#\{e \in E \mid e \text { incoming edge of } v\}
$$

With a partial order $\preceq$ on $X$, we associate a finite directed acyclic graph $\Gamma(X)$. 
Definition 17.2. Let $X$ be a finite $T_{0}$ space. Let $\Gamma(X)$ be the directed graph with vertex set $X$ and with an edge $x \leftarrow y$ if and only if $x \prec y$ and there is no $z \in X$ with $x \prec z \prec y$.

We can recover the partial order from this graph by letting $x \preceq y$ if and only if the graph contains a directed path from $y$ to $x$. Note that we cannot obtain every finite directed graph in this way. Although the statements are elementary, we will list the restrictions on $\Gamma(X)$ for later reference.

Lemma 17.3. Let $X$ be a finite $T_{0}$ space. $\Gamma(X)$ is acyclic as a directed graph. Let $x, y$ be vertices in $\Gamma(X)$. If $\rho_{1}$ and $\rho_{2}$ are two distinct directed paths from $x$ to $y$, then $\rho_{1}$ and $\rho_{2}$ have length $\geq 2$.

Proof. Follows straight from the definition.

Let $S$ be a finite set. If $\Gamma$ is a directed graph with vertex set $S$, then we can define a preorder on $S$ by setting $s_{1} \preceq_{\Gamma} s_{2}$ if and only if there is a directed path from $s_{2}$ to $s_{1}$. Note that $\preceq_{\Gamma}$ is a partial order if and only if $\Gamma$ is acyclic. Let $E(S)$ be the set of acyclic directed graphs with vertex set $S$ such that all $\Gamma \in E(S)$ have the following property: If $\rho_{1}$ and $\rho_{2}$ are two distinct directed paths in $\Gamma$ from $x$ to $y$, then $\rho_{1}$ and $\rho_{2}$ have length $\geq 2$. It is easy to check that $(S, \prec) \mapsto \Gamma\left(S, \tau_{\prec}\right)$ and $\Gamma \mapsto \preceq_{\Gamma}$ yields a bijection between the set of partial orders on $S$ and $E(S)$.

Lemma 17.4. $X$ is connected if and only if $\Gamma(X)$ is connected as an undirected graph.

Proof. Assume first that $X$ is connected. Let $x_{0} \in X$ and set

$$
X_{1}:=\left\{x \in X \mid \exists \text { path from } x_{0} \text { to } x \text { in } \Gamma(X)\right\} .
$$

Note that if $y \in \overline{\{x\}}$, then there is an undirected path from $x$ to $y$. Hence, if $x \in X_{1}$, then $\overline{\{x\}} \subseteq X_{1}$. Therefore, $\bigcup_{x \in X_{1}} \overline{\{x\}}=X_{1}$ and $X_{1}$ is closed. On the other hand, if $x \notin X_{1}$, then $\overline{\{x\}} \subseteq X \backslash X_{1}$, hence $X_{1}=\bigcap_{x \notin X_{1}} X \backslash \overline{\{x\}}$ is open. Since $X$ is connected and $X_{1}$ is non-empty, we have $X=X_{1}$.

Now assume that $\Gamma(X)$ is connected as an undirected graph and that $X=X_{1} \sqcup X_{2}$ can be written as a disjoint union of non-empty clopen sets $X_{1}$ and $X_{2}$. Let $x_{i} \in X_{i}$, $i=1,2$ and $\rho$ an undirected path from $x_{1}$ to $x_{2}$. We find neighbouring vertices $y_{1}$ and $y_{2}$ on the path $\rho$ such that $y_{i} \in X_{i} i=1,2$. Wiog gंwe may assume that $y_{2} \in \overline{\left\{y_{1}\right\}}$. Since $X_{1}$ is closed, we have $y_{2} \in \overline{\left\{y_{1}\right\}} \subseteq X_{1}$, which is a contradiction.

\section{$18 \mathfrak{K} \mathfrak{K}(X)$ and Filtrated K-theory}

\section{$18.1 X$-equivariant KK-theory}

As explained in [30] 3.1, there is a version of bivariant K-theory for $C^{*}$-algebras over $X$. Let $A, B \in \in \mathfrak{C}^{*} \mathfrak{s e p}(X)$, a cycle in $\operatorname{KK}(X ; A, B)$ is given by $(E, T)$, where $(E, T)$ is a cycle 
for $\operatorname{KK}(A, B)$, which is $X$-equivariant, that is, $A(U) \cdot E \subseteq E \cdot B(U)$ for all $U \in \mathbb{O}(X)$. There is also a Kasparov product

$$
\mathrm{KK}(X ; A, B) \otimes \mathrm{KK}(X ; B, C) \rightarrow \mathrm{KK}(X ; A, C) .
$$

Thus we may define the category $\mathfrak{K} \mathfrak{K}(X)$ whose objects are separable $C^{*}$-algebras over $X$ and morphisms from $A$ to $B$ are given by $\operatorname{KK}(X ; A, B)$. As shown in [30] 3.2, $\mathfrak{K} \mathfrak{K}(X)$ carries all basic structures we would expect from a bivariant K-theory. In particular, it is additive, has countable coproducts, exterior products, satisfies Bott periodicity and has six term exact sequences for semi-split extensions of $C^{*}$-algebras over $X$. Here, $I \longmapsto A \rightarrow Q$ is an extension of $C^{*}$-algebras over $X$ if $I, A$ and $Q$ are $C^{*}$-algebras over $X$ and $I(U) \longmapsto A(U) \rightarrow Q(U)$ is an extension for all $U \in \mathbb{O}(X)$. It is called semi-split if and only if there is a c.c.p. split section $s$, which is $X$-equivariant in the sense that $s(Q(U)) \subseteq A(U)$ for all $U \in \mathbb{O}(X)$.

$\mathfrak{K} \mathfrak{K}(X)$ carries the structure of a triangulated category ([30] 3.3). The suspension functor is given by the exterior product with $\mathcal{C}_{0}(\mathbb{R})$ and a sequence $S B \rightarrow C \rightarrow A \rightarrow B$ is an exact triangle if and only if it is isomorphic to a mapping cone triangle $S B^{\prime} \rightarrow$ $C_{\phi} \rightarrow A^{\prime} \rightarrow B^{\prime}$ for some $X$-equivariant $*$-homomorphism $\phi: A^{\prime} \rightarrow B^{\prime}$. (Note that the mapping cone has a canonical structure of a $C^{*}$-algebra over $X$ given by $C_{\phi}(U):=$ $C_{\phi_{\mid A^{\prime}(U)}}$. Equivalently, one could define the exact triangles to be those triangles which are isomorphic to the extension triangle of a semi-split extension of $C^{*}$-algebras over $X$ (again, see ([30] 3.3). $\mathfrak{K} \mathfrak{K}(X)$ is a triangulated category with countable coproducts. Following Definition 5.1, we may form $\mathfrak{K} \mathfrak{K}(X)_{*}$. As in the case of $\mathfrak{K}_{\mathfrak{K}}^{G}(8.1)$, it suffices to work with the simpler $\mathbb{Z} / 2$-graded variant, which we will also denote by $\mathfrak{K} \mathfrak{K}(X)_{*}$.

\subsection{Filtrated K-theory}

For a locally closed subset $Y \subseteq X$, one defines a functor

$$
\mathrm{FK}_{Y}^{X}: \mathfrak{K} \mathfrak{K}(X) \rightarrow \mathfrak{A} \mathfrak{b}^{\mathbb{Z} / 2}, \quad \operatorname{FK}_{Y}^{X}(A):=\mathrm{K}_{*}(A(Y)) .
$$

For each $Y \in \mathbb{L} \mathbb{C}(X)$, the functor $\mathrm{FK}_{Y}^{X}$ is stable and homological, that is, it intertwines the suspension on $\mathfrak{K} \mathfrak{K}(X)$ with the translation functor on $\mathfrak{A} \mathfrak{b}^{\mathbb{Z} / 2}$.

Let $\mathcal{N} \mathcal{T}^{X}$ be the $\mathbb{Z} / 2$-graded category whose object set is $\mathbb{L} \mathbb{C}$ and whose morphism space $Y \rightarrow Z$ is $\mathcal{N T}_{*}^{X}(Y, Z)$-the $\mathbb{Z} / 2$-graded Abelian group of all natural transformations $\mathrm{FK}_{Y}^{X} \Rightarrow \mathrm{FK}_{Z}^{X}$. A module over $\mathcal{N} \mathcal{T}^{X}$ is a grading preserving, additive functor $G: \mathcal{N} \mathcal{T}^{X} \rightarrow \mathfrak{A b}^{\mathbb{Z} / 2}$. Let $\mathfrak{M o d}\left(\mathcal{N} \mathcal{T}^{X}\right)$ be the category of $\mathcal{N} \mathcal{T}^{X}$-modules. The morphisms in $\mathfrak{M o d}\left(\mathcal{N} \mathcal{T}^{X}\right)$ are the natural transformations of functors or, equivalently, families of grading preserving group homomorphisms $G_{Y} \rightarrow G_{Y}^{\prime}$ that commute with the actions of $\mathcal{N} \mathcal{T}^{X}$. Let $\mathfrak{M o d}\left(\mathcal{N} \mathcal{T}^{X}\right)_{\text {c }}$ be the full subcategory of countable modules.

Filtrated K-theory is the functor

$$
\mathrm{FK}^{X}=\left(\mathrm{FK}_{Y}^{X}\right)_{Y \in \mathbb{L} \mathbb{C}(X)}: \mathfrak{K} \mathfrak{K}(X) \rightarrow \mathfrak{M o d}(\mathcal{N} \mathcal{T})_{\mathrm{c}}, \quad A \mapsto\left(\mathrm{K}_{*}(A(Y))\right)_{Y \in \mathbb{L} \mathbb{C}(X)} .
$$

We will drop the superscript $X$ in $\mathrm{FK}^{X}$ if there is no danger of confusion. 
If $Y \in \mathbb{L} \mathbb{C}(X)$ is not connected, that is, $Y=Y_{1} \sqcup Y_{2}$ with two disjoint relatively open subsets $Y_{1}, Y_{2} \in \mathbb{O}(Y) \subseteq \mathbb{L} \mathbb{C}(X)$, then any $\mathcal{N} \mathcal{T}$-module has $G_{Y} \cong G_{Y_{1}} \oplus G_{Y_{2}}$. Since $X$ is finite, any locally closed subset is a disjoint union of its connected components. This corresponds to a direct sum decomposition $Y \cong \bigoplus_{j \in \pi_{0}(Y)} Y_{j}$ in $\mathcal{N} \mathcal{T}$. Therefore, we lose no information if we replace $\mathbb{L} \mathbb{C}(X)$ by the subset $\mathbb{L} \mathbb{C}(X)_{c}$ of non-empty, connected, locally closed subsets.

\subsection{The Representability Theorem}

We want to show that filtrated K-theory is a special case of the Hom-like invariants constructed in 5. Recall the representability theorem from [28]:

Theorem 18.1. Let $X$ be a finite topological space. The covariant functors $\mathrm{FK}_{Y}$ for $Y \in \mathbb{L} \mathbb{C}(X)$ are representable, that is, there are objects $\mathcal{R}_{Y} \in \in \mathfrak{K} \mathfrak{K}(X)$ and natural isomorphisms

$$
\mathrm{KK}_{*}\left(X ; \mathcal{R}_{Y}, A\right) \cong \mathrm{FK}_{Y}^{X}(A)=\mathrm{K}_{*}(A(Y))
$$

for all $A \in \in \mathfrak{K} \mathfrak{K}(X), Y \in \mathbb{L} \mathbb{C}(X)$.

For a description of the objects $\mathcal{R}_{Y}$, see [28], 2.1. Let $\mathcal{R}(X)$ be the family $\left(\mathcal{R}_{Y}\right)_{Y \in \mathbb{L} \mathbb{C}(X)}$ in $\mathfrak{K} \mathfrak{K}(X)$. The Yoneda lemma yields a natural isomorphism $\mathcal{N}_{*}^{X}(Y, Z) \cong \mathrm{KK}_{*}\left(X ; \mathcal{R}_{Z}, \mathcal{R}_{Y}\right)$ and an isomorphism of categories

$$
\mathcal{N} \mathcal{T}^{X} \cong\left(\mathfrak{K} \mathfrak{K}(X)_{*}^{\mathcal{R}(X)}\right)^{\text {op }}
$$

Note that we defined $\mathcal{N} \mathcal{T}^{X}$-modules to be covariant functors, whereas $\mathfrak{K} \mathfrak{K}(X)_{*}^{\mathcal{R}(X)}$-modules are contravariant functors. Thus the representability theorem shows that filtrated K-theory is isomorphic to $F_{\mathcal{R}(X)}$, where

$$
F_{\mathcal{R}(X)}: \mathfrak{K} \mathfrak{K}(X) \rightarrow \mathfrak{M o d}\left(\mathfrak{K} \mathfrak{K}(X)_{*}^{\mathcal{R}(X)}\right)_{\mathrm{c}}^{\mathbb{Z}}
$$

denotes the Hom-like invariant associated with the family $\mathcal{R}(X)$.

\subsection{Functoriality}

The canonical functor $\mathfrak{C}^{*} \mathfrak{s e p}(X) \rightarrow \mathfrak{K} \mathfrak{K}(X)$ is the universal split exact $C^{*}$-stable functor ([30], Theorem 3.7). Using this universal property, we may extend the functoriality results for $\mathfrak{C}^{*} \mathfrak{a l g}(X)$ in the space variable to $\mathfrak{K} \mathfrak{K}(X)$ : A continuous map $f: X \rightarrow Y$ induces a functor $f_{*}: \mathfrak{K} \mathfrak{K}(X) \rightarrow \mathfrak{K} \mathfrak{K}(Y)$. In particular, this yields an extension functor $i_{X}^{Y}$ for a subspace $X \subseteq Y$. For $Y \in \mathbb{L} \mathbb{C}(X)$, the restriction functor descends to a functor $r_{X}^{Y}: \mathfrak{K} \mathfrak{K}(X) \rightarrow \mathfrak{K} \mathfrak{K}(Y)$.

Our next aim is to construct an algebraic variant of $f_{*}$, i.e. a functor $f_{*}: \mathfrak{M o d}\left(\mathcal{N} \mathcal{T}^{X}\right)_{\mathrm{c}} \rightarrow$ $\mathfrak{M o d}\left(\mathcal{N} \mathcal{T}^{Y}\right)_{\mathrm{c}}$ such that

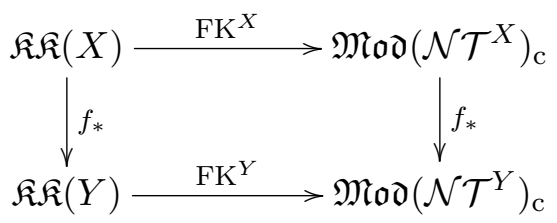


commutes. Let us do this by first constructing a functor $f^{*}: \mathcal{N} \mathcal{T}^{Y} \rightarrow \mathcal{N} \mathcal{T}^{X}$ : For $Z \in \in \mathcal{N} \mathcal{T}^{Y}=\mathbb{L} \mathbb{C}(Y)$, set $f^{*}(Z)=f^{-1}(Z)$. A morphism $\tau \in \mathcal{N} \mathcal{T}^{Y}\left(Z, Z^{\prime}\right)$ is a natural transformation $\tau$ : $\mathrm{FK}_{Z}^{Y} \rightarrow \mathrm{FK}_{Z^{\prime}}^{Y}$, i.e. a collection $\left\{\tau_{A}\right\}_{A \in \in \mathfrak{K} \mathfrak{K}^{Y}}$ of morphisms of abelian groups

$$
\tau_{A}: \mathrm{FK}_{Z}^{Y}(A)=K_{*}(A(Z)) \rightarrow K_{*}\left(A\left(Z^{\prime}\right)\right)=\mathrm{FK}_{Z^{\prime}}^{Y}(A),
$$

which is natural with respect to morphisms in $\mathfrak{C}^{*} \mathfrak{a l g}(Y)$. Since for $B \in \in \mathfrak{K} \mathfrak{K}(X)$ and $Z \in \mathbb{L} \mathbb{C}(Y)$, we have

$$
\mathrm{FK}_{Z}^{Y}\left(f_{*} B\right)=K_{*}\left(B\left(f^{-1}(Z)\right)\right)=\mathrm{FK}_{f^{-1}(Z)}^{X}(B),
$$

$\tau_{f_{*} B}$ is also a morphism from $\mathrm{FK}_{f^{-1}(Z)}^{X}(B)$ to $\mathrm{FK}_{f^{-1}\left(Z^{\prime}\right)}^{X}(B)$ and it makes sense to define

$$
f^{*}(\tau):=\left\{\tau_{f_{*} B}\right\}_{B \in \in \mathfrak{K}_{X}} .
$$

Hence we have constructed an additive grading preserving functor

$$
f^{*}: \mathcal{N} \mathcal{T}^{Y} \rightarrow \mathcal{N} \mathcal{T}^{X}
$$

This gives rise to an additive, grading preserving functor

$$
f_{*}: \mathfrak{M o d}\left(\mathcal{N} \mathcal{T}^{X}\right)_{\mathrm{c}} \rightarrow \mathfrak{M o d}\left(\mathcal{N} \mathcal{T}^{Y}\right)_{\mathrm{c}}, \quad f_{*}(M):=M \circ f^{*} .
$$

Lemma 18.2. Let $X, Y, f$ and $f_{*}$ be as above, then the diagram

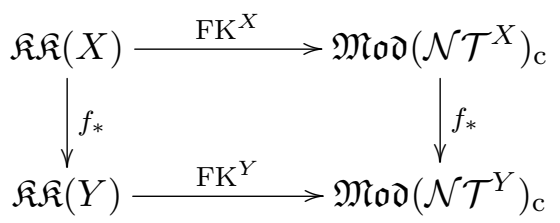

commutes.

Proof. Recall that there is a canonical functor $\operatorname{KK}(X): \mathfrak{C}^{*} \mathfrak{a l g}(X) \rightarrow \mathfrak{K} \mathfrak{K}(X)$. By the universal property of $\operatorname{KK}(X)$ ([30], Theorem 3.7.), we see that it suffices to check that

$$
f_{*} \circ \mathrm{FK}^{X} \circ \mathrm{KK}(X)=\mathrm{FK}^{Y} \circ f_{*} \circ \mathrm{KK}(X) .
$$

On objects, there is no difference anyway: Let $A \in \in \mathfrak{K} \mathfrak{K}(X)$ and $Z \in \mathbb{L} \mathbb{C}(Y)$, then

$$
f_{*} \circ \mathrm{FK}^{X}(A)(Z)=\mathrm{K}_{*}\left(A\left(f^{-1}(Z)\right)=\mathrm{FK}^{Y} \circ f_{*}(A)(Z) .\right.
$$

Let $\phi: A \rightarrow B$ be a morphism of $C^{*}$-algebras over $X$ and $Z \in \mathbb{L} \mathbb{C}(Y)$. Passing to subquotients, $\phi$ induces $*$-homomorphisms $\phi\left(Z^{\prime}\right): A\left(Z^{\prime}\right) \rightarrow B\left(Z^{\prime}\right)$ for all $Z^{\prime} \in \mathbb{L} \mathbb{C}(X)$. $f_{*}(\phi): f_{*}(A) \rightarrow f_{*}(B)$ is a morphism of $C^{*}$-algebras over $Y$, which is given by $\phi$ as a $*$-homomorphism from $A$ to $B$ if we forget the structure over $X$ (or $Y$ ). Note that $f_{*}(\phi)(Z)=\phi\left(f^{-1}(Z)\right)$ as a $*$-homomorphism. Now the equalities

$$
f_{*} \circ \mathrm{FK}^{X} \circ \mathrm{KK}(X)(\phi)(Z)=f_{*} \circ \mathrm{FK}^{X}([\phi])(Z)=\mathrm{FK}^{X}([\phi])\left(f^{-1}(Z)\right)=\mathrm{K}_{*}\left(\phi\left(f^{-1}(Z)\right)\right)
$$

and

$$
\mathrm{FK}^{Y} \circ f_{*} \circ \mathrm{KK}(X)(\phi)(Z)=\mathrm{FK}^{Y}\left(\left[f_{*}(\phi)\right]\right)(Z)=\mathrm{K}_{*}\left(f_{*}(\phi)(Z)\right)=\mathrm{K}_{*}\left(\phi\left(f^{-1}(Z)\right)\right)
$$

yield the desired result. 


\section{The Bootstrap Class and the UCT Criterion}

\subsection{Comparing Bootstrap Classes}

In [30], R. Meyer and R. Nest define the bootstrap class $\mathcal{B}(X)$ to be the localizing subcategory of $\mathfrak{K} \mathfrak{K}(X)$ generated by the objects $i_{x} \mathbb{C}$ for all $x \in X$. That is, it is the smallest class of objects containing these generators that is closed under suspensions, $\mathrm{KK}(X)$-equivalence, semi-split extensions, and countable direct sums.

Our general framework would tell us to define the bootstrap class as the localizing subcategory generated by the $\mathcal{R}_{Y}, Y \in \mathbb{L} \mathbb{C}(X)$. A natural question to ask is whether both definition agree and in fact, they do:

Lemma 19.1. $\mathcal{B}(X)$ is equal to the localizing subcategory generated by $\mathcal{R}_{Y}, Y \in \mathbb{L} \mathbb{C}(X)$.

Proof. Let $\mathcal{B}(X)^{\prime}$ denote the localizing subcategory generated by $\mathcal{R}_{Y}, Y \in \mathbb{L} \mathbb{C}(X) . X$ is finite, therefore, every $x \in X$ has a minimal open neighbourhood $U_{x}$. In [28], proof of Theorem 2.5, it is shown that $i_{x} \mathbb{C}$ is isomorphic to $\mathcal{R}_{U_{x}}$ in $\mathfrak{K} \mathfrak{K}(X)$ for all $x \in X$. This shows that $\mathcal{B}(X)$ is contained in $\mathcal{B}(X)^{\prime}$. For the other direction, let Good be the set of locally closed subsets $Y$ such that $\mathcal{R}_{Y} \in \in \mathcal{B}(X)$. Let $Y \in \mathbb{L} \mathbb{C}(X), U \in \mathbb{O}(Y)$. The existence of the exact triangle

$$
S \mathcal{R}_{U} \rightarrow \mathcal{R}_{Y \backslash U} \rightarrow \mathcal{R}_{Y} \rightarrow \mathcal{R}_{U}
$$

shows that $Y, U$ and $Y \backslash U$ are in Good if two of them are. Since $(U \cup V) \backslash U=V \backslash(U \cap V)$ we have

$$
U, V \in \mathbb{O}(X), U, V, U \cap V \in \operatorname{Good} \Rightarrow U \cup V \in \operatorname{Good} .
$$

Induction over the cardinality of $U \in \mathbb{O}(X)$ shows that $\mathbb{O}(X) \subseteq$ Good. Since every locally closed set is the difference of two open sets, this implies the claim.

Corollary 19.2. Let $f: X \rightarrow Y$ be continuous and $A \in \in \mathcal{B}(X)$, then $f_{*}(A) \in \in \mathcal{B}(Y)$.

Proof. $f_{*}\left(\iota_{x} \mathbb{C}\right)=\iota_{f(x)} \mathbb{C}$, therefore, generators of $\mathcal{B}(X)$ are sent to generators of $\mathcal{B}(Y)$. Since $f_{*}: \mathfrak{K} \mathfrak{K}(X) \rightarrow \mathfrak{K} \mathfrak{K}(Y)$ is triangulated, this shows the claim.

\subsection{The UCT Criterion and Embedding Results}

Our general UCT criterion (Theorem 7.1) and the representability theorem (Theorem 18.1) show what is actually needed to obtain a UCT short exact sequence, which computes $\mathrm{KK}(X, \ldots, \ldots)$ in terms of filtrated $\mathrm{K}$-theory:

Theorem 19.3. Let $A, B \in \in \mathfrak{K} \mathfrak{K}(X)$. Suppose that $\mathrm{FK}^{X}(A) \in \in \mathfrak{M o d}\left(\mathcal{N} \mathcal{T}^{X}\right)_{\mathrm{c}}$ has a projective resolution of length 1 and that $A \in \in \mathcal{B}(X)$. Then there are natural short exact sequences

$$
\operatorname{Ext}_{\mathcal{N} \mathcal{T}^{X}}^{1}\left(\mathrm{FK}^{X}(A)[j+1], \operatorname{FK}(B)\right) \longmapsto \operatorname{KK}_{j}(X ; A, B) \rightarrow \operatorname{Hom}_{\mathcal{N} \mathcal{T}^{X}}\left(\mathrm{FK}^{X}(A)[j], \operatorname{FK}^{X}(B)\right)
$$

for $j \in \mathbb{Z} / 2$, where $\operatorname{Hom}_{\mathcal{N} \mathcal{T}^{X}}$ and $\operatorname{Ext}_{\mathcal{N T}^{X}}^{1}$ denote the morphism and extension groups in the Abelian category $\mathfrak{M o d}\left(\mathcal{N} \mathcal{T}^{X}\right)_{\mathrm{c}}$ and $[j]$ and $[j+1]$ denote degree shifts. 
Since we are asking which spaces have the property of having a UCT s.e.s. for filtrated K-theory, it makes sense to view the crucial assumption in the Theorem above as a property of the space $X$.

Definition 19.4. Let $X$ be a finite $T_{0}$ space. We say that $U C T(X)$ holds if for all $A \in \in \mathfrak{K} \mathfrak{K}(X), \operatorname{FK}^{X}(A) \in \in \mathfrak{M} \mathfrak{o d}\left(\mathcal{N} \mathcal{T}^{X}\right)_{\mathrm{c}}$ has a projective resolution of length 1 .

As stated above we may restrict attention to connected spaces:

Lemma 19.5. Let $X$ be a finite $T_{0}$ space, which is a disjoint union of spaces $X_{1}, \ldots X_{n}$, then $U C T(X)$ holds if and only if $U C T\left(X_{i}\right)$ holds for $i=1, \ldots, n$.

Let us also mention an important conclusion, which can be drawn from the existence of a UCT short exact sequence (this is Corollary 4.9 of [28]):

Corollary 19.6. Let $A, B \in \in \mathcal{B}(X)$ and suppose that both $\mathrm{FK}(A)$ and $\mathrm{FK}(B)$ have projective resolutions of length 1 in $\mathfrak{M o d}(\mathcal{N} \mathcal{T})_{\mathrm{c}}$. Then any morphism $\mathrm{FK}(A) \rightarrow \mathrm{FK}(B)$ in $\mathfrak{M o d}(\mathcal{N T})_{\mathrm{c}}$ lifts to an element in $\mathrm{KK}_{0}(X ; A, B)$, and an isomorphism $\mathrm{FK}(A) \cong \operatorname{FK}(B)$ lifts to an isomorphism in $\mathcal{B}(X)$.

The possibility of lifting isomorphisms in filtrated K-theory to isomorphisms in $\mathfrak{K} \mathfrak{K}(X)$ is one of the main reasons why one is interested in a UCT short exact sequence. On the other hand, the impossibility of lifting isomorphisms in $\mathrm{FK}^{X}$ is an obstruction to the existence of a UCT short exact sequence.

Definition 19.7. Let $X$ be a finite $T_{0}$ space. We say that $\neg U C T(X)$ holds if there are $A, B \in \in \mathcal{B}(X)$ such that $A \nsubseteq B$ in $\mathfrak{K} \mathfrak{K}(X)$ and $\mathrm{FK}^{X}(A) \cong \mathrm{FK}^{X}(B)$ in $\mathfrak{M} \mathfrak{o d}\left(\mathcal{N} \mathcal{T}^{X}\right)_{\mathrm{c}}$.

It is clear that there is no finite $T_{0}$ space such that both $U C T(X)$ and $\neg U C T(X)$ hold. Moreover, as suggested by notation, we will show that for every such $X$ either $U C T(X)$ or $\neg U C T(X)$ holds.

The next proposition tells us, roughly, that if $X$ has a subspace for which there is no $\mathrm{UCT}$, then there cannot exist a UCT for $X$ as well.

Proposition 19.8. Let $X$ be a space, $Y \in \mathbb{L} \mathbb{C}(X)$ such that $\neg U C T(Y)$ holds, then $\neg U C T(X)$ holds as well.

Let $X$ and $Y$ be topological spaces, $f: X \rightarrow Y$ and $g: Y \rightarrow X$ continuous with $f \circ g=$ $\operatorname{id}_{Y}$. Assume that $\neg U C T(Y)$ holds, then $\neg U C T(X)$ holds as well.

Proof. By assumption, there are $A, B \in \in \mathfrak{K} \mathfrak{K}(Y)$ such that $A \not B$ in $\mathfrak{K} \mathfrak{K}(Y)$ and $\mathrm{FK}^{Y}(A) \cong \mathrm{FK}^{Y}(B)$. As already noted above, we have $r_{X}^{Y} \circ i_{Y}^{X}=\operatorname{id}_{Y}$ (see also [30] Lemma 2.20 (c)), therefore, $i_{Y}^{X}(A) \nsucceq i_{Y}^{X}(B)$ in $\mathfrak{K}_{\mathfrak{K}}$, furthermore $i_{Y}^{X}(A), i_{Y}^{X}(B) \in \in \mathcal{B}(X)$ by Corollary 19.2. Recall that $i_{Y}^{X}$ is just $\iota_{*}$ for the embedding $\iota: Y \hookrightarrow X$. Hence

$$
\mathrm{FK}^{X}\left(i_{Y}^{X}(A)\right)=\iota_{*}\left(\mathrm{FK}^{Y}(A)\right) \cong \iota_{*}\left(\mathrm{FK}^{Y}(B)\right)=\mathrm{FK}^{X}\left(i_{Y}^{X}(B)\right)
$$

by Lemma 18.2. This shows the first statement.

By assumption, there are $A, B \in \in \mathfrak{K} \mathfrak{K}(Y)$ such that $A \nsubseteq B$ in $\mathfrak{K} \mathfrak{K}(Y)$ and $\operatorname{FK}^{Y}(A) \cong$ $\mathrm{FK}^{Y}(B)$. Since $f_{*} \circ g_{*}=\operatorname{id}_{\mathfrak{K} \mathfrak{K}(Y)}$ we have that $g_{*}(A) \varsubsetneqq g_{*}(B)$, furthermore $g_{*}(A), g_{*}(B) \in \in$ $\mathcal{B}(X)$ by Corollary 19.2. $g_{*} \circ \mathrm{FK}^{Y}=\mathrm{FK}^{X} \circ g_{*}$ implies $\mathrm{FK}^{X}\left(g_{*}(A)\right) \cong \mathrm{FK}^{X}\left(g_{*}(B)\right)$ (again by Lemma 18.2). This shows the second statement. 


\section{Positive Results and Counterexamples}

In this section we introduce spaces of type $A$ and some spaces, which will serve as generic counterexamples for a classification of $C^{*}$-algebras over finite spaces via filtrated K-theory.

Definition 20.1. Let $X$ be a finite $T_{0}$ space. We say that $X$ is of type $A$ (for accordion) if exactly two vertices in $\Gamma(X)$ have degree 1 and all other vertices have degree 2 .

The following picture shows the graph associated with a space of type $A$ :
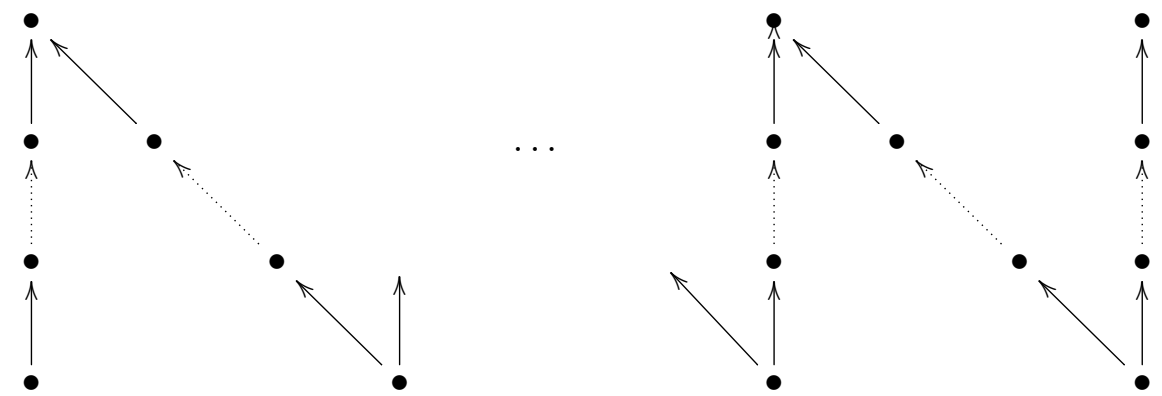

Theorem 20.2. Let $X$ be a finite $T_{0}$ space of type $A$, then $U C T(X)$ holds.

Proof. This is shown in [4].

Following R. Bentmann, we will now introduce some spaces, for which there is no UCT short exact sequence for filtrated K-theory. If $X$ is a space, let $X^{\text {op }}$ denote its dual space, i.e. $X^{\mathrm{op}}=X$ as a set and the open sets in $X^{\mathrm{op}}$ are exactly the closed sets in $X$.

Definition 20.3. Let us define the following spaces:

1. $X_{1}=\{1,2,3,4\}, \tau_{X_{1}}=\left\{\emptyset, X_{1},\{1\},\{2\},\{3\}\right\}$;

2. $X_{2}=X_{1}^{\mathrm{op}}$

3. $X_{3}=\{1,2,3,4\}, \tau_{X_{3}}=\left\{\emptyset, X_{3},\{1\},\{2\},\{1,2,3\}\right\}$;

4. $X_{4}=X_{3}^{\mathrm{op}}$;

5. $S=\{1,2,3,4\}, \tau_{S}=\{\emptyset, S,\{1\},\{1,2\},\{1,3\},\{1,2,3\}\}$;

6. $C_{n}=\mathbf{C}(n) \times\{a, b\}$, a basis of $\tau_{C_{n}}$ is given by $\{(k, a),(k, b),(k+[1], a)\}_{k \in \mathbf{C}(n)}$ for $n \geq 2$.

The associated directed graphs look as follows:

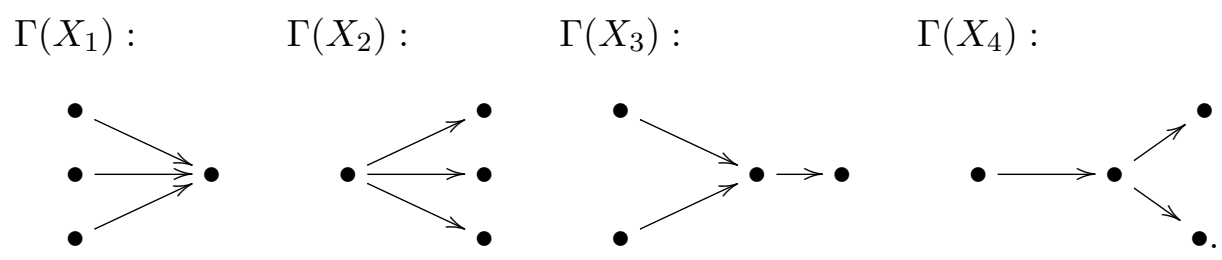




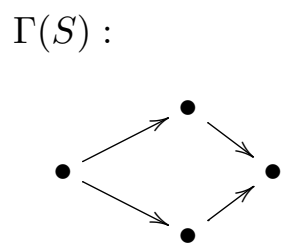

$\Gamma\left(C_{n}\right):$

Theorem 20.4. Let $X$ be a space of the form $X_{1}, X_{2}, X_{3}, X_{4}, S$ or $C_{n}$ for $n \geq 2$. Then $\neg U C T(X)$ holds.

Proof. This is shown in [5] for $X_{1}$ and $X_{3}$ and in [4] for the rest.

\section{The Complete Description}

We already know that if $X$ is of type $A$, then $U C T(X)$ holds. The aim of this section is to prove the converse. We want to show that if $X$ is not of type $A$, then we can "embed" one of the counterexamples from Section 20 in $X$. Knowing that $\neg U C T$ holds for the counterexample, we will use the embedding result from Section 19 to conclude that $\neg U C T(X)$ holds.

Definition 21.1. A topological subspace $X^{\prime}$ of a finite $T_{0}$ space $X$ is tight if

$$
y \rightarrow x \text { in } X^{\prime} \Leftrightarrow y \rightarrow x \text { in } X .
$$

If $X^{\prime}$ is a topological subspace of $X$ then $X^{\prime}$ is tight in $X$ if and only if every arrow in $\Gamma\left(X^{\prime}\right)$ is also an arrow in $\Gamma(X)$. If $Y$ is another finite $T_{0}$ space such that there exists an embedding $\Gamma(Y) \hookrightarrow \Gamma(X)$ as directed graphs, then $Y$ may be viewed as a tight subspace of $X$.

Lemma 21.2. Let $X$ be a finite $T_{0}$ space such that $\Gamma(X)$ contains either $\Gamma\left(X_{1}\right)$ or $\Gamma\left(X_{2}\right)$ as a subgraph, then $\neg U C T(X)$ holds.

Proof. $\Gamma\left(X_{1}\right) \subseteq \Gamma(X)$ allows us to view $X_{1}$ as a tight subspace of $X$. Let $y \in L C\left(X_{1}\right)$, then there are $x_{1}, x_{2} \in X_{1}$ such that $x_{1} \preceq y \preceq x_{2}$. Without loss of generality, we may assume that $x_{1}=1$ and $x_{2}=4$. Since $1 \rightarrow 4$ we have $y=1$ or $y=4$ by Lemma 17.3. Therefore, $X_{1}$ is locally closed in $X$. Similarly, we see that $X_{2}$ is locally closed in $X$ if $\Gamma\left(X_{2}\right) \subseteq \Gamma(X)$. Therefore, $\neg U C T(X)$ holds by Theorem 20.4 and Proposition 19.8 (b).

Proposition 21.3. Let $X$ be a space such that $\Gamma(X)$ contains $\Gamma\left(X_{3}\right)$ as a subgraph. Set

$$
\pi_{3}: L C\left(X_{3}\right) \rightarrow X_{3}, \quad \pi_{3}(x)= \begin{cases}x & \text { if } x \in X_{3} \\ 3 & \text { otherwise }\end{cases}
$$

Then $\pi_{3}$ is continuous. 
Proof. Let us first show the following claim:

Claim \#1: If $x \in L C\left(X_{3}\right) \backslash X_{3}$, then $x \succ 4, x \npreceq 3, x \nsucceq 3, x \nsucceq 1, x \nsucceq 2$.

Let $x \in L C\left(X_{3}\right) \backslash X_{3}$, then there are $x_{1}, x_{2} \in X_{3}$ such that $x_{1} \prec x \prec x_{2}$. Since $1 \rightarrow 3,2 \rightarrow 3,3 \rightarrow 4$, Lemma 17.3 shows that $x_{1}=4$ and $x_{2} \in\{1,2\}$, w.l.o.g. we may assume that $x_{2}=1$. This implies that $x \nsucceq 1$ and $x \succ 4$. Assume $x \succeq 2$, then $1 \succ x \succ 2 \succ 3$ and since $x \notin X_{3}$, by Lemma 17.3, this is a contradiction to $1 \rightarrow 3$. By the same argument, $x \succeq 3$ leads to a contradiction. Assume $x \preceq 3$, then $4 \prec x \prec 3$ this is a contradiction to $3 \rightarrow 4$. This shows the claim.

To show that $\pi_{3}$ is continuous we have to check that it is monotone. Let $x, y \in L C\left(X_{3}\right)$, if $x, y \in X_{3}$ then $x \preceq y$ clearly implies $\pi_{3}(x) \preceq \pi_{3}(y)$. If $x, y \in L C\left(X_{3}\right) \backslash X_{3}$ then $\pi_{3}(x)=3=\pi_{3}(y)$. If $x \in L C\left(X_{3}\right) \backslash X_{3}, y \in X_{3}$ and $y \prec x$, then $y=4$ by Claim \#1. Therefore, $\pi_{3}(4)=4 \prec 3=\pi_{3}(x)$. If $y \in X_{3}, x \in L C\left(X_{3}\right) \backslash X_{3}$ and $y \succ x$, then either $y=1$ or $y=2$ by Claim \# 1 and in both cases $\pi_{3}(y)=y \succ 3=\pi_{3}(x)$. This shows that $\pi_{3}$ is continuous.

Proposition 21.4. Let $X$ be a space such that $\Gamma(X)$ contains $\Gamma\left(X_{4}\right)$ as a subgraph. Set

$$
\pi_{4}: L C\left(X_{4}\right) \rightarrow X_{4}, \quad \pi_{4}(x)= \begin{cases}x & \text { if } x \in X_{4} \\ 3 & \text { otherwise }\end{cases}
$$

Then $\pi_{4}$ is continuous.

Proof. This is proven completely analogously to Proposition 21.3, just switch $\prec$ and $\succ$ in the proof.

Corollary 21.5. Let $X$ be a space such that $\Gamma(X)$ contains either $\Gamma\left(X_{3}\right)$ or $\Gamma\left(X_{4}\right)$ as a subgraph, then $\neg U C T(X)$ holds.

Proof. Assume $\Gamma\left(X_{3}\right) \subseteq \Gamma(X)$ and let $Y=L C\left(X_{3}\right)$. There is an inclusion $\iota_{3}: X_{3} \hookrightarrow$ $L C\left(X_{3}\right)$ and $\pi_{3}: L C\left(X_{3}\right) \rightarrow X_{3}$ from Proposition 21.3. By construction, $\pi_{3} \circ \iota_{3}=\mathrm{id}_{X_{3}}$. This shows that $\neg U C T\left(L C\left(X_{3}\right)\right)$ holds by Proposition $19.8(2)$, hence $\neg U C T(X)$ holds by Proposition 19.8 (1). The same arguments using $\iota_{4}: X_{4} \hookrightarrow L C\left(X_{4}\right)$ and $\pi_{4}$ from Proposition 21.4 show the corresponding statement for $X_{4}$.

Corollary 21.6. Let $X$ be a finite $T_{0}$ space such that $\Gamma(X)$ has a vertex of degree $\geq 3$, then $\neg U C T(X)$ holds.

Proof. $\Gamma(X)$ must contain either $\Gamma\left(X_{1}\right), \Gamma\left(X_{2}\right), \Gamma\left(X_{3}\right)$ or $\Gamma\left(X_{4}\right)$ as a subgraph.

Proposition 21.7. Let $X$ be such that every vertex of $\Gamma(X)$ has degree 2. Then $\neg U C T(X)$ holds.

Proof. The assumption means that $\Gamma(X)$ as an undirected graph consists of a circle. Recall the definition of the oriented degree $d_{o}$ from Section 17.4. By assumption, we have

$$
d_{o}(x) \in\{-2,0,2\} \forall x \in X \text { and } \sum_{x \in X} d_{o}(x)=0,
$$


This means that there are as many vertices with oriented degree 2 as vertices with oriented degree -2 . Let $n$ be the number of vertices with oriented degree 2 . Since $\Gamma(X)$ cannot be a directed circle, $n$ is at least 1 .

Case (a): $n=1$. There is exactly one vertex $a$ with oriented degree 2 , one vertex $b$ with oriented degree -2 and two directed paths $\rho=\left(v_{i}\right)_{i=0, \ldots, n}$ and $\sigma=\left(w_{i}\right)_{i=0, \ldots, m}$ from $a$ to $b$ such that

$$
\rho \cap \sigma=\{a, b\}, \quad \rho \cup \sigma=X .
$$

Define maps $f: X \rightarrow S$ and $g: S \rightarrow X$ via

$$
f(x)=\left\{\begin{array}{ll}
1 & \text { if } x=a \\
2 & \text { if } x=v_{i} \text { for } i=1, \ldots, n-1 \\
3 & \text { if } x=w_{i} \text { for } i=1, \ldots, m-1 \\
4 & \text { if } x=b
\end{array} \text { and } g(s)=\left\{\begin{array}{ll}
a & \text { if } s=1 \\
v_{1} & \text { if } s=2 \\
w_{1} & \text { if } s=3 \\
b & \text { if } s=4
\end{array},\right.\right.
$$

$f$ and $g$ are continuous since they are monotone. It is clear that $f \circ g=\operatorname{id}_{S}$, therefore, $\neg U C T(X)$ holds by Theorem 20.4 and Proposition 19.8 (b).

Case (b): $n>1$. We will basically proceed as in case (a), only notation becomes slightly more complicated. Let $\mathbf{C}(n)$ denote the cyclic group of order $n$. Ordering the vertices of oriented degree 2 and -2 clockwise, we obtain sequences $\left(a_{k}\right)_{k \in \mathbf{C}(n)}$ and $\left(b_{k}\right)_{k \in \mathbf{C}(n)}$ in $X$ such that $d_{o}\left(a_{k}\right)=2$ and $d_{o}\left(b_{k}\right)=-2$ for all $k \in \mathbf{C}(n)$. Analogously to case (a), there is a sequence of directed paths $\left(\rho^{k}=\left(v_{i}^{k}\right)_{i=1, \ldots, n_{k}}\right)_{k \in \mathbf{C}(n)}$, where $\rho^{k}$ is a path from $a_{k}$ to $b_{k}$ and a sequence of directed paths $\left(\sigma^{k}=\left(w_{i}^{k}\right)_{i=1, \ldots, m_{k}}\right)_{k \in \mathbf{C}(n)}$, where $\sigma^{k}$ is a path from $a_{k}$ to $b_{k-[1]}$ such that

$$
\rho^{k} \cap \rho^{l}=\sigma^{k} \cap \sigma^{l}=\varnothing \text { if } k \neq l, \quad \rho^{k} \cap \sigma^{l}= \begin{cases}a_{k} & \text { if } k=l \\ b_{k} & \text { if } k=l-[1] \\ \varnothing & \text { otherwise. }\end{cases}
$$

and

$$
\bigcup_{k \in \mathbf{C}(k)} \rho^{k} \cup \bigcup_{k \in \mathbf{C}(k)} \sigma^{k}=X
$$

Define maps $f: X \rightarrow C_{n}$ and $g: C_{n} \rightarrow X$ via

$$
f(x)=\left\{\begin{array}{ll}
(k, a) & \text { if } x=a_{k}, \\
(k, b) & \text { if } x=v_{i}^{k} \text { for } i=1, \ldots, n_{k}, \\
(k-[1], b) & \text { if } x=w_{i}^{k} \text { for } i=1, \ldots, m_{k}-1,
\end{array} \text { and } g((k, y))= \begin{cases}a_{k} & \text { if } y=a, \\
b_{k} & \text { if } y=b .\end{cases}\right.
$$

$f$ and $g$ are continuous since they are monotone. It is clear that $f \circ g=\mathrm{id}_{C_{n}}$, therefore, $\neg U C T(X)$ holds by Theorem 20.4 and Proposition 19.8 (b).

Theorem 21.8. Let $X$ be a finite $T_{0}$ space. Then $U C T(X)$ holds if and only if $X$ is a disjoint union of spaces of type $A$. 
Proof. That $U C T(X)$ holds if $X$ is a disjoint union of spaces of type $A$ follows from Theorem 20.2 and Lemma 19.5. Now let $X$ be a space such that $U C T(X)$ holds, by Lemma 19.5, it suffices to show that $X$ is of type $A$ under the assumption that $X$ is connected (and hence, by Lemma 17.4, that $\Gamma(X)$ is connected as an undirected graph). By Corollary 21.6, all vertices $x$ of $\Gamma(X)$ have degree less than 3. By the last remark and Proposition 21.7, there is at least one vertex of degree less than 2. Since $\Gamma(X)$ is connected as an undirected graph and finite, there are exactly two vertices of degree 1 and all other vertices have degree 2 , therefore, $X$ is of type $A$ as claimed. 


\section{Appendix}

\section{Facts about Cyclotomic Polynomials}

Definition 22.1. For $n \in \mathbb{N}$, let $\Phi_{n}$ denote the $n$th cyclotomic polynomial, i.e. the monic polynomial which is given by

$$
\Phi_{n}(t)=\prod_{\omega n \text {th primitive root of unity }}(t-\omega)
$$

Theorem 22.2. Let $n$ be an integer, then

$(1)$

$$
t^{n}-1=\prod_{m \mid n} \Phi_{m}(t)
$$

(2) $\Phi_{n}(t) \in \mathbb{Z}[t]$;

(3) We have

$$
\operatorname{deg} \Phi_{n}=\operatorname{dim}_{\mathbb{Z}}\left(\mathbb{Z}\left[\theta_{n}\right]\right)=\phi(n),
$$

where $\phi$ denotes Euler's totient function.

(4) Let $p$ be a prime number and $n \in \mathbb{N}$, then

$$
\Phi_{p^{n}}(t)=\sum_{i=0}^{p-1} t^{p^{n-1}}
$$

Proof. See [36], Section 3.3.

Lemma 22.3. Let $\theta_{n}$ denote a primitive $n$th root of unity, then there is an isomorphism of rings

$$
\mathbb{Z}\left[\theta_{n}\right] \cong \mathbb{Z}[t] /\left\langle\Phi_{n}\right\rangle
$$

Proof. [18] p. 172.

The relevance of cyclotomic polynomials in our context is that they allow for a decomposition of the of $\mathbb{Z}\left[n^{-1}\right] \mathbf{C}(n)$ into a direct sum of Dedekind domains. Before proving this, let us collect a few basic definitions and facts, which we state separately since they will be of use later on. 
Appendix

Definition 22.4. Let $n \in \mathbb{N}$. For $m \mid n$, define

$$
\psi_{n, m}(t):=\frac{1}{n} \cdot t \cdot \frac{\mathrm{d}}{\mathrm{d} t} \Phi_{m}(t) \cdot \prod_{m^{\prime} \mid n, m^{\prime} \neq m} \Phi_{m^{\prime}}(t) \in \mathbb{Z}\left[n^{-1}\right][t] .
$$

We will sometimes state polynomial identities in quotient rings of $\mathbb{Z}\left[n^{-1}\right][t]$ such as $\mathbb{Z}\left[n^{-1}\right][t] /\left\langle t^{n}-1\right\rangle$. If $q(t), p(t) \in \mathbb{Z}\left[n^{-1}\right][t]$ such that their residual classes are equal in a quotient ring $R$, we will say $p(t)=q(t)$ in $R$ instead of talking about the residual classes.

Lemma 22.5. Let $n \in \mathbb{N}$. Then $\sum_{m \mid n} \psi_{n, m}(t)=1$ in $\mathbb{Z}\left[n^{-1}\right][t] /\left\langle t^{n}-1\right\rangle$.

Proof. Differentiate $t^{n}-1=\prod_{m \mid n} \Phi_{m}(t)$ and multiply by $\frac{1}{n} \cdot t$.

Lemma 22.6. Let $n \in \mathbb{N}$ and $m, k \mid n$. Then

$$
\psi_{n, m} \cdot \psi_{n, k}=\delta_{k, m} \psi_{n, m} \text { in } \mathbb{Z}\left[n^{-1}\right][t] /\left\langle t^{n}-1\right\rangle
$$

Proof. Let $k \neq m$. By Theorem $22.2(1), t^{n}-1$ divides $\psi_{n, m}(t) \cdot \psi_{n, k}(t)$ if $k \neq m$. Hence $\psi_{n, m}(t) \cdot \psi_{n, k}(t)=0$ in $\mathbb{Z}[t] t /\left\langle t^{n}-1\right\rangle$ in this case. If $k=m$, multiply $\sum_{m \mid n} \psi_{n, m}(t)=1$ with $\psi_{n, k}(t)$ to get $\psi_{n, m}^{2}=\psi_{n, m}$.

For a prime $p$, let us abbreviate $\psi_{p^{k}, p^{u}}$ by $\Psi_{k, u}$.

Lemma 22.7. Let $0 \leq k, u \leq l$, then

$$
\Psi_{l, u}=\left\{\begin{array}{ll}
\Psi_{k, u} & \text { if } u \leq k \\
0 & \text { otherwise }
\end{array} \quad \text { in } \mathbb{Z}\left[p^{-1}\right][t] /\left\langle t^{p^{k}}-1\right\rangle .\right.
$$

Proof. The explicit description of $\phi_{p^{u}}$ in Theorem 22.2 (4) shows that $\Phi_{p^{u}}(t)=p$ in $\mathbb{Z}\left[p^{-1}\right][t] /\left\langle t^{p^{k}}-1\right\rangle$ if $u>k$. Now calculate

$$
\begin{aligned}
\Psi_{l, u}(t) & =p^{-l} \cdot t \cdot \frac{\mathrm{d}}{\mathrm{d} t} \Phi_{p^{u}}(t) \cdot \prod_{0 \leq u^{\prime} \leq l, u^{\prime} \neq u} \Phi_{p^{u^{\prime}}}(t) \\
& = \begin{cases}p^{-l} \cdot t \cdot \frac{\mathrm{d}}{\mathrm{d} t} \Phi_{p^{u}}(t) \prod_{0 \leq u^{\prime} \leq k, u^{\prime} \neq u} \Phi_{p^{u^{\prime}}}(t) \prod_{k+1 \leq u^{\prime} \leq l} \Phi_{p^{u^{\prime}}}(t) & \text { if } u \leq k \\
p^{-l} \cdot t \cdot \frac{\mathrm{d}}{\mathrm{d} t} \Phi_{p^{u}}(t) \prod_{0 \leq u^{\prime} \leq k} \Phi_{p^{u^{\prime}}}(t) \prod_{k+1 \leq u^{\prime} \leq l, u^{\prime} \neq u} \Phi_{p^{u^{\prime}}}(t) & \text { if } u>k\end{cases} \\
& = \begin{cases}p^{-l} \cdot t \cdot \frac{\mathrm{d}}{\mathrm{d} t} \Phi_{p^{u}}(t) \prod_{0 \leq u^{\prime} \leq k, u^{\prime} \neq u} \Phi_{p^{u^{\prime}}}(t) p^{l-k} & \text { if } u \leq k \\
p^{-l} \cdot t \cdot \frac{\mathrm{d}}{\mathrm{d} t} \Phi_{p^{u}}(t)\left(t^{p^{k}}-1\right) \prod_{k+1 \leq u^{\prime} \leq l, u^{\prime} \neq u} \Phi_{p^{u^{\prime}}}(t) & \text { if } u>k\end{cases} \\
& = \begin{cases}p^{-k} \cdot t \cdot \frac{\mathrm{d}}{\mathrm{d} t} \Phi_{p^{u}}(t) \prod_{0 \leq u^{\prime} \leq k, u^{\prime} \neq u} \Phi_{p^{u^{\prime}}}(t) & \text { if } u \leq k \\
0 & \text { if } u>k\end{cases}
\end{aligned}
$$

This shows the lemma. 
Proposition 22.8. Let $n$ be an integer. Then there is an isomorphism of rings

$$
\mathbb{Z}\left[n^{-1}\right][t] /\left\langle t^{n}-1\right\rangle \cong \bigoplus_{m \mid n} \mathbb{Z}\left[n^{-1}, \theta_{m}\right] .
$$

In particular, $\mathbb{Z}\left[n^{-1}\right][t] /\left\langle t^{n}-1\right\rangle$ is direct sum of Dedekind domains.

Proof. Define a ring homomorphism

$$
\iota_{m}: \mathbb{Z}\left[n^{-1}\right][t] /\left\langle\Phi_{m}\right\rangle \rightarrow \mathbb{Z}\left[n^{-1}\right][t] /\left\langle t^{n}-1\right\rangle, \quad[p(t)] \mapsto\left[p(t) \cdot \psi_{n, m}(t)\right] .
$$

This is well-defined since $\Phi_{m}(t) \cdot \psi_{n, m}(t)=0$ in $\mathbb{Z}\left[n^{-1}\right][t] /\left\langle t^{n}-1\right\rangle$. Let $\pi_{m}: \mathbb{Z}\left[n^{-1}\right][t] /\left\langle t^{n}-\right.$ $1\rangle \rightarrow \mathbb{Z}\left[n^{-1}\right][t] /\left\langle\Phi_{m}\right\rangle$ be the quotient map. Since $\sum_{m \mid n} \psi_{n, m}(t)=1$ and $\pi_{m}\left(\psi_{n, m^{\prime}}(t)\right)=0$ for $m \neq m^{\prime}$, we have $\pi_{m}\left(\psi_{n, m}(t)\right)=1$. Therefore, $\pi_{m} \circ \iota_{m}=\mathrm{id}$. $\Phi_{m} \mid \psi_{n, k}$ for $k \neq m$ implies $\pi_{k} \circ \iota_{m}=\delta_{k, m}$ id. $\sum_{m \mid n} \psi_{n, m}(t)=1$ also implies $\sum_{m \mid n} \iota_{m} \circ \pi_{m}=\mathrm{id}$. This shows

$$
\mathbb{Z}\left[n^{-1}\right][t] /\left\langle t^{n}-1\right\rangle \cong \bigoplus_{m \mid n} \mathbb{Z}\left[n^{-1}\right][t] /\left\langle\Phi_{m}\right\rangle .
$$

By Lemma 22.3 , there is an isomorphism $\mathbb{Z}\left[\theta_{m}\right] \cong \mathbb{Z}[t] /\left\langle\Phi_{m}\right\rangle$, which shows the first claim.

By $[11]$ Theorem $21.13, \mathbb{Z}\left[\theta_{m}\right]$ is equal to the algebraic integers of the algebraic number field $\mathbb{Q}\left[\theta_{m}\right]$ and hence a Dedekind domain. By [20], Theorem 29.6, an integral domain is Dedekind if and only if every non-zero ideal is invertible. Now let $S \subseteq D$ be multiplicative subset of a Dedekind domain $D$ and $I$ an ideal in $S^{-1} D$. Then $I \cap D$ is a non-zero ideal of $D$ and hence there is a fractional ideal $J$ of $D$ such that $I \cdot J=D$. Let $S^{-1} J$ be the fractional ideal of $S^{-1} D$, which is generated by $J$. It is easy to show that $S^{-1} J$ is an inverse of $I$. This shows that localizations of Dedekind domains are again Dedekind. In particular, $\mathbb{Z}\left[n^{-1}\right]\left[\theta_{m}\right]$ is a Dedekind domain

Lemma 22.9. Let $m, n \in \mathbb{Z}$ be coprime. Then there is an isomorphism of rings

$$
\mathbb{Z}\left[\theta_{n}\right] \otimes_{\mathbb{Z}} \mathbb{Z}\left[\theta_{m}\right] \cong \mathbb{Z}\left[\theta_{m n}\right]
$$

Proof. Claim: Let $m, n \in \mathbb{Z}$ be coprime, then $\Phi_{m}\left(t^{n}\right)=\prod_{d \mid n} \Phi_{m d}(t)$. We will prove the statement by induction over $m$. The case $m=1$ follows since $\Phi_{1}(t)=t-1$ and $t^{n}-1=\prod_{d \mid n} \Phi_{d}(t)$ by Theorem 22.2. For the induction step, let us assume that the statement is true for all $m^{\prime}<m$. Then

$$
\begin{aligned}
\Phi_{m}\left(t^{n}\right) \prod_{m^{\prime} \mid m, m^{\prime}<m} \Phi_{m^{\prime}}\left(t^{n}\right) & =t^{m n}-1=\prod_{d \mid m n} \Phi_{d}(t)=\prod_{m^{\prime} \mid m} \prod_{n^{\prime} \mid n} \Phi_{m^{\prime} n^{\prime}}(t) \\
& =\prod_{n^{\prime} \mid n} \Phi_{m n^{\prime}}(t) \prod_{m^{\prime} \mid m, m^{\prime}<m} \Phi_{m^{\prime}}\left(t^{n}\right),
\end{aligned}
$$

where the last equality uses the induction hypothesis. Now the claim follows since $\mathbb{Z}[t]$ is a unique factorization domain.

The isomorphism $\mathbb{Z}\left[\theta_{n}\right] \cong \mathbb{Z}[t] /\left\langle\Phi_{n}\right\rangle$ and the fact that $\Phi_{m n}(t)$ divides $\Phi_{m}\left(t^{n}\right)$ shows that $f_{m, n}: \mathbb{Z}\left[\theta_{m}\right] \rightarrow \mathbb{Z}\left[\theta_{m n}\right], \theta_{m} \mapsto \theta_{m}^{n}$ is a well-defined ring homomorphism. Let 
Appendix

$\mu_{n}: \mathbb{Z}\left[\theta_{n}\right] \otimes_{\mathbb{Z}} \mathbb{Z}\left[\theta_{n}\right] \rightarrow \mathbb{Z}\left[\theta_{n}\right]$ be the ring homomorphism which is given by multiplication. Define $F_{m, n}: \mathbb{Z}\left[\theta_{n}\right] \otimes_{\mathbb{Z}} \mathbb{Z}\left[\theta_{m}\right] \rightarrow \mathbb{Z}\left[\theta_{m n}\right]$ as the composition $F_{m, n}:=\mu_{m n} \circ\left(f_{n, m} \otimes_{\mathbb{Z}} f_{m, n}\right)$. Since $m$ and $n$ are coprime, there are $a, b \in \mathbb{Z}$ such that $a n+b m=1$. Therefore, $F_{m, n}\left(\theta_{n}^{b} \otimes_{\mathbb{Z}} \theta_{m}^{a}\right)=\theta_{m n}$, which shows that $F_{m, n}$ is surjective. Recall from Lemma 22.2 that $\phi$ denotes Euler's totient function. Since

$$
\operatorname{dim}_{\mathbb{Z}} \mathbb{Z}\left[\theta_{m n}\right]=\phi(m n)=\phi(n) \phi(m)=\operatorname{dim}_{\mathbb{Z}} \mathbb{Z}\left[\theta_{n}\right] \otimes_{\mathbb{Z}} \mathbb{Z}\left[\theta_{m}\right]
$$

$F_{m, n}$ is an isomorphism.

Lemma 22.10. Let $n, j \in \mathbb{N}$ and $p$ a prime number. Then

$$
\sum_{i=0}^{p^{n}-1} \theta_{p^{n}}^{i j}=\left\{\begin{array}{ll}
p^{n} & \text { if } p^{n} \mid j \\
0 & \text { otherwise }
\end{array} \text { in } \mathbb{Z}\left[\theta_{p^{n}}\right]\right.
$$

Proof. If $p^{n} \mid j$, then the statement is clear. Hence let us assume that $p^{n}$ does not divide $j$. Since $\theta_{p^{n}}^{i\left(j^{\prime}+r p^{n}\right)}=\theta_{p^{n}}^{i j^{\prime}}$ we may assume that $0<j<p^{n}$. There are $s, m \in \mathbb{N}$ with $0 \leq m<n, s \neq 0$ and $p \nmid s$ such that $j=p^{m} \cdot s$. Then

$$
\sum_{i=0}^{p^{n}-1} \theta_{p^{n}}^{i j}=\sum_{i=0}^{p^{n}-1} \theta_{p^{n-m}}^{i s}=p^{m} \sum_{i=0}^{p^{n-m}-1}\left(\theta_{p^{n-m}}^{s}\right)^{i}=0
$$

since $\theta_{p^{n-m}}^{s}$ is a $p^{n-m}$ th primitive root of unity.

Proposition 22.11. Let $m, n \in \mathbb{N}, n \leq m$ and $p$ a prime number. Then there is an isomorphism of rings

$$
\mathbb{Z}\left[p^{-1}, \theta_{p^{n}}\right] \otimes_{\mathbb{Z}} \mathbb{Z}\left[p^{-1}, \theta_{p^{m}}\right] \cong \underset{0<k<p^{n}, g c t(p, k)=1}{\bigoplus} \mathbb{Z}\left[p^{-1}, \theta_{p^{m}}\right]
$$

Proof. For $0<k<p^{n}, p \nmid\langle k$, define

$$
q_{k}:=\frac{1}{p^{n}} \sum_{j=0}^{p^{n}-1} \theta_{p^{n}}^{k j} \otimes \theta_{p^{m}}^{p^{m-n} j}
$$

Note that by Lemma 22.10, we have

$$
\sum_{0<k<p^{n}, \operatorname{gct}(p, k)=1} \theta_{p^{n}}^{j k}=-\sum_{i=0}^{p^{n-1}-1}\left(\theta_{p^{n}}^{p}\right)^{j i}
$$


for $0<j<p^{n}$. Hence we may compute

$$
\begin{aligned}
\sum_{0<k<p^{n}, \operatorname{gct}(p, k)=1} q_{k} & =\frac{1}{p^{n}} \sum_{j=0}^{p^{n}-1}\left(\sum_{0<k<p^{n}, \mathrm{gct}(p, k)=1} \theta_{p^{n}}^{j k}\right) \otimes \theta_{p^{m}}^{p^{m-n} j} \\
& =\frac{1}{p^{n}}\left(p^{n}-p^{n-1}-\sum_{j=1}^{p^{n}-1}\left(\sum_{i=0}^{p^{n-1}-1}\left(\theta_{p^{n}}^{p}\right)^{j i}\right) \otimes \theta_{p^{m}}^{p^{m-n} j}\right) \\
& =\frac{1}{p^{n}}\left(p^{n}-p^{n-1}-p^{n-1} 1 \otimes \sum_{j=1}^{p-1} \theta_{p^{m}}^{p^{m-1} j}\right) \\
& =\frac{1}{p^{n}}\left(p^{n}-p^{n-1}+p^{n-1}\right)=1 .
\end{aligned}
$$

Again by lemma 22.10 , we have the following identity in $\mathbb{Z}\left[\theta_{p^{n}}\right]$ for $0 \leq k, l<p^{n}$ and $j \in \mathbb{N}$ :

$$
\sum_{i=0}^{p^{n}-1} \theta_{p^{n}}^{k i+l(j-i)}=\theta_{p^{n}}^{l j} \sum_{i=0}^{p^{n}-1} \theta_{p^{n}}^{i(k-l)}=p^{n} \theta_{p^{n}}^{l j} \delta_{k, l} .
$$

Therefore

$$
q_{k} \cdot q_{l}=\frac{1}{p^{2 n}} \sum_{j=0}^{p^{n}-1}\left(\sum_{i=0}^{p^{n}-1} \theta_{p^{n}}^{k i+l(j-i)}\right) \otimes \theta_{p^{m}}^{p^{n-m} j}=\delta_{k, l} q_{k} .
$$

This shows that the $q_{k}$ for $0<k<p^{n}, \operatorname{gct}(p, k)=1$ are orthogonal idempotents in $\mathbb{Z}\left[p^{-1}, \theta_{p^{n}}\right] \otimes_{\mathbb{Z}} \mathbb{Z}\left[p^{-1}, \theta_{p^{m}}\right]$ that sum up to 1 .

For $0<k<p^{n}, \operatorname{gct}(p, k)=1$, define

$$
\iota_{k}: \mathbb{Z}\left[p^{-1}, \theta_{p^{m}}\right] \rightarrow \mathbb{Z}\left[p^{-1}, \theta_{p^{n}}\right] \otimes \mathbb{Z}\left[p^{-1}, \theta_{p^{m}}\right], \quad \theta_{p^{m}} \mapsto 1 \otimes \theta_{p^{m}} \cdot q_{k} .
$$

Furthermore, for every $0<k<p^{n}, \operatorname{gct}(p, k)=1$, let $a(k) \in \mathbb{N}$ such that $[a(k)]$ is the multiplicative inverse of $k$ in $\mathbb{Z} / p^{n}$. Define

$$
e_{a(k)}: \mathbb{Z}\left[p^{-1}, \theta_{p^{n}}\right] \rightarrow \mathbb{Z}\left[p^{-1}, \theta_{p^{n}}\right], \quad \theta_{p^{n}} \mapsto \theta_{p^{n}}^{-a(k)}
$$

and

$$
f_{p^{n}, p^{m}}: \mathbb{Z}\left[p^{-1}, \theta_{p^{n}}\right] \rightarrow \mathbb{Z}\left[p^{-1}, \theta_{p^{m}}\right], \quad \theta_{p^{n}} \mapsto \theta_{p^{m}}^{p^{m-n}} .
$$

Finally, let $\mu_{p^{m}}: \mathbb{Z}\left[p^{-1}, \theta_{p^{m}}\right] \otimes \mathbb{Z}\left[p^{-1}, \theta_{p^{m}}\right] \rightarrow \mathbb{Z}\left[p^{-1}, \theta_{p^{m}}\right]$ be the ring homomorphism which is induced by multiplication and set

$$
\pi_{k}:=\mu_{p^{m}} \circ\left(\left(f_{p^{n}, p^{m}} \circ e_{a(k)}\right) \otimes \operatorname{id}_{\mathbb{Z}\left[p^{-1}, \theta_{p^{m}}\right]}\right): \mathbb{Z}\left[p^{-1}, \theta_{p^{n}}\right] \otimes_{\mathbb{Z}} \mathbb{Z}\left[p^{-1}, \theta_{p^{m}}\right] \rightarrow \mathbb{Z}\left[p^{-1}, \theta_{p^{m}}\right] .
$$

For $0<k, l<p^{n}, p \nmid \nmid k$, we have

$$
\pi_{k}\left(q_{l}\right)=\frac{1}{p^{n}} \sum_{j=0}^{p^{n}-1}\left(\theta_{p^{m}}^{p^{m-n}}\right)^{(1-a(k) l) j}=\delta_{k, l} .
$$


Appendix

Therefore, $\pi_{l} \circ \iota_{k}=\delta_{k, l} \mathrm{id}_{\mathbb{Z}\left[p^{-1}, \theta_{p} m\right]}$. This shows that

$$
\bigoplus_{0<k<p^{n}, \operatorname{gct}(p, k)=1} \pi_{k}: \mathbb{Z}\left[p^{-1}, \theta_{p^{n}}\right] \otimes \mathbb{Z}\left[p^{-1}, \theta_{p^{m}}\right] \rightarrow \bigoplus_{0<k<p^{n}, \operatorname{gct}(p, k)=1} \mathbb{Z}\left[p^{-1}, \theta_{p^{m}}\right]
$$

is surjective. Since $\mathbb{Z}\left[p^{-1}\right]$ is a principal ideal domain and $\mathbb{Z}\left[p^{-1}, \theta_{p^{m}}\right]$ is a free $\mathbb{Z}\left[p^{-1}\right]$-module, the equality

$$
\begin{aligned}
\operatorname{dim}_{\mathbb{Z}\left[p^{-1}\right]}\left(\bigoplus_{0<k<p^{n}, \operatorname{gct}(p, k)=1} \mathbb{Z}\left[p^{-1}, \theta_{p^{m}}\right]\right) & =p^{n-1}(p-1) \operatorname{dim}_{\mathbb{Z}\left[p^{-1}\right]}\left(\mathbb{Z}\left[p^{-1}, \theta_{p^{m}}\right]\right) \\
& =\operatorname{dim}_{\mathbb{Z}\left[p^{-1}\right]}\left(\mathbb{Z}\left[p^{-1}, \theta_{p^{n}}\right] \otimes \mathbb{Z}\left[p^{-1}, \theta_{p^{m}}\right]\right)
\end{aligned}
$$

shows that $\bigoplus_{0<k<p^{n}, \operatorname{gct}(p, k)=1} \pi_{k}$ is an isomorphism.

\section{Miscellaneous Results}

Proposition 23.1. $G \ltimes\left[X^{H}\right] \cdot\left[X^{H^{\perp}}\right]=T_{\mathcal{C}(G)^{H}} \in \mathrm{KK}^{G}\left(G \ltimes G \ltimes \mathcal{C}(G)^{H}, \mathcal{C}(G)^{H}\right)$

Proof. Let us first describe $X^{H}$. We are using the description of a imprimitivity bimodule implementing the Morita-Rieffel equivalence between $G \ltimes \mathcal{C}(G)^{H}=G \ltimes \operatorname{Ind}_{H}^{G} \mathbb{C}$ and $C^{*} H$ given in [48], Theorem 4.22 composed with the isomorphism $\mathcal{F}_{H^{\perp}}^{-1}: C^{*} H \cong \mathcal{C}(G)^{H^{\perp}}$ from the right. As a vector space, $X^{H}$ is given by $\mathcal{C}(G)$, the left and right Hilbert module structure is given by the following formulas: Let $x, y \in X^{H}={ }_{v s} \mathcal{C}(G), e \in G \ltimes \mathcal{C}(G)^{H}={ }_{v s}$ $\mathcal{C}(G \times G)^{\{0\} \times H}$ and $b \in \mathcal{C}(G)^{H^{\perp}}$, then

$$
\begin{aligned}
e \cdot x(r) & =\int_{G} e(t, r) x(r-t) \mathrm{d} t ; \\
x \cdot b(r) & =\frac{1}{|G|} \int_{G} \int_{G} x(r-t) \chi(t, s) b(s) \mathrm{d} s \mathrm{~d} t ; \\
G \ltimes \mathcal{C}(G)^{H}\langle x, y\rangle(t, r) & =\int_{H} x(r+s) \overline{y(r+s-t)} \mathrm{d} s ; \\
\langle x, y\rangle_{\mathcal{C}(G)^{H^{\perp}}}(r) & =\int_{G} \int_{H} \chi(r, s)^{-1} \overline{x(-t)} y(s-t) \mathrm{d} t \mathrm{~d} s .
\end{aligned}
$$

The action of $G$ on $X^{H}$ is given by $\gamma_{l} x(s)=\chi(s, l) x(s)$. Recall that by definition $T_{A}=\left[\Phi_{A}\right] \cdot E_{A}$. The inverse of $E_{A}$ as a KK-element is given by the class of the equivariant *-homomorphism

$$
\mu_{A}: A \rightarrow \mathbb{K}\left(L^{2}(G, A)\right), \quad \mu_{A}(a)(f)(s)=a \frac{1}{|G|} \int_{G} f(t) \mathrm{d} t .
$$

Hence it is sufficient to show that $\left(\Phi_{\mathcal{C}(G)^{H}}^{-1} \circ \mu_{\mathcal{C}(G)^{H}}\right)^{*} G \ltimes X^{H}$ is the dual Morita-Rieffel imprimitivity bimodule of $X^{H^{\perp}}$. First let us check that

$$
\Phi_{\mathcal{C}(G)^{H}}^{-1} \circ \mu_{\mathcal{C}(G)^{H}}: \mathcal{C}(G)^{H} \rightarrow G \ltimes G \ltimes \mathcal{C}(G)^{H}
$$


is equal to

$$
\begin{aligned}
& \iota: \mathcal{C}(G)^{H} \rightarrow G \ltimes G \ltimes \mathcal{C}(G)^{H}, \quad \iota(F)(t, s)=\chi(t, s) \frac{1}{|G|^{2}} \int_{G} \lambda_{w}(F) \overline{\chi(t, w)} \mathrm{d} w: \\
& \Phi_{\mathcal{C}(G)^{H}}(\iota(F))(f)(r)=\int_{G} \int_{G} \lambda_{-r}(\iota(F)(t, s)) \chi(t, r-s) f(r-s) \mathrm{d} t \mathrm{~d} s \\
& =\frac{1}{|G|^{2}} \int_{G} \int_{G} \int_{G} \lambda_{w-r}(F) \chi(t, r-w) f(r-s) \mathrm{d} w \mathrm{~d} t \mathrm{~d} s \\
& =\frac{1}{|G|} \int_{G} \int_{G} \lambda_{w-r}(F) \delta_{0}(r-w) f(r-s) \mathrm{d} w \mathrm{~d} s \\
& =F \frac{1}{|G|} \int_{G} f(r-s) \mathrm{d} s \\
& =\mu_{\mathcal{C}(G)^{H}}(F)(f)(r) \text {. }
\end{aligned}
$$

This shows $\Phi_{\mathcal{C}(G)^{H}} \circ \iota=\mu_{\mathcal{C}(G)^{H}}$. Therefore, $\Phi_{\mathcal{C}(G)^{H}}^{-1} \circ \mu_{\mathcal{C}(G)^{H}}=\iota$.

$\iota^{*}\left(G \ltimes X^{H}\right)$ as a KK-element is equal to the Kasparov $\mathcal{C}(G)^{H}-G \ltimes \mathcal{C}(G)^{H^{\perp}}$-module $\iota\left(1_{\mathcal{C}(G)^{H}}\right) \cdot G \ltimes X^{H}$ with the zero operator. $G \ltimes X^{H}$ as a vector space is given by $\mathcal{C}(G \times G)$. Let $\xi \in G \ltimes X^{H}$ and $F \in \mathcal{C}(G)^{H}$ then

$$
\begin{aligned}
(\iota(F) \cdot \xi)(v, u) & =\int_{G}\left(\iota(F)(t) \cdot \gamma_{t}(\xi(v-t))\right)(u) \mathrm{d} t \\
& =\int_{G} \int_{G} \iota(F)(t, s, u) \gamma_{t}(\xi(v-t))(u-s) \mathrm{d} s d t \\
& =\frac{1}{|G|^{2}} \int_{G} \int_{G} \int_{G} \chi(t, s) F(u-w) \overline{\chi(t, w)} \chi(t, u-s) \xi(v-t, s-u) \mathrm{d} w \mathrm{~d} s \mathrm{~d} t \\
& =\frac{1}{|G|^{2}} \int_{G} \int_{G} \int_{G} F(u-w) \chi(t, u-w) \xi(v-t, s-u) \mathrm{d} w \mathrm{~d} s \mathrm{~d} t \\
& =\frac{1}{|G|^{2}} \int_{G} \int_{G} \int_{G} F(w) \chi(v-t, w) \xi(t, s) \mathrm{d} w \mathrm{~d} s \mathrm{~d} t \\
& =\int_{G} \mathcal{F}(F)(v-t)\left(\int_{G} \frac{1}{|G|} \xi(t, s) \mathrm{d} s\right) \mathrm{d} t .
\end{aligned}
$$

In particular

$$
\left(\iota\left(1_{\mathcal{C}(G)^{H}}\right) \cdot \xi\right)(v, u)=\frac{1}{|G|} \int_{G} \xi(v, s) \mathrm{d} s .
$$

This shows that as a vector space, $\iota\left(1_{\mathcal{C}(G)^{H}}\right) \cdot G \ltimes X^{H}$ is isomorphic to $\mathcal{C}(G)$ via

$$
j: \mathcal{C}(G) \rightarrow \iota\left(1_{\mathcal{C}(G)^{H}}\right) \cdot G \ltimes X^{H}, j(f)(t, s)=f(t) .
$$

Recall from [37] the definition of the dual Morita-Rieffel imprimitivity bimodule: Let $Y^{H}$ denote the dual of $X^{H^{\perp}}$. Then there is a antilinear bijection $\beta: \mathcal{C}(G) \rightarrow Y^{H}$. Left 
Appendix

and right multiplication and the $G \ltimes \mathcal{C}(G)^{H^{\perp}}$-valued inner product are given by the formulas

$$
\begin{aligned}
\beta^{-1}(b \cdot \beta(x))(r) & =x \cdot b^{*}(r)=\frac{1}{|G|} \int_{G} \int_{G} x(r-t) \chi(t, s) \overline{b(s)} \mathrm{d} s \mathrm{~d} t ; \\
\beta^{-1}(\beta(x) \cdot e)(r) & =e^{*} \cdot x(r)=\int_{G} \overline{e(-t, r-t)} x(r-t) \mathrm{d} t ; \\
\langle\beta(x), \beta(y)\rangle_{G \ltimes \mathcal{C}(G)^{H^{\perp}}}(t, r) & =\langle x, y\rangle=\int_{H} x(r+s) \overline{y(r+s-t)} \mathrm{d} s,
\end{aligned}
$$

for $b \in \mathcal{C}(G)^{H}, e \in G \ltimes \mathcal{C}(G)^{H^{\perp}}, x, y \in X^{H}=\mathcal{C}(G)$

Claim:

$$
\eta_{H}: Y^{H} \rightarrow \iota\left(1_{\mathcal{C}(G)^{H}}\right) \cdot G \ltimes X^{H}, \quad \eta(\beta(f))(t, s)=\frac{\overline{f(-t)}}{\sqrt{\left(|G|\left|H^{\perp}\right|\right)}}
$$

gives an isomorphism of Kasparov $\mathcal{C}(G)^{H}-G \ltimes \mathcal{C}(G)^{H^{\perp}}$-modules. It is clear that $\eta$ is a bijection, hence we only have to show that it intertwines left and right multiplication and the inner product. Let us abbreviate $\frac{1}{\sqrt{\left(|G|\left|H^{\perp}\right|\right)}}$ by $c_{H}$.

$$
\begin{aligned}
\eta(b \cdot \beta(x))(r, u) & =\eta\left(\beta\left(x \cdot b^{*}\right)\right)(r, u)=\frac{c_{H}}{|G|} \int_{G} \int_{G} \overline{x(-r-t)} \chi(-t, s) b(s) \mathrm{d} s \mathrm{~d} t \\
& =c_{H} \int_{G} \overline{x(t-r)} \mathcal{F}(b)(t) \mathrm{d} t=c_{H} \int_{G} \mathcal{F}(b)(r-t) \overline{x(-t)} \mathrm{d} t \\
& =\iota(b) \cdot \eta(\beta(x))(r, u) \\
\eta(\beta(x) \cdot e)(r, u) & =c_{H} \int_{G} e(-t,-r-t) \overline{x(-r-t)} \mathrm{d} t \\
& =c_{H} \int_{G} \overline{x(-l)} e(r-l,-l) \mathrm{d} l \\
& =\frac{c_{H}}{|G|} \int_{G} \int_{G} \int_{G} \overline{x(-l)} \chi(t, s) e(r-l, s-l) \mathrm{d} l \mathrm{~d} s \mathrm{~d} t \\
& =\frac{1}{|G|} \int_{G} \int_{G} \int_{G} \eta(\beta(x))(l, u-t) \chi(t, s) e(r-l, s-l) \mathrm{d} l \mathrm{~d} s \mathrm{~d} t \\
& =\int_{G}\left(\eta(\beta(x))(l) \cdot \lambda_{l}(e(r-l))\right)(u) \mathrm{d} l \\
& =\eta(\beta(x)) \cdot e(r, u) .
\end{aligned}
$$




$$
\begin{aligned}
\langle\eta(\beta(x)), \eta(\beta(y))\rangle(t, r) & =\int_{G}(\langle\eta(\beta(x))(l), \eta(\beta(y))(t+l)\rangle)(r+l) \mathrm{d} l \\
& =\int_{G} \int_{G} \int_{H} \chi(r+l, v)^{-1} \overline{\eta(\beta(x))(l,-u)} \eta(\beta(y))(t+l, v-u) \mathrm{d} l \mathrm{~d} u \mathrm{~d} v \\
& =c_{H}^{2} \int_{G} \int_{G} \int_{H} \chi(r+l,-v) x(-l) \overline{y(-t-l)} \mathrm{d} l \mathrm{~d} u \mathrm{~d} v \\
& =c_{H}^{2}|G| \int_{G} \int_{H} \chi(r+l,-v) x(-l) \overline{y(-t-l)} \mathrm{d} l \mathrm{~d} v \\
& =c_{H}^{2}|G||H| \int_{G} 1_{H^{\perp}}(r+l) x(-l) \overline{y(-t-l)} \mathrm{d} l \\
& =\int_{G} 1_{H^{\perp}}(l) x(r-l) \overline{y(r-t-l)} \mathrm{d} l \\
& =\int_{H^{\perp}} x(r+s) \overline{y(r+s-t)} \mathrm{d} s \\
& =\langle\beta(x), \beta(y)\rangle(t, r) .
\end{aligned}
$$

This shows that $\iota^{*}\left(G \ltimes X^{H}\right)$ is isomorphic to $Y^{H}$-the dual of $X^{H^{\perp}}$-as a Kasparov $\mathcal{C}(G)^{H}-G \ltimes \mathcal{C}(G)^{H^{\perp}}$-module.

Lemma 23.6. Let $R=\mathbb{Z}[t, s] /\langle(t-1)(s-1), N(t)+N(s)-p\rangle, c \in \mathbb{N}$ and define $N_{c}$ to be the free abelian group on generators $\left\{q_{0}, \ldots, q_{p-2}, r_{0}, \ldots r_{p-2}\right\}$. Define $s$ and $t$ on the $\mathbb{Z}$-basis $\left\{q_{0}, \ldots, q_{p-2}, r_{0}, \ldots r_{p-2}\right\}$ via

1. $t \cdot q_{i}:=q_{i}+c r_{0}$,

2. $t \cdot r_{i}:=r_{i+1}$ for $i<p-2$ and $t \cdot r_{p-2}:=-\sum_{k=0}^{p-2} r_{k}$,

3. $s \cdot q_{i}:=q_{i+1}$ for $i<p-2$ and $s \cdot q_{p-2}:=-c \sum_{k=0}^{p-2}(p-1-k) r_{k}-\sum_{k=0}^{p-2} q_{k}$,

4. $s \cdot r_{k}=r_{k}$.

Then $N_{c}$ is a well-defined $R$-module.

Proof. Let us first check that $t s=s t$ on $N_{c}$ : We have $t s q_{i}=t q_{i+1}=q_{i+1}+c r_{0}=s t q_{i}$ for $0 \leq i<p-2$ and

$$
\begin{aligned}
t s q_{p-2} & =t\left(-c \sum_{k=0}^{p-2}(p-1-k) r_{k}-\sum_{k=0}^{p-2} q_{k}\right) \\
& =-c \sum_{k=1}^{p-2}(p-k) r_{k}+c \sum_{k=0}^{p-2} r_{k}-\sum_{k=0}^{p-2} q_{k}-(p-1) c r_{0} \\
& =-c \sum_{k=0}^{p-2}(p-1-k) r_{k}-\sum_{k=0}^{p-2} q_{k}+c r_{0} \\
& =s\left(q_{p-2}+c r_{0}\right)=s t q_{p-2} .
\end{aligned}
$$




\section{Appendix}

Furthermore, we have $s t r_{i}=s r_{i+1}=r_{i+1}=t s r_{i+1}$ for $0 \leq i<p-2$ and $s t r_{p-2}=$ $s\left(-\sum_{k=0}^{p-2} r_{k}\right)=-\sum_{k=0}^{p-2} r_{k}=t s r_{p-2}$. This shows that $N_{c}$ is a $\mathbb{Z}[t, s]$-module. The next step is to verify that the relations $(t-1)(s-1)=0$ and $N(t)+N(s)=p$ hold. We have $(s t+1) q_{i}=q_{i+1}+c r_{0}+q_{i}=(s+t) q_{i}$ for $0 \leq i<p-2$ and

$$
(s t+1) q_{p-2}=-c \sum_{k=0}^{p-2}(p-1-k) r_{k}-\sum_{k=0}^{p-2} q_{k}+c r_{0}+q_{p-2}=(s+t) q_{p-2} .
$$

Similarly, we compute $(s t+1) r_{i}=r_{i+1}+r_{i}=(s+t) r_{i}$ and $(s t+1) r_{p-2}=-\sum_{k=0}^{p-2} r_{k}+$ $r_{p-2}=(s+t) r_{p-2}$. Therefore, we are left with the verification of $N(t)+N(s)=p$. First compute

$$
\begin{gathered}
s^{2} q_{p-2}=s\left(-c \sum_{k=0}^{p-2}(p-1-k) r_{k}-\sum_{k=0}^{p-2} q_{k}\right)=-c \sum_{k=0}^{p-2}(p-1-k) r_{k}-\sum_{k=0}^{p-2} s q_{k} \\
=-\sum_{k=1}^{p-2} q_{k}+\sum_{k=0}^{p-2} q_{k}=q_{0} .
\end{gathered}
$$

This shows that

$$
N(s) q_{i}=N(s) q_{0}=\sum_{k=0}^{p-2} q_{k}-c \sum_{k=0}^{p-2}(p-1-k) r_{k}-\sum_{k=0}^{p-2} s q_{k}=-c \sum_{k=0}^{p-2}(p-1-k) r_{k}
$$

for all $i=0, \ldots p-2$. Furthermore we have

$$
t^{k} q_{i}=t^{k-1} q_{i}+c t^{k-1} r_{0}=t^{k-1} q_{i}+c r_{k-1}=\ldots=q_{i}+c \sum_{j=0}^{k-1} r_{j} .
$$

Therefore, $N(t) q_{i}=p q_{i}+c \sum_{k=0}^{p-2}(p-1-k) r_{k}$. This shows that $(N(t)+N(s)) q_{i}=p q_{i}$ for all $i=0, \ldots p-2$.

Since $t^{2} r_{p-2}=r_{0}$, we have $N(t) r_{i}=N(t) r_{0}=\sum_{k=0}^{p-2} r_{k}-\sum_{k=0}^{p-2} r_{k}=0$. On the other hand, $N(s) r_{i}=p r_{i}$. This shows that $(N(t)+N(s)) r_{i}=p r_{i}$ and finishes the proof. 


\section{Bibliography}

[1] S. Baaj and G. Skandalis. $C^{*}$-algèbres de Hopf et théorie de Kasparov équivariante. K-Theory, 2(6):683-721, 1989.

[2] P. Baum, A. Connes, and N. Higson. Classifying space for proper actions and Ktheory of group $C^{*}$-algebras. In $C^{*}$-algebras: 1943-1993 (San Antonio, TX, 1993), volume 167 of Contemp. Math., pages 240-291. Amer. Math. Soc., Providence, RI, 1994.

[3] A. Beligiannis. Relative homological algebra and purity in triangulated categories. J. Algebra, 227(1):268-361, 2000.

[4] R. Bentmann. Filtrated K-theory and classification of $C^{*}$-algebras. Master's thesis, Georg-August Universität Göttingen, 2010.

[5] R. Bentmann and M. Köhler. Universal coefficient theorems for $C^{*}$-algebras over finite topological spaces. to appear.

[6] B. Blackadar. K-theory for operator algebras, volume 5 of Mathematical Sciences Research Institute Publications. Cambridge University Press, Cambridge, second edition, 1998.

[7] N. P. Brown and N. Ozawa. $C^{*}$-algebras and finite-dimensional approximations, volume 88 of Graduate Studies in Mathematics. American Mathematical Society, Providence, RI, 2008.

[8] M. C. R. Butler, J. M. Campbell, and L. G. Kovács. On infinite rank integral representations of groups and orders of finite lattice type. Arch. Math. (Basel), 83(4):297-308, 2004.

[9] M. D. Choi and E. G. Effros. The completely positive lifting problem for $C^{*}$ algebras. Ann. of Math. (2), 104(3):585-609, 1976.

[10] J. D. Christensen. Ideals in triangulated categories: phantoms, ghosts and skeleta. Adv. Math., 136(2):284-339, 1998.

[11] C. W. Curtis and I. Reiner. Representation theory of finite groups and associative algebras. Pure and Applied Mathematics, Vol. XI. Interscience Publishers, a division of John Wiley \& Sons, New York-London, 1962.

[12] S. Echterhoff. The Green-Julg theorem, 2000. Available online at: http://wwwmath.uni-muenster.de/u/paravici/FocusedSemester/lecturenotes/green-julg-Echterhoff.pdf. 
Bibliography

[13] S. Eilenberg and J. C. Moore. Foundations of relative homological algebra. Mem. Amer. Math. Soc. No., 55:39, 1965.

[14] Elliot. On the classification of inductive limits of sequences of semisimple finitedimensional algebras. Journal of algebra, 38(1):29, 1976.

[15] U. Haag. On $\mathbf{Z} / 2 \mathbf{Z}$-graded KK-theory and its relation with the graded Ext-functor. J. Operator Theory, 42(1):3-36, 1999.

[16] B. Hartley and T. O. Hawkes. Rings, modules and linear algebra. Chapman \& Hall, London, 1980. A further course in algebra describing the structure of abelian groups and canonical forms of matrices through the study of rings and modules, A reprinting.

[17] N. Higson and G. Kasparov. E-theory and KK-theory for groups which act properly and isometrically on Hilbert space. Invent. Math., 144(1):23-74, 2001.

[18] J. A. Hillman. Polynomials determining dedekind domains. Bulletin of the Australian Mathematical Society, 29:167 -175, 1984.

[19] H. Inasaridze, T. Kandelaki, and R. Meyer. Localisation and colocalisation of KKtheory at sets of primes. available online at: http://arxiv.org/abs/1003.0278, March 2010 .

[20] I. M. Isaacs. Algebra: a graduate course, volume 100 of Graduate Studies in Mathematics. American Mathematical Society, Providence, RI, 2009. Reprint of the 1994 original.

[21] P. Julg. K-théorie équivariante et produits croisés. C. R. Acad. Sci. Paris Sér. I Math., 292(13):629-632, 1981.

[22] G. G. Kasparov. The operator K-functor and extensions of $C^{*}$-algebras. Math. USSR Izv., 16:513-572, 1981.

[23] G. G. Kasparov. Equivariant KK-theory and the Novikov conjecture. Invent. Math., 91(1):147-201, 1988.

[24] M. Maghfoul. Semi-exactitude du bifoncteur de Kasparov équivariant. K-Theory, 16(3):245-276, 1999.

[25] J. P. May. Equivariant homotopy and cohomology theory, volume 91 of CBMS Regional Conference Series in Mathematics. Published for the Conference Board of the Mathematical Sciences, Washington, DC, 1996. With contributions by M. Cole, G. Comezaña, S. Costenoble, A. D. Elmendorf, J. P. C. Greenlees, L. G. Lewis, Jr., R. J. Piacenza, G. Triantafillou, and S. Waner.

[26] R. Meyer. Categorical aspects of bivariant K-theory. In K-theory and noncommutative geometry, EMS Ser. Congr. Rep., pages 1-39. Eur. Math. Soc., Zürich, 2008 . 
[27] R. Meyer and R. Nest. The Baum-Connes conjecture via localisation of categories. Topology, 45(2):209-259, 2006.

[28] R. Meyer and R. Nest. C*-algebras over topological spaces: Filtrated K-theory, 2008. Preprint, available online at: http://arxiv.org/abs/0810.0096.

[29] R. Meyer and R. Nest. Homological algebra in bivariant K-theory and other triangulated categories, 2008. Preprint, available online at: http://arxiv.org/abs/math/0702146.

[30] R. Meyer and R. Nest. $C^{*}$-algebras over topological spaces: the bootstrap class. Münster J. Math., 2:215-252, 2009.

[31] A. Neeman. Triangulated categories, volume 148 of Annals of Mathematics Studies. Princeton University Press, Princeton, NJ, 2001.

[32] F. Peter and H. Weyl. Die Vollständigkeit der primitiven Darstellungen einer geschlossenen kontinuierlichen Gruppe. Math. Ann., 97(1):737-755, 1927.

[33] N. C. Phillips. Equivariant K-theory and freeness of group actions on $C^{*}$-algebras, volume 1274 of Lecture Notes in Mathematics. Springer-Verlag, Berlin, 1987.

[34] N. C. Phillips. A classification theorem for nuclear purely infinite simple $C^{*}-$ algebras. Doc. Math., 5:49-114 (electronic), 2000.

[35] G. Pisier. Similarity problems and completely bounded maps, volume 1618 of Lecture Notes in Mathematics. Springer-Verlag, Berlin, expanded edition, 2001. Includes the solution to "The Halmos problem".

[36] V. V. Prasolov. Polynomials, volume 11 of Algorithms and Computation in Mathematics. Springer-Verlag, Berlin, 2004. Translated from the 2001 Russian second edition by Dimitry Leites.

[37] I. Raeburn and D. P. Williams. Morita equivalence and continuous-trace $C^{*}$ algebras, volume 60 of Mathematical Surveys and Monographs. American Mathematical Society, Providence, RI, 1998.

[38] I. Reiner. Integral representations of cyclic groups of prime order. Proc. Amer. Math. Soc., 8:142-146, 1957.

[39] D. W. Roeder. Category theory applied to Pontryagin duality. Pacific J. Math., 52:519-527, 1974

[40] J. Rosenberg and C. Schochet. The Künneth theorem and the universal coefficient theorem for equivariant K-theory and KK-theory. Mem. Amer. Math. Soc., 62(348):vi+95, 1986.

[41] J. Rosenberg and C. Schochet. The Künneth theorem and the universal coefficient theorem for Kasparov's generalized K-functor. Duke Math. J., 55(2):431-474, 1987. 
Bibliography

[42] G. Skandalis. Une notion de nucléarité en K-théorie (d'après J. Cuntz). K-Theory, 1(6):549-573, 1988.

[43] H. Takai. On a duality for crossed products of $C^{*}$-algebras. J. Functional Analysis, 19:25-39, 1975.

[44] J. L. Taylor. Banach algebras and topology. In Algebras in analysis (Proc. Instructional Conf. and NATO Advanced Study Inst., Birmingham, 1973), pages 118-186. Academic Press, London, 1975.

[45] A. B. Thom. Connective E-theory and bivariant homology. PhD thesis, Univ. Münster, Mathematisch-Naturwissenschaftliche Fakultät, 2003.

[46] A. Van Daele. K-theory for graded Banach algebras. I. Quart. J. Math. Oxford Ser. (2), 39(154):185-199, 1988.

[47] C. A. Weibel. An introduction to homological algebra, volume 38 of Cambridge Studies in Advanced Mathematics. Cambridge University Press, Cambridge, 1994.

[48] D. P. Williams. Crossed products of $C^{*}$-algebras, volume 134 of Mathematical Surveys and Monographs. American Mathematical Society, Providence, RI, 2007. 